\title{
Quantitation of Fentanyl and Metabolites from Blow Fly Tissue and Development Effects of Fentanyl on Lucilia sericata
}

Joseph Allen Cox

West Virginia University, jc0128@mix.wvu.edu

Follow this and additional works at: https://researchrepository.wvu.edu/etd

Part of the Entomology Commons, and the Toxicology Commons

\section{Recommended Citation}

Cox, Joseph Allen, "Quantitation of Fentanyl and Metabolites from Blow Fly Tissue and Development Effects of Fentanyl on Lucilia sericata" (2021). Graduate Theses, Dissertations, and Problem Reports. 10276.

https://researchrepository.wvu.edu/etd/10276

This Dissertation is protected by copyright and/or related rights. It has been brought to you by the The Research Repository @ WVU with permission from the rights-holder(s). You are free to use this Dissertation in any way that is permitted by the copyright and related rights legislation that applies to your use. For other uses you must obtain permission from the rights-holder(s) directly, unless additional rights are indicated by a Creative Commons license in the record and/ or on the work itself. This Dissertation has been accepted for inclusion in WVU Graduate Theses, Dissertations, and Problem Reports collection by an authorized administrator of The Research Repository @ WVU.

For more information, please contact researchrepository@mail.wvu.edu. 


\title{
Quantitation of Fentanyl and Metabolites from Blow Fly Tissue and Development Effects of Fentanyl on Lucilia sericata
}

\author{
Joseph Cox
}

\begin{abstract}
Dissertation submitted to the Eberly College of Arts and Sciences at West Virginia University

in partial fulfillment of the requirements for the degree of
\end{abstract}

Doctor of Philosophy in

Forensic Science

Luis E. Arroyo, Ph.D., Chair

Rachel Mohr, Ph.D.

Tatiana Trejos, Ph.D.

Joseph DelTondo, DO

Department of Forensic and Investigative Science

Morgantown, West Virginia

2021

Keywords: Fentanyl, Lucilia sericata, QuEChERS, Forensic Toxicology, Postmortem Interval, LC-MS/MS

Copyright 2021 Joseph Cox 


\section{Abstract \\ Quantitation of Fentanyl and Metabolites from Blow Fly Tissue and Development Effects of Fentanyl on Lucilia sericata}

$$
\text { Joseph Cox }
$$

Death investigations often involve collaborative work between different forensic disciplines, especially with regards to pathology, toxicology, and entomology. In death cases consisting of individuals who have no apparent trauma, a routine postmortem toxicology analysis is performed. Here, coroners and medical examiners traditionally rely upon matrices such as blood, urine, gastric content and vitreous to provide toxicological interpretation of drug concentrations. However, in absence of these tissue samples in decedents with advanced decomposition or skeletonized, insects have proven resourceful in supporting valuable analytical information. In fact, beyond 72 hours, entomology evidence is considered the most reliable source to estimate the minimum postmortem interval ( $\min P M I)$. The $\min P M I$ is the minimum amount of time that it would take for an insect to reach a physical character such as length or life stage and, therefore, is the minimum time that an individual has been deceased.

The succession that occurs on a corpse after death is a relatively confined and closely packed ecosystem that is typically restricted to the remains and environment proximity. The incorporation of drugs into the insects is possible and open an opportunity for assessing drug presence and developmental effects in insect tissue. Various drugs accelerate insect growth, like codeine, cocaine, diazepam, and methamphetamine. In contrast, others like tramadol, methadone, methylphenidate, and morphine have been shown to slow the development of forensically relevant insects. The significance of these changes is the impact on the ${ }_{\min } \mathrm{PMI}$ calculation that can be incorrectly determined based on the insect's size or stage of growth if drugs are present when the insects are actively feeding.

This dissertation investigates the effects of designer opioids on the development of blow fly (Lucilia sericata) larvae and the ability to detect and quantify the drugs and/or metabolites present in each stage of the fly's life cycle. Therefore, we evaluated human tissue fortified with fentanyl at four treatment concentrations, and blow fly larvae were placed to feed on the drug infused tissue. A quick, easy, cheap, effective, rugged, and safe (QuEChERS) extraction method was developed, validated, and applied to extract fentanyl and metabolites from the larva, pupa, pupa casings, adults of Lucilia sericata. Additionally, since liver tissue was utilized as feeding media, a complete validation extraction was also developed, followed by the quantitative analysis of target drugs and metabolites via liquid-chromatography-tandem mass spectrometry (LC-MS/MS).

This research represents an advancement in the combat of the opioid epidemic by offering a fully validated protocol that complements the analytical toolkit in forensic toxicology laboratories and medical examiners offices for complex biological matrices by 1) providing a protocol to extract and quantify fentanyl and metabolites from complex matrices using QuEChERS to aid in forensic investigations, 2) an evaluation any growth effects fentanyl has on the development of the insects and the potential implications on the impact of ${ }_{\min } \mathrm{PMI}$ estimations, 3) conclusions on the ability to correlate the concentration of fentanyl and metabolites from the insect tissue to the initial concentration of the feeding media. 


\section{Funding Statement}

This study was funded by the National Institute of Justice, Award \#2019-R2-CX-037, NIJ- STEM Graduate Fellowship. The title of the NIJ fellowship was "Extraction and Quantification of Fentanyl and Metabolites from Complex Biological Matrices to Support Medicolegal Death Investigations" (Jan 2020Dec 2021). 


\section{Acknowledgements}

Through this adventure of earning my Ph.D, I am proud to say that I have learned from the best in the field of forensic science. Accomplishing this has been challenging and a great experience that I will cherish forever. I could not have reached the end of this mission without my great team to support me along the way and I thank y'all for being there for me.

Most importantly I would like to thank my mentor and committee chair, Dr. Luis Arroyo. He was always there for me when I needed support and provided countless opportunities to advance my professional and personal life. I will never be able to thank you enough and will be eternally grateful for my time at WVU.

I would also like to thank my other committee members; without your guidance I would not be at this point today. Dr. Rachel Mohr, your help and guidance in terms of forensic entomology will remain with me forever. You were always helpful when I needed you and your knowledge of entomology was vital to my project, thank you. Dr. Tatiana Trejos, without your help with my project design and statistical models I would not have been able to produce this project. You were always there when I need professional or personal guidance and I thank you for making my time at WVU memorable. Dr. Joseph DelTondo, your assistance with pathology and opening of doors for connections that I would not have obtain on my own was invaluable. You were always willing to assist in any way you could, and I appreciate your willingness to help. Dr. Suzanne Bell, though your time on my committee was limited, your guidance during my project proposal provided a large impact on my project and your guidance as a mentor was greatly appreciated. I would like to thank Dr. James Kraner for his support from the medical examiner's office and for his guidance.

I would also like to thank my research group, without y'all life would have been boring and dull. We always found ways to entertain ourselves while still accomplishing so much. I would like to specifically thank Colby Ott, Kylea Mathison (Morris), Kourtney Dalzell, Sara Kuberski, Travon Cooman, Bill Feeney, and Katherine Davis for their help in making this journey memorable. Colby, you are a great friend and an awesome roommate.

Finally, I would like to thank my family: my mother Ruth Cox, my grandmother Betty Cox, and my brother Brandon Cox, for their support during this time. I am sure they felt like this journey had no end, but the mountain has been climbed. During this climb, I was lucky enough to meet a colleague that I hope will be with me for a lifetime, Roberta Gorziza. You started as a visiting scholar and I am proud to call you my fiancé. Without you this journey would have been insurmountable, I am so lucky to have found you. I love you.

There are no such thing as applied sciences, only application of science. -Louis Pasteur 


\section{Table of Contents}

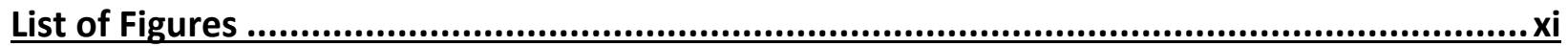

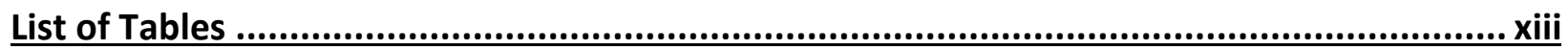

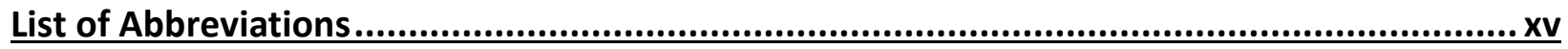

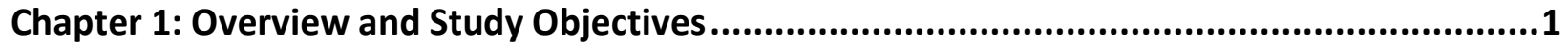

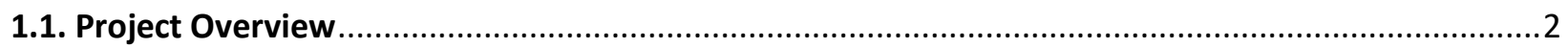

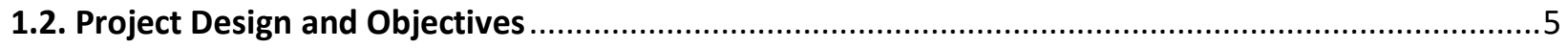

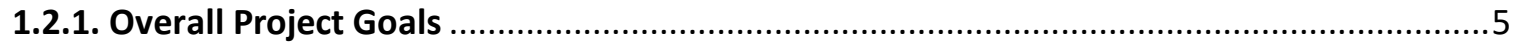

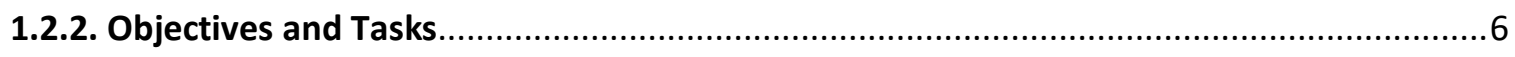

Chapter 2: Introduction and Literature Review......................................................... 10

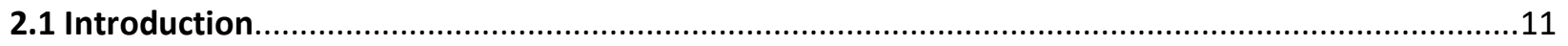

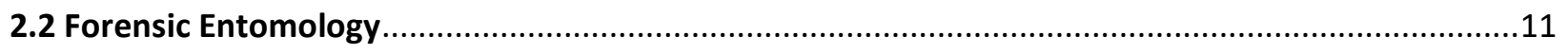

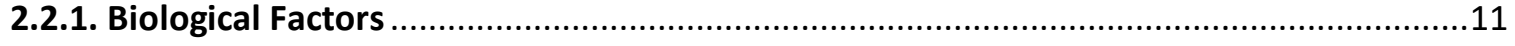

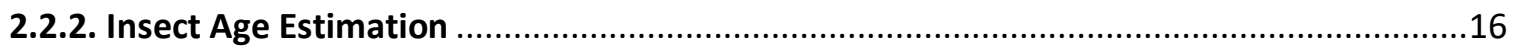

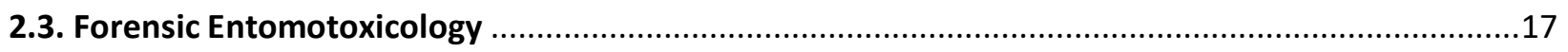

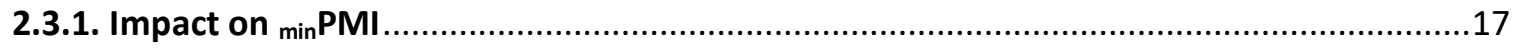

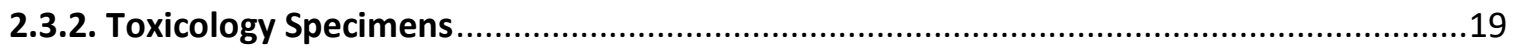

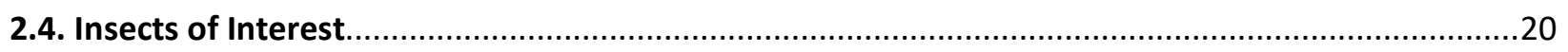

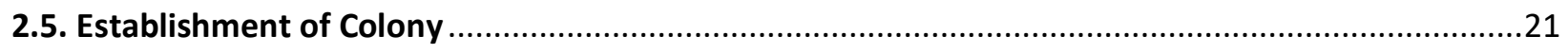

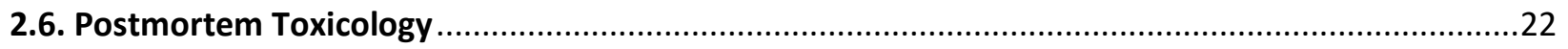

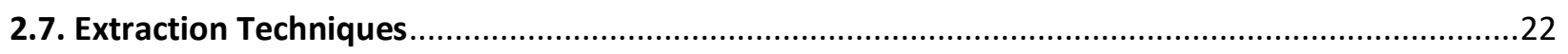

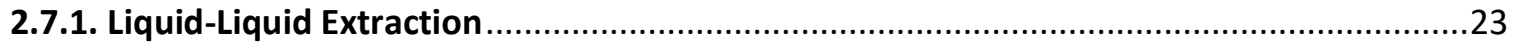

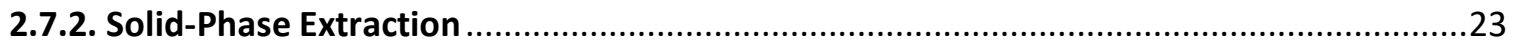

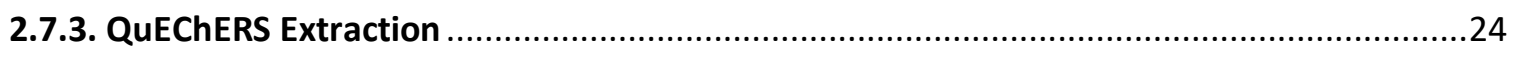

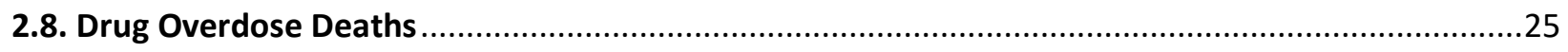

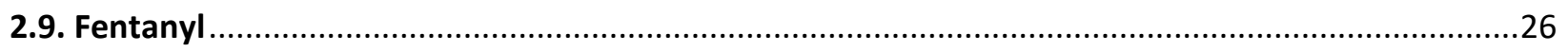

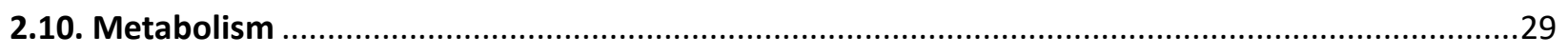

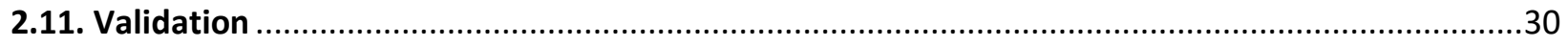

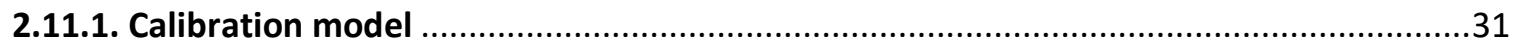

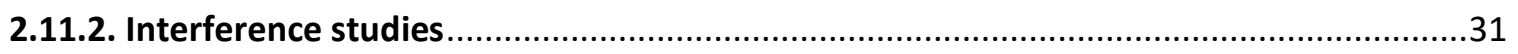

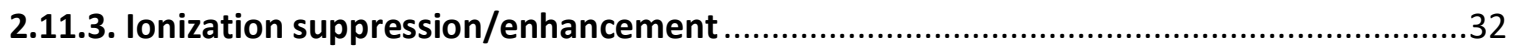

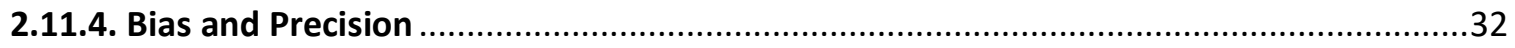

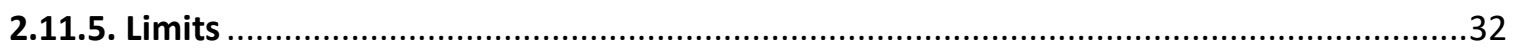

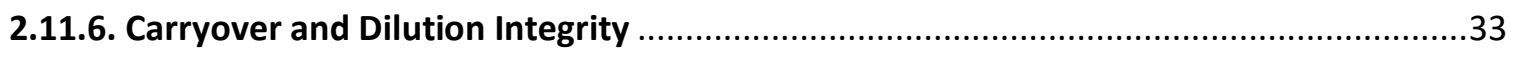

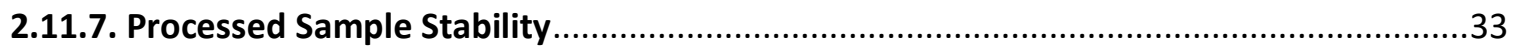

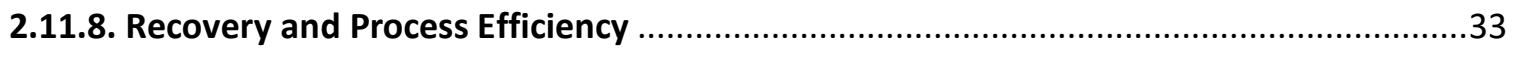

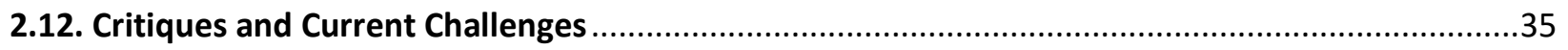


Chapter 3: Development and Application of QuEChERS Extraction Method for the Quantitation of Fentanyl and Metabolites from Liver Tissue.............................................39

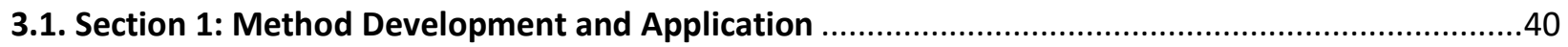

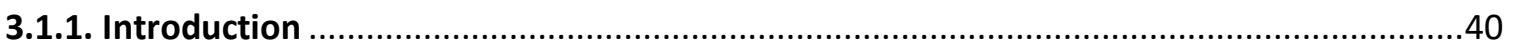

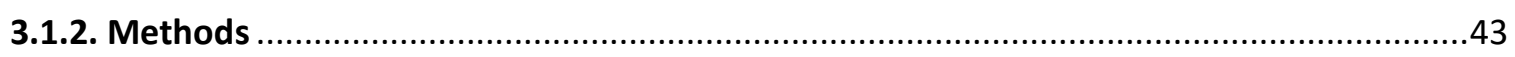

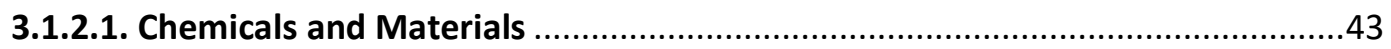

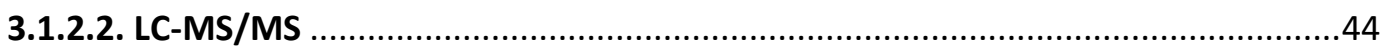

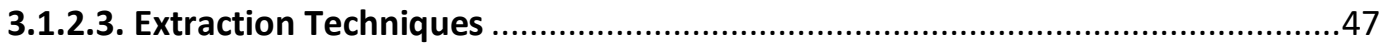

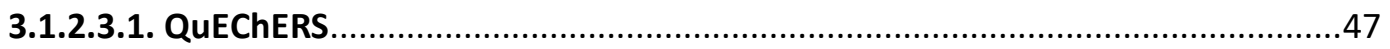

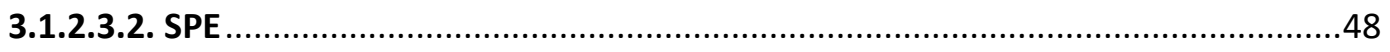

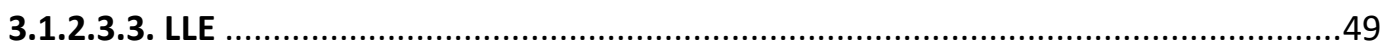

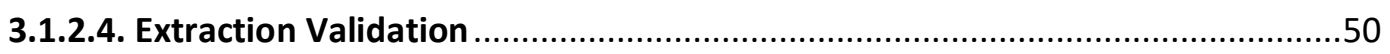

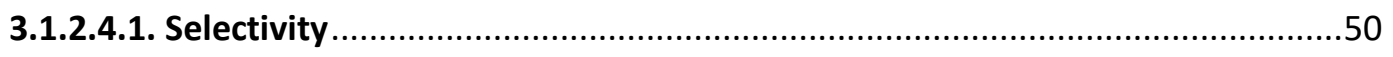

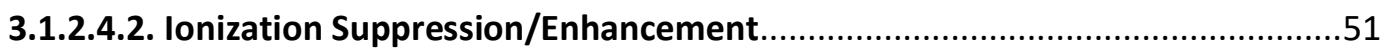

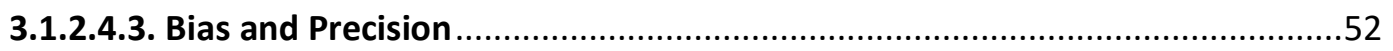

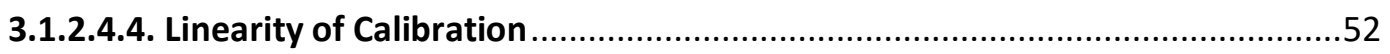

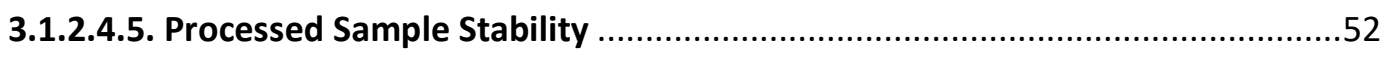

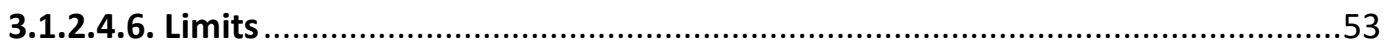

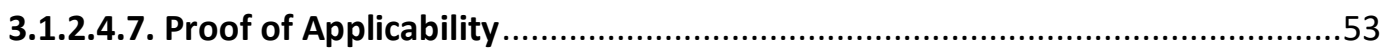

3.1.2.4.8. Comparison of Extraction Techniques (Recovery, Matrix Effects, and Process

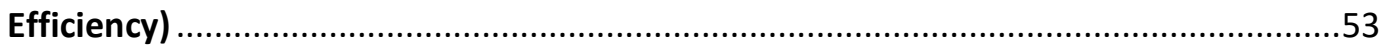

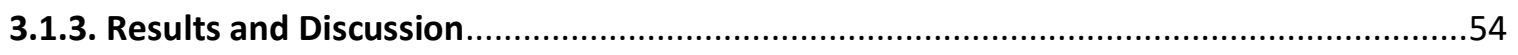

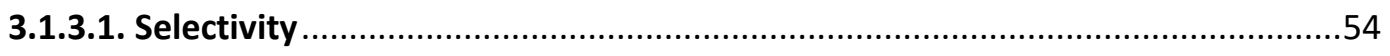

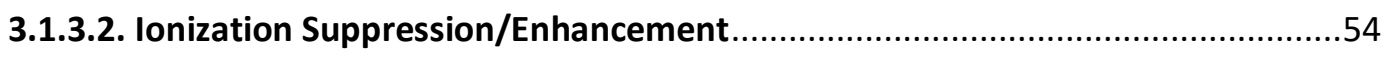

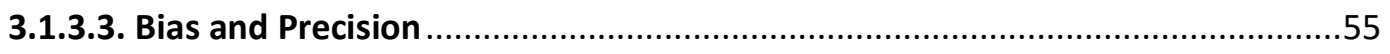

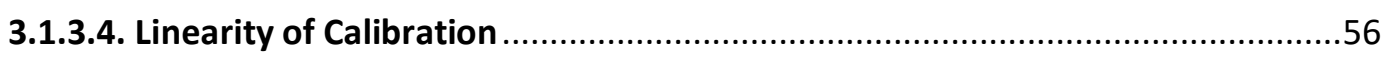

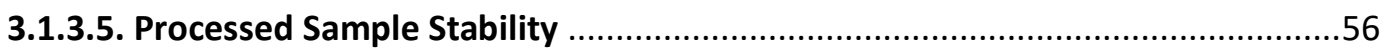

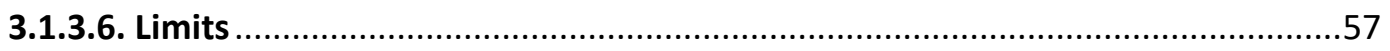

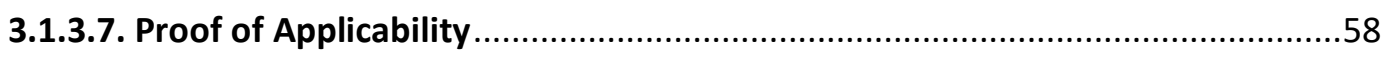

3.1.3.8. Comparison of Extraction Techniques .....................................................61

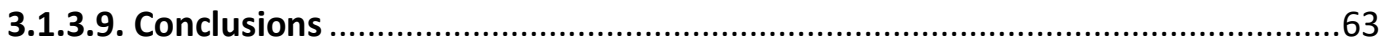

3.2. Section 2: Expansion of Method to 34 Fentanyl Analogs ......................................................64

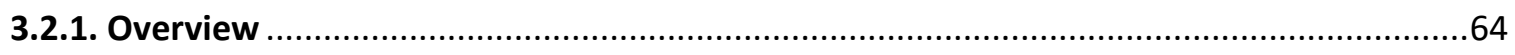

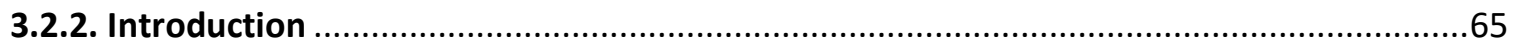

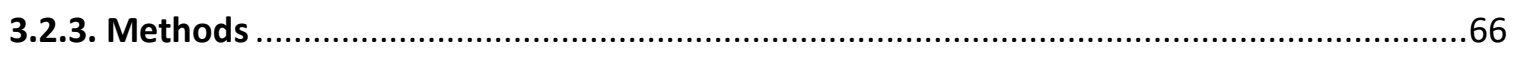

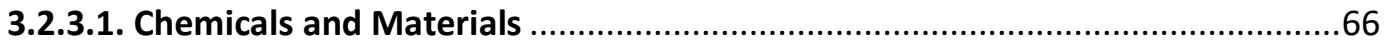

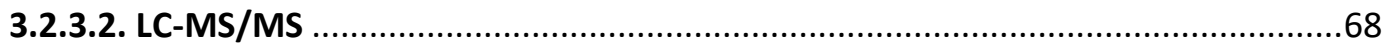

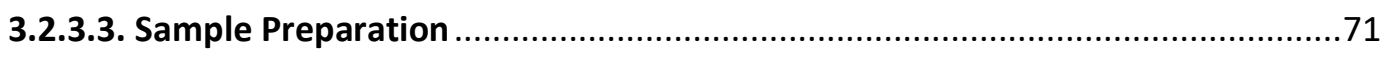

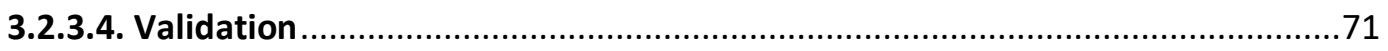

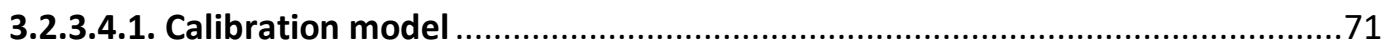

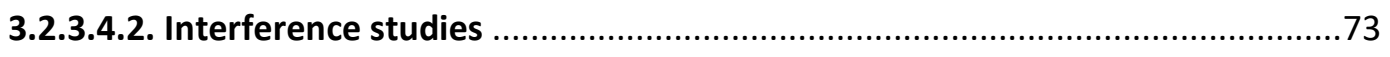

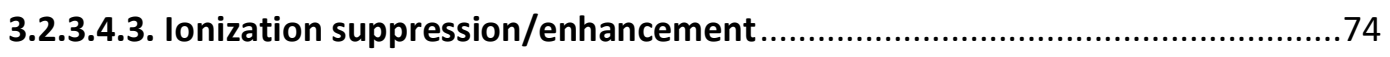

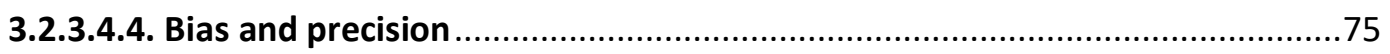




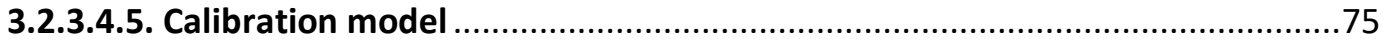

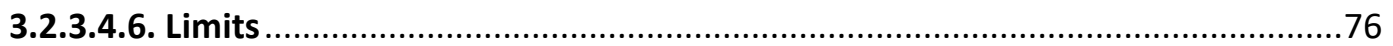

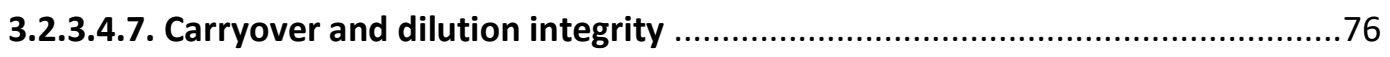

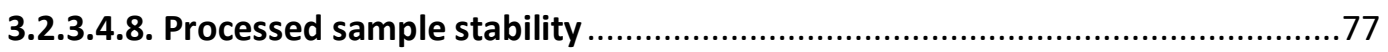

3.2.3.4.9. Recovery, matrix effects and process efficiency .......................................77

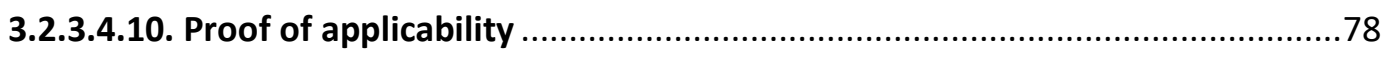

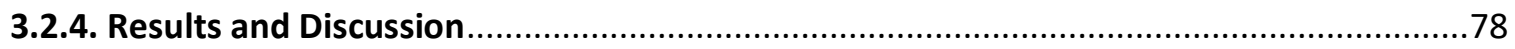

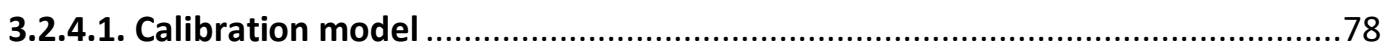

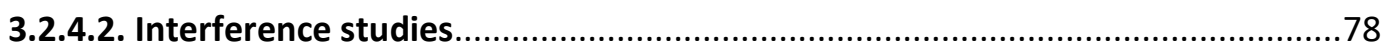

3.2.4.3. Ionization suppression/enhancement ..................................................... 79

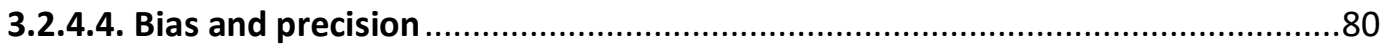

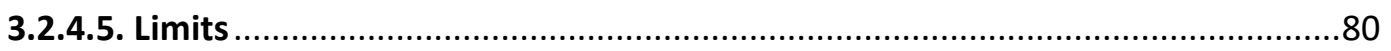

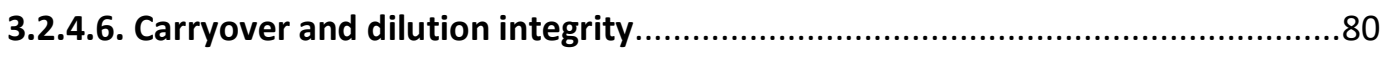

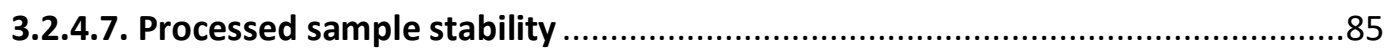

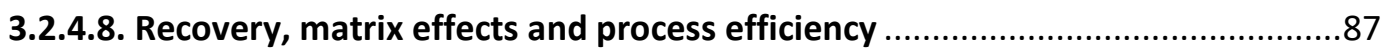

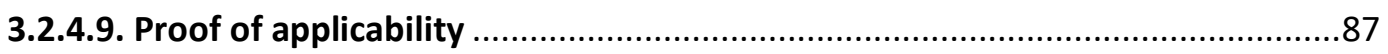

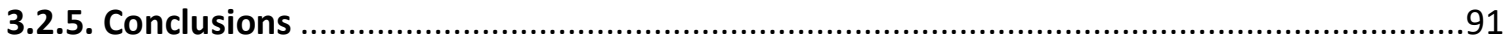

Chapter 4: Evaluation of the Effects of Fentanyl on Blowfly Larva and Application of QuEChERS Extraction Method for the Quantitation of Fentanyl and Metabolites from Larva and Pupa (Lucilia sericata) ..............................................................................93

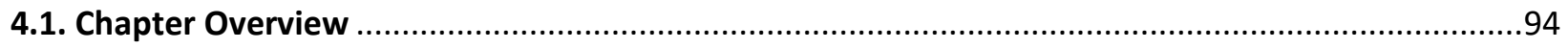

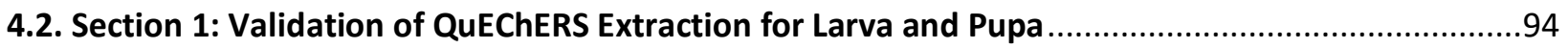

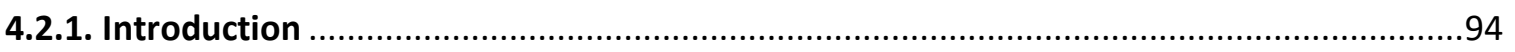

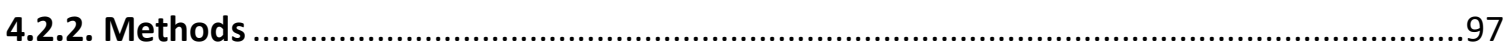

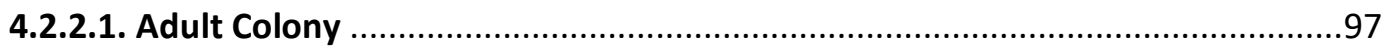

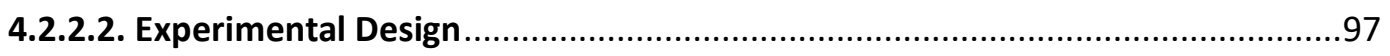

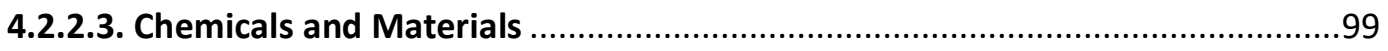

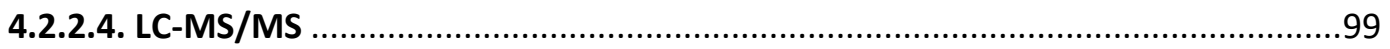

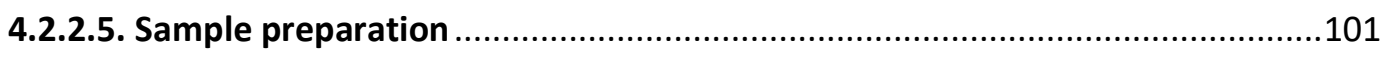

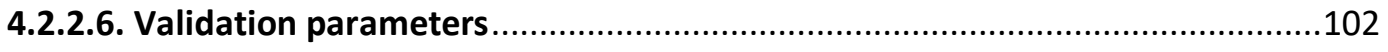

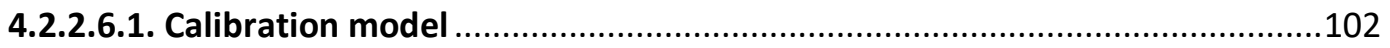

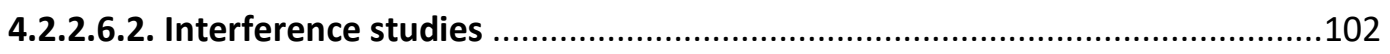

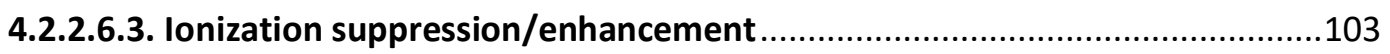

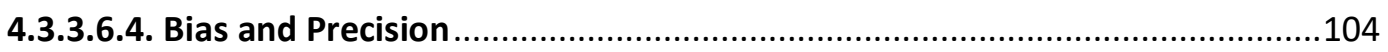

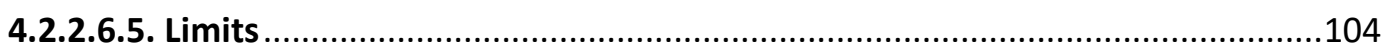

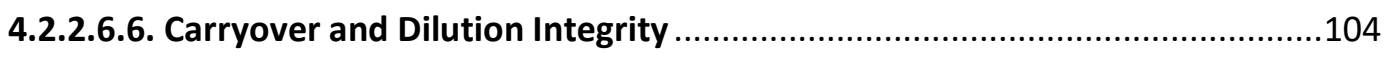

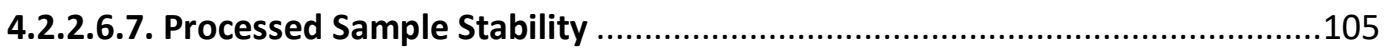

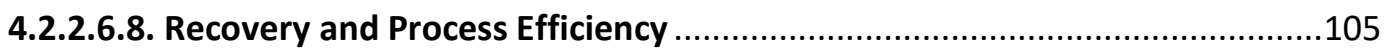

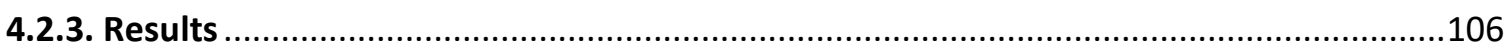

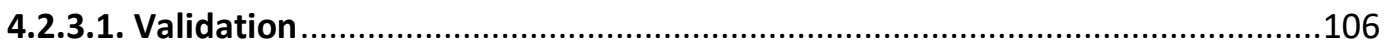

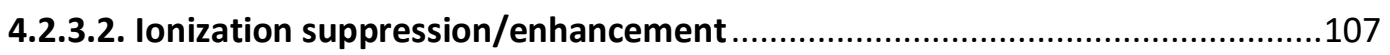

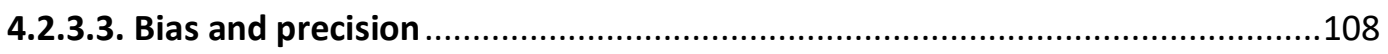

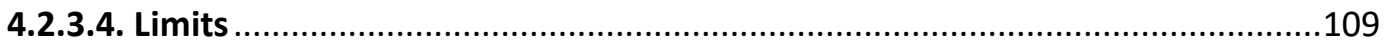

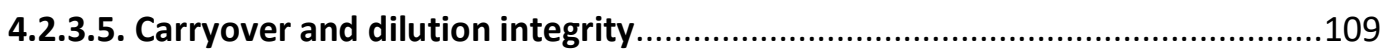




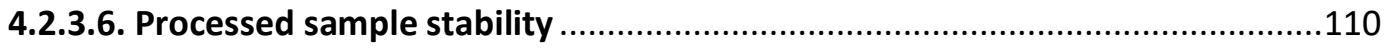

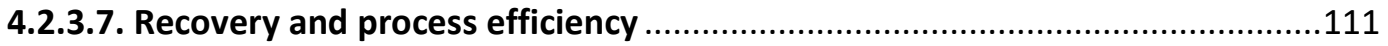

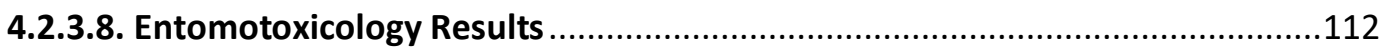

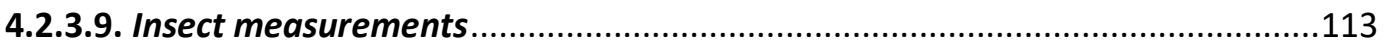

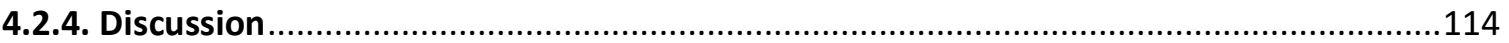

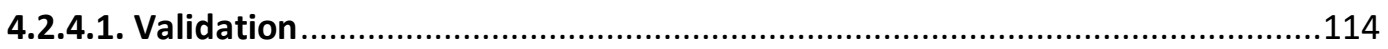

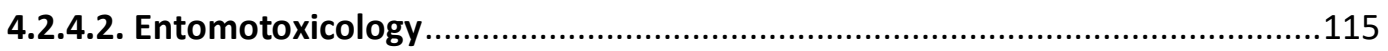

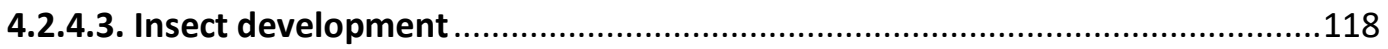

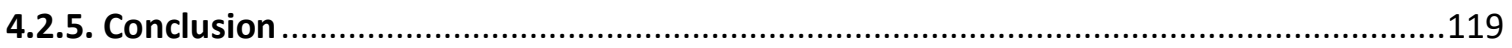

4.3. Section 2: Physical Effects of Fentanyl on Larva and Pupa (Lucilia sericata)................................120

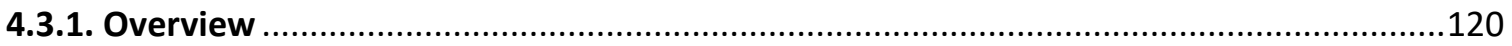

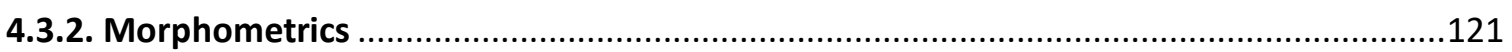

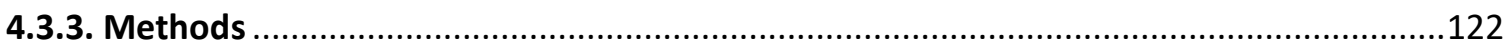

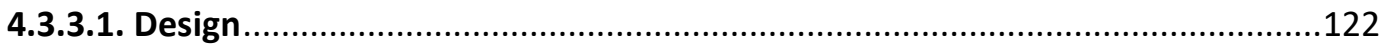

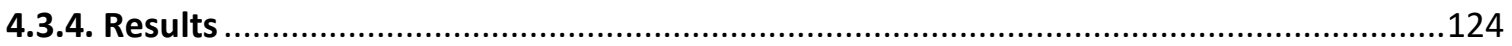

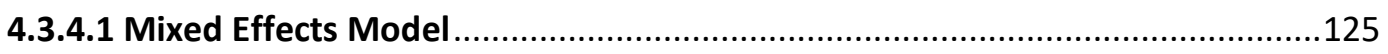

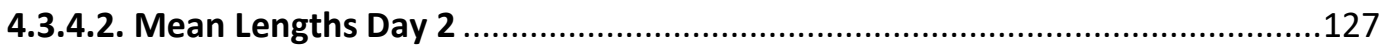

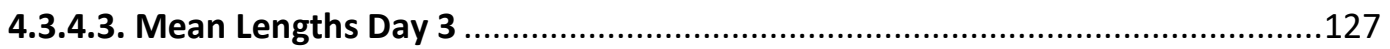

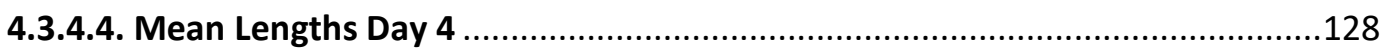

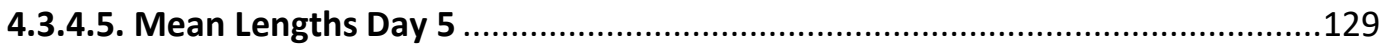

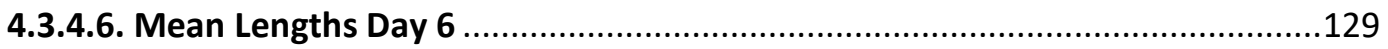

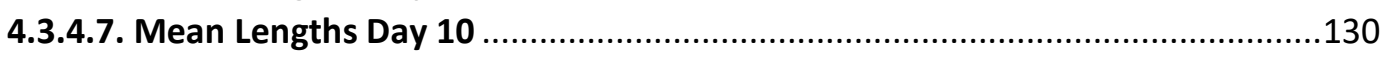

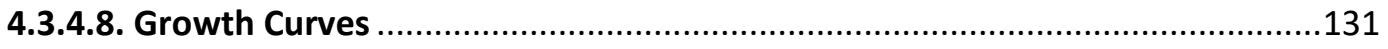

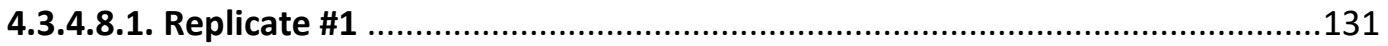

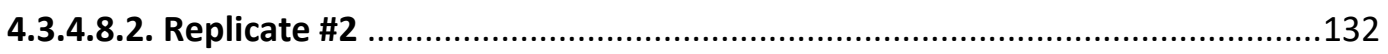

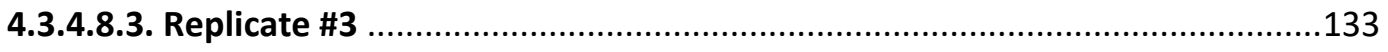

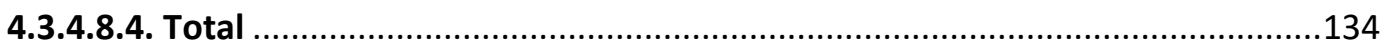

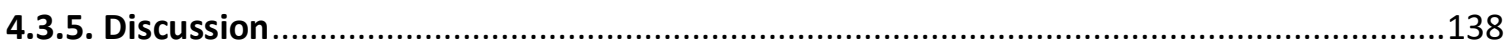

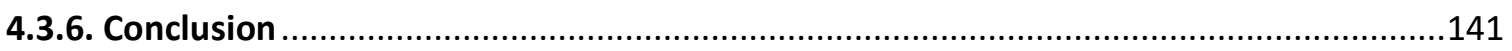

4.4. Section 3: Quantitation of Fentanyl and Metabolites from Larva and Pupa (Lucilia sericata).......142

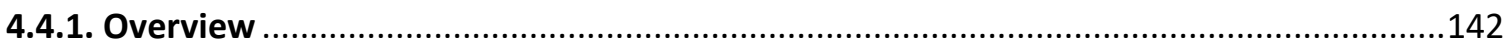

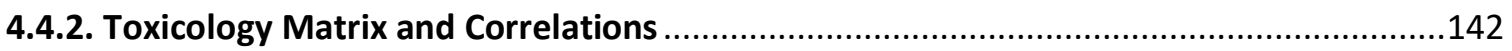

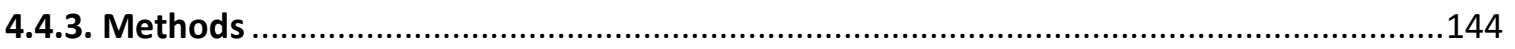

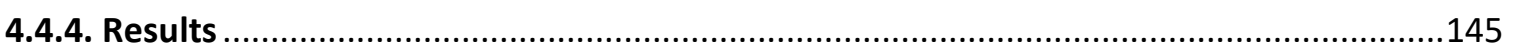

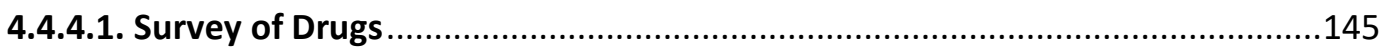

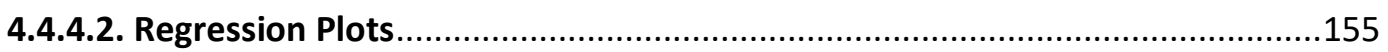

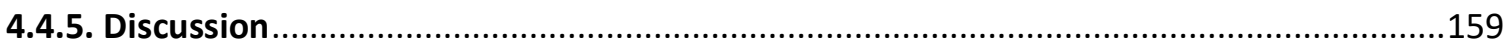

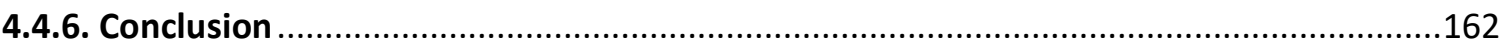

Chapter 5: Evaluation of the Effects of Fentanyl on Blowfly Adults and Application of

QuEChERS Extraction Method for the Quantitation of Fentanyl and Metabolites from Empty

Puparium and Adult Flies (Lucilia sericata) .............................................................. 164

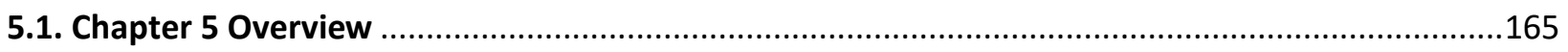

5.2. Section 1: Validation of QuEChERS Extraction for Empty Puparia and Adult Flies .......................165

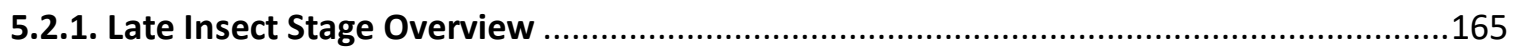




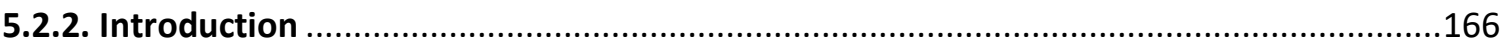

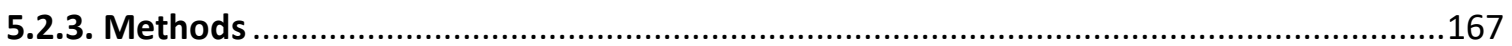

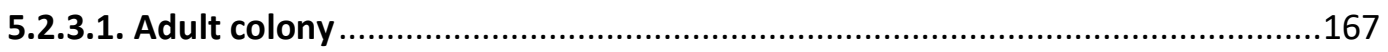

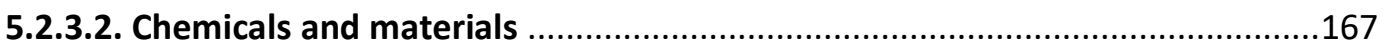

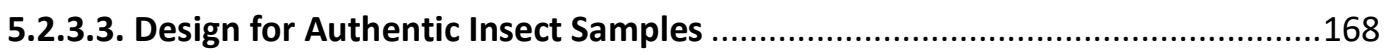

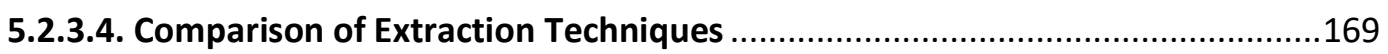

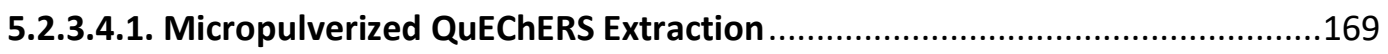

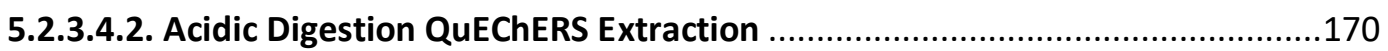

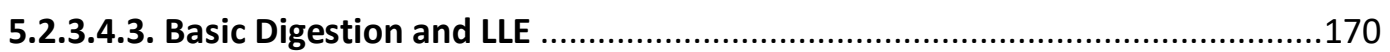

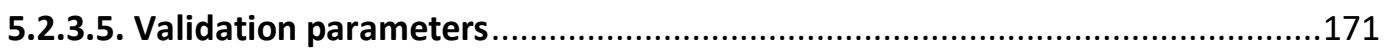

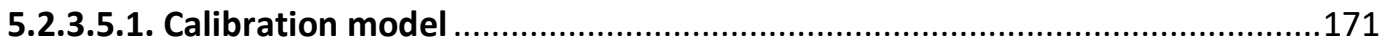

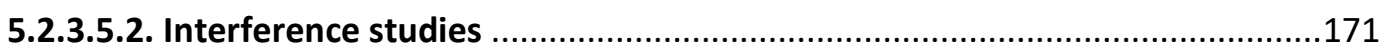

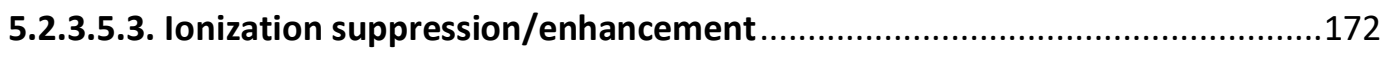

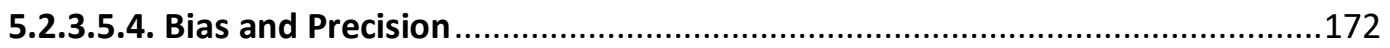

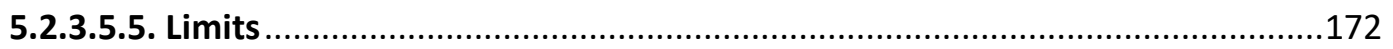

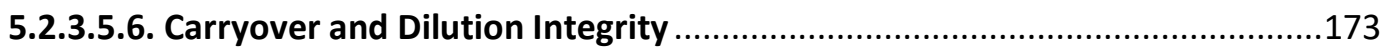

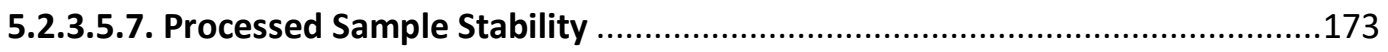

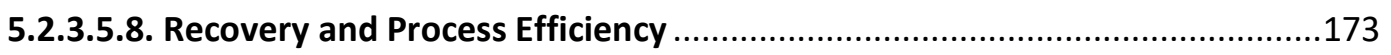

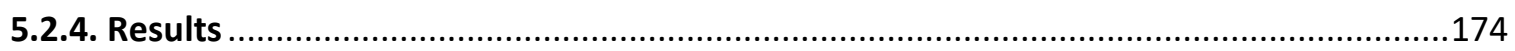

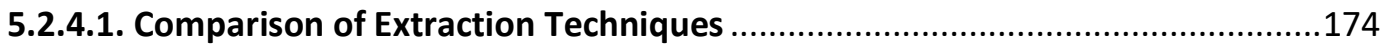

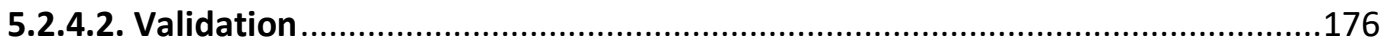

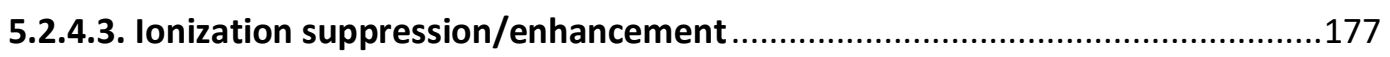

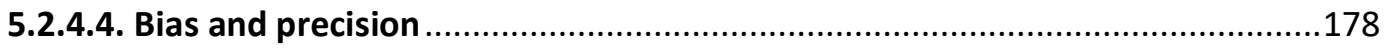

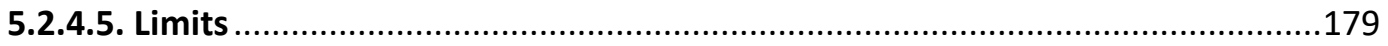

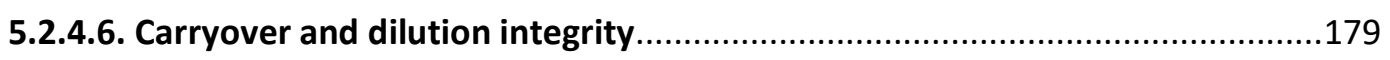

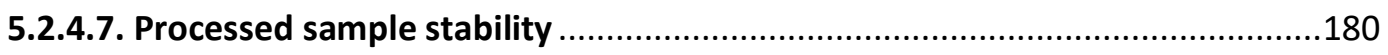

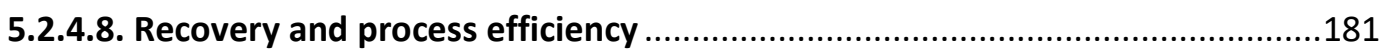

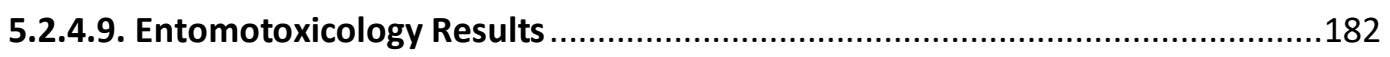

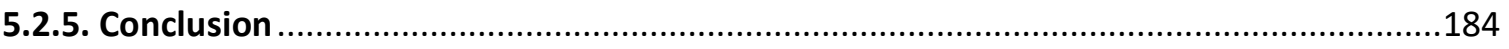

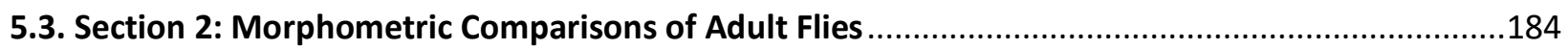

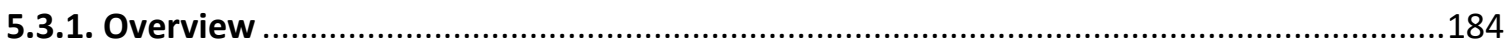

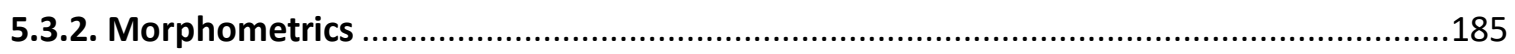

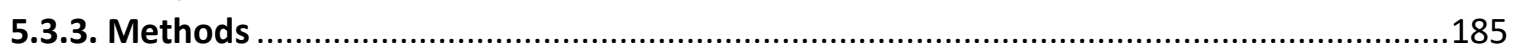

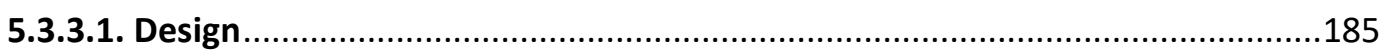

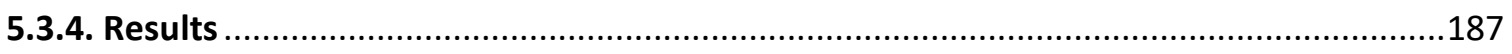

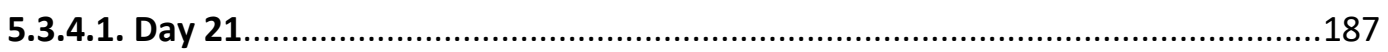

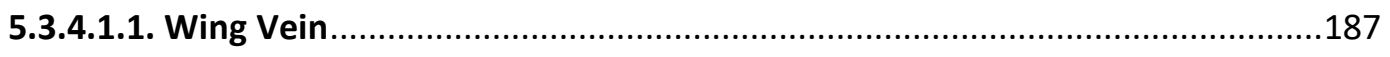

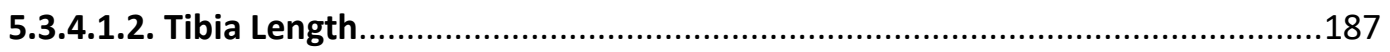

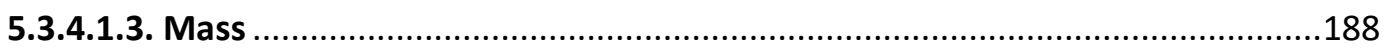

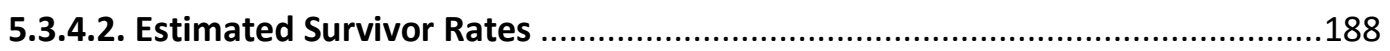

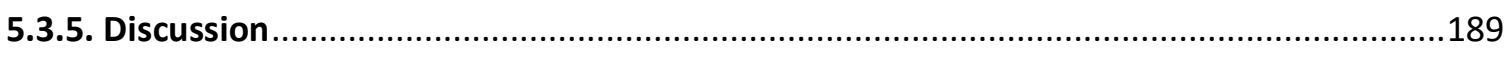

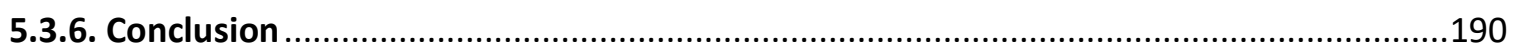

5.4. Section 3: Application of QuEChERS Extraction to Empty Puparia and Adult Flies .......................191

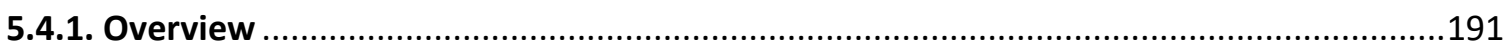


5.4.2. Toxicology Matrix and Correlations

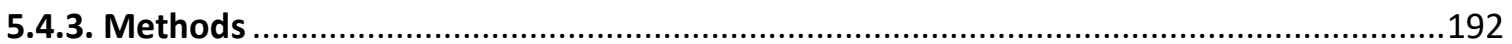

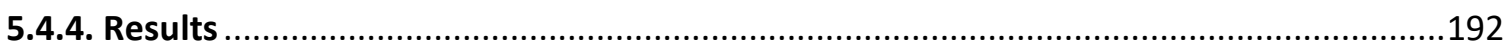

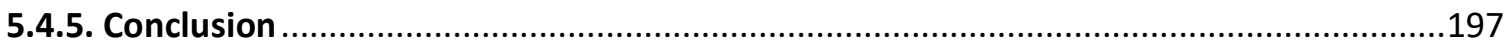

Chapter 6: General Conclusions............................................................................... 199

6.1. Chapter 3: Development and Application of QuEChERS Extraction Method for the Quantitation of Fentanyl and Metabolites from Liver Tissue. 200

6.2. Chapter 4: Evaluation of the Effects of Fentanyl on Blowfly Larva and Application of QuEChERS Extraction Method for the Quantitation of Fentanyl and Metabolites from Larva and Pupa (Lucilia sericata)

6.3. Chapter 5: Evaluation of the Effects of Fentanyl on Blowfly Adults and Application of QuEChERS Extraction Method for the Quantitation of Fentanyl and Metabolites from Empty Puparium and Adult

Flies (Lucilia sericata) .203

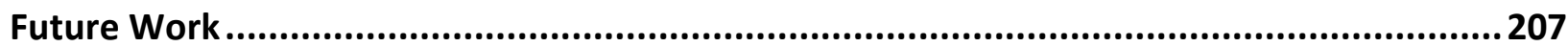

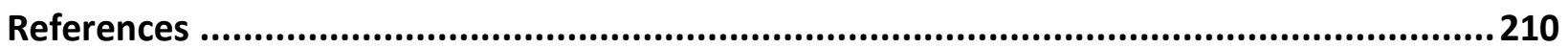

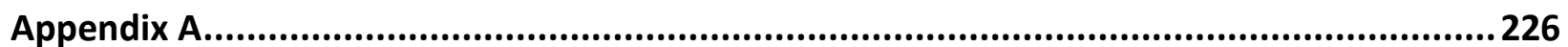




\section{List of Figures}

\section{Chapter 2}

Figure 2.1: Life cycle of the Green Bottle Fly Lucilia sericata...............................................................

Figure 2.2: Third instar larvae with posterior respiratory slits magnified. .............................................13

Figure 2.3: Image of a wandering third instar. Absence of feeding substrate in the crop is a clear

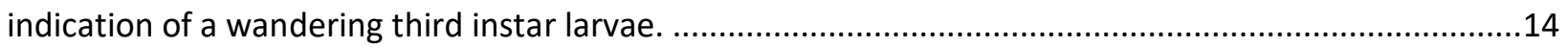

Figure 2.4: Diagram of the alimentary canal of a larval blow fly (25) ................................................15

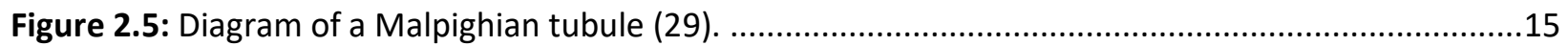

\section{Chapter 3}

Figure 3.1: Fragmentation predictions from ChemDraw for fentanyl quant transition (left) and the

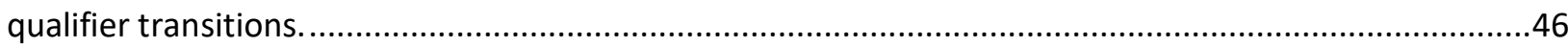

Figure 3.2: Images for the adapted homemade reaction chambers for the QuEChERS extraction. ..........48

Figure 3.3: Visual workflow for the QuEChERS extraction.................................................................48

Figure 3.4: Workflows for the LLE, SPE, and QuEChERS extraction protocols. ......................................50

Figure 3.5: LC-MS/MS chromatogram for the MRM transitions of the three target analytes. ..................54

Figure 3.6: Graphical representation of the stability of the high concentration for processed samples

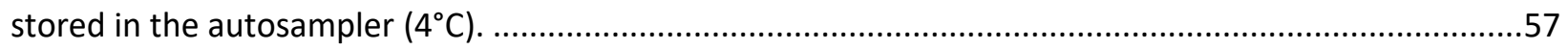

Figure 3.7: Box plot of quantitative results for the OCME case samples............................................60

Figure 3.8: Chromatogram of the 11 isobaric fentanyl analogs with norfentanyl and carfentanil for

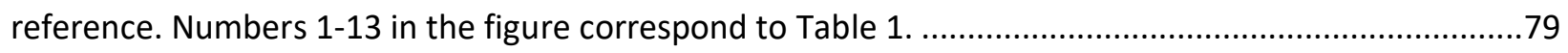

\section{Chapter 4}

Figure 4.1: Larvae (day 4) and pupae (day 12) measurements of length and width (mm).

Figure 4.2: Chromatographic separation of norfentanyl, $\beta$-hydroxy fentanyl, 4-ANPP, and fentanyl at the

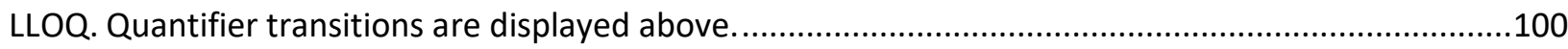

Figure 4.3: Fortified liver (25 g) aliquot (A) with 70-90 eggs placed (B)..........................................123

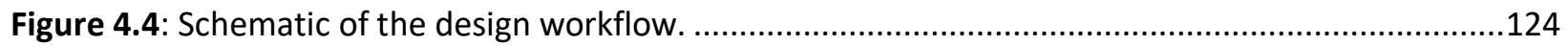

Figure 4.5: Plot of predicted means via the mixed effects model. ......................................................126

Figure 4.6: Comparison of mean lengths for insects collected on day 2 in replicates \#1-3...................127

Figure 4.7: Comparison of mean lengths for insects collected on day 3 for replicates \#1-3..................128

Figure 4.8: Comparison of mean lengths for insects collected on day 4 for replicates $\# 1-3 . \ldots \ldots \ldots \ldots \ldots \ldots . . . .128$

Figure 4.9: Comparison of mean lengths for insects collected on day 5 for replicates \#1-3...................129

Figure 4.10: Comparison of mean lengths for insects collected on day 6 for replicates \#1-3..................130

Figure 4.11: Comparison of mean lengths for insects collected on day 10 for replicates \#1-3................130

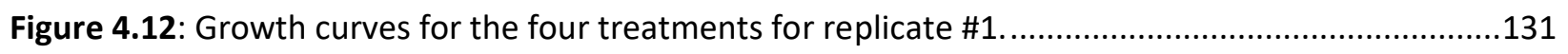

Figure 4.13: Growth curves for the four treatments for replicate \#2 ...............................................132

Figure 4.14: Growth curves for the five treatments for replicate \#3..................................................134

Figure 4.15: Growth curves for the five treatments for all three replicates combined...........................135

Figure 4.16: The maximum length measured from the larva collected on each day. ...........................136 
Figure 4.17: Box plot for the concentration of fentanyl quantitated for the single and pooled larva specimens collected from the high treatment liver on day 3 for all three replicates $(n=15)$.

Figure 4.18: Box plot for the concentration of fentanyl quantitated for the single and pooled larva specimens collected from the medium treatment liver on day 3 for all three replicates $(n=15)$.

Figure 4.19: Box plot for the concentration of fentanyl quantitated for the single and pooled larva specimens collected from the medium treatment liver on day 3 for all three replicates $(n=15)$.

Figure 4.20: Box plot for the concentration of fentanyl quantitated for the single and pooled larva specimens collected from the low treatment liver on day 3 for all three replicates $(n=15)$.

Figure 4.21: Box plot for the concentration of norfentanyl quantitated for the single and pooled larva specimens collected from the high treatment liver on day 3 for all three replicates $(n=15)$.

Figure 4.22: Box plot for the concentration of norfentanyl and 4-ANPP quantitated for the single and pooled larva specimens collected from the authentic treatment liver on day 3 for all three replicates $(n=5)$.

Figure 4.23: Regression plots for liver concentrations to larva concentrations for the single and pooled extractions collected on day 3 in replicate \#1.

Figure 4.24: Regression plots for liver concentrations to larva concentrations for the single and pooled extractions collected on day 3 in replicate \#2. 155

Figure 4.25: Regression plots for liver concentrations to larva concentrations for the single and pooled extractions collected on day 3 in replicate \#3.

Figure 4.26: Correlation plots for liver concentrations to larva concentrations for the single and pooled extractions collected on day 3 for all three replicates. 158

\section{Chapter 5}

Figure 5.1: Regression plot data for the liver to empty puparia fentanyl concentrations (left) and for liver to adult fentanyl concentrations (right). .183

Figure 5.2: Measurements for the costa vein in the fly wing and the tibia length .186

Figure 5.3: Comparison of mean wing vein length for insects collected on day 21 for replicates \#1-3...187 Figure 5.4: Comparison of mean tibia length $(\mathrm{mm})$ for insects collected on day 21 for replicates \#1-3. 188 Figure 5.5: Comparison of mean mass for insects collected on day 21 for replicates \#1-3. .188

Figure 5.6: Box plot for the concentration of fentanyl quantitated for the pooled empty puparia specimens collected from the authentic, medium, and high treatments on day 21 for all three replicates $(n=6)$

Figure 5.7: Box plot for the concentration of norfentanyl quantitated for the pooled empty puparia specimens collected from the authentic, medium, and high treatments on day 21 for all three replicates $(n=6)$

Figure 5.8: Box plot for the concentration of $\beta$-hydroxyfentanyl quantitated for the pooled empty puparia specimens collected from the low, medium, and high treatments on day 21 for all three replicates $(n=6)$.

Figure 5.9: Box plot for the concentration of fentanyl quantitated for the pooled adult fly specimens collected from the low, medium, and high treatments on day 21 for all three replicates $(n=6)$.

Figure 5.10: Box plot for the concentration of norfentanyl quantitated for the pooled adult fly specimens collected from the low, medium, and high treatments on day 21 for all three replicates $(n=6)$. 


\section{List of Tables}

\section{Chapter 2}

Table 2.1: Fentanyl and metabolites

\section{Chapter 3}

Table 3.1: MRM MS method parameters. .45

Table 3.2: List of 31 compounds evaluated for potential interferences.................................................51

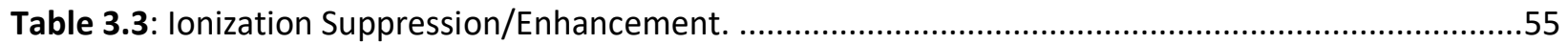

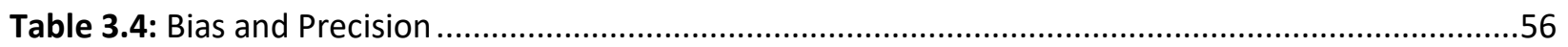

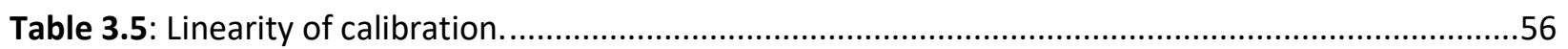

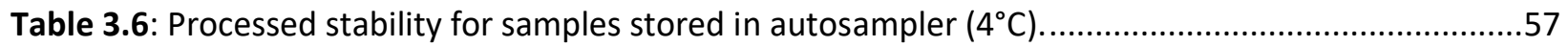

Table 3.7: Review of fentanyl liver concentrations $(\mu \mathrm{g} / \mathrm{kg})$. ............................................................59

Table 3.8: Comparison of beef and human liver extractions at low, med, and high concentrations $(n=5)$.

Table 3.9: Matrix effects, recovery, and process efficiency for the 3 extraction techniques $(n=5) \ldots \ldots \ldots . . .62$

Table 3.10: MS parameters for the 6470 Agilent system.........................................................................70

Table 3. 11: List of 31 compounds evaluated for potential interferences..............................................74

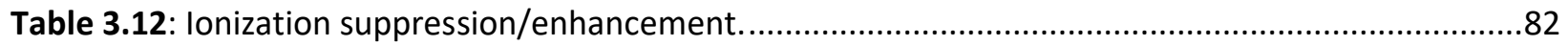

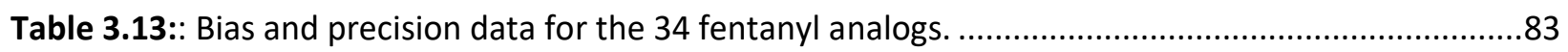

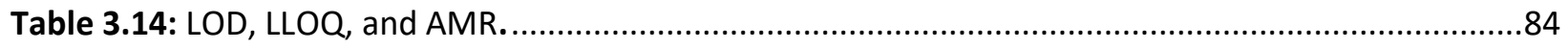

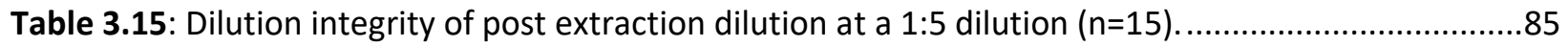

Table 3.16: Stability at a low and high concentration for the 34 analytes for processed samples stored in

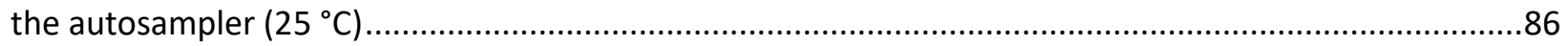

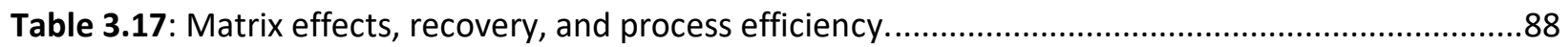

Table 3. 18: Authentic postmortem liver results. ..............................................................................90

\section{Chapter 4}

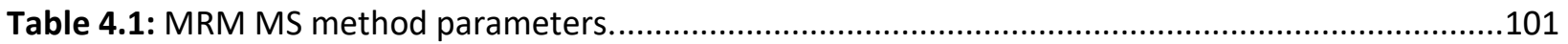

Table 4.2: List of 31 compounds evaluated for potential interferences................................................103

Table 4.3: LOD, LLOQ, and AMR for larvae and pupae extractions. ..........................................................106

Table 4.4: Ionization suppression/enhancement, recovery, and process efficiency for the larval and

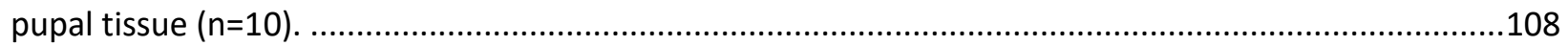

Table 4.5: The bias and precision validation data for larvae and pupae extractions $(n=15)$.................109

Table 4.6: Dilution integrity for the larvae and pupae extractions at a 1:5 dilution. .............................110

Table 4.7: Stability at a low and high concentration for the larvae and pupae extractions for processed

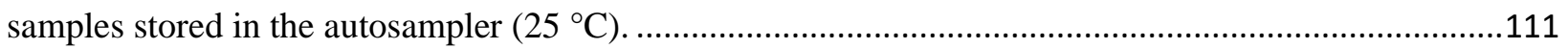

Table 4.8: Larvae and pupae fentanyl concentrations from day four and day twelve collections...........113

Table 4.9: Survivor rates for the day four and day twelve insect collections........................................114

Table 4.10: Percent stage over time for each life stage $\left(2^{\text {nd }}\right.$ instar larva, $3^{\text {rd }}$ instar larva feeding, $3^{\text {rd }}$ instar

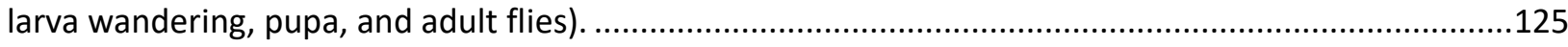

Table 4.11: Equations for the growth curve of the control group for each replicate and as a total. .......136 
Table 4. 12: Age estimations generated using the equations from Table 4.11. Estimated ages were compared to the actual day of collection to calculate a difference $(\Delta)$ age $(d)$

Table 4.13: Larval drug concentrations from day three collections for all three replicates....................147

Table 4.14: Larval drug concentrations from day six collections for all three replicates. ......................152

Table 4.15: Pupal drug concentrations from day 10 collections for all three replicates.........................154

Table 4.16: The linear regression equations for the regression plots with $R^{2}$ values...........................157

Table 4.17: Liver concentration estimation using larval concentration by linear regression...................158

\section{Chapter 5}

Table 5.1: Ionization suppression/enhancement, recovery, and process efficiency for the empty puparium tissue $(n=10)$

Table 5.2: Comparison for the three extraction techniques micropulverized QuEChERS extraction (MP), acidic digestion QuEChERS (acidic), and basic digestion LLE for the ability to extract fentanyl and metabolites from pooled $(n=16)$ authentic empty puparia specimens.

Table 5.3: LOD, LLOQ, and AMR for empty puparia and adult fly extractions.

Table 5. 4: Ionization suppression/enhancement, recovery, and process efficiency for the adults and empty puparium tissue $(n=10)$.

Table 5.5: The bias and precision validation data for adults and empty puparium extractions $(n=15)$...179

Table 5.6: Dilution integrity for the adult and empty puparium extractions at a 1:5 dilution.

Table 5. 7: Stability at a low and high concentration for the adult and empty puparium extractions for processed samples stored in the autosampler $\left(25^{\circ} \mathrm{C}\right)$....

Table 5.8: Concentrations of fentanyl and norfentanyl detected in the empty puparia and adult flies..183

Table 5.9: Percent estimated survivor rates for all the treatments and replicates combined. .189

Table 5.10: Gender distribution for the adult flies 189

Table 5.11: Empty puparia and adult drug concentrations from day 21 collections for all three replicates. 


\section{List of Abbreviations}

$\% \mathrm{CV}$ - Percent relative standard deviation

4-ANPP - Despropionyl fentanyl

AAFS - American Academy of Forensic Science

$\mathrm{ACN}$ - Acetonitrile

AMR - Analytical measurement range

ASB - Academy Standard Board

CNS - Central nervous system

CYP450 - Hepatic cytochrome P450

dMRM - Dynamic multiple reaction monitoring

d-SPE - Dispersive solid-phase extraction

EDDP - 2-Ethylidene-1, 5-Dimethyl-3, 3-Diphenylpyrrolidine

GC/MS - Gas chromatograph mass spectrometry

$\mathrm{HCl}-\mathrm{Hydrochloric} \mathrm{acid}$

HPLC - High performance mass spectrometry

IMF - Illicitly manufactured fentanyl

IRB - Institutional Review Board

IS - Internal standard

IWS - Intermediate working solution

LC-MS - Liquid chromatography mass spectroscopy

LC-MS/MS - Liquid chromatography tandem mass spectrometry

LLE - Liquid-liquid extraction

LLOQ - Lower limit of quantitation

LOD - Limit of detection

ME - Matrix effects

MgSO4 - Magnesium sulfate

MRM - Multiple reaction monitoring

MTBE - Methyl-tertbutyl ether 
$\mathrm{NaCl}$ - Sodium chloride

$\mathrm{NaOH}$ - Sodium chloride

NSO - Novel synthetic opioids

NSOs - Novel synthetic opioids

NVSS - National Vital Statistics System

OCME - Office of the Chief Medical Examiner

PE - Process efficiency

minPMI - Minimum PMI

PMR - Postmortem redistribution

PSA - Primary secondary amine

QuEChERS - Quick, Easy, Cheap, Effective, Rugged, and Safe

$\mathrm{RE}$ - Recovery of the extraction

REML - Restricted maximum likelihood

RIA - Radioimmunoassay

$\mathrm{s} / \mathrm{n}$ - Signal to noise

SPE - Solid phase extraction

SWGTOX - The Scientific Working Group for Forensic Toxicology

TLC - Thin layer chromatography

$V d-$ Volume of distribution

WVU - West Virginia University 
Chapter 1: Overview and Study Objectives 


\subsection{Project Overview}

In medico-legal death investigations, postmortem toxicology interpretations can be a crucial component in determining the cause of death. In a forensic postmortem toxicological workflow, typically various specimens are submitted for testing including: urine, blood, liver, kidney, and other complex biological tissues (1). Often, concentrations from one or more of the above specimen types are used to corroborate one another. Interpretation of these results generally depend on previously published work from the field. By investigating concentrations of drugs in various matrices in postmortem cases, the strength of toxicological interpretation increases especially involving cases with advanced decomposition. Insect tissue, usually larva or pupa, is widely accepted as a qualitative toxicology matrix with quantitative value being extremely limited. Insects, when there is open access to a cadaver, arrive shortly after death to colonize the body with eggs and persist through the entire decomposition process. This allows insect tissue to have a larger window of detection compared to most biological fluid or tissue samples. Another result of this natural association is that insects can be used for estimating the minimum postmortem interval $\left({ }_{\min } \mathrm{PMI}\right)$. Entomological estimation of ${ }_{\min } \mathrm{PMI}$ is rooted in the assumption that insects arrive shortly after death and that their development is primarily governed by temperature (2). Based on the physical size or stage of the insect and the thermal history, the age of an insect can be calculated at which the insect was collected $(2,3)$. The ${ }_{\min } \mathrm{PMI}$ can be a vital tool in death investigations by determining a minimum time that a cadaver has been exposed to insect development.

Under this scope, this research is focused on evaluating the developmental effects of fentanyl on immature stages of the blow fly Lucilia sericata (Meigen)) and the prevalence of fentanyl and metabolites from larval and pupal tissue after feeding on liver tissue with the presence of fentanyl.

This dissertation is presented in five main chapters, an overall conclusion section, and future work. Chapter 1 is a general overview with project objectives. In chapter 2 the biological elements of Lucilia sericata and forensic significance are presented including life cycle, distribution, and fundamentally how forensic entomology calculates ${ }_{\min }$ PMI. Toxicology background and significance presented in this chapter 
include extraction types, drugs of abuse, metabolism, and applications to insect tissues. General topics are presented in this chapter and are expanded in subsequent chapters.

Chapter 3 describes the evaluation and selection of the extraction protocol chosen to be utilized for the analysis of fentanyl and metabolites from complex biological matrices. Fentanyl is a potent $\mu$ opioid agonist with a high potential for abuse, addiction, and overdose. The detection and quantitation of fentanyl and metabolites from traditional matrices of blood, urine, vitreous, and liver tissue can be of vital importance in postmortem toxicology investigations. Blood can be susceptible to postmortem redistribution and often liver tissue is used to corroborate the blood concentrations. When blood or other fluids are no longer available or suitable for analysis, liver tissue is often the tissue selected by toxicologists due to its function in metabolism as well as its relative resistance to postmortem redistribution when compared to blood. As the decomposition process progresses, the complexity of the matrix and the condition of the sample can present a challenge for a toxicologist. This chapter focuses of the complexity of the liver matrix then later expansion to larva, pupa, empty puparium, and adult flies presented in chapters 4 and 5. Liver was selected as the matrix for comparison testing of liquid-liquid extraction (LLE), solid phase extraction (SPE), and a quick, easy, cheap, effective, rugged, and safe (QuEChERS) extraction with analysis by LC-MS/MS. The QuEChERS extraction was chosen for validation by the American Academy of Forensic Science Standard Board (ASB) Standard 036. After validation, the extraction was applied to 12 livers from authentic fentanyl intoxication investigations. The first part of this chapter was published in the Journal of Analytical Toxicology (4). The analysis of liver tissue was expanded to include a total of 34 fentanyl analogs and metabolites. This expansion was to help the field by providing an extraction technique coupled with LC-MS/MS analysis for the emerging synthetic opioids. A survey of the target analytes in 22 authentic samples was also completed. This chapter was published in the Journal of Analytical Toxicology (5). 
Chapter 4 describes the validation of a QuEChERS extraction of fentanyl and metabolites from larval and pupal tissue via LC-MS/MS. The analysis was validated under the ASB Standard 036. The overall design of the study is explained in this chapter including variable such as fortifying the liver tissue, concentrations, egg placement, collection times, physical characteristic observations, and toxicology analysis. The experimental design consisted of four treatment groups $(0,10,100$, and $350 \mu \mathrm{g} / \mathrm{kg})$ of fortified liver samples as the insect feeding media and insects were collected over seven time periods (2, $3,4,5,6,10$, and 21 days) for three replicates. The statistical analysis of the physical characters of length, width, and mass are presented in this chapter as well as the correlation plots for the concentration of the liver tissue to the concentration of the insect tissue for the early stages of larvae and pupae. The later insect stages of empty puparia and adult flies are presented in chapter 5 . Fentanyl and metabolites were detected in insects collected from the treatment groups and correlations for the insects collected on day 3 to liver concentrations were generated. Metabolism of fentanyl by the insect were observed with norfentanyl observed in the early feeding larva and $\beta$-hydroxyfentanyl observed in the later stages. Validation data from this chapter has been submitted to the Journal of Forensic Sciences.

Chapter 5 describes the ASB Standard 036 validation of a QuEChERS extraction of fentanyl and metabolites from empty puparium and adult tissue via LC-MS/MS. This chapter explores the later insect stages of the design presented in chapter 4 . With the matrix of empty puparium being highly keratinized, multiple extraction techniques were evaluated to establish the best extraction for the analysis of the empty puparium. The statistical analysis of the physical characters of wing vein length, tibia length, and mass are presented in this chapter as well as the correlation plots for the concentration of the liver tissue to the concentration of the insect tissue for the later stages of empty puparium and adult flies. Metabolism of fentanyl by the insect was observed in the later stages with $\beta$-hydroxyfentanyl observed in the empty puparia but not observed in the adult flies. Norfentanyl was detected in both the empty puparia and the adult flies. 
Overall, this study provided a methodology for detecting fentanyl and metabolites from all stage of the life cycle of $L$. sericata. In the feeding larva, a high correlation of insect fentanyl concentrations to liver concentrations was observed. Evidence of metabolism of fentanyl to $\beta$-hydroxyfentanyl and norfentanyl was observed as well as a trend of $\beta$-hydroxyfentanyl being observed in the later stages of the insect and norfentanyl observed throughout the insect's life cycle. An overall trend was not observed but the treatment of fentanyl in the liver had an impact on the growth of the insects that needs to be considered by entomologists when estimating PMI on individuals with fentanyl present. Major conclusions include the ability to extract and quantitate fentanyl and metabolites from all the insect life stages (larva, pupa, empty puparia, and adult flies), an observable correlation in the feeding larva fentanyl concentrations to liver concentrations, evidence of metabolism of fentanyl by L. sericata with persistence in all the insect life stages, and an impact of the presence of fentanyl in the feeding media even without a discernable trend.

\subsection{Project Design and Objectives}

\subsubsection{Overall Project Goals}

The overall aim of this study was to develop a comprehensive strategy to facilitate the extraction of novel synthetic opioids (NSO) including fentanyl and its major metabolites from highly complex biological specimens and to evaluate the effects fentanyl has on the growth and development of $L$. sericata. The isolation of fentanyl, metabolites, and analogs from liver tissue using modified QuEChERS is presented as a cheaper, cleaner, more efficient, and faster way to allow their detection via liquid chromatography tandem mass spectrometry (LC-MS-MS). Additionally, this novel procedure demonstrated supplementary capabilities to evaluate the effects of fentanyl on insect stages and provide assistance to interpretation on PMI when drugs are present in a cadaver. The informative power obtained throughout this research will have an impact in law enforcement investigations specially in those cases involving fentanyl where highly decomposed human remains are present by providing a technique to use insects as a toxicology 
matrix, a potential for a correlation of the quantitative concentration in the insects, and a word of caution for estimation of the postmortem interval when fentanyl is present in the corpse.

The specific aims of this project were to: 1) extract and quantify fentanyl and metabolites from complex matrices using QuEChERS to aid in forensic toxicology and medicolegal death investigations, 2) evaluate any growth effects fentanyl has on the development of the insects and the potential implications on the impact of PMI estimations, 3) draw conclusions on the ability to correlate the concentration of fentanyl and metabolites from the insect tissue to the initial concentration of the feeding media.

\subsubsection{Objectives and Tasks}

Objective 1: Development and validation of a QuEChERS extraction and LC-MS/MS quantitation method for fentanyl and metabolites from human liver tissue, larvae, pupae, empty puparium, and adult insects.

Task 1.a: Optimization of LC-MS/MS parameters for selected analytes (fentanyl, norfentanyl, 4-ANPP, and $\beta$-hydroxyfentanyl) including optimization of precursor ions, product ions, fragmentor power and collision energy. An Agilent 1290 Infinity II LC system coupled with an Agilent 6470 tandem mass spectrometer was used in this study.

Task 1.b: QuEChERS extraction and LC-MS/MS analysis method validation following ASB standard guidelines for accuracy, precision, and reproducibility. Recoveries and matrix effects for each media were evaluated to ensure the extraction of the target compounds is effective.

Task 1.c: Comparison of extraction efficiencies for tissue analysis between liquid-liquid extraction (LLE), solid phase extraction (SPE), and QuEChERS. The extraction methods were evaluated at two concentrations (high and low) with a minimum of 5 replicates at each concentration for each method (30 spiked-liver samples and 15 control liver samples).

Task 1.d: Fortified tissue treatment regime. For the fortified tissue, a control (no drugs added) and three treatments were chosen to be performed in triplicate. The treatments consisted of a low concentration $(10 \mu \mathrm{g} / \mathrm{kg})$, a medium $(100 \mu \mathrm{g} / \mathrm{kg})$ and a high $(350 \mu \mathrm{g} / \mathrm{kg})$ based on casework review. Treatments were chosen to simulate the various types of potential death and resulting decomposition of 
a person from non-fentanyl related death, mixed drug fentanyl related death and fentanyl related death. A mass of 70-90 eggs were added to each treatment. On the day that eggs are added to the treatment, approximately $2 \mathrm{~g}$ of tissue was taken for LC-MS/MS analysis and recorded as Day 0.

Task 1.e: Sampling insect tissue for the quantitation of fentanyl and metabolites. Sampling was performed at four times: at 96 hours (feeding larvae), 144 hours (wandering larvae), 192 hours (pupae), and emergence into adult for four treatments (each at $0,10,100,350 \mu \mathrm{g} / \mathrm{kg}$ ). Figure 2.9 displays the design flow. In each treatment 70-90 eggs of Lucilia sericata were placed and the treatments were replicated in triplicate. For each day that insects were sampled, $2 \mathrm{~g}$ of tissue from the feeding media was taken for analysis. A modified QuEChERS extraction was used for feeding liver and insect tissue. Insect tissues were extracted as single samples ( 5 individual insects) and as pooled specimens ( 5 sets, each of 4 pooled insects), each in triplicate. The same samples were used for tasks $2 a, 2 b$ and $3 a$.

Task 1.f: Extraction of fentanyl and metabolites from casework liver tissue from authentic fentanyl related overdose victims. Samples were provided as part of our collaboration with the Office of the Chief Medical Examiner (OCME), to serve as our validation set.

Objective 2: Determination of any significant effects of fentanyl on athe growth and development of the insects.

Task 2.a: Evaluation of the effects of rearing the insects on fentanyl infused liver tissue.

For the development effects, each of seven factors had a treatment of fentanyl concentration in the liver tissue the insects were reared on consisting of one of the four levels: $0,10,100,350 \mu \mathrm{g} / \mathrm{kg}$ with 0 $\mu \mathrm{g} / \mathrm{kg}$ as the control group. Seven sampling times (factors) at 48 hours, 72 hours, 96 hours, 120 hours, 144 hours, pupa (192 hours), and emergence were evaluated (figure 2.9). In each treatment 70-90 eggs of Lucilia sericata were placed and the treatments were replicated in triplicate. 

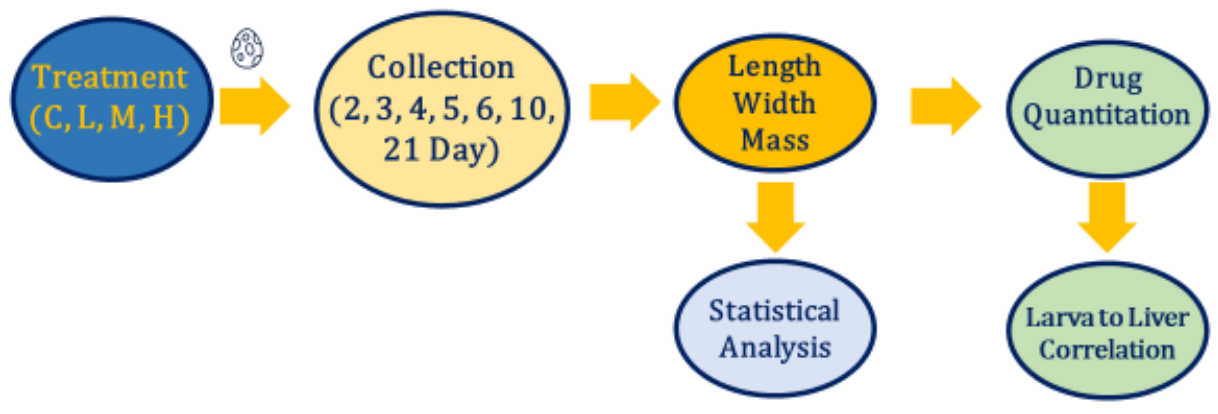

Figure 2.1: Design workflow.

Task 2.b: Recording of physical characteristics of insects: For all the insect stages, physical characteristics were recorded to evaluate the effects each treatment had on growth. To determine the effects of fentanyl on the growth and development of the blow flies, the mass, and percent stage (first instar, second instar, third instar, pupa, or adult) of the insect were collected including: the feeding instar (48 hours, 72 hours, and 96 hours), wandering third instar (120 hours and 144 hours), and pupae (240 hours). The percent stage of the insects was monitored at seven timings to evaluate the effects of fentanyl of the growth rate of the insects. Measurements for mass were recorded for the larval and pupa, empty puparium, and adult fly specimens. For each treatment and time sampling, the entire group of insects were culled and collected.

Task 2.c: Statistical analysis for evaluation of growth and development effects of fentanyl on the insects. A nested ANOVA test was utilized to determine if the control and treatment groups differ. Further Tukey-HSD tests determined which treatments were significantly different and due to sample size differences, restricted maximum likelihood (REML) was applied. Also, a mixed effect model to incorporate the length, width, and mass as a complete model was applied (equation below). These statistical tests determined if the drug treatments have any significant effects on the insect's development.

$$
\widetilde{\boldsymbol{Y}}=\widetilde{\boldsymbol{\mu}}+\delta_{j}+\tau_{i}+(\delta \tau)_{j: i}+\left(\begin{array}{ccc}
1 & 0 & 0 \\
0 & 1 & 0 \\
0 & 0 & 1
\end{array}\right)\left(\begin{array}{c}
\tau_{i: L} \\
\tau_{i: M} \\
\tau_{i: W}
\end{array}\right)+\left(\begin{array}{ccc}
1 & 0 & 0 \\
0 & 1 & 0 \\
0 & 0 & 1
\end{array}\right)\left(\begin{array}{c}
\delta_{j: L} \\
\delta_{j: M} \\
\delta_{j: W}
\end{array}\right)+\left(\begin{array}{ccc}
1 & 0 & 0 \\
0 & 1 & 0 \\
0 & 0 & 1
\end{array}\right)\left(\begin{array}{c}
(\delta \tau)_{j: i: L} \\
(\delta \tau)_{j: i: M} \\
(\delta \tau)_{j: i: W}
\end{array}\right)+\widetilde{\mathbf{E}_{\boldsymbol{\rho}}}+\widetilde{\mathbf{E}_{\boldsymbol{u}}}
$$




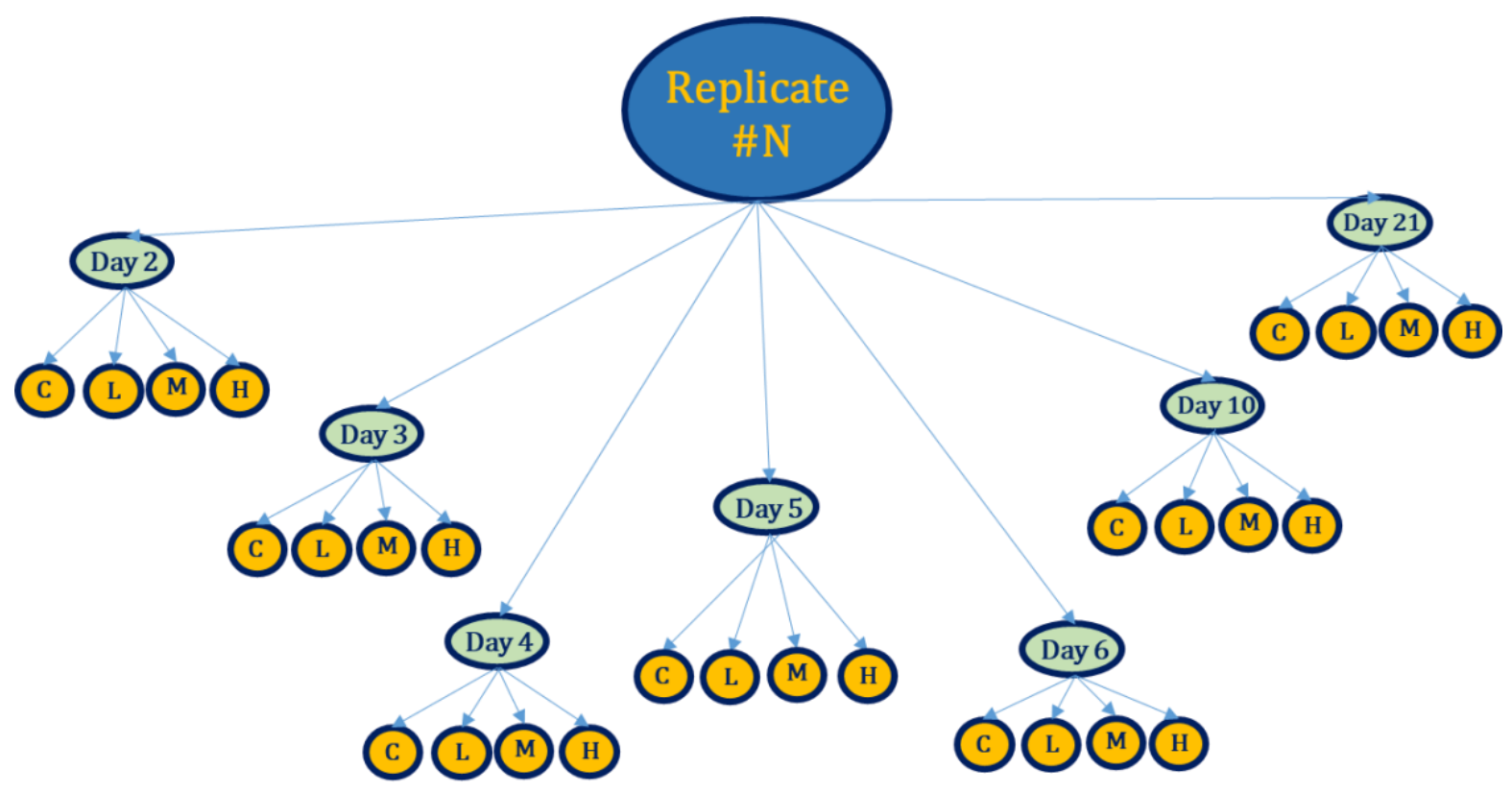

Figure 2.2: Nested ANOVA design for a single replicate $(n=3)$.

Task 2.d: Comparison of a subset of insects reared on authentic overdose liver specimens. For replicate \#3, a liver specimen with an authentic fentanyl concentration (non-fortified liver) was homogenized and added as fifth treatment group. The number of eggs, insects collected, morphometric observations, and toxicology extractions were the same as the other treatment groups. Concentrations of the authentic livers were determined before the eggs were placed.

Objective 3: Determination of fentanyl and metabolites concentration correlation found in the insect tissue to fentanyl concentrations in human tissue the insects reared on.

Task 3.a: Comparisons of concentrations extracted from the insect to the initial infused concentrations of the liver tissue. Using the concentrations extracted from each insect life stage of the various treatments (task 2a), a correlation analysis will be conducted to determine if there was consistent insect to human tissue ratios. 
Chapter 2: Introduction and Literature Review 


\subsection{Introduction}

This chapter provides a review of the current literature on forensic entomology and forensic entomotoxicology including the biological factors, life cycle of the investigated insect, estimation of minimum postmortem interval, insects as toxicology specimen, and the impact drugs can have on the growth of insects. Background information of the drugs, metabolism, and matrices of interest are presented here. The fundamentals of toxicology extraction and analysis methods are also presented in this chapter.

\subsection{Forensic Entomology}

The term forensic entomology describes the study of insects and other arthropods in the course of a criminal investigation $(6,7)$. Once algor mortis (the cooling of the body to ambient temperature after death) has reached completion, the estimation of the time since death or postmortem interval (PMI) is attributed to the field of forensic entomology $(7,8)$. Specifically, medicolegal entomology is the branch of forensic entomology that focuses on the estimation of the minimal postmortem interval $(\min P M I)$ for decomposing remains of humans or animals in which a criminal case is pending or if the death is under suspicious circumstances $(7,9)$. It should be noted that insect age estimation of ${ }_{\min } \mathrm{PMI}$ and PMI are not perfectly interchangeable, as PMI includes the potential for a lag time for insect colonization that $\min _{\mathrm{PMI}}$ using insect age cannot account for.

\subsubsection{Biological Factors}

Arthropods are the largest biological group and are found in environments all around the globe $(10,11)$. Arthropods are poikilothermic, meaning they do not regulate their body temperature and are regulated by the environmental temperature $(12,13)$. This reliance on the environment for thermal regulation creates a predictable growth pattern for arthropods $(12,14)$. The arthropod of most significance to forensic investigation are from the orders of Diptera and Coleoptera $(10,15)$. Necrophagous insects that are attracted to human decomposition are commonly the focus for entomological death investigations $(10,14,15)$. Necrophagous flies (Diptera) are common insects to first 
arrive and beetles (Coleoptera) often arrive later to feed on fly larva or in the later stages to feed on dried remains $(10,15)$.

Blow flies (Diptera: Calliphoridae) and Flesh flies (Diptera: Sarcophagidae) are typical first insect colonizers for decomposition with blow flies often the initial insects to arrive $(16,17)$. Initial colonization is a key component to calculating ${ }_{\min } \mathrm{PMI}$ and complete PMI. The sooner an insect arrives, the shorter the lag time between colonization and the time a person dies. Blow flies lay eggs in aggregation and the larva develop as an aggregation $(18,19)$. Feeding as an aggregation facilitates exodigestion, food intake, and assists with thermal regulation (18-20). The holometabolous life stages of blow flies begin with oviposition of an egg and progress to first instar, second instar, feeding third instar, wandering or migrating third instar, pupation, and finally emergence as adults (Figure 2.1$)(2,21,22)$. Through the blow fly's life cycle, behavioral and physical changes occur to the insect (23). The life of the blow fly begins with oviposition of the egg directly on or near the cadaver $(17,23,24)$. Once an egg hatches, the larva begins feeding and progresses through three stages of first, second, and third instar $(17,21)$. The progression through the larva instars is distinguished by molting events and an increase in posterior respiratory slits (Figure 2.2) (21, 23). Once a third instar larvae feeds enough, a behavioral change in the insect occurs as the larvae stops feeding and begins to leave the feeding substrate to begin pupation. This developmental stage is called a wandering or migrating third instar larvae $(21,23,24)$. Once the larvae stops feeding and begins to migrate, there is a decrease in length of the insect as it begins the process of pupation $(21,23)$. After pupation, the insect will emerge for the pupa casing as an adult fly while leaving behind an empty puparium. 


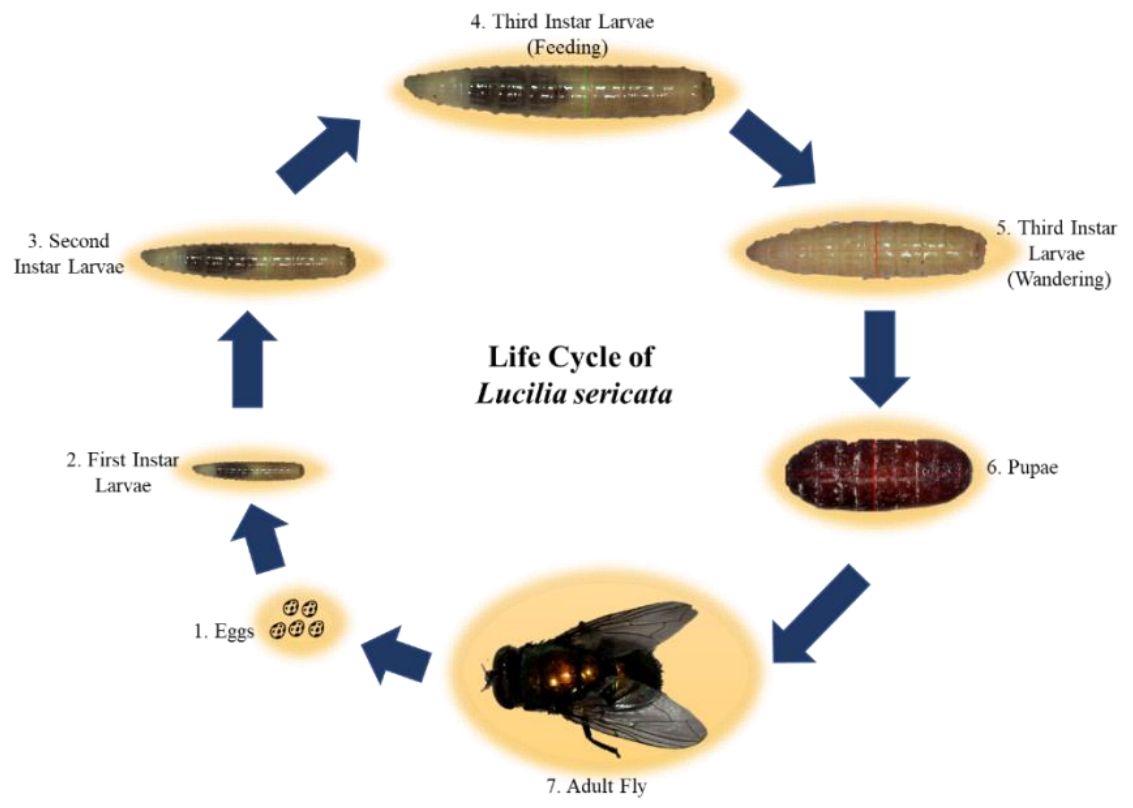

Figure 2.3: Life cycle of the Green Bottle Fly Lucilia sericata.

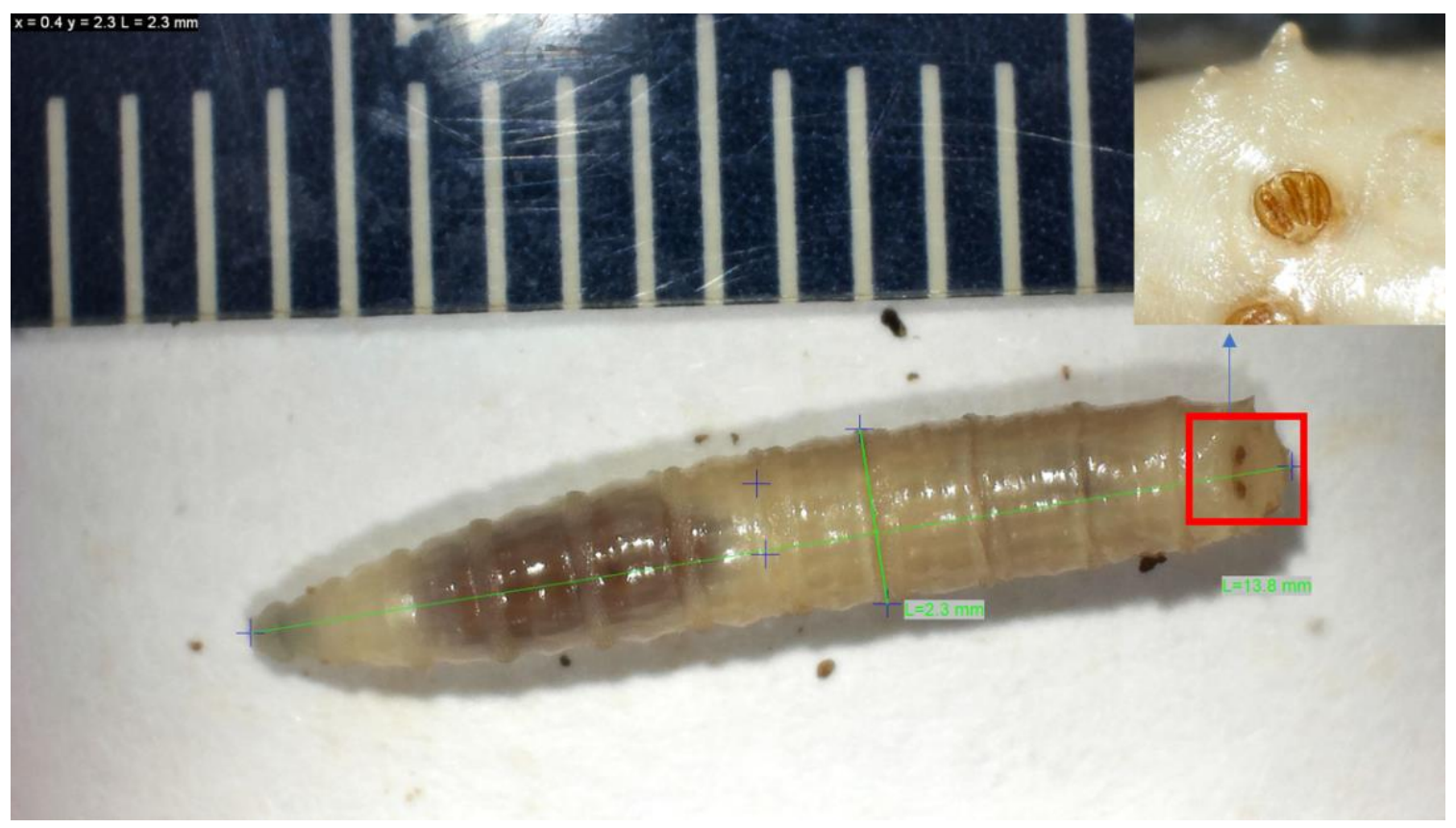

Figure 2.4: Third instar larvae with posterior respiratory slits magnified. 


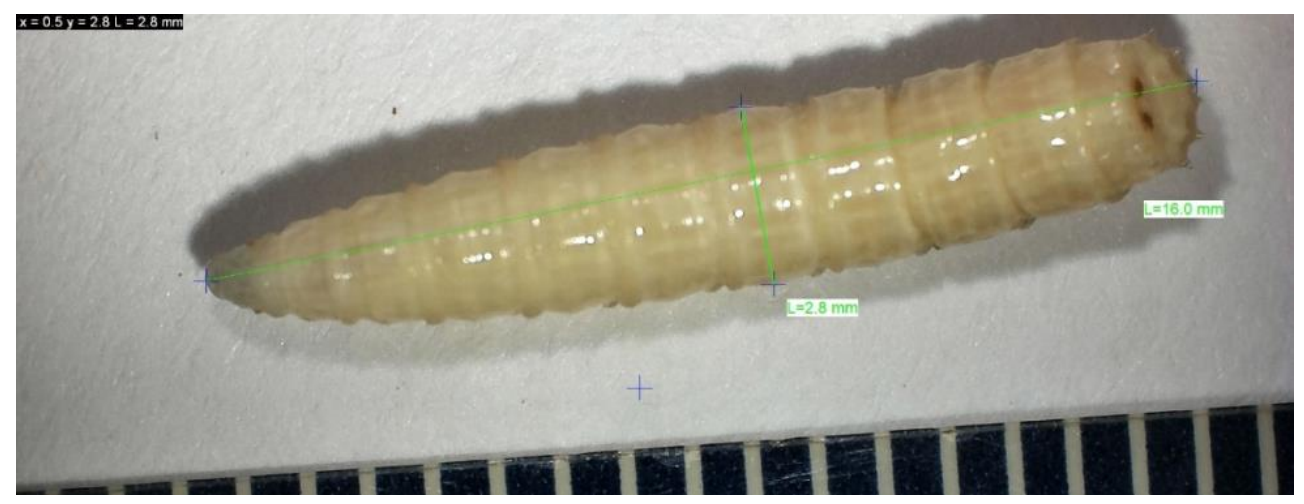

Figure 2.5: Image of a wandering third instar. Absence of feeding substrate in the crop is a clear indication of a wandering third instar larvae.

The larva of the blow fly will feed in an aggregation, which helps with digestion and thermal regulation. The anatomy of the blow fly larva contains a complete alimentary canal starting with a mouth and mouth hooks with completion to hindgut and anus (25). The mouth of the larvae has no teeth however it does contain mouth hooks $(19,20,26)$. These mouth hooks help the larvae with locomotion, to pierce soft tissue, and to help the insect burrow deeper into the feeding substrate (18-20). The larva lack the ability to truly masticate and therefore the need to ingest the feeding media as liquefied substrate or in semi solid form $(18,19)$. To aid in the liquification of the tissues, the larva secrete enzymes, typically proteases or carbohydrases to facilitate the breakdown of the tissues $(18,19,26,27)$. The aggregation of the larva increases the breakdown of the tissue as more larva secrete enzymes, the faster the tissue liquefies and the faster the larva can feed $(18,19)$. As the larvae feeds, the liquefied tissue is sucked in through the mouth, and passes through the esophagus to the crop (19). After passing the crop, the next segment is the foregut, midgut, hindgut, ileum, colon, rectum, and finally anus (Figure 2.4) (19). At the junction of the midgut and hindgut is where the Malpighian tubules diverge (19). Malpighian tubules 
function to remove waste, exogenous materials, and any other ions from the insect $(19,28)$.

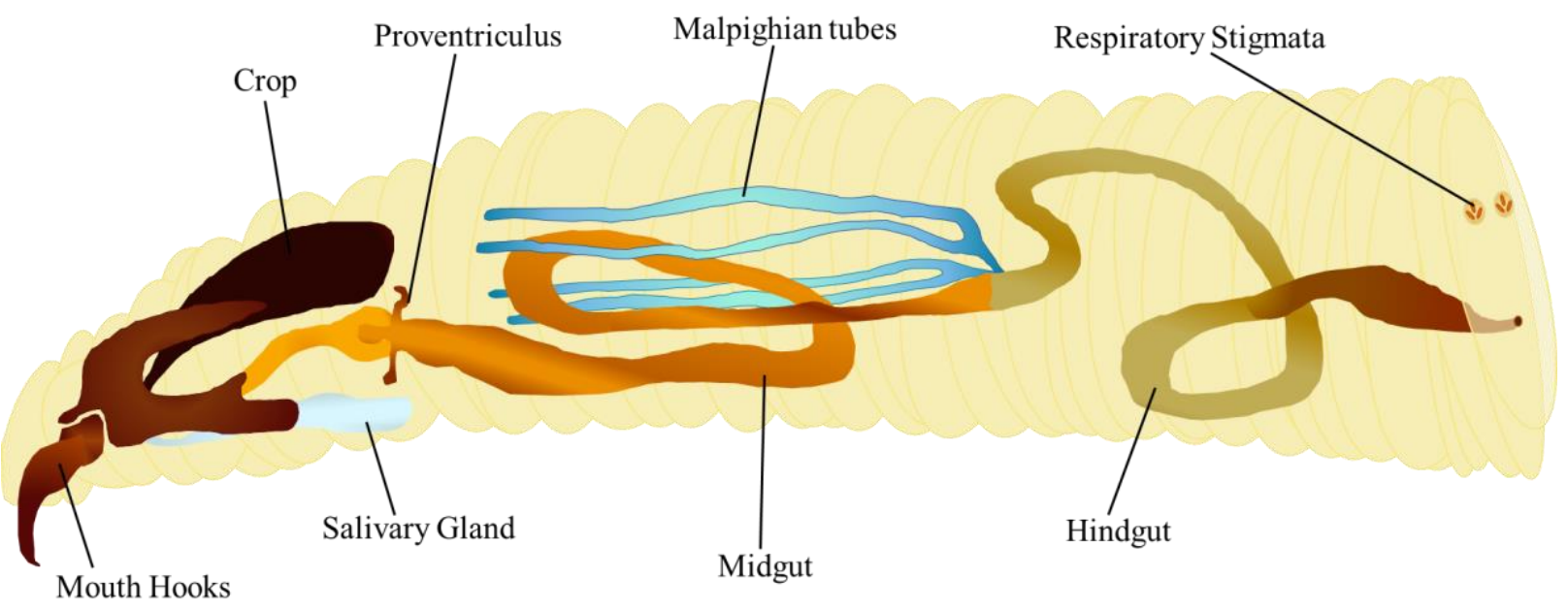

Figure 2.6: Diagram of the alimentary canal of a larval blow fly (25).

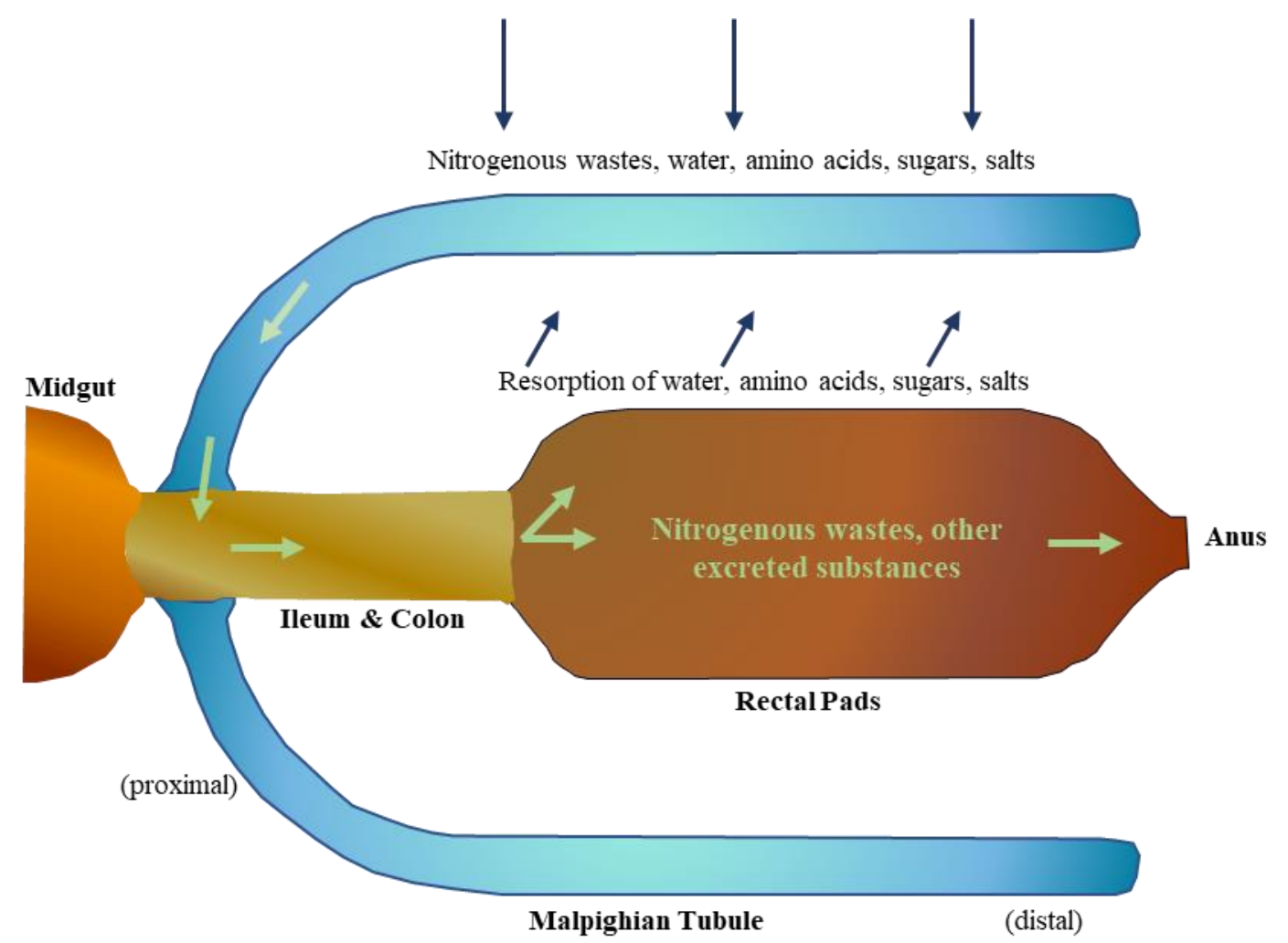

Figure 2.7: Diagram of a Malpighian tubule (29). 


\subsubsection{Insect Age Estimation}

Insects serve an important ecological function as the major driver of mechanical breakdown of human and animal corpses in the natural world. Assuming that the cadaver is accessible to them, insects typically arrive shortly after death, colonize the cadaver with their eggs, and persist through the decomposition process until the cadaver is skeletonized $(30,31)$. As a result of this natural association, forensic entomology techniques can be the most accurate method for estimating the ${ }_{\min } \mathrm{PMI}$ beyond 72 hours after death (32-35). Entomological estimation of $\min P M I$ is rooted in the assumption that insects arrive shortly after death and that their development is primarily governed by temperature (2). To calculate this, two general factors are considered: the time it takes for the necrophagous insects to arrive at the remains and the temperature-dependent rate that is needed for the insects to progress through their life cycles $(8,21,32,33)$. A series of factors are required to be accounted for when employing entomological methods of PMI calculation including the process of colonization, the development time of the insect, decomposition of the corpse, as well as the distribution, biology, ecology, and behavior of the insects $(8,33)$. With the knowledge of the species of the insect, the stage of development, the weather conditions including temperature and humidity, and the growth rate of the insect, a reliable time since death can be determined $(7,22,33)$. Using the relationship of insect development and temperature, the age of an insect can be calculated based on the physical size or stage of the insect and the thermal history at which the insect developed $(2,7,21,22)$. Common approaches for ${ }_{\text {min }} \mathrm{PMI}$ estimation are thermal summation models, curvilinear models, isomorphen, and isomegalen diagrams $(2,22,31,32,36)$. Each of these approaches uses either physical characters of length, width, mass, or current developmental stage of the collected insect to estimate its temporal age, which in turn serves as a proxy for the $\min _{\text {PMI }}(2,22)$. Of these, larval length and developmental stage of the insect are the most utilized technique currently (37). 
Once a corpse is discovered, estimating a minimum time since death is still one of the most fundamental questions to be answered and the application of the developmental rates of necrophagous insects located on the body is crucial for calculating the PMI in these legal cases $(7,21,22)$.

\subsection{Forensic Entomotoxicology}

The term entomotoxicology was first coined in 1994 to describe the combination of entomology, forensic science, and toxicology in regards to the detection of drugs in insects feeding on tissue with drugs or toxins present and the effects caused by these xenobiotics on the insects' life cycles $(22,38-40)$. Depending on the research orientation, we can identify 2 major subclasses: (1) the detection of drugs or toxins in insects after feeding on media with drugs or toxins present and (2) the effects caused by the ingestion of drugs or toxins on the growth and development of the insects $(39,41,42)$.

\subsubsection{Impact on ${ }_{\min } \mathrm{PMI}$}

Even though entomology can be used to estimate the ${ }_{\min } \mathrm{PMI}$ due to the predictable nature of insect growth, the base assumption of predictable growth in a given environment is violated when insects are affected by xenobiotic influences if drugs or poisons present in the cadaver they have colonized (4345). Since 2004, entomotoxicology research has slowly shifted focus from correlation of larvae to blood concentrations and towards drug detection in later life stages or the effect drugs have on the insect growth and development. In general, drugs that are stimulants in humans have been shown to speed up development times for insects and drugs that are depressants have been shown to slow down the development time. For instance, recent reports including methamphetamine, cocaine, ketamine, morphine, and tramadol have shown contrasting effects on insect development (45-49). Stimulant drugs such as methamphetamine and cocaine, appeared to have an increasing effect on the physical development rate and body size of the blow flies $(46,48)$. It was found that the overall developmental time was shorter in the presence of cocaine, and the general insect growth being larger for both cocaine (Chrysomya albiceps (Wiedemann) (Diptera: Calliphoridae) and Chrysomya putoria (Wiedmann) (Diptera: Calliphoridae)) and methamphetamine laced diets (Calliphora stygia (Fabricius) (Diptera: Calliphoridae)) 
$(46,48)$. Among depressant-type drugs, morphine was shown to have no significant effect on size or development rates of $C$. stygia (47); however, ketamine and tramadol appeared to increase body size for the larvae of Lucilia sericata (Meigen) (Diptera: Calliphoridae) $(45,49)$. Changes in physical characters or developmental rates of insects due to the presence of xenobiotics can significantly impact $\min P M I$ estimations, leading to either under- or overestimation of time since death (46). Understanding the effects that drugs can have on insect growth and development is important in terms of estimating $\min ^{\mathrm{PMI}}$ in cases that involve toxins or overdose deaths (50).

Previous case studies have shown that insects feeding directly from tissues containing drugs or illicit substances will incorporate the xenobiotics into the insect's tissue $(22,39)$. When feeding on tissue with drugs present, the effects on insect growth vary by the drug and the concentration $(39,42,51)$. Changes in growth and development of the insect caused by exposure to xenobiotics can create discrepancies in PMI estimations. The effects of heroin on Boettcherisca peregrina (Robineau-Desvoidy ) (Diptera: Sarcophagidae) created PMI discrepancies of 29 hours with similar results for Lucilia sericata at 24 hour discrepancies (51). Cocaine and methamphetamine appeared to accelerate the rate of necrophagous fly development while barbiturates were found to lengthen the larval stage and in turn slow down development into the pupal stage (51). Chrysomya megacephala (Fabricius) (Diptera: Calliphoridae) larvae reared on tissue containing malthalion developed slower than the control group and the time required to emerge as adults from pupa was delayed to 10 days compared to 7 days in the control group (51).

Studies have shown that drugs ingested by the deceased can affect the growth of insects feeding on the body $(39,52)$. Some drugs have suggested to speed up growth, like codeine, cocaine, diazepam, and methamphetamine, while others, tramadol, methadone, methylphenidate, or phenobarbital have indicated to slow the development of forensically relevant insects. Tramadol was reported as increasing the size of larvae but increased the total development time (45). The effect of methadone was limited but 
a high concentration $(4 \mu \mathrm{g} / \mathrm{g})$ the rate of development was decreased (44). Methylphenidate and phenobarbital when present in the media larvae were reared on, increased the total development time by 24 hours and 12 hours respectively (53). Codeine was reported as decreasing the time of larval development to pupa by 24 hours and decreased the time for emergence from pupa to adult by 48 hours (54). Carvalho et al. reported larvae treated with cocaine containing feed stock emerged 60 hours before the non-treated control group (48). Diazepam was reported to decrease the overall development time of Chrysomya putoria by over 90 hours (55). Mullany et al. investigated the effects of methamphetamine on Calliphora stygia and reported a decrease in larval development time of 44 hours and a decrease of pupal emergence to adult time of 78 hours (46). The significance of this data is a PMI calculation can be incorrectly determined based on the size or stage of growth of the insect if drugs are present when the insects are actively feeding (46). From a forensic toxicology perspective, in decomposed corpses suspected of opioid fatality, insect life stages can provide valuable information currently unexplored. Indeed, no entomotoxicological studies have been observed for the extraction and quantitation of fentanyl from insect tissue or the effects of fentanyl on insect growth. As a result, there is a need to assess the value of entomotoxicology on forensic investigations of opioids like fentanyl and analogs.

\subsubsection{Toxicology Specimens}

The succession that occurs on a corpse after death is a relatively confined and closely packed ecosystem that is typically restricted to the remains and proximity. If xenobiotics are present in the body, they will be ingested by the organisms feeding on the corpse. For postmortem toxicological analysis, depending on the stage of decomposition and availability, matrices like blood, urine, liver or heart tissue are often specimens that are analyzed. However, under certain conditions, the stages of decomposition can progress quickly leaving traditional toxicological matrices unavailable $(39,56)$. Fly larvae has been used as a toxicological analysis matrix in these instances $(38,39,57)$. In some cases, larvae matrices can produce less endogenous interferences compared to advanced decomposed tissues (56). Detection of 
drugs in insects can be vital in corroborating traditional toxicology matrices or as the only matrix available in cases with advanced decomposition, exsanguination, or burn victims $(39,58,59)$.

A case where only fly larvae were used to determine drug use was presented by Beyer et al. when a deceased individual was found with no fluids or organs to test for drugs and only fragments of skin present (58). The deceased had been last seen 14 days prior to discovery and the remains were badly decomposed with the head, thorax, and abdomen completely skeletonized (58). The decedent had a history of suicide attempts, a recent prescription for 100 tablets of phenobarbital, and the bottle was found empty in her purse (58). Analysis and detection of phenobarbital in the larva collected from the cadaver was the only toxicology results available for corroborating the physical evidence found on the deceased (58).

In a case presented by Bugelli et al., benzodiazepines and metabolites were detected in the burnt cadavers of a maternal filicide-suicide by fire (59). In this case, the detection of drugs in the insects was used to corroborate the findings of drugs in the fluids and tissues collected (59). When there is a death with a delayed recovery like suicide or drug overdose, advanced decomposition of the body can occur to the point where insect tissue is the only matrix available or can corroborate the findings of any more traditional matrix available (39).

\subsection{Insects of Interest}

Lucilia sericata was chosen as the target species for this study because it is common to many regions of the US (including West Virginia) and the rest of the world. L. sericata is considered as a Holarctic species but has been documented as an invasive species in many parts of the southern hemisphere including Colombia and Australia $(8,60)$. Due to it being a widely distributed blow fly species, Lucilia sericata has also been the most used species in entomotoxicological research with at least 13 papers being published since 1980 (61). The species is a first wave colonizer and reproduces quickly making it a prime choice for in colony decomposition research (21). As one of the first organisms to reach a body after death, 
L. sericata is often collected by entomologist and used in PMI estimations (61). The blow fly L. sericata can be found over a broad range of landscapes and is considered one of the most common blow fly species making it a candidate for many biological and development studies (21). Multiple studies on the development of $L$. sericata have been performed monitoring growth from egg to adult at different temperatures, evaluation of feeding diets, size distributions, developmental plasticity, and testing of PMI estimation models $(8,17,21,23,24,60)$. Of the necrophagous insects studied for entomotoxicology purposes, L. sericata is the most prevalent (61). A desired model species should be associated with forensic casework with a direct relationship with decomposing corpses, be geographically widespread and common or abundant, and their husbandry should be easy enough to facilitate research (61). The blow fly Lucilia sericata fulfills this criteria and was chosen as the target species (61).

\subsection{Establishment of Colony}

Adult colonies of L. sericata were established in May 2018 from field collected specimens from Morgantown, West Virginia. The collected insects were allowed to grow to adulthood and adults were identified as $L$. sericata by physical morphological characters (62). The adult colony at the time of the study was at generation 30 without the addition of new flies to reduce genetic variation within the colony (21). The colony was housed in BugDorm1 (MegaView Co., Ltd., Taiwan) screen cages $(30 \mathrm{~cm} \times 30 \mathrm{~cm} \times 30$ $\mathrm{cm}$ ) stored in a Percival I36LLVLC8 incubator (Percival Scientific, Inc., Perry, lowa, USA) at $25{ }^{\circ} \mathrm{C}\left( \pm 0.5^{\circ} \mathrm{C}\right)$ at $65 \%$ relative humidity ( $\pm 10 \%)$ with a $12: 12$ (L:D) photoperiod. Adults were given honey and water ad libitum and raw beef liver was provided for oviposition. New generations were separated from adults after oviposition to signal the beginning of the next generation and were moved to a new screen cage during emergence. 


\subsection{Postmortem Toxicology}

When a death is sudden or unexpected, investigation into causes or contributing factors generates the need for toxicology testing (63). Interpretation of analytical postmortem toxicology results can be a challenge with often two or more matrices being utilized with preference to peripheral blood and another specimen to corroborate the findings $(63,64)$. Challenges include autolysis, putrefaction, drug degradation, and postmortem redistribution $(63,65)$. Postmortem redistribution (PMR) is described as the passive diffusion of drugs in higher concentrations moving to areas of lower concentration after death (63-66). With lipophilic drugs, the gradient is usually moving from higher concentrations in tissues to the lower concentrations in blood, thereby artificially increasing the blood drug concentration $(63,64)$. A tissue specimen, usually liver, is selected for quantitation in combination to blood specimens to corroborate and adjust for PMR in postmortem testing $(63,64)$. In cases with advanced decomposition or with drugs with higher tendencies for PMR, tissue concentrations can be more reliable for interpretation compared to cavity or decomposed blood specimens (64). Putrefaction and autolysis during decomposition can create challenges for a toxicologist as the sample extraction and clean-up can be affected (63). The need for further sample clean up or different extraction techniques may need to be employed to counteract the effects of decomposed specimens $(1,63,67)$.

\subsection{Extraction Techniques}

Toxicology analysis consists of two main components, the ability to liberate or extract a drug or toxin from a matrix and the instrumental analysis of the extracted drugs. Though these components can be optimized independently, toxicology analysis cannot be successful if one of the components is not effective. Depending on the desired concentration range and the complexity of the sample matrix, extensive sample preparation or extraction can be necessary. The effects of the matrix can cause ionization suppression or enhancement, especially for LC-MS/MS analysis. When trying to quantitate low concentrations, the reduction of interfering endogenous components of the matrix can be crucial. 


\subsubsection{Liquid-Liquid Extraction}

A traditional extraction technique is liquid-liquid extraction (LLE). This technique involves an aqueous phase and an organic solvent phase with a partitioning of the desired compounds to one phase or the other (68). To partition a drug into the organic or aqueous phase, the $\mathrm{pH}$ of the extraction is manipulated to allow for the drug to partition. A style of LLE that uses a miscible solvent is often referred to as dilute and shoot, as the aliquot of the specimen is diluted by the solvent which merely crashes out the proteins of the sample leaving a less than optimal sample (67). Samples extracted by dilute and shoot are often harsher on instrumentation and can lead to more drastic matrix effects, especially in LC-MS/MS analysis (67). The more widely used version of LLE involves an aqueous sample and an immiscible solvent. For extraction of drugs of abuse, the pKa of the target analyte and chemistry are necessary as that will determine how the $\mathrm{pH}$ needs to be manipulated to partition the drugs into the organic or aqueous layers. Typically, the $\mathrm{pH}$ is manipulated to drive the drug into the organic layer of the extraction with a $\mathrm{pH}$ manipulation of lower for acidic drugs and higher for basic drugs. Once the drug partitions to the organic layer, the layer is removed, dried, and reconstituted in a phase suitable for instrumental analysis $(69,70)$. LLE is considered a cheaper extraction process but has disadvantages of harsh organic solvents, a higher volume of organic solvent use, and is generates a generally less clean sample. LLE is often used for blood, urine, and other liquid matrices but has been applied to more complicated matrices such as bile, stomach contents, liver, kidney, and brain tissues $(69,70)$.

\subsubsection{Solid-Phase Extraction}

An often-used extraction technique that provides a more robust sample clean-up is solid-phase extraction (SPE) (71). SPE can be used in analytical procedures for sample clean-up, compound extraction, class fractionization, or for preconcentration for trace analytes $(71,72)$. Compared to traditional LLE procedures, SPE generally uses less organic solvent and removes more co-eluting matrix components (71). In simple terms, SPE consists of a solid phase or sorbent, typically in a cartridge, that has a form of affinity for the target analytes $(71,72)$. Common sorbents include reverse-phase, normal-phase, ion-exchange, 
mixed-mode (ion exchange and reverse-phase), adsorption, size exclusion, magnetic, and immunosorbents (71). SPE procedure fundamentals consist of a preconditioning step, loading of a sample on the solid phase sorbent housed in the cartridge that has an affinity for the target analytes, a wash step to rinse the sample of any unwanted matrix components, and an elution of the target analyses from the sorbent with a solvent selected to overcome the affinity of the target analytes to the sorbent (71). This technique helps remove salts and other components of the matrix, however, components with similar properties as the target analytes will still be coeluted. SPE has been applied to extraction for a wide range of drugs in biological fluid and tissues including blood, urine, oral fluid, bovine milk, and liver tissue (7378).

There are advantages and disadvantages for LLE and SPE in comparison to each other between cost, resource consumption, sample clean-up, and overall extraction ability. LLE is considered a cheaper choice as there is no need for the single use SPE cartridge and does allow for direct extraction of a semisolid matrix however, the use of increased harsh organic solvents and potential for matrix effects by coelution are strong disadvantages. SPE techniques reduce the use of organic solvents while providing a more effective reduction in coeluting matrix components but does increase the cost of sample processing and cannot directly extract from a semi-solid specimen such as tissue homogenate without further sample preparation.

\subsubsection{QuEChERS Extraction}

For pesticide analysis, a quick, easy, cheap, effective, rugged, and safe (QuEChERS) extraction for pesticide analysis in produce samples was presented by Anastassiades et al. (79). Since the application was in agriculture, larger sample sizes of $10 \mathrm{~g}$ were used. A homogenized produce sample was extracted with acetonitrile and a mixture of salts, magnesium sulfate $\left(\mathrm{MgSO}_{4}\right)$ and $\mathrm{NaCl}(79)$. $\mathrm{No} \mathrm{pH}$ adjustment was performed. Acetonitrile (ACN) and water are miscible and the two are not typically chosen for LLE extraction except for dilute and shoot techniques, however, with the addition of salts distinct partitioning 
phases are created much like nonpolar solvents with water (79). The salts will dissolve into any aqueous layer but will not partition into the organic layer, creating distinct layers. Acetonitrile is considered to have a low chronic toxicity compared to nonpolar organic solvents (79). An aliquot of the organic layer was then transferred to a dispersive SPE vial containing primary secondary amine (PSA) sorbent and anhydrous $\mathrm{MgSO}_{4}$ for further sample clean-up (79). The dispersive-SPE acts as a chemical filter to remove coeluted matrix components such as fatty acids, lipids and pigments without the extensive process of traditional SPE (79). QuEChERS can be considered a two-part process with the first part being a LLE with salting out and a second part a SPE clean-up but all within a simplified workflow. Modifications to the protocol, including a reduction of sample and extraction volumes, have been adapted to allow for QuEChERS extractions to be applied to forensic casework (1). QuEChERS extraction protocols have been to forensic biological matrices including blood, urine, and liver tissue (1, 4, 80-82). QuEChERS combines the best parts of traditional LLE and the advantages of traditional SPE without sacrificing selectivity or robustness (80, 83).

\subsection{Drug Overdose Deaths}

Deaths caused by the abuse or poisoning from drugs continues to be a burden on the United States health system with 70,630 drug overdose deaths in 2019 alone (84). The age-adjusted rate for drug overdose deaths in the US is 21.6 per 100,000 which is elevated compared to 20.7 in 2018 (84). A class of drugs that continues to increase in overdose deaths is the opioid class drugs.

The opioids crisis in the United States has a serious impact on our society. According to the National Vital Statistics System (NVSS) in 2019, more than one hundred people died every day of opioid overdose $(84,85)$. Of the 70,630 drug overdose deaths in $2019,49,860$ involved opioids $(70 \%)(84,85)$. Even more concerning is the increase of overdose deaths involving synthetic opioids (e.g. fentanyl, fentanyl analogs, and tramadol), with an increase from 3105 in 2013 to 36,359 in 2019, almost 12 times higher $(84,85)$. Overdose deaths involving synthetic opioids accounted for over half $(51 \%)$ of the total 
drug overdose deaths in $2019(84,85)$. The prevalence of synthetic opioid overdose deaths creates a need for postmortem toxicological analysis and depending on the stage of decomposition and availability, matrices like blood, urine, liver, or heart tissue are often specimens that are analyzed (63).

\subsection{Fentanyl}

Even though the US is experiencing an opioid epidemic currently, opium derivatives have been used for thousands of years (86). Compounds with an affinity for one of the 7-transmembrane G proteincoupled opioid receptors are classified as opioids (87). Opioid receptors can be further separated into four subgroups: $\mathrm{mu}(\mu)$, kappa $(\kappa)$, delta $(\delta)$, and nociceptin (88-90). Naturally occurring compounds that are found in the opium poppy including morphine, codeine, and thebaine are a subset of opioids referred to as opiates (87). Endogenous peptides, endorphins and enkephalins, also bind to these receptors (87). The discovery of meperidine in 1939, led to more research into deriving drugs from this compound (87). This led to the discovery of fentanyl by Dr. Paul Janssen in the late 1950 s $(86,87)$. The improved analgesic and anesthetic effects of fentanyl over opium derived opioids led to further research into the drug class (86). Research was compiled in an attempt to create the most potent narcotic analgesic possible and the structure to activity relationship of narcotic analgesics generated scientific interest in the area $(86,87)$. The compounds discovered, including fentanyl, have strong central nervous system (CNS) action, high potency and rapid brain entry that produces a rapid onset of action $(86,87)$. These same pharmacodynamic and pharmacokinetic properties that are targeted for effectiveness as therapeutic drugs also make them targets for abuse. Fentanyl is a highly effective $\mu$-opioid agonist with a potency of 50-100 times that of morphine that is used clinically as a schedule II drug and, like other opioids, has considerable addictive potential $(91,92)$. Fentanyl has a $\mathrm{pK}_{\mathrm{a}}$ of 8.43 , a high volume of distribution $\left(\mathrm{V}_{\mathrm{d}}\right)$ at $3-8 \mathrm{~L} / \mathrm{kg}$ and is $84 \%$ protein bound at $\mathrm{pH} 7.4(93-95)$. 


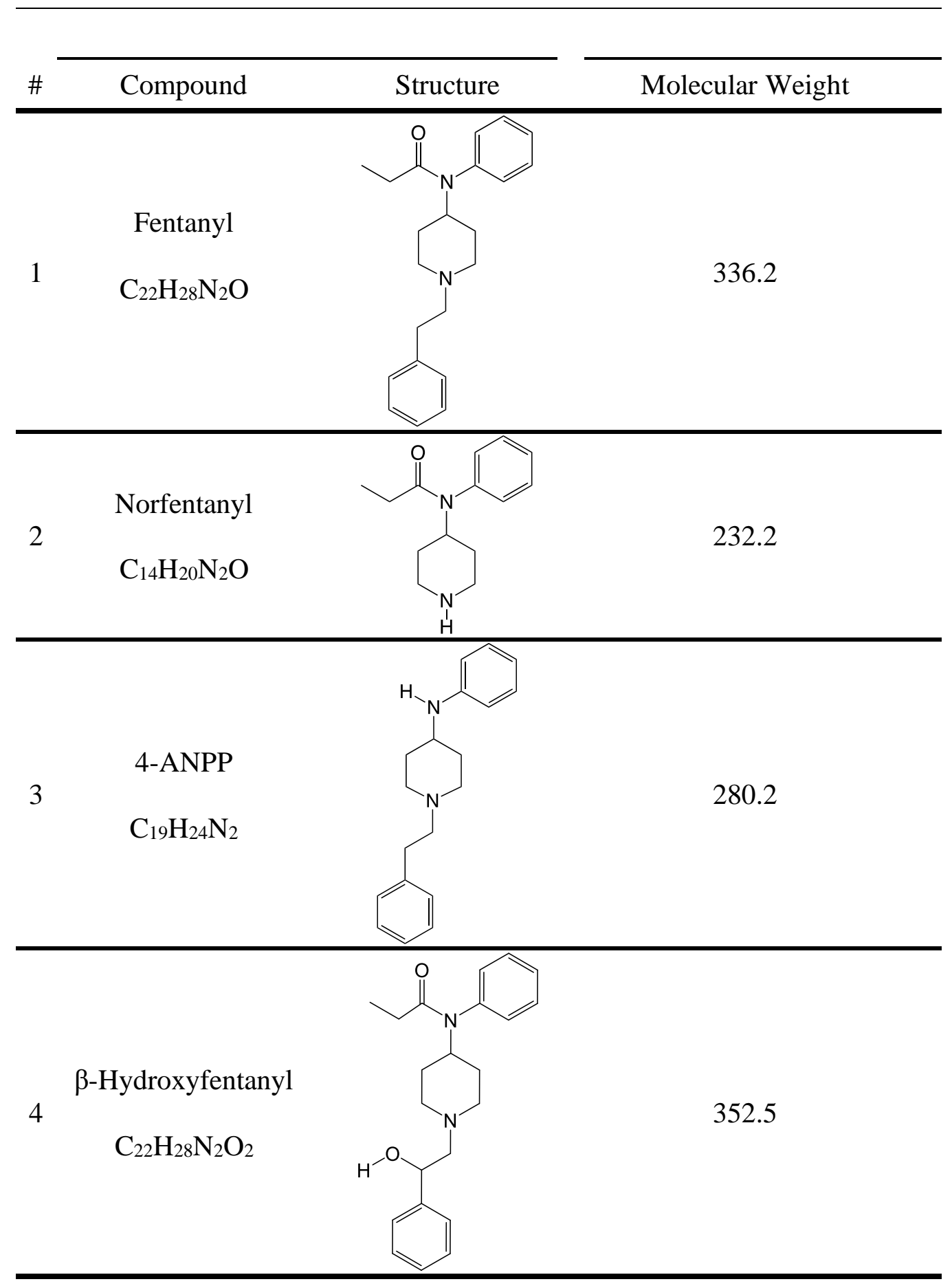


This research from the mid twentieth century lead the ground work for illicit manufacturing of fentanyl and analogs in the early 2010s. Illicitly manufactured fentanyl (IMF) and analogs have been abused individually and combined with other drugs like heroin often in counterfeit pill formulations (9699). The structural scaffold of fentanyl can be modified in numerous ways to generate various versions of novel fentanyl analogs and this is exploited to produce IMF and analogs (Figure 2.6) $(91,92,98)$. Modifications are made to the core fentanyl scaffold in an attempt to avoid legislation or to modify potency $(100,101)$. The four main regions include the amide region, the aniline ring, piperidine ring, and the $\mathrm{N}$-alkyl chain (Figure 2.6). Table 2.1 displays fentanyl and three common metabolites.

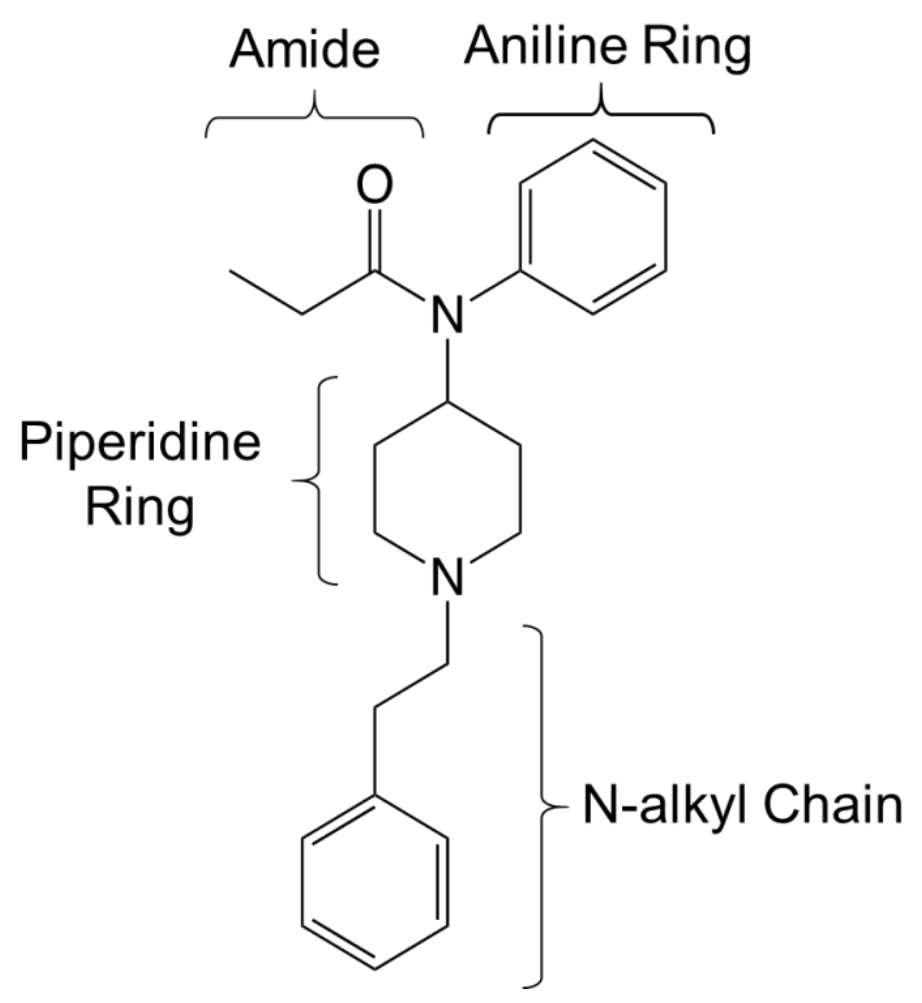

Figure 2.8: Fentanyl scaffold. 


\subsection{Metabolism}

The extensive metabolism of fentanyl in humans has been well documented with most elimination driven by first pass hepatic metabolism (102). Primary metabolites generated by human metabolism of fentanyl are norfentanyl and despropionyl fentanyl (4-ANPP) with norfentanyl being the most abundant (102). Cytochrome P450, specifically CYP3A4, is highlighted as the primary actor in the first pass metabolism for fentanyl in human metabolic pathways $(91,92,102)$. Fentanyl is metabolized in the liver to norfentanyl by CYP3A4 through oxidative N-dealkylation at the piperidine ring (Figure 2.7) (91, 102). $\beta$-hydroxyfentanyl is formed by hydroxylation on the $\mathrm{N}$-alkyl chain (Figure 2.7). Fentanyl is metabolized to 4-ANPP by carboxamide hydrolysis of the amide group (Figure 2.7). In human metabolism, less than $10 \%$ of fentanyl is excreted as the parent drug and the rest are excreted as metabolites (91).

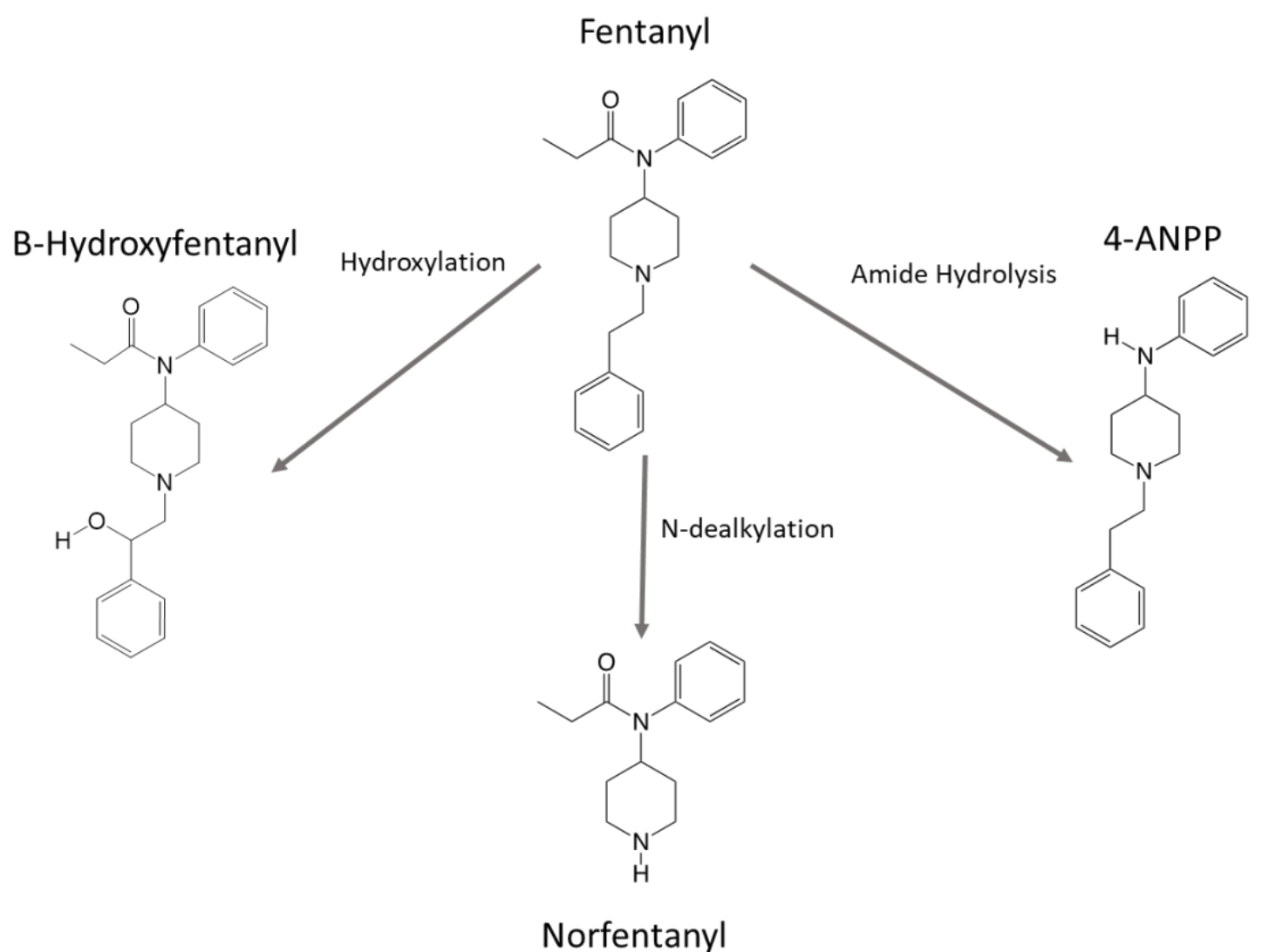

Figure 2.9: Metabolism pathway for major and minor metabolites of fentanyl in humans. 
The excretory system of an insect consists of Malpighian tubules (MT) and the hindgut (Figure 2.5) (29). The Malpighian tubules are responsible for the transport of xenobiotics from within the insect's coelom to the hindgut for excretion (29). In general, water soluble molecules are filtered out of the hemolymph by the MT and hydrophobic molecules would need to be bio transformed (44). The chemistry of P450 enzyme has been exploited to produce bioactivated insecticides where metabolism via the P450 enzyme creates a more toxic compound once metabolized in the insect but these insects were not necrophagous (29).

Metabolism mechanisms of drugs in blow fly species have not been thoroughly investigated and broad metabolic assumptions are limited. For Calliphora stygia, Parry et al. reported when maintained on meat containing morphine, the insects only contained morphine with no substantial metabolites detected (28). Excretion reported as clearance rate of morphine was reported indicating even without significant metabolism for the drug in the insect, elimination of the drug was still possible (28). Norcodeine and morphine were detected in L. sericata raised on a diet of pig liver fortified with codeine (103). This could be evidence of insect metabolism but toxicology analysis of the liver tissue detected norcodeine and morphine so metabolism of the liver enzymes could not be ruled out as the source of the metabolites (103). Gosselin et al. reared L. sericata on a diet of beef hearts fortified with methadone and were able to detect EDDP in some of the high treatment specimens (44). The authors presented this data as evidence that the insects biotransformed the methadone to EDDP to better excrete the drugs and the observation of methadone and EDDP in only the high treatment specimens is further indication of the insects ability to excrete the more hydrophobic metabolite (44).

\subsection{Validation}

Toxicology method validation guidelines from the Scientific Working Group for Forensic Toxicology (SWGTOX) were replaced by the American Academy of Forensic Sciences (AAFS) Academy Standards Board (ASB) 036 requirements $(104,105)$. Method validations were performed using this 
standard for the model and the evaluated parameters included calibration model, interference studies, ionization suppression/enhancement, dilution integrity, processed sample stability, bias, precision, limits of quantitation and detection (Figure 2.8).

\subsubsection{Calibration model}

For each validation presented in the subsequent chapters, calibration curves were evaluated using at a minimum of six non-zero concentrations with a minimum of five replicates. This calibration curves represented the analytical measurement range (AMR) for each target analyte. Calibration curves were analyzed weighted $(1 / x)$ in MassHunter. These curves were performed in matrix matched extractions and were performed simultaneously with the bias and precision extractions. Residual plots were examined for constant variance. It is crucial to show that the calibration curve is linear throughout the curve for accurate calculation of concentration. All of the ASB standard 036 procedures were followed unless stated otherwise.

\subsubsection{Interference studies}

To evaluate interferences, the impact of any inference of the target analytes to the internal standards, any interference of the internal standard to the target analytes, any interference of the matrix to the target or internal standards, and any interference from commonly encountered analytes were considered.

To evaluate interferences a high concentration (highest calibrator) of the target analytes was injected with no IS to show any interference of the drug standards to the IS. Conversely, an aliquot of IS with no target analytes was injected to observe any interference caused by the IS. Matrix interferences were evaluated by extracting blank matrix sources $(n=10)$ for the matrix of interest with no IS added. To evaluate potential interferences from commonly encountered compounds, a mix of common drugs encountered in toxicology was injected. 


\subsubsection{Ionization suppression/enhancement}

Ionization suppression or enhancement was evaluated at two concentrations, low and high, for ten replicates at both concentrations. To be deemed acceptable, the percent ionization suppression or enhancement shall not exceed $\pm 25 \%$ with a percent relative standard deviation $(\% C V)$ not exceeding $20 \%$ (104). A post-extraction addition approach was utilized by comparing neat standards (Set 1) to blank matrix samples fortified with neat standard after extraction (Set 2). Set 1 consisted of neat standards prepared at low ( 2 times the LLOQ) and high ( $50 \%$ of the highest calibrator) and were injected ten times to establish the mean peak area for each target analyte. Set 2 consisted of ten individual blank matrix sources in duplicate, for low $(n=10)$ and high $(n=10)$ concentration, extracted and spiked with the low and high concentration, respectively, after extraction. The average area of each set $(\bar{X})$ was used to calculate the suppression or enhancement effects at each concentration for each analyte is as follows:

$$
\text { Ionization suppression or enhancement }(\%)=\left(\frac{\bar{X} \text { Area of set } 2}{\bar{X} \text { Area of set } 1}-1\right) * 100
$$

\subsubsection{Bias and Precision}

For bias and precision calculations, blank tissue samples were fortified and analyzed at low, medium, and high concentrations for the target analytes in triplicate over five days $(n=15)$. Bias was calculated as a percent deviation of the extracted mean concentration from the theoretical concentration with an acceptable bias not exceeding $\pm 20 \%$. Precision was evaluated by percent standard deviation (\%CV) of the mean at each concentration with the criteria of not exceeding $20 \%$.

\subsubsection{Limits}

For the evaluation of the limit of detection (LOD) and the lower limit of quantitation (LLOQ), blank matrix sources for each tissue were spiked with concentrations at the lowest non-zero calibrator. 
Triplicates of each blank matrix sources were spiked at the lowest non-zero calibrator and analyzed over three days $(n=9)$. The acceptable parameters were bias $( \pm 20 \%)$ and precision $(\% C V \leq 20 \%)$. For LOD, chromatographic acceptance was evaluated and deemed acceptable when signal to noise $(\mathrm{s} / \mathrm{n})$ ratios were above 3.3 and qualifier ratios did not exceed $\pm 20 \%$.

\subsubsection{Carryover and Dilution Integrity}

Blank matrix samples were injected directly after injections of samples three times greater than the highest calibrator in triplicate analysis to evaluate carryover. Carryover was not considered significant if present below $10 \%$ of the lowest calibrator (LLOQ). For dilution integrity, blank matrix samples from both sources were spiked at concentrations three times the highest calibrator in triplicate and extracted simultaneously with blank matrix only spiked with internal standard in triplicate. The resulting extract was diluted 1:5 (S:T) with the extracted blank matrix with internal standard $(5,106)$. The process was repeated over a period of five runs $(n=15)$ and the dilution integrity was deemed acceptable if bias did not exceed $\pm 20 \%$ and the precision (\%CV) did not exceed $20 \%$.

\subsubsection{Processed Sample Stability}

To determine the stability of processed samples for each tissue type in the autosampler $\left(25^{\circ} \mathrm{C}\right)$, blank matrix samples were fortified at low and high concentrations and extracted. Post extraction reconstituted samples, for low and high respectively, were pooled and aliquoted to LC-MS/MS vials. Triplicate analysis at time frames: $0,12,24,36,48,60$, and $72 \mathrm{hr}$ were performed for each concentration. Average peak area was calculated for each triplicate and compared to time zero. An acceptable bias threshold was set at $\pm 20 \%$.

\subsubsection{Recovery and Process Efficiency}

To calculate recovery of the extraction (RE) and process efficiency (PE) two formulas were utilized.

Variables in the formulas represent the mean peak areas for the neat solution (A), the mean peak areas for the standards spiked after extraction (B), and the mean peak areas for the standards spiked before extraction (C) (107). Recovery and process efficiency were at low and high concentrations for ten 
replicates $(n=10)$ for each matrix type. Ideally, a perfect recovery or process efficiency would be $100 \%$ and $\pm 20 \%$ of this value is desired while not exceeding a \%CV of $20 \%$. The ASB Standard 036 does not have a requirement for recovery or process efficiency and the $\pm 20 \%$ while not exceeding a $\% C V$ of $20 \%$ is a selfimposed guideline.

$$
\begin{aligned}
& R E(\%)=\frac{C}{B} * 100 \\
& P E(\%)=\frac{C}{A} * 100
\end{aligned}
$$




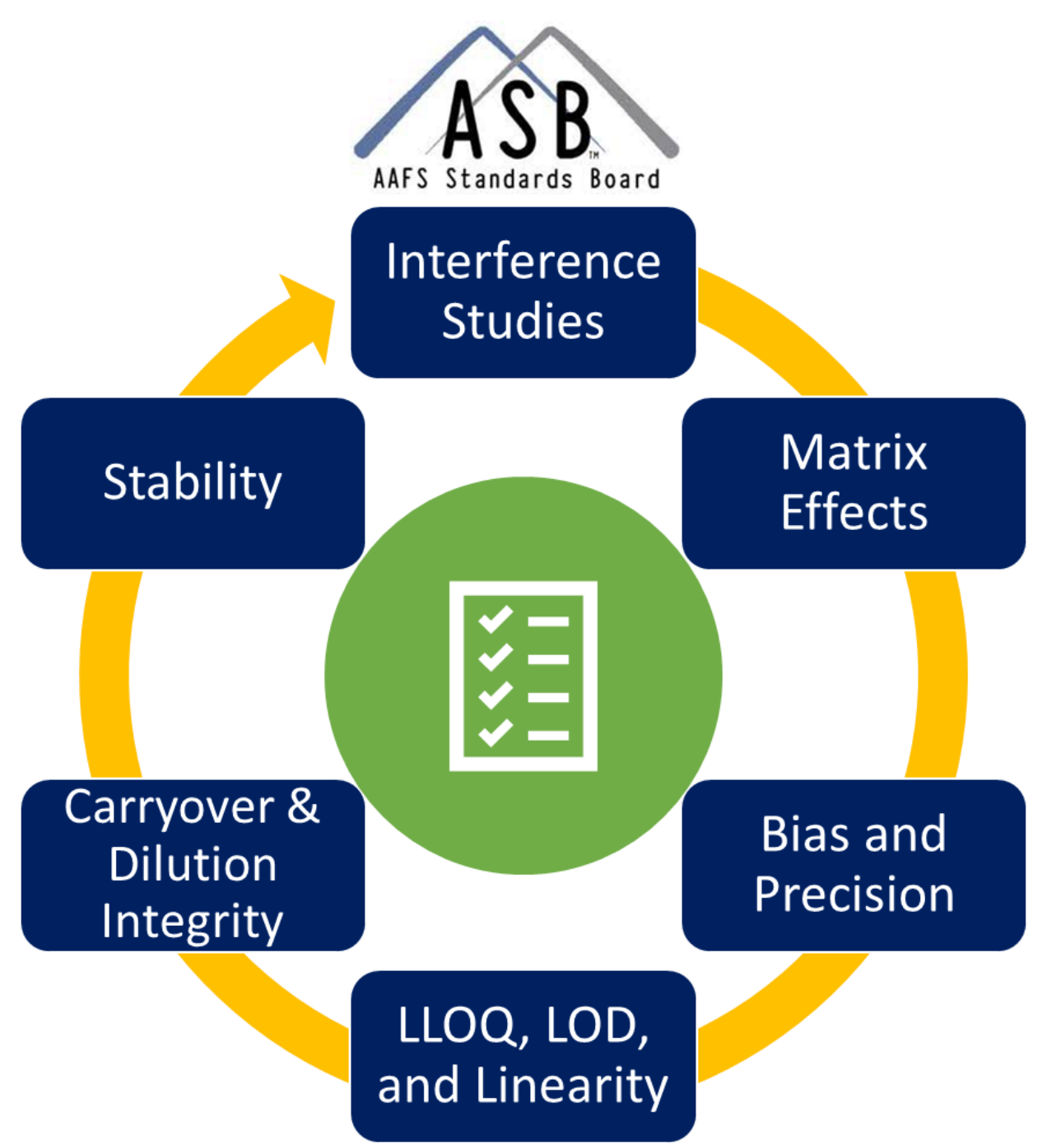

Figure 2.10: Overall design for validations following the ASB Standard 036.

\subsection{Critiques and Current Challenges}

In previous studies, toxicology correlations from insect concentrations to blood or liver concentrations has been limited due to large standard deviations in measurements. Tracqui et al. found large inter-larvae variations for meprobamate and nordiazepam as well as variations in inter-site pooled larvae samples from 5 body locations for nordiazepam and cyamemazine (108). The anatomic sites monitored were upper digestive tract, perioral area, trunk, upper limbs and lower limbs with concentrations ranging from $24 \mathrm{ng} / \mathrm{g}(\mathrm{ppb})$ to $837 \mathrm{ng} / \mathrm{g}(\mathrm{ppb})(108)$. While these numbers are concerning, 
no indication was given for what stage the maggots sampled were in (first, second, third, or wandering) or if the maggots sampled were from a single species or multiple species. Variations in life stages, especially if oviposition occurred at a separate time, or if multiple species were sampled can contribute to deviations in measured concentrations. A feeding third instar has the potential for drug infused tissue to be present in the crop of the maggot that has yet to be incorporated in the insect tissue whereas a wandering third instar are identified as having an empty crop and drug concentrations detected would be from the insect tissue (46). This is important due to drug concentrations in the undigested food material in the crop can create variations in the amount of drug extracted from the insects if feeding third instars and wandering third instars were compared (46). Mullany et al. controlled for this variable by having the feeding insects starved for 4 hours before culling (46). Tracqui et al. provided no indication of stage or species that could be attributed to the measured deviations. No reference tissue concentrations were measured at the 5 different locations that the larvae were pooled from. Williams and Pounder identified that there is a site to site variability of drug concentrations in skeletal muscle (109). Without a reference tissue sample from the sites of collection, it is unknown if the deviations in concentration are to be attributed to the nature of the insect activity or normal site to site variation of skeletal muscle. Tracqui et al. made a bold statement that the drugs detected in maggots can also be detected in the tissue as well, however that is based on the assumption that tissue will be available for analysis (108). In warm arid conditions it is possible for a corpse to desiccate leaving no soft tissue for analysis even if it is a rare occurrence. Tracqui et al. also focused on the larvae and did not address the toxicological value of later insect stages including pupa, adults and empty puparium that can persist longer than both tissue and maggots. 


\subsection{Critical Need}

It is well documented that drugs and toxins in the media insects feed on affects their growth and development, however the effects vary by the class of drug or toxicant, and the concentration $(39,42$, 51). Wallace highlighted differences in rates of elimination, bioaccumulation, and pharmacokinetic characteristics vary between arthropods and between drug classes; pointing to the need for more entomotoxicological experiments to investigate these parameters $(39,110)$. Differences in drug concentrations observed for feeding larvae and post feeding or wandering larvae as well as the later stages of development (111). This suggest that there are differences in metabolism, bioaccumulation, and elimination of the drug as it progresses through its life stages, not only variations caused by differences in drug classes (111). Alterations in insect development caused by toxicants in the feeding material, also alter the calculation of the postmortem interval $(39,110)$. The opioid epidemic has caused a sharp increase in opioid deaths in the United States and observing the effects that opioids can have on insect growth can provide useful information in calculating PMI in opioid related deaths. A review by Da Silva identified 122 toxicants investigated by 63 entomotoxicology publications with none monitoring fentanyl or its metabolites (61). As of this study, there have been no published entomotoxicology journal articles with fentanyl or its metabolites as the drug of interest. With fentanyl abuse increasing along with overdose rates, there is a critical need for evaluating the effects of fentanyl on the growth and development of forensically relevant blow flies.

Recent studies have demonstrated the possibility of correlating concentrations of drugs extracted from insects compared to concentrations of the feeding substrate, however interpretation of results has remained a controversial issue $(39,111)$. Estimation to the cause of death or relationships of blood or liver tissue concentrations to drug concentrations recovered from insects feeding on a corpse remain unachieved and are subject to debate $(39,111)$. Identifying this quantitative relationship is difficult due to complicated influencing factors (111). Variations in insect concentrations are affected by drug tropism in the body, postmortem redistribution, and the extraction and detection efficiencies of the analytical 
techniques $(39,61)$. Sallawad et al. highlighted this need and lack of valuable research into the factors limiting the assessment of correlating insect drug concentrations to tissue drug concentrations with the conclusion that further research into entomotoxicology is necessary (42). The first step in achieving correlation between blood/tissue and insect concentrations is to control or reduce the influencing factors by identifying correlations from insect drug concentrations to single tissue type source drug concentrations. To address this critical need, this study will evaluate liver tissue fortified with fentanyl as the feeding media for the insects in this study. Concentrations were compared to determine any potential correlation. 
Chapter 3: Development and Application of QuEChERS Extraction Method for the Quantitation of Fentanyl and Metabolites from Liver Tissue 


\subsection{Section 1: Method Development and Application}

Reproduced in part with permission from Cox, J., Train, A., Field, A., Ott, C., DelTondo, J., Kraner, J., et al, Journal of Analytical Toxicology, https://doi.org/10.1093/jat/bkaa006.

\subsubsection{Introduction}

In a forensic postmortem toxicological workflow, often various specimens are submitted for testing including: urine, blood, liver, kidney, and other tissues (1). For medico-legal death investigations, the interpretation of toxicological findings is a key component to establish the cause of death of an individual (64). Due to its relevance and legal implications, the accurate measurement of drug concentrations found in a forensic tissue sample is of utmost importance for forensic toxicologists (63). Therefore, significant efforts are devoted to the development of analytical routes to efficiently extract and isolate the target analytes, while simplifying the overall analytical scheme. When working with biological specimens, the analyst is prone to deal with tissues that are in various stages of decomposition. Natural processes like autolysis and putrefaction complicates the tissue sample integrity, this creates a complex challenge for toxicologists.

To overcome the complexity of the matrix, a toxicologist relies on extraction strategies that help to mitigate such challenges. Several outcomes are expected when performing a sample pretreatment: first, the isolation of the analytes of interest from the matrix; second, the removal of proteins, lipids, salts, and other interferent compounds that could obscure the analytical signal of interest and preconcentration of the drugs $(67,112)$.

Often, overdoses occur when fentanyl is unknowingly ingested due to a mixture of drugs or fentanyl being distributed as another drug causing the user to be unaware of the potency of the drug they are consuming, partly attributed to the introduction of illicitly manufactured fentanyl (IMF) into the heroin trade $(99,113)$. Mixtures of illicit drugs such as heroin, cocaine and methamphetamine with IMF increases the risk of overdose due to the user being exposed to varying potency of the product consumed, potentially delivering an exponentially greater dose (113). Unintentional consumption of more potent 
IMF containing mixtures can cause severe unintended consequences including overdose and death. Specifically, between July 2016 and December 2016, 50\% of the opioid related overdose deaths in West Virginia involved fentanyl (113). Analysis of synthetic opioids pose an analytical challenge due to the acute lethal intoxication concentrations, continuous variations of analogs, and wide variations in chemical structure (114).

Many sources provide references range information to aid in the interpretation of fentanyl blood concentrations. However, numerous factors must be given consideration to estimate the likely effects of a specific fentanyl concentration. The fentanyl blood concentration that results in a fatal overdose can vary greatly from person to person depending on factors including but not limited to: age, body fat percentage, fentanyl or opioid tolerance, alcohol consumption, liver health, and other drugs present including prescribed medications $(115,116)$. As postmortem redistribution (PMR) occurs, blood drawn from central cavities often have higher concentrations than blood drawn from peripheral area and is attributed to the diffusion of drug from organs from surrounding tissues $(117,118)$. Fentanyl has a high volume of distribution $\left(V_{d}\right)$ at 3-8 L/kg and compounds with a large $V_{d}$ are more prone to postmortem redistribution $(93,94)$. Olson et al. presented findings of significant PMR for fentanyl when comparing two blood draws; first close to the time of death (between 2.5 and $6 \mathrm{hr}$ ) followed by a second draw (between 7 and $53 \mathrm{hr}$ ) with a mean first draw concentration of $4.6 \mu \mathrm{g} / \mathrm{L}$ and a mean second draw of $17.3 \mu \mathrm{g} / \mathrm{L}(66)$. Olson et al. also reported multiple first draw concentrations of none detected followed by positive concentrations in the second draw in four cases (66). Based on the PMR tendencies of fentanyl, liver or other organ tissue are needed to provide a medical examiner the proper data to make an informed decision on the manner or cause of death when fentanyl is present and may provide a more adequate indication of antemortem concentrations compared to blood (64).

In the body, fentanyl is metabolized mostly via hepatic cytochrome P450 (CYP450), primarily CYP3A4, with norfentanyl and despropionyl fentanyl (4-ANPP) being the major and minor metabolites 
respectively $(91,102,119)$. CYP3A4 has been reported to have metabolic pathways for multiple drugs in the opioid and benzodiazepine classes (119). For investigations involving drug toxicity, medical examiners often evaluate both parent and metabolite concentrations to improve the interpretation of the analytical results $(119,120)$. The ratio of parent drug to metabolite can be useful in determining manner of death, differentiate chronic abuse from acute overdose, misuse versus therapeutic usage or delayed death (119, 120).

Within the drug classes encountered in casework, opioids and specifically synthetic opioids are of particular interest to current practitioners due to increase and prevalence of fentanyl overdoses. Some of the sample preparation schemes for liver extraction in use in forensic toxicology labs include simple procedures like dilute and shoot, protein precipitation, liquid-liquid extraction (LLE) and solid phase extraction (SPE) (67). SPE methods often utilize some form of sample pretreatment to release the drugs from the matrix and to allow the samples to pass through the SPE cartridges (75). Crum et al. and Shoff et al. have presented SPE methods for synthetic opioids in blood and liver tissue $(73,121)$. Coopman et al., Rojkiewicz et al., and McIntyre et al. presented LLE methods for the quantification of synthetic opioids in blood and tissues including liver in 2016 and 2017 (69, 70, 122).

By its nature, the liver contains many lipids that can contribute to matrix effects and be problematic for instrumentation (1). In 2003, Anastassiades et al. presented a methodology for a quick, easy, cheap, effective, rugged, and safe (QuEChERS) extraction for pesticide analysis in produce samples (79). QuEChERS is ideal for tissue extractions due to its two-part process that allows for a direct extraction with organic solvent in the first part followed by a dispersive-solid phase extraction step (81). QuEChERS embraces the advantages of LLE, direct extraction from tissue with an organic solvent and cost effectiveness, combined with the advantages of SPE, reduction of matrix effects and impurities, without sacrificing selectivity or robustness $(80,83)$. Usui et al. demonstrated that a QuEChERS protocol could be modified to test human liver tissue for benzodiazepines (1). A QuEChERS extraction was utilized to extract 
acetyl fentanyl from urine in a fatal poisoning and presented here is an application for a QuEChERS extraction for the quantification of fentanyl in liver tissue (82).

The development and validation of a QuEChERS extraction protocol is presented in this study as an alternative analytical method for efficient extraction and detection of fentanyl and its major metabolites: norfentanyl and despropionyl fentanyl (4-ANPP).

\subsubsection{Methods}

\subsubsection{Chemicals and Materials}

Drug standards: fentanyl, norfentanyl, 4-ANPP, fentanyl-D5, and norfenanyl-D5 were obtained from Cerilliant (Round Rock, Tx) at concentrations of $1 \mathrm{mg} / \mathrm{mL}$ or $100 \mu \mathrm{g} / \mathrm{mL}$ in methanol. Optima ${ }^{\circledR}$ LC-MS grade methanol, acetonitrile, methyl-tertbutyl ether (MTBE), formic acid, and ammonium formate were purchased from Fisher Scientific (Fair Lawn, NJ). Analytical grade hydrochloric acid ( $\mathrm{HCl}$ ) was obtained from Fisher Scientific (Fair Lawn, NJ). Analytical grade sodium hydroxide $(\mathrm{NaOH})$ was purchased from Sigma-Aldrich (St. Louis, MO). All water was purified using a Direct-Q 3 UV Water Purification System (Darmstadt, Germany). Cartridges for SPE analysis were Bond Elut Certify (130 mg; $3 \mathrm{~mL}$ ) purchased from Agilent Technologies (Santa Clara, CA). The QuEChERS original extraction salt packets, containing $6 \mathrm{~g}$ magnesium sulfate and $1.5 \mathrm{~g}$ sodium chloride, and $2 \mathrm{~mL}$ dispersive-SPE tubes, containing $25 \mathrm{mg}$ of primary secondary amine (PSA), $25 \mathrm{mg}$ end-capped octadecylsilane $\left(\mathrm{C}_{18 \mathrm{Ec}}\right)$, and $150 \mathrm{mg}$ magnesium sulfate, were purchased from Agilent Technologies (Santa Clara, CA). A Mixer Mill MM 200 was utilized for homogenization was obtained from Retsch (Haan, Germany). Steel zinc plated BB balls (0.177 cal, 4.5 $\mathrm{mm}$ ) were obtained from Daisy (Rogers, AR).

Pooled liver specimens used in method development and validation were generated from six packages of beef liver purchased from a local retailer packaged by Rumba Meats (Del Rio, TX). Quantitative analysis was performed on authentic human liver specimens submitted from suspected fentanyl-related deaths investigated by the Office of the Chief Medical Examiner Office (OCME) in Charleston, West Virginia. All samples were stored at $-20^{\circ} \mathrm{C}$ prior to analysis. 


\subsubsection{LC-MS/MS}

The quantification method was performed on an Agilent 6470 Triple Quad LC-MS/MS system coupled with an Agilent 1290 Infinity II LC system. Chromatographic separation was achieved on a Zorbax Eclipse Plus C18 RRHD 3.0x100 mm, $1.8 \mu \mathrm{m}$ column with $0.1 \%$ formic acid and $5 \mathrm{mM}$ ammonium formate in water (mobile phase $\mathrm{A}$ ) and $0.1 \%$ formic acid in methanol (mobile phase B). Data acquisition was performed in multiple reaction monitoring (MRM) mode with positive ESI with a principal MRM transition for quantitation and a minimum of one qualifier MRM transition for each analyte. A gradient elution at a flow rate of $0.5 \mathrm{~mL} / \mathrm{min}$ was run as follows: initial hold for $1 \mathrm{~min}$ at $5 \%$ mobile phase $\mathrm{B}$ with a ramp to $40 \%$ mobile phase B for $1 \mathrm{~min}$, followed by a ramp to $80 \% \mathrm{~B}$ in 3 mins with a hold of $30 \mathrm{~s}$, and finally a $2.5 \mathrm{~min}$ post run for re-equilibrium at $5 \% \mathrm{~B}$.

The MS source parameters were performed as follows: gas temperature $325^{\circ} \mathrm{C}$; gas flow $10 \mathrm{~L} / \mathrm{min}$; nebulizer 45 psi; sheath gas flow $11 \mathrm{~L} / \mathrm{min}$; capillary voltage $3500 \mathrm{~V}$; and a charging voltage $500 \mathrm{~V}$. Optimization of the data acquisition parameters was performed utilizing Agilent MassHunter Optimization software in MRM mode to select the optimal precursor ions with corresponding fragmentor voltage and selection of product ions with associated collision energies for each transition. The optimized parameters are listed in Table 3.1.

The quantitation of analytes was performed using Agilent MassHunter Quantitative software version B.08.00. Relative responses were calculated using the absolute response of the drug in relation to response of the IS and plotted against drug concentrations.

The LC-MS/MS analysis was validated following the American Academy of Forensic Sciences Standards Board (ASB) standard 036 requirements (104). Evaluated parameter include selectivity, matrix effects, linearity, processed sample stability, bias, and precision. 
Table 3.1: MRM MS method parameters.

\begin{tabular}{|c|c|c|c|c|c|}
\hline Compound & Transition $^{\mathrm{a}}$ & Fragmentor $(\mathrm{V})$ & $\mathrm{CE}(\mathrm{V})$ & Cell Acc (V) & Internal Standard \\
\hline \multirow{3}{*}{ Fentanyl } & $337.2 \rightarrow 188.0$ & & 24 & \multirow{3}{*}{4} & \multirow{3}{*}{ D5-Fentanyl } \\
\hline & $337.2 \rightarrow 105.0$ & 146 & 48 & & \\
\hline & $337.2 \rightarrow 77.0$ & & 100 & & \\
\hline \multirow{3}{*}{ 4-ANPP } & $281.2 \rightarrow 188.0$ & & 16 & \multirow{3}{*}{4} & \multirow{3}{*}{ D5-Fentanyl } \\
\hline & $281.2 \rightarrow 105.0$ & 122 & 36 & & \\
\hline & $281.2 \rightarrow 77.0$ & & 84 & & \\
\hline \multirow{2}{*}{ Norfentanyl } & $233.2 \rightarrow 84.0$ & \multirow{2}{*}{117} & 20 & \multirow{2}{*}{4} & \multirow{2}{*}{ D5-Norfentanyl } \\
\hline & $233.2 \rightarrow 55.1$ & & 44 & & \\
\hline \multirow{2}{*}{ D5-Fentanyl } & $342.3 \rightarrow 188.0$ & \multirow{2}{*}{141} & 24 & \multirow{2}{*}{4} & \multirow{2}{*}{-} \\
\hline & $342.3 \rightarrow 105.0$ & & 48 & & \\
\hline \multirow{2}{*}{ D5-Norfentanyl } & $238.2 \rightarrow 84.0$ & \multirow{2}{*}{107} & 20 & \multirow{2}{*}{4} & \multirow{2}{*}{-} \\
\hline & $238.2 \rightarrow 55.1$ & & 48 & & \\
\hline
\end{tabular}

Individual stock solutions of each analyte standard were prepared in methanol and stored at $20^{\circ} \mathrm{C}$. An intermediate working solution (IWS) mix of fentanyl $(10 \mathrm{ng} / \mu \mathrm{L})$, norfentanyl $(10 \mathrm{ng} / \mu \mathrm{L})$, and 4ANPP $(8 \mathrm{ng} / \mu \mathrm{L})$. Using the IWS, a serial dilution was performed to prepare stock solutions at concentrations of $0.02,0.04,0.1,0.4,2$, and $4 \mathrm{ng} / \mu \mathrm{L}(\mathrm{ppm})$ for fentanyl and norfentanyl with concentrations of $0.016,0.032,0.08,0.32,1.6$, and $3.2 \mathrm{ng} / \mu \mathrm{L}(\mathrm{ppm})$ for 4-ANPP. An internal standard solution with fentanyl-D5 and norfentanyl-D5 was prepared at a concentration of $100 \mathrm{ng} / \mathrm{mL}$. Separate solutions were made of fentanyl (10 ng/ $\mu \mathrm{L})$, norfentanyl $(10 \mathrm{ng} / \mu \mathrm{L})$, and 4-ANPP $(8 \mathrm{ng} / \mu \mathrm{L})$ for further dilutions for use in bias and precision samples. All solutions were prepared in methanol and stored at $20^{\circ} \mathrm{C}$. 


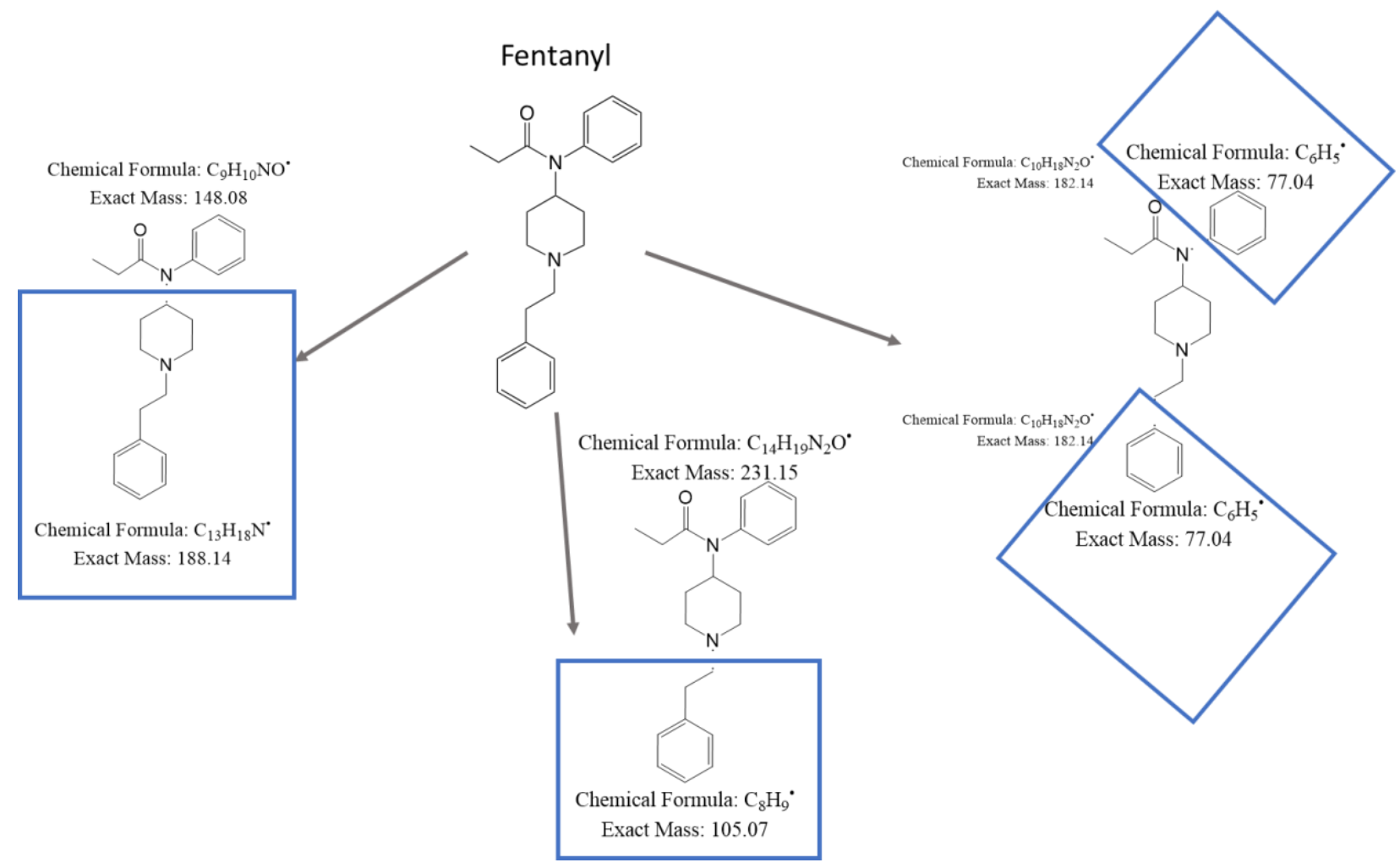

Figure 3.1: Fragmentation predictions from ChemDraw for fentanyl quant transition (left) and the qualifier transitions.

Pooled beef liver specimens, as described above, were aliquoted out in $0.2 \mathrm{~g}$ portions and spiked with $5 \mu \mathrm{L}$ of $0.02,0.04,0.4$, and $2 \mathrm{ng} / \mu \mathrm{L}$ for fentanyl and norfentanyl with corresponding concentrations of $0.016,0.032,0.32$, and $1.6 \mathrm{ng} / \mu \mathrm{L}(\mathrm{ppm})$ for 4-ANPP to create four concentrations of $0.5 \mu \mathrm{g} / \mathrm{kg}$ (LLOQ), $1.0 \mu \mathrm{g} / \mathrm{kg}$ (LOW), $10 \mu \mathrm{g} / \mathrm{kg}$ (MED), and $50 \mu \mathrm{g} / \mathrm{kg}$ (HIGH) for fentanyl and norfentanyl with corresponding concentrations of $0.4 \mu \mathrm{g} / \mathrm{kg}$ (LLOQ), $0.8 \mu \mathrm{g} / \mathrm{kg}$ (LOW), $8 \mu \mathrm{g} / \mathrm{kg}$ (MED), and $40 \mu \mathrm{g} / \mathrm{kg}$ (HIGH) for 4-ANPP. QC samples were created on the day of analysis. 


\subsubsection{Extraction Techniques}

\subsection{QuEChERS}

The modified method proposed reduces the amount of sample required for extraction to $0.2 \mathrm{~g}$ while reducing the risk of cross contamination by homogenizing the sample in a single reaction tube (1). The apparatus utilized steel beads and a bead beater type homogenizer allowing for homogenization of tissue while avoiding cross contamination risks associated with bladed devices (1). This process reduced the amount of QuEChERS materials required for the extraction while reducing the pretreatment process. Drug extraction from tissue requires a method that is sensitive, reduces the matrix effects, and allows for direct extraction from the tissue. Liver tissue is often fatty in nature with many lipids. A modified QuEChERS protocol meets all the requirements for liver extraction and reduces the risk of cross contamination in the homogenization process. A reaction chamber for the mixer mill was created (Figure 3.2) to house the extraction microcentrifuge tubes.

Tissue samples weighing $0.2 \mathrm{~g}$ were placed in a $1.5 \mathrm{~mL}$ centrifuge tube, followed by $400 \mu \mathrm{L}$ deionized water, $100 \mu \mathrm{L} \mathrm{IS}$, and $700 \mu \mathrm{L}$ acetonitrile addition to the tube, along with 3 steel beads. The tubes were vortexed, and $0.2 \mathrm{~g}$ QuEChERS original extraction salt was added to the tubes. The tubes were modified for homogenization by cutting off the lid hinge and tip, then parafilm was wrapped around the top of the tubes to avoid leakage. The samples were then vortexed, mixed with a mixer mill for 1 minute, centrifuged for 5 minutes, and the supernatant was transferred to a QuEChERS dispersive tube. The tubes were vortexed and centrifuged again for 5 minutes. The supernatant was transferred to a new $1.5 \mathrm{~mL}$ centrifuge tube to dry down under a nitrogen stream, at $50^{\circ} \mathrm{C}$, to dryness. The samples were reconstituted with $100 \mu \mathrm{L}$ of methanol and transferred to LC-MS/MS vials for analysis (Figure 3.3). 

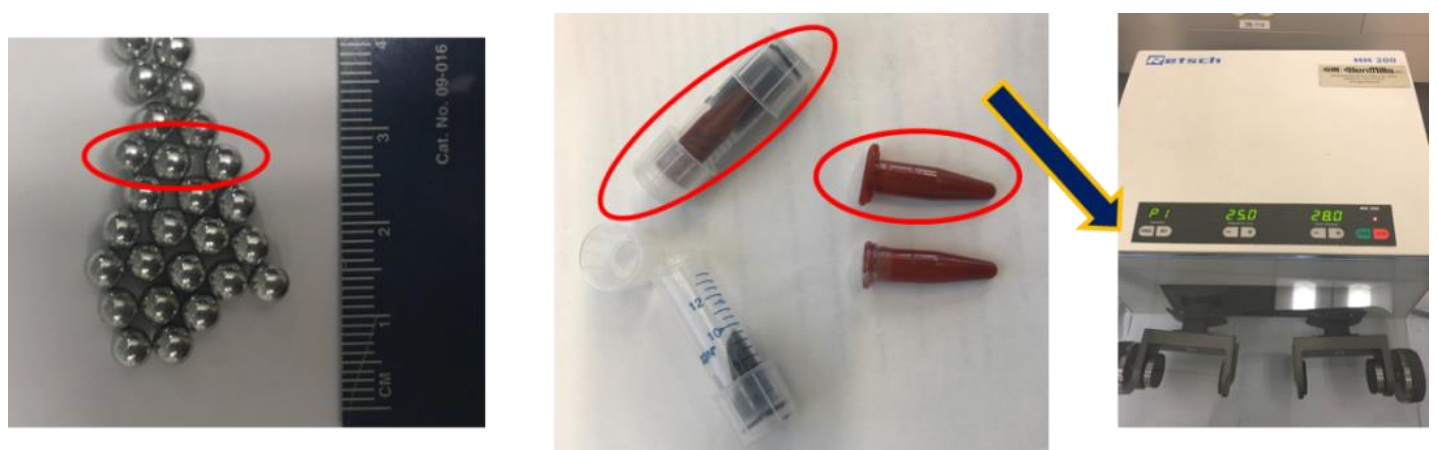

Figure 3.2: Images for the adapted homemade reaction chambers for the QuEChERS extraction.
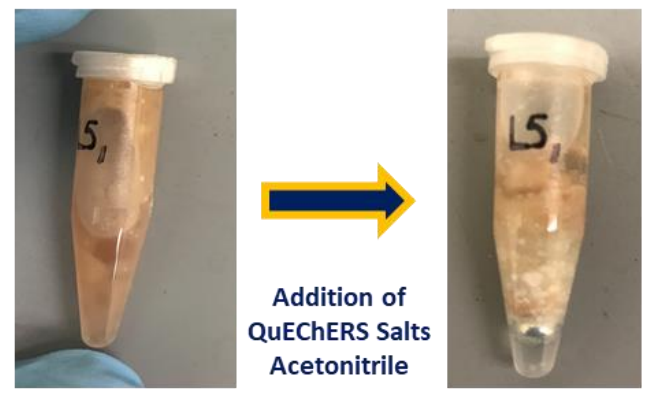

Homogenization

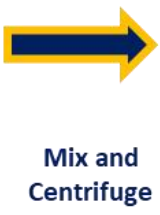

Centrifuge

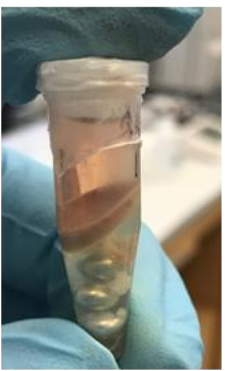

ransfer to Dispersive-SPE tube
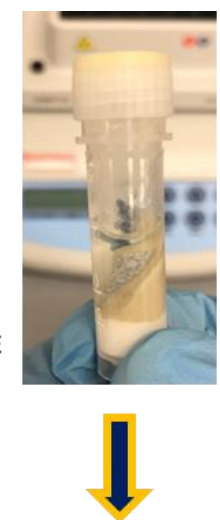

Pre-concentrate for LC-MS/MS Analysis

Figure 3.3: Visual workflow for the QuEChERS extraction.

\subsection{SPE}

Samples of $0.2 \mathrm{~g}$ tissue were placed into $1.5 \mathrm{~mL}$ centrifuge tubes, and then $200 \mu \mathrm{L}$ deionized water, $100 \mu \mathrm{L}$ internal standard (IS), and $400 \mu \mathrm{L} \mathrm{NaOH}$ were added to the tubes. The tubes were vortexed and placed in a $65^{\circ} \mathrm{C}$ water bath for 1 hour to incubate $(75,123)$. After incubation, the samples were centrifuged for 5 minutes and transferred to a $12 \mathrm{~mL}$ polypropylene tubes, and $200 \mu \mathrm{L}$ of $\mathrm{HCl}$ was added. Another $2 \mathrm{~mL}$ of deionized water was added to the samples which were then vortexed, and this completed the preparation of the sample for solid phase extraction. This hydrolysis pretreatment for SPE analysis was adapted from Huang et al. to assist the release of basic drugs from the particulate cell material (75). 
To each Bond Elut Certify column, $1 \mathrm{~mL}$ of deionized water was added and eluted under positive pressure. Then, $1 \mathrm{~mL}$ of methanol was added and eluted in the same manner in order to condition the columns. For loading, all of the $3 \mathrm{~mL}$ sample was loaded into each of the columns, then eluted under positive pressure.

To clean, $1 \mathrm{~mL}$ of deionized water was added and eluted, then $1 \mathrm{~mL}$ of $0.01 \mathrm{M} \mathrm{HCl}$ was added and eluted, and finally $2 \mathrm{~mL}$ of methanol was added and eluted, all in a similar manner.

For elution, $1 \mathrm{~mL}$ of $\mathrm{MeOH} / \mathrm{NH} 4 \mathrm{OH}(98: 2)$ solution was eluted. The loading, wash, and elution procedure was adapted from the publications of Jenkins et al. and Koesukwiwat et al. $(76,78)$. After the extraction, the eluate was evaporated under a nitrogen stream, at $50^{\circ} \mathrm{C}$, to dryness. This was then reconstituted with $100 \mu \mathrm{L}$ of methanol, and the samples were transferred to a LC-MS/MS vial for analysis.

\subsection{LLE}

Samples of $0.2 \mathrm{~g}$ tissue were placed in a $15 \mathrm{~mL}$ conical centrifuge polypropylene tube, and then $200 \mu \mathrm{L}$ deionized water, $100 \mu \mathrm{L} \mathrm{IS}, 50 \mu \mathrm{L} \mathrm{NaOH}$, and $1.5 \mathrm{~mL}$ methyl tertbutyl ether (MTBE) were added to the tubes. MTBE was chosen as the organic solvent based on the successful extraction of fentanyl from blood and plasma in previous published $\operatorname{LLE}$ work $(124,125)$. The tubes were vortexed, and then centrifuged for 5 minutes. After centrifugation, the supernatant was transferred to a $1.5 \mathrm{~mL}$ microcentrifuge tube and the samples were evaporated under a nitrogen stream, at $50^{\circ} \mathrm{C}$, to dryness. The sample was reconstituted with $100 \mu \mathrm{L}$ of methanol and transferred to a LC-MS/MS vial for analysis (Figure 3.4). 


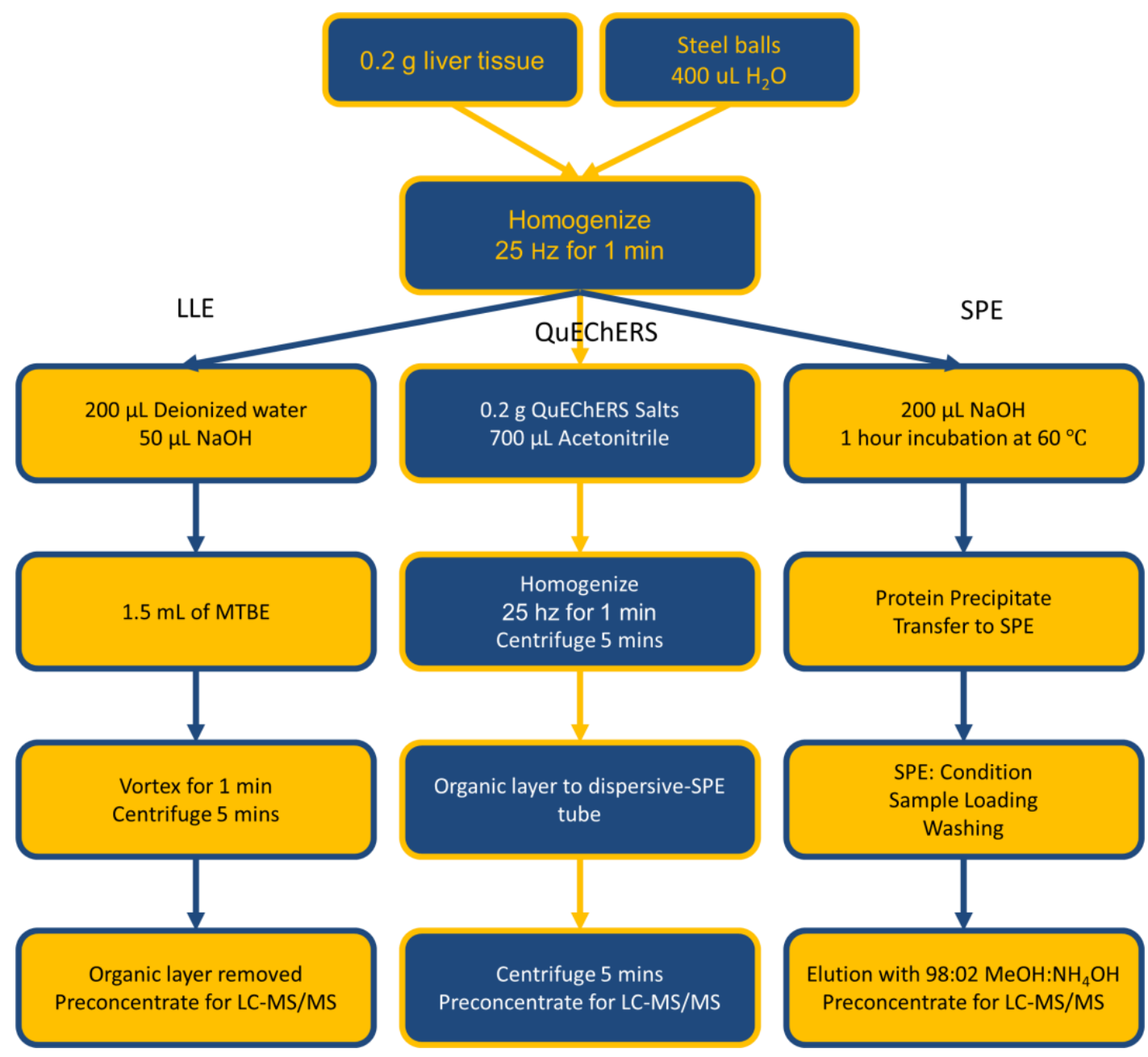

Figure 3.4: Workflows for the LLE, SPE, and QuEChERS extraction protocols.

\subsubsection{Extraction Validation}

\subsection{Selectivity}

The three analytes of interest and two deuterated internal standards were analyzed individually at a concentration of $1 \mu \mathrm{g} / \mathrm{mL}$ using the MRM method to examine any interfering peaks. Aliquots of the pooled beef liver specimens and 10 human liver specimens were extracted and analyzed to ensure no interferences were present for the target analytes and internal standards. A negative control, blank matrix with internal standard mix spike, was analyzed each run to determine any potential interferences. Chromatographic analysis of these compounds was performed using Agilent MassHunter Qualitative Analysis software version B.08.00. 
Potential for interference from other drug classes was evaluated by analysis of liver spiked with a mix of 31 compounds incorporating other opioids, stimulants, depressants, synthetic cannabinoids, synthetic cathinones, and supplements shown in Table 3.2 .

Table 3.2: List of 31 compounds evaluated for potential interferences

\begin{tabular}{|c|c|c|c|}
\hline Class & Compound & Class & Compound \\
\hline \multirow[t]{6}{*}{ Opioids } & 6-Acetylmorphine & Synthetic & JWH-018 \\
\hline & Oxycodone & Cannabinoids & JWH-073 \\
\hline & Hydrocodone & & XLR-11 \\
\hline & Buprenorphine & & AB-FUBINACA \\
\hline & Norbuprenorphine & & AB-PINACA \\
\hline & Ethylmorphine & & MAM2201 \\
\hline \multirow[t]{2}{*}{ Opiates } & Morphine & Stimulants & Amphetamine \\
\hline & Codeine & & Methamphetamine \\
\hline \multirow[t]{7}{*}{ Supplements } & 1S,2R (+)-Ephedrine & & Cocaine \\
\hline & Methylphenidate & Synthetic Cathinones & $\begin{array}{c}\text { Alpha- } \\
\text { Pyrrolidinopentiophenone } \\
\text { (A-PVP) }\end{array}$ \\
\hline & Sibutramine & & $\begin{array}{c}\text { 3,4-Methylenedioxy } \\
\text { Pyrovalerone } \\
\text { (MDPV) }\end{array}$ \\
\hline & Caffeine & Cannabinoids & Delta-9 THC (THC) \\
\hline & Synephrine & & Cannabidiol (CBD) \\
\hline & Octopamine & Other Alkaloids & Mitragynine \\
\hline & $\begin{array}{l}\text { Methylhexanamine } \\
\text { (DMAA) }\end{array}$ & & \\
\hline
\end{tabular}

\subsection{Ionization Suppression/Enhancement}

Ionization suppression or enhancement was evaluated at two concentrations, low and high, for 10 replicates at each concentration. Using a post-extraction addition approach, analyte peak areas of neat standards (Set 1) are compared to matrix samples fortified with neat standards after extraction (Set 2). Set 1 consisted of neat standards prepared at 1 (low) and 10 (high) $\mu \mathrm{g} / \mathrm{kg}$ and injected 6 times to establish a mean peak area for each concentration. Set 2 consisted of ten $0.2 \mathrm{~g}$ aliquots of beef liver, for each low and high concentration, extracted and spiked with the low and high concentration respectively. The 
average area of each set $(\bar{X})$ was used to calculate the suppression or enhancement effect at each concentration for each analyte is as follows:

$$
\text { Ionization suppression or enhancement }(\%)=\left(\frac{\bar{X} \text { Area of set } 2}{\bar{X} \text { Area of set } 1}-1\right) * 100
$$

\subsection{Bias and Precision}

QC samples were analyzed at LOW, MED, and HIGH for fentanyl, norfentanyl, and 4-ANPP in triplicate over five days. The source of liver in this parameter evaluation was pooled beef liver $(n=6)$ which is different from the standard 036. The ASB 036 encourages the use of matrix matched comparisons however beef liver was used as the model for the bias and precision studies. To account for these differences, further studies in the proof of applicability were performed to compare the impact of beef liver to human liver. Bias was calculated as a percent deviation of the mean from the theoretical concentration. Precision was evaluated by percent relative standard deviation $(\% \mathrm{CV})$ of the mean at each concentration. The criteria for acceptable bias and precision (\%CV) was set at $\pm 20 \%$.

\subsection{Linearity of Calibration}

Aliquots of $0.2 \mathrm{~g}$ of pooled beef liver were spiked with $5 \mu \mathrm{L}$ of corresponding solutions to create calibration samples at $0.5,1,2.5,10,50$, and $100 \mu \mathrm{g} / \mathrm{kg}$ for fentanyl and norfentanyl with corresponding concentrations of $0.4,0.8,2,8,40$, and $80 \mu \mathrm{g} / \mathrm{kg}$ for 4-ANPP. Five replicates at each concentration were analyzed and regression lines were generated using Agilent MassHunter software using a weight of (1/x).

\subsection{Processed Sample Stability}

To determine the stability of processed samples in the autosampler $\left(4^{\circ} \mathrm{C}\right)$, aliquots of beef liver were spiked at low $(1 \mu \mathrm{g} / \mathrm{kg})$ and high $(10 \mu \mathrm{g} / \mathrm{kg})$ concentrations followed by extraction. Triplicate analysis at time frames: $0,24,48$, and 72 hours were performed for each concentration. Average peak area was calculated for each triplicate and compared to time zero. An acceptable bias threshold was set at $\pm 20 \%$. 


\subsection{Limits}

To evaluate the limit of detection (LOD) and lower limit of quantitation (LLOQ), aliquots of blank liver were spike with decreasing concentrations of analytes. To determine LLOQ, triplicates at $0.4 \mu \mathrm{g} / \mathrm{kg}$ 4-ANPP and $0.5 \mu \mathrm{g} / \mathrm{kg}$ concentration for fentanyl and norfentanyl were analyzed over 3 days ( $\mathrm{n}=9$ ). Bias and precision (\%CV) for LLOQ was considered acceptable at $\pm 20 \%$. To determine LOD, triplicates at 0.08 $\mu \mathrm{g} / \mathrm{kg}$ 4-ANPP and $0.1 \mu \mathrm{g} / \mathrm{kg}$ concentration for fentanyl and norfentanyl were analyzed over 2 days ( $\mathrm{n}=6$ ). For LOD to be considered acceptable, signal to noise $(s / n)$ ratios were to be above 3.3 and qualifier ratios within $\pm 20 \%$ to achieve chromatographic acceptance.

\subsection{Proof of Applicability}

Liver specimens from twelve authentic postmortem cases provided by the OCME

were submitted for analysis. Samples were analyzed using the QuEChERS extraction protocol presented above. A comparison of spiked beef liver and spiked human liver at low, medium, and high concentrations was also performed to display the methods ability to quantify the target analytes in human liver matrix.

\subsubsection{Comparison of Extraction Techniques (Recovery, Matrix Effects, and Process Efficiency)}

To calculate matrix effects (ME), recovery of the extraction (RE), and process efficiency (PE) three

formulas were utilized. Variables in the formulas represent the mean peak areas for the neat solution (A), the mean peak areas for the standards spiked after extraction (B), and the mean peak areas for the standards spiked before extraction (C)(39). Recovery, matrix effects, and process efficiency were used to compare extraction methodologies for LLE, SPE, and the validated QuEChERS method. Low and high concentrations were analyzed by all three techniques at five replicates $(n=5)$.

$$
\begin{aligned}
& M E(\%)=\frac{B}{A} * 100 \\
& R E(\%)=\frac{C}{B} * 100 \\
& P E(\%)=\frac{C}{A} * 100
\end{aligned}
$$




\subsubsection{Results and Discussion}

\subsubsection{Selectivity}

With the MRM method, no interfering peaks were observed when the target analytes or internal standards were analyzed individually. After analysis of the pooled liver specimens and 10 human liver specimens, no interfering peaks were identified for the target analytes or internal standards. Figure 3.5 below shows a chromatogram for the three target analytes after extraction of the lowest calibrator ( 0.5 $\mu \mathrm{g} / \mathrm{kg}$ ) superimposed with the extraction of a blank matrix showing no interference peaks. Analysis of liver spiked with a mix of 31 potential interfering drugs (Table 3.2) presented no evidence of interference. Based on the full analysis of the single analysis, matrix extractions, and interference drug mix the MRM method was deemed highly selective and no interference peaks were identified.

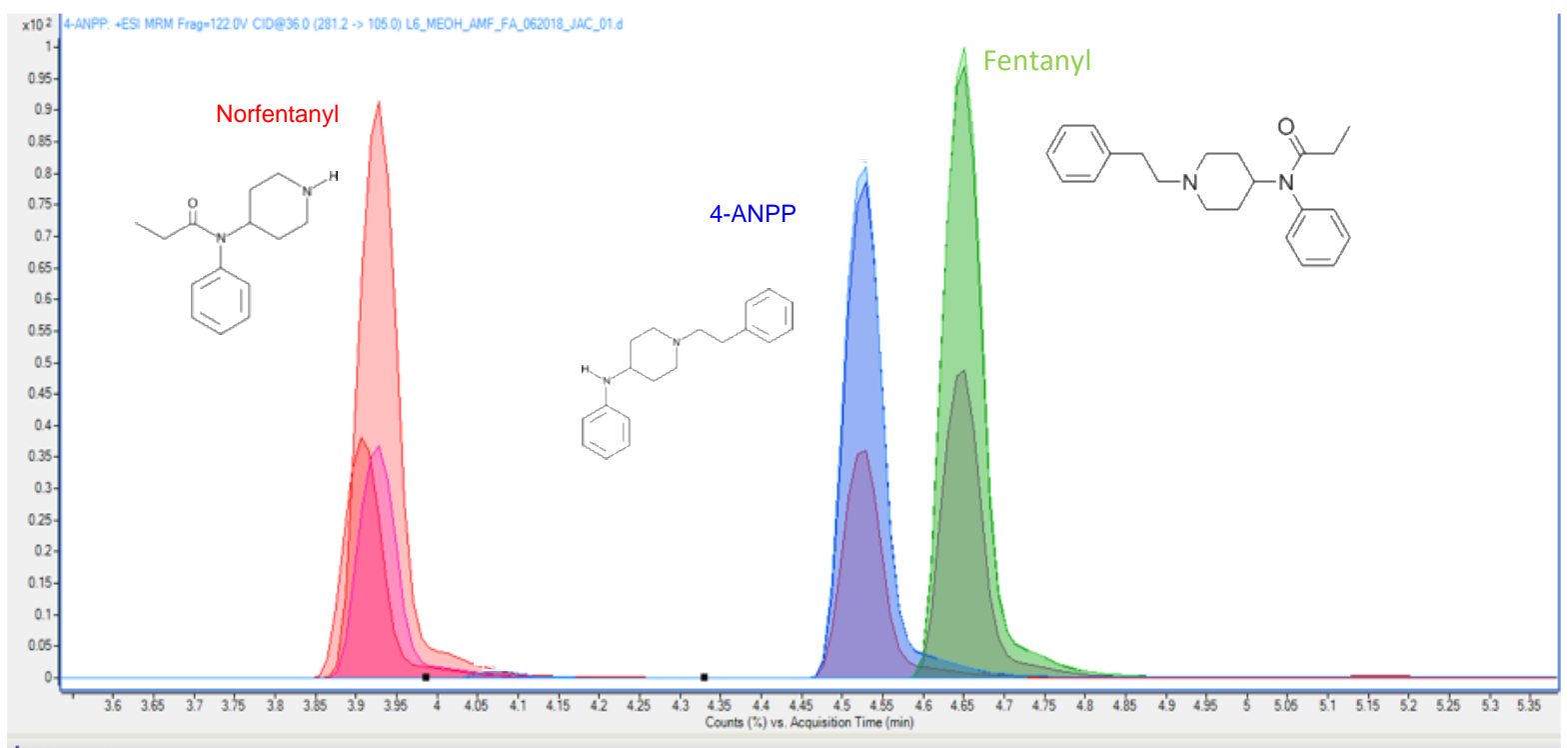

Figure 3.5: LC-MS/MS chromatogram for the MRM transitions of the three target analytes.

\subsubsection{Ionization Suppression/Enhancement}

Ionization suppression or enhancement was evaluated at two concentrations, low and high, for 10 replicates at each concentration following the ASB Standard 036 proposed requirements. The percent ionization of suppression or enhancement for each target analyte as well as \%CV are displayed in Table 3.3. Suppression or enhancement did not exceed $\pm 10 \%$, well within the ASB Standard 036 proposed criteria of $\pm 25 \%$ with $\% \mathrm{CV}$ not exceeding $\pm 20 \%$. For interpretation of the ionization results, a negative 
percent ionization is indicative of suppression and a positive percent is indicative of enhancement. A value of $0 \%$ is interpreted as no enhancement or suppression. Norfentanyl experienced enhancement at both low and high concentrations while inversely 4-ANPP experienced suppression at low and high concentrations. At low concentration, fentanyl experienced suppression and at the high a slight enhancement. Based on the evaluation, the extraction method and subsequent LC-MS/MS analysis experiences acceptable ionization suppression and enhancement.

Table 3.3: Ionization Suppression/Enhancement.

\begin{tabular}{|c|c|c|c|c|}
\hline \multirow{3}{*}{ Compound } & \multicolumn{4}{|c|}{ Ionization Suppression/Enhancement } \\
\hline & \multicolumn{2}{|c|}{ Low $1 \mu \mathrm{g} / \mathrm{kg}$} & \multicolumn{2}{|c|}{ High $10 \mu \mathrm{g} / \mathrm{kg}$} \\
\hline & Mean \% & $\% \mathrm{CV}$ & Mean $\%$ & $\% \mathrm{CV}$ \\
\hline Fentanyl & -10.0 & 8.0 & 1.4 & 17.5 \\
\hline 4-ANPP & -2.1 & 16.0 & -0.3 & 11.6 \\
\hline Norfentanyl & 3.1 & 16.1 & 2.8 & 9.5 \\
\hline
\end{tabular}

\subsubsection{Bias and Precision}

QC samples were analyzed at LOW $(1.0 \mu \mathrm{g} / \mathrm{kg})$, MED $(10 \mu \mathrm{g} / \mathrm{kg})$, and HIGH $(50 \mu \mathrm{g} / \mathrm{kg})$ for fentanyl and norfentanyl with corresponding concentrations of LOW $(0.8 \mu \mathrm{g} / \mathrm{kg}), \operatorname{MED}(8 \mu \mathrm{g} / \mathrm{kg})$, and HIGH $(40$ $\mu \mathrm{g} / \mathrm{kg}$ ) for 4-ANPP in triplicate over five days $(\mathrm{n}=15)$. Table 3.4 displays the bias and precision results from the analysis of these concentrations. The bias for all three target analytes at all three concentrations did not exceed $\pm 20 \%$. Displayed below is repeatability (intra-day $\% \mathrm{CV}$ ), reproducibility (inter-day $\% \mathrm{CV}$ ), and bias. The analyte with the least bias was norfentanyl at $1.5 \%$ and was the analyte with the least bias ( $< \pm 8.5 \%)$. Bias for 4 -ANPP was between $-19.9 \%$ and $-14.7 \%$ which is within the acceptable $\pm 20 \%$ however much higher than the other analytes at all three concentrations. A possible reason for this is the lack of use of a deuterated standard as the IS for 4-ANPP. Future work will include the use of D5-4-ANPP to attempt to alleviate this issue. 
Table 3.4: Bias and Precision

\begin{tabular}{|c|c|c|c|c|c|c|c|c|c|}
\hline \multirow{2}{*}{ Compound } & \multicolumn{3}{|c|}{ Repeatability (\%CV) } & \multicolumn{3}{|c|}{ Reproducibility (\%CV) } & \multicolumn{3}{|c|}{ Bias $(\%)$} \\
\hline & LOW & MED & HIGH & LOW & MED & $\mathrm{HIGH}$ & LOW & MED & $\mathrm{HIGH}$ \\
\hline Fentanyl & 3.7 & 3.6 & 9.5 & 2.2 & 3.6 & 15.1 & -15.8 & -3.6 & -2.5 \\
\hline 4-ANPP & 13.8 & 6.9 & 8.3 & 3.7 & 9.5 & 4.9 & -19.9 & -14.7 & -16.1 \\
\hline Norfentanyl & 3.7 & 4.6 & 9.9 & 12.2 & 6.6 & 11.9 & -8.5 & -5.3 & 1.5 \\
\hline
\end{tabular}

\subsubsection{Linearity of Calibration}

For the calibration curves 6 levels were employed: $0.4,0.8,2,8,40$, and $80 \mu \mathrm{g} / \mathrm{kg}$ for 4 -ANPP and $0.5,1,2.5,10,50$, and $100 \mu \mathrm{g} / \mathrm{kg}$ for fentanyl and norfentanyl. Five replicates at each concentration were analyzed and regression lines were generated using Agilent MassHunter software using a weight of $(1 / x)$. Table 3.5 below displays results for the five replicates. The $\mathrm{R}^{2}$ values for the calibration curve of the three target analytes all exceeded 0.997 for the analytical measurable range (AMR) of $0.4-80 \mu \mathrm{g} / \mathrm{kg}$ for $4-\mathrm{ANPP}$ and $0.5-100 \mu \mathrm{g} / \mathrm{kg}$ for fentanyl and norfentanyl.

Table 3.5: Linearity of calibration.

\begin{tabular}{lcccccc}
\hline Compound & $\begin{array}{c}\mathrm{y} \text {-intercept } \\
(\mathrm{m} e a n \pm \mathrm{SD}, \mathrm{n}=5)\end{array}$ & $\begin{array}{c}\text { Slope } \\
(\mathrm{mean} \pm \mathrm{SD}, \mathrm{n}=5)\end{array}$ & $\begin{array}{c}\mathrm{R}^{2} \text { range } \\
(\mathrm{n}=5)\end{array}$ & $\begin{array}{c}\mathrm{LOD} \\
(\mathrm{ug} / \mathrm{kg})\end{array}$ & $\begin{array}{c}\mathrm{LLOQ} \\
(\mathrm{ug} / \mathrm{kg})\end{array}$ & $\begin{array}{c}\text { AMR } \\
(\mathrm{ug} / \mathrm{kg})\end{array}$ \\
\hline Fentanyl & $0.0142 \pm 0.0026$ & $0.0222 \pm 0.0007$ & $\begin{array}{c}0.9974- \\
0.9984\end{array}$ & 0.1 & 0.5 & $0.5-100$ \\
4-ANPP & $0.0102 \pm 0.0058$ & $0.0199 \pm 0.0010$ & $\begin{array}{c}0.9973- \\
0.9999\end{array}$ & 0.1 & 0.4 & $0.4-80$ \\
Norfentanyl & $0.0125 \pm 0.0026$ & $0.0205 \pm 0.0006$ & $\begin{array}{c}0.9978- \\
0.9982\end{array}$ & 0.1 & 0.5 & $0.5-100$ \\
\hline
\end{tabular}

\subsubsection{Processed Sample Stability}

To determine the stability of processed samples, twelve $0.2 \mathrm{~g}$ aliquots of beef liver were spiked at low $(1 \mu \mathrm{g} / \mathrm{kg})$ and high $(10 \mu \mathrm{g} / \mathrm{kg})$ concentrations followed by extraction. The twelve reconstituted extracts for each concentration were combined and again aliquoted to LC-MS/MS vials for analysis. This allowed for triplicate analysis at four-time frames: $0,24,48$, and 72 hours for each concentration. Average peak area was calculated for each triplicate and compared to time zero. The percent change from the initial relative response to the time point analyzed for each target analyte is displayed in Table 3.6 below. 
The percent change threshold was established as the acceptable bias, therefore a $\pm 20 \%$ change was deemed acceptable for this method. A graph of the stability for the three target analytes is shown in Figure 3.6. For the time points analyzed, none exceeded the $\pm 20 \%$ change criteria with the highest of $16.8 \%$ and the lowest $-1.6 \%$. The three target analytes are stable in the autosampler $\left(4^{\circ} \mathrm{C}\right)$ for up to 72 hours.

Table 3.6: Processed stability for samples stored in autosampler $\left(4^{\circ} \mathrm{C}\right)$.

\begin{tabular}{|c|c|c|c|c|c|c|}
\hline \multirow[t]{2}{*}{ Compound } & \multicolumn{3}{|c|}{ Low $(\% \Delta)^{\mathrm{a}}$} & \multicolumn{3}{|c|}{$\operatorname{High}(\% \Delta)^{\mathrm{a}}$} \\
\hline & $24 \mathrm{~h}^{\mathrm{b}}$ & $48 h^{b}$ & $72 \mathrm{~h}^{\mathrm{b}}$ & $24 \mathrm{~h}^{\mathrm{b}}$ & $48 \mathrm{~h}^{\mathrm{b}}$ & $72 \mathrm{~h}^{\mathrm{b}}$ \\
\hline Fentanyl & 1.5 & 1.8 & 4.3 & 1.9 & -1.1 & 0.6 \\
\hline 4-ANPP & 5.1 & 9.8 & 7.7 & 0.6 & 4.9 & 4.1 \\
\hline Norfentanyl & 0.6 & 8.2 & 16.8 & -1.6 & -0.7 & -1.6 \\
\hline
\end{tabular}

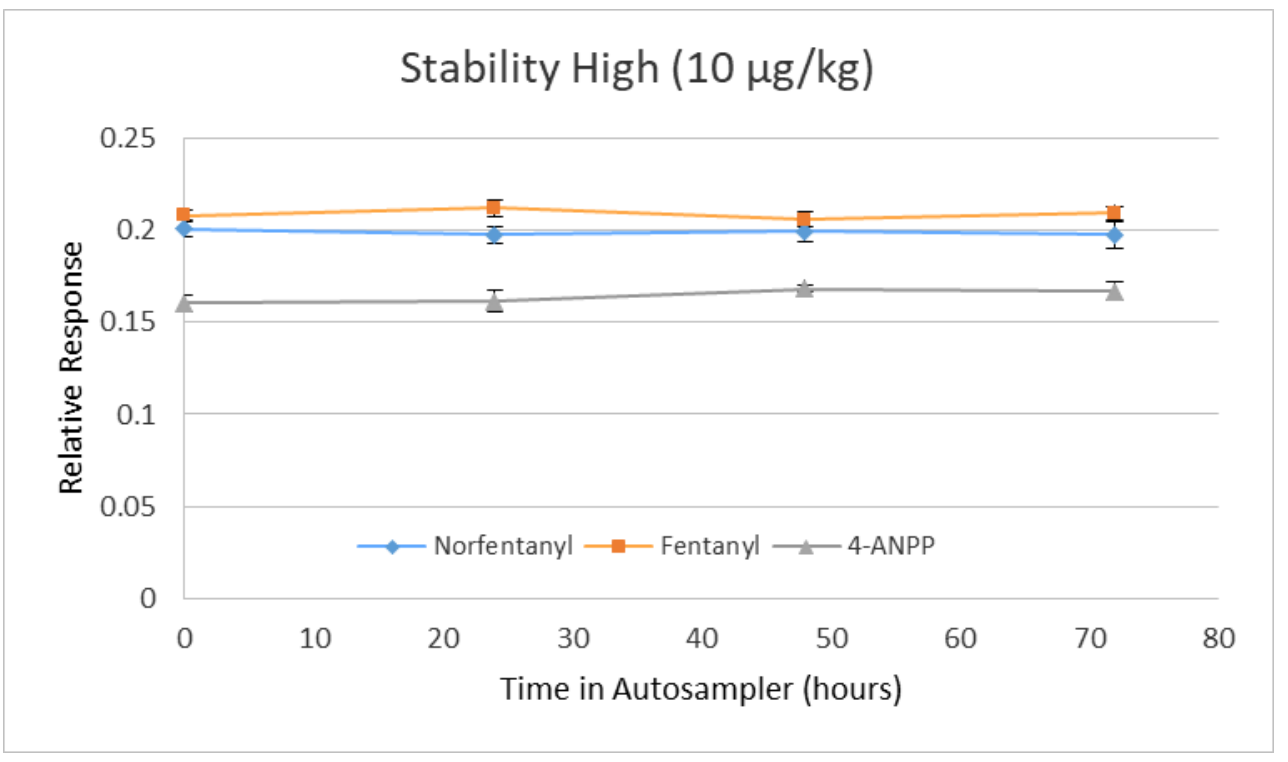

Figure 3.6: Graphical representation of the stability of the high concentration for processed samples stored in the autosampler $\left(4^{\circ} \mathrm{C}\right)$.

\subsubsection{Limits}

To determine LLOQ, triplicates at $0.4 \mu \mathrm{g} / \mathrm{kg} 4-\mathrm{ANPP}$ and $0.5 \mu \mathrm{g} / \mathrm{kg}$ concentration for fentanyl and norfentanyl were analyzed over 3 days $(n=9)$. Bias for all three target compounds did not exceed the $\pm 20 \%$ acceptable criteria and the \%CV was within the acceptable criteria. Bias for fentanyl, norfentanyl, and 4ANPP were $-10.3,1.3$, and -14.5 respectively with $\%$ CV values of $4.9 \%, 5.8 \%$, and $4.0 \%$ respectively. To 
determine LOD, triplicates at $0.08 \mu \mathrm{g} / \mathrm{kg}$ 4-ANPP and $0.1 \mu \mathrm{g} / \mathrm{kg}$ concentration for fentanyl and norfentanyl were analyzed over 2 days ( $n=6)$. The LOD calculated to be $0.1 \mu \mathrm{g} / \mathrm{kg}$ for all three analytes by achieving the acceptable signal to noise $(s / n)$ ratios above 3.3 and qualifier ratios within $\pm 20 \%$ for chromatographic acceptance.

\subsubsection{Proof of Applicability}

Of the liver specimens from the twelve authentic postmortem cases provided by the OCME, 10 were positive for fentanyl and norfentanyl while 7 were also positive for 4-ANPP. Figure 3.7 displays the quantitation results for the OCME liver samples. The range of fentanyl concentrations were 56.6 to 462.3 $\mu \mathrm{g} / \mathrm{kg}$ with a mean of $149.2 \mu \mathrm{g} / \mathrm{kg}(\mathrm{n}=10)$. The range of norfentanyl concentrations were 1.9 to $50.0 \mu \mathrm{g} / \mathrm{kg}$ with a mean of $14.1 \mu \mathrm{g} / \mathrm{kg}(\mathrm{n}=10)$. The range of 4-ANPP concentrations were 3.2 to $23.7 \mu \mathrm{g} / \mathrm{kg}$ with a mean of $7.5 \mu \mathrm{g} / \mathrm{kg}(\mathrm{n}=7)$.

The concentrations reported in this study are in an agreement with the concentrations reported by McIntyre, Luckenbill, and Palamalai $(64,65,115)$. A review by McIntyre found that the postmortem central blood concentrations of fentanyl detected in 179 cases had a range of $1.8-176 \mathrm{ng} / \mathrm{mL}$ with an average of $20.61 \mathrm{ng} / \mathrm{mL}$ (115). In the same review, 87 liver fentanyl concentrations from cases in Los Angeles had a range of $6.9-689 \mu \mathrm{g} / \mathrm{kg}$ with a mean of $93.3 \mu \mathrm{g} / \mathrm{kg}$ while 16 liver fentanyl concentrations from cases in San Diego had a range of 3.8-270 $\mu \mathrm{g} / \mathrm{kg}$ with an average of $74.1 \mu \mathrm{g} / \mathrm{kg}$ (115). A similar study by Luckenbill found fentanyl concentrations in 9 liver tissue samples in a range from 37-179 $\mu \mathrm{g} / \mathrm{kg}$ with a mean of $83.1 \mu \mathrm{g} / \mathrm{kg}$ and in 3 heart tissue samples ranging from $52.8-179 \mu \mathrm{g} / \mathrm{kg}$ with a mean of $124 \mu \mathrm{g} / \mathrm{kg}$ (65). Palamalai presented a review of cases that fentanyl was detected at postmortem investigations that deaths were deemed non-drug related, mix drug related or fentanyl caused deaths. Those findings consisted of liver fentanyl concentrations in five non-drug related deaths with a range of $11-104 \mu \mathrm{g} / \mathrm{kg}$ and a mean of $38 \mu \mathrm{g} / \mathrm{kg}$; liver fentanyl concentrations in 26 mix drug related deaths ranging from 6-235 $\mu \mathrm{g} / \mathrm{kg}$ and a mean of $80 \mu \mathrm{g} / \mathrm{kg}$; and liver fentanyl concentrations in 33 fentanyl related deaths ranging from $18-365 \mu \mathrm{g} / \mathrm{kg}$ and a mean of $104 \mu \mathrm{g} / \mathrm{kg}(64)$. The values for the studies are displayed in Table 3.7. 
The method presented by the authors covers an analytical range fit for the purpose of analysis of fentanyl and the two major metabolites.

Table 3.7: Review of fentanyl liver concentrations $(\mu \mathrm{g} / \mathrm{kg})$.

\begin{tabular}{lcccc}
\hline Author & $\mathrm{N}$ & Low & High & Mean \\
\hline Luckenbill-2008 & 9 & 37 & 179 & 124 \\
$\begin{array}{l}\text { McIntyre-2012 } \\
\text { (Los Angeles) }\end{array}$ & 87 & 6.9 & 689 & 93.3 \\
$\begin{array}{l}\text { McIntyre-2012 } \\
\text { (San Diego) }\end{array}$ & 16 & 3.8 & 270 & 74.1 \\
$\begin{array}{l}\text { Palamalai-2013 } \\
\text { (Non-drug related) }\end{array}$ & 5 & 11 & 104 & 38 \\
$\begin{array}{l}\text { Palamalai-2013 } \\
\text { (Mix drug related) }\end{array}$ & 26 & 6 & 235 & 80 \\
$\begin{array}{l}\text { Palamalai-2013 } \\
\text { (Fentanyl COD }{ }^{1} \text { ) }\end{array}$ & 33 & 18 & 365 & 104 \\
$\begin{array}{l}\text { This Study } \\
\text { Cause of death }\end{array}$ & 10 & 56.6 & 462 & 149 \\
\hline
\end{tabular}

For the comparison of beef liver and human liver as a matrix, the bias for both matrices at all three concentrations (LOW, MED, and HIGH) was below $\pm 13 \%$ with a $\%$ CV below $13.8 \%(n=5)$. Table 3.8 displays the results for the bias, precision, and statistical test to compare the bias for the beef and human liver extractions. A paired T-test (at a 95\% confidence interval) was performed to compare the bias for the beef and human liver extractions with all p-values generated above 0.05 indicating no significant differences between the two groups. Based on this data the is not a significant difference between the beef liver and human liver extractions allowing for application to human liver extractions. 


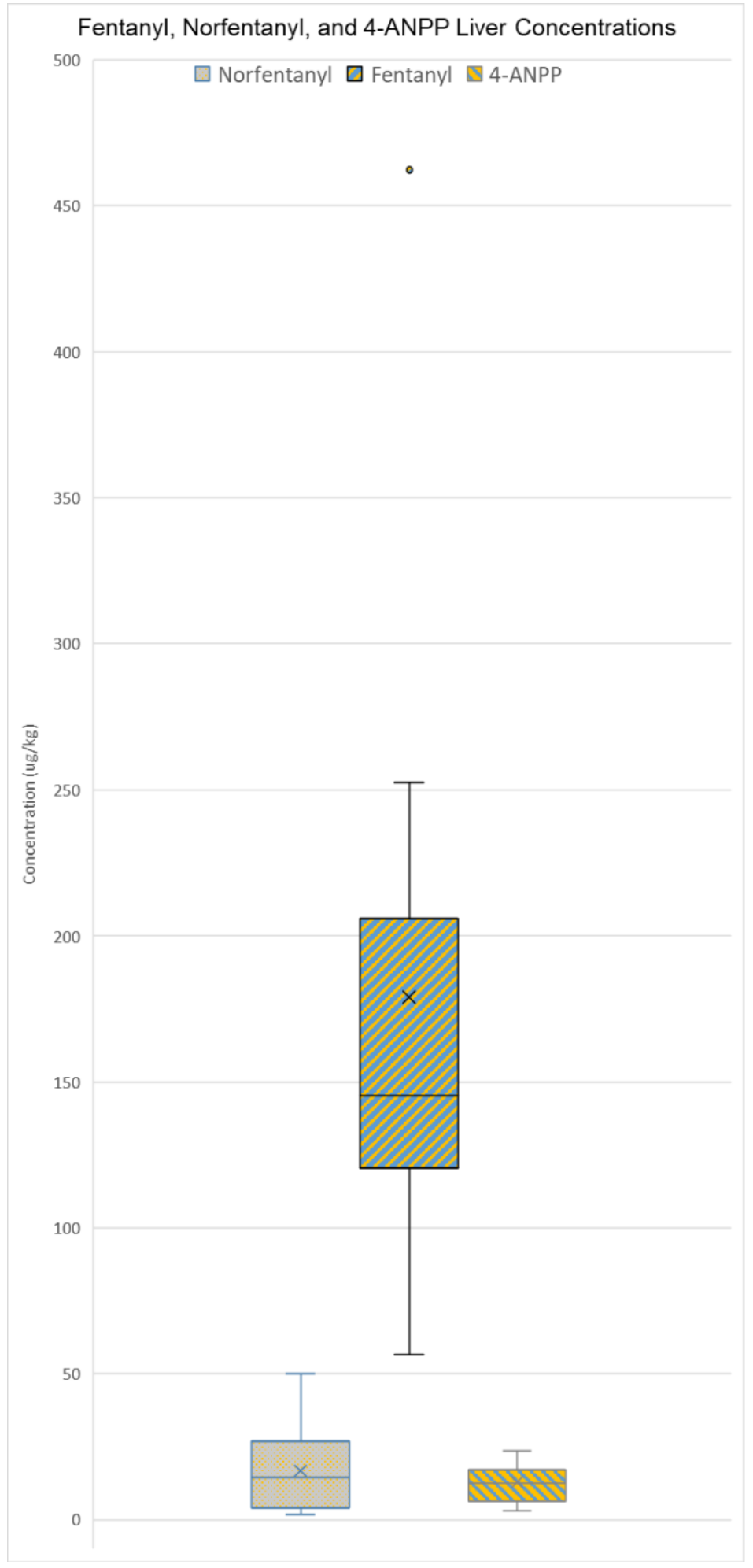

Figure 3.7: Box plot of quantitative results for the OCME case samples. 
Table 3.8: Comparison of beef and human liver extractions at low, med, and high concentrations $(n=5)$.

\begin{tabular}{|c|c|c|c|c|c|c|c|c|c|}
\hline \multirow{3}{*}{ Compound } & \multicolumn{6}{|c|}{ Bias and Precision } & \multicolumn{3}{|c|}{ T-test ${ }^{\mathrm{a}}$} \\
\hline & \multicolumn{2}{|c|}{ Low } & \multicolumn{2}{|c|}{ Med } & \multicolumn{2}{|c|}{ High } & \multirow{2}{*}{$\frac{\text { Low }}{\text { P-value }}$} & \multirow{2}{*}{$\frac{\text { Med }}{\text { P-value }}$} & \multirow{2}{*}{$\frac{\text { High }}{\text { P-value }^{c}}$} \\
\hline & Bias $^{\mathrm{b}}$ & $\mathrm{CV}^{\mathrm{b}}$ & Bias $^{b}$ & $\mathrm{CV}^{\mathrm{b}}$ & Bias $^{b}$ & $\mathrm{CV}^{\mathrm{b}}$ & & & \\
\hline \multicolumn{10}{|c|}{ Beef Liver } \\
\hline Fentanyl & -9.1 & 11.4 & 12.3 & 11.6 & 5.5 & 7.2 & 0.27 & 0.37 & 0.09 \\
\hline 4-ANPP & -4.4 & 13.8 & 13.0 & 10.6 & 2.8 & 5.3 & 0.34 & 0.51 & 0.90 \\
\hline Norfentanyl & -7.5 & 11.3 & 11.7 & 11.6 & 0.1 & 7.0 & 0.15 & 0.12 & 0.56 \\
\hline \multicolumn{10}{|c|}{ Human Liver } \\
\hline Fentanyl & 3.2 & 5.2 & 11.4 & 4.3 & 2.9 & 1.7 & & & \\
\hline 4-ANPP & 5.2 & 10.1 & 8.5 & 6.0 & -2.4 & 0.9 & & & \\
\hline Norfentanyl & 0.2 & 7.1 & 6.3 & 6.7 & -5.2 & 1.7 & & & \\
\hline
\end{tabular}

\subsubsection{Comparison of Extraction Techniques}

Recovery, matrix effects, and process efficiency were used to compare extraction methodologies

for LLE, SPE, and the validated QUEChERS method. Low and high concentrations were analyzed by all three techniques at five replicates $(n=5)$ and the results are displayed in Table 3.9. The lowest recovery for 4ANPP using the QuEChERS, LLE, and SPE techniques were $90.2 \%, 66.3 \%$, and $46.4 \%$ respectively. Overall, the QuEChERS technique met all the acceptable criteria set by Matuszewski et al. with recoveries higher than $90.2 \%$, matrix effects greater than $80.4 \%$, and process efficiencies greater than $76.7 \%$ with $\% \mathrm{CV}$ values below $12.8 \%$ for all target analytes (107). The LLE and SPE techniques failed to meet acceptable criteria for recoveries $( \pm 20 \%)$ and matrix effects $( \pm 25 \%)$ at multiple concentrations for multiple analytes. The mean recovery for the QuEChERS extraction was $94.5 \%$ with a range of $90.2 \%$ to $97.2 \%$; the mean recovery for the LLE method was $88.2 \%$ with a range of $78.2 \%$ to $99.8 \%$, and the mean recovery for the SPE method was $86.3 \%$ with a range of $57.9 \%$ to $112.5 \%$. The QuEChERS technique generated the highest mean recovery with the smallest range of recoveries compared to the LLE and SPE techniques presented in this study. The process efficiency for the QuEChERS extraction was above $76 \%$ for all three analytes compared to the LLE and SPE methods that only produced a process efficiency above $76 \%$ for norfentanyl. 
Process efficiency compares the spiked pre-extract specimen with the neat specimen, incorporating the entire extraction process including both recovery and matrix effect

into the percent. A desired PE is $\pm 25 \%$ and the QuEChERS extraction is the only method of the three presented in this study that achieved it for all three analytes.

Table 3.9: Matrix effects, recovery, and process efficiency for the 3 extraction techniques $(n=5)$.

\begin{tabular}{|c|c|c|c|c|c|c|c|c|c|c|c|c|}
\hline \multirow{3}{*}{ Compound } & \multicolumn{4}{|c|}{ Matrix Effects ${ }^{a}$} & \multicolumn{4}{|c|}{ Recovery $^{\mathrm{a}}$} & \multicolumn{4}{|c|}{ Process Efficiency } \\
\hline & \multicolumn{2}{|c|}{ Low } & \multicolumn{2}{|c|}{ High } & \multicolumn{2}{|c|}{ Low } & \multicolumn{2}{|c|}{ High } & \multicolumn{2}{|c|}{ Low } & \multicolumn{2}{|c|}{ High } \\
\hline & Mean & $\mathrm{CV}$ & Mean & $\mathrm{CV}$ & Mean & $\mathrm{CV}$ & Mean & $\mathrm{CV}$ & Mean & $\mathrm{CV}$ & Mean & $\mathrm{CV}$ \\
\hline \multicolumn{13}{|c|}{ QuEChERS } \\
\hline Fentanyl & 80.4 & 2.9 & 89.3 & 1.8 & 97.2 & 12.4 & 95.1 & 7.3 & 78.1 & 12.1 & 98.7 & 7.2 \\
\hline 4-ANPP & 85.0 & 5.2 & 95.2 & 2.4 & 90.2 & 9.0 & 95.1 & 12.8 & 76.7 & 8.7 & 95.2 & 2.4 \\
\hline Norfentanyl & 87.0 & 1.9 & 111.0 & 3.6 & 96.0 & 5.9 & 117.0 & 6.2 & 83.5 & 5.9 & 117.0 & 6.3 \\
\hline \multicolumn{13}{|c|}{ LLE } \\
\hline Fentanyl & 65.3 & 17.3 & 72.0 & 8.8 & 78.2 & 10.8 & 75.9 & 8.8 & 51.1 & 19.1 & 73.4 & 8.3 \\
\hline 4-ANPP & 62.1 & 16.9 & 66.4 & 11.2 & 86.5 & 11.9 & 66.3 & 10.4 & 53.7 & 20.3 & 67.8 & 6.8 \\
\hline Norfentanyl & 76.7 & 14.9 & 79.1 & 9.2 & 99.8 & 5.9 & 82.6 & 7.8 & 76.5 & 14.5 & 79.8 & 7.3 \\
\hline \multicolumn{13}{|c|}{ SPE } \\
\hline Fentanyl & 69.7 & 5.0 & 64.1 & 5.5 & 88.6 & 8.0 & 62.4 & 8.4 & 61.7 & 8.4 & 63.7 & 8.5 \\
\hline 4-ANPP & 52.3 & 9.9 & 54.8 & 23.7 & 57.9 & 12.0 & 46.4 & 23.3 & 30.3 & 15.5 & 52.9 & 13.4 \\
\hline Norfentanyl & 100.8 & 5.3 & 126.2 & 5.1 & 112.5 & 5.9 & 106.7 & 2.4 & 113.5 & 7.7 & 134.6 & 5.7 \\
\hline
\end{tabular}

For postmortem analysis fentanyl is the primary target analyte and the QuEChERS extraction outperformed the LLE and SPE techniques for fentanyl with ME (80.4\%), RE (97.2\%), and PE (78.1\%). The RE of fentanyl using the QuEChERS extraction was $97.2 \%$ compared to the LLE and SPE methods with RE of $78.2 \%$ and $88.6 \%$ respectively. The QuEChERS extraction outperformed the LLE and SPE methods in repeatability with \%CV values of less than $15 \%$ for all ME, RE, and PE parameters whereas, LLE and SPE produced multiple above $15 \%$ and at least one above $20 \%$ which is above the threshold of acceptance. In comparison of the LLE and SPE techniques presented here, the SPE method produced better ME, RE, and PE results for fentanyl and norfentanyl than the LLE method. However, the LLE method generated better ME, RE, and PE for the analysis of 4-ANPP. Between the LLE and SPE techniques presented in this study 
there was not a clearly superior method. However, the QuEChERS technique performance was superior to both the LLE and SPE techniques presented in this study.

\subsubsection{Conclusions}

The QuEChERS extraction and subsequent LC-MS/MS method was fully validated for fentanyl, norfentanyl, and 4-ANPP in liver tissue. The bias for all three target analytes at all three concentrations did not exceed $\pm 20 \%$ with repeatability and reproducibility within the $\pm 20 \%$ threshold. The R2 values for the calibration curve of the three target analytes all exceeded 0.997 for the analytical measurable range (AMR) of $0.4-80 \mu \mathrm{g} / \mathrm{kg}$ for $4-A N P P$ and $0.5-100 \mu \mathrm{g} / \mathrm{kg}$ for fentanyl and norfentanyl. The QuEChERS technique had recoveries higher than $90.2 \%$, matrix effects greater than $80.4 \%$, and process efficiencies greater than $76.7 \%$ with $\% \mathrm{CV}$ values below $12.8 \%$ for all target analytes. The concentrations reported in this study, 56.6 to $462.3 \mu \mathrm{g} / \mathrm{kg}$ with a mean of $149.2 \mu \mathrm{g} / \mathrm{kg}(\mathrm{n}=10)$, are in an agreement with the concentrations reported by McIntyre, Luckenbill, and Palamalai.

Overall, the QuEChERS technique met all the acceptable criteria and was shown to be effective at extracting and quantitating fentanyl, norfentanyl, and 4-ANPP. The presented QuEChERS extraction outperformed the presented LLE and SPE techniques while reducing the amount of QuEChERS materials required for the extraction and the pretreatment process. Drug extraction from tissue requires a method that is sensitive, reduces the matrix effects, and allows for direct extraction from the tissue. The presented modified QuEChERS protocol meets all the requirements for liver extraction and reduces the risk of cross contamination in the homogenization process.

The above QuEChERS extraction was selected for expansion to include other fentanyl analogs and to validate for extraction of fentanyl and metabolites from insect tissues (larvae, pupae, empty puparium, and adult flies) presented in the next chapters. 


\subsection{Section 2: Expansion of Method to 34 Fentanyl Analogs}

\subsubsection{Overview}

Opioid addiction is a severe national crisis that affects public health, social and economic welfare. With the number of fentanyl overdose-related deaths rising at alarming rates, the interpretation of toxicological findings in these cases is a crucial component in medicolegal investigations. However, the vast number of emerging analogs combined with the potency of these drugs create several analytical challenges. It is necessary to develop a comprehensive strategy to facilitate the extraction of novel synthetic opioids (NSO) including fentanyl and its major metabolites from complex biological specimens such as liver tissue.

Objectives for this study include an expansion of a quick, easy, cheap, effective, rugged, and safe (QuEChERS) extraction and subsequent LC-MS/MS analysis, validation of the method following the American Academy of Forensic Sciences Standards Board (ASB) standard 036 requirements, and application to authentic liver specimens for 34 analytes including fentanyl, metabolites, and fentanyl analogs. With the emergence of NSO's, it is important for laboratories to have sensitive and effective extraction strategies for complex biological samples, a wide range of analytes to encompass potential emerging NSO's, and surveys of which specific NSO's are prevalent in the drug overdose community. This chapter presents an advancement in the combat of opioid epidemic by offering a fully validated protocol that complement the analytical toolkit in forensic toxicology laboratories and medical examiners offices. This section is reproduced in part with permission from Cox, J., Mathison, K., Ott, C., DelTondo, J., Kraner, J.C., DeCaprio, A.P., et al., Journal of Analytical Toxicology, 2021: 10.1093/jat/bkab009. 


\subsubsection{Introduction}

Fentanyl is a highly effective $\mu$-opioid agonist with a potency of $50-100$ times that of morphine that is used clinically as a schedule II drug and, like other opioids, has considerable addictive potential (91, 92). Illicitly manufactured fentanyl (IMF) and analogs have been abused individually and combined with other drugs like heroin often in counterfeit pill formulations (96-99). The structural scaffold of fentanyl can be modified in numerous ways to generate various versions of novel fentanyl analogs and this is exploited to produce IMF and analogs $(91,92,98)$. A deadly combination of intentional and unintentional misuse of fentanyl analogs and the high potency of the drug class has led to an increase in overdose deaths $(96,98,126)$. Novel synthetic opioids (NSOs) consist of different classes incorporating fentanyl analogs (4anilinopiperidine class), U-47700 type drugs (benzamide class), and MT-45 type drugs (piperazine derivatives) that all target the $\mu$-opioid receptors $(77,92,127)$. NSOs have a wide range of potencies with carfentanil as the most potent at up to 100 times more potent than fentanyl (91). Due to its potency, carfentanil has been involved in a multitude of overdose cases around the world in the past decade (110, 128-132).

Postmortem toxicological analysis in fentanyl related overdose is complicated by high potency of the drug, often resulting in low analyte concentrations and associations with toxicity, multi-drug use, novelty of emerging fentanyl analogs, and postmortem redistribution $(63,98,133,134)$. To overcome this adversity, toxicologists often examine multiple specimen matrices from different sources, including blood (femoral or heart), urine, vitreous fluid, liver tissue or other tissues $(63,134)$. For analysis of drugs that are susceptible to postmortem redistribution (PMR), liver tissue analysis is used to complement heart and femoral blood concentrations (64). As decomposition and the postmortem interval (PMI) progress, it has been proposed that tissues such as liver provide a more useful indication of antemortem drug concentrations compared to blood concentrations. As PMR occurs, the blood concentrations in the central cavities for drugs with a high volume of distribution $\left(V_{d}\right)$ often increases compared to those from 
peripheral areas, a phenomenon contributed to by passive diffusion of drugs from the surrounding organs $(117,118)$. Fentanyl is considered to have a high $V_{d}$ and fentanyl analogs that are structurally related are likely to exhibit similar characteristics and susceptibility to PMR (135).

Extraction of drugs from liver tissue is complex due to the high lipid and salt content naturally occurring in the matrix that can be further complicated as decomposition introduces byproducts from the putrefaction process as the PMI progresses (136-138). In 2003, a quick, easy, cheap, effective, rugged, and safe (QuEChERS) extraction method was established to detect pesticides in complex produce samples (79). To mediate the complications encountered with a postmortem tissue such as liver, a modified QuEChERS extraction can be used $(1,80,83)$. The two-part process of QuEChERS makes it an ideal procedure for tissue analysis due to a direct organic solvent extraction step followed by a dispersive solid phase extraction (d-SPE) step (4). This allows for direct solvent extraction from the tissue combined with the reduction of matrix effects, lipids, and other unwanted compounds via d-SPE $(1,4,80,83)$. QuEChERS extractions have been applied to drugs from liver tissue for benzodiazepines by Usui et al. and for fentanyl by this research group $(1,4)$. Application of QuEChERS to fentanyl analogs has been limited to acetyl fentanyl from urine in a fatal poisoning and to the knowledge of the authors there has not been an application of a QuEChERS extraction for fentanyl analogs from liver tissue (82).

Presented in this study is a development of a QuEChERS extraction and subsequent LC-MS/MS analysis, a validation of the method following the ASB standard 036 requirements, and application to 22 authentic liver specimens for 34 analytes including fentanyl, metabolites, and fentanyl analogs.

\subsubsection{Methods}

\subsubsection{Chemicals and Materials}

Drug standards: 2'-fluoro ortho-fluorofentanyl, ( \pm )-cis-3-methyl butyryl fentanyl $(\mathrm{HCl})$,

( \pm )-trans-3-methyl fentanyl ( $\mathrm{HCl}$ ), acetyl fentanyl, acryl fentanyl, acryl fentanyl-D5, benzodioxole fentanyl, benzyl fentanyl $(\mathrm{HCl})$, butyryl fentanyl $(\mathrm{HCl})$, butyryl norfentanyl $(\mathrm{HCl})$, cyclopentyl fentanyl $(\mathrm{HCl})$, 
despropionyl ortho-fluorofentanyl, furanyl fentanyl 3-furancarboxamide isomer $(\mathrm{HCl})$, meta-fluorofentanyl $(\mathrm{HCl})$, methoxyacetyl fentanyl, para-chlorofentanyl $(\mathrm{HCl})$, para-chloroisobutyryl fentanyl $(\mathrm{HCl})$, para-fluoro acrylfentanyl, para-fluoroisobutyryl fentanyl (FIBF), para-methoxyfentanyl $(\mathrm{HCl})$, tetrahydrofuran fentanyl $(\mathrm{HCl})$, thienyl fentanyl $(\mathrm{HCl})$, thiofentanyl $(\mathrm{HCl}), \mathrm{U}-47700, \mathrm{U}-47700-\mathrm{D} 6$, valeryl fentanyl, valeryl fentanyl-D5, a-methyl acetyl fentanyl $(\mathrm{HCl})$, $\alpha$-methyl butyryl fentanyl $(\mathrm{HCl})$, $\alpha$-methyl thiofentanyl $(\mathrm{HCl})$ and $\beta$-hydroxythiofentanyl $(\mathrm{HCl})$ were obtained from Cayman Chemical (Ann Arbor, MI) and were reconstituted or diluted to concentrations of $10 \mu \mathrm{g} / \mathrm{mL}$ or $100 \mu \mathrm{g} / \mathrm{mL}$ in methanol. Drug standards: 4-ANPP, carfentanil oxalate, fentanyl, furanyl fentanyl $(\mathrm{HCl})$, norfentanyl oxalate, cyclopropyl fentanyl, carfentanil-D5, fentanyl-D5, norfentanyl-D5, 4-ANPP-D5 and acetyl fentanyl-D5 were obtained from Cerilliant (Round Rock, TX) at concentrations of $1 \mathrm{mg} / \mathrm{mL}$ or $100 \mu \mathrm{g} / \mathrm{mL}$ in methanol. Optima $^{\circledR}$ liquid chromatography-mass spectrometry (LC-MS) grade methanol, acetonitrile, formic acid and ammonium formate were purchased from Thermo Fisher Scientific (Fair Lawn, NJ). All water was purified using a Direct- $Q^{\circledR} 3$ UV Water Purification System (Darmstadt, Germany). The QuEChERS original extraction salt packets (containing $6 \mathrm{~g}$ magnesium sulfate and $1.5 \mathrm{~g}$ sodium chloride) and $2 \mathrm{~mL}$ dispersiveSPE tubes (containing $25 \mathrm{mg}$ of primary secondary amine (PSA), $25 \mathrm{mg}$ end-capped octadecylsilane (C18EC) and $150 \mathrm{mg}$ magnesium sulfate) were purchased from Agilent Technologies. A Mixer Mill MM 200 was utilized for homogenization was obtained from Retsch ${ }^{\circledR}$ (Haan, Germany). Steel zinc plated BB balls (0.177 cal, $4.5 \mathrm{~mm}$ ) were obtained from Daisy (Rogers, AR).

Human liver specimens used in method development and validation were received from West Virginia University (WVU) Human Gift Registry $(n=5)$ from specimens received May 2019 to July 2019 and the Office of the Chief Medical Examiner Office (OCME) in Charleston, West Virginia $(n=17)$ from cases throughout 2019. Quantitative analysis was performed on authentic human liver specimens provided from both the suspected fentanyl-related deaths investigated by the Office of the Chief Medical Examiner Office (OCME) in Charleston, West Virginia and the West Virginia Human Gift Registry. The specimens 
from the West Virginia Human Gift Registry were not suspected of fentanyl related overdose deaths. Blank human liver specimens $(n=10)$ were also received from the West Virginia Human Gift Registry and were tested for the presence of fentanyl analogs before use. These liver specimens were used for the validation of the method as individual samples and equal portions were homogenized with a blender for the pooled specimens $(n=10)$. All samples were stored at $-20^{\circ} \mathrm{C}$ prior to analysis for approximately 4 to 10 months. All specimens were de-identified from personal identifying information and this study was determined to be exempt from Institutional Review Board (IRB) review due to postmortem specimen use and lack of human subject involvement.

\subsubsection{LC-MS/MS}

The quantification method was performed on an Agilent 6470 Triple Quad LC-MS/MS system coupled with an Agilent 1290 Infinity II LC system. Chromatographic separation was achieved on a Zorbax Eclipse Plus C18 RRHD 3.0x100 mm, $1.8 \mu \mathrm{m}$ column with $0.1 \%$ formic acid and $5 \mathrm{mM}$ ammonium formate in water (mobile phase $\mathrm{A}$ ) and $0.1 \%$ formic acid in methanol (mobile phase B). Data acquisition was performed in dynamic multiple reaction monitoring (dMRM) mode with positive ESI with a principal dMRM transition for quantitation and a qualifier dMRM transition for each analyte. A gradient elution at a flow rate of $0.3 \mathrm{~mL} / \mathrm{min}$ was run as follows: initial hold for $1 \mathrm{~min}$ at $5 \%$ mobile phase $B$ with a ramp to $60 \%$ mobile phase B for $1 \mathrm{~min}$, followed by a ramp to $70 \%$ B in 4 mins, followed by a ramp to $80 \%$ for 1 min, followed by a ramp to $95 \%$ for $30 \mathrm{~s}$ with a hold of $30 \mathrm{~s}$, and finally a $2.5 \mathrm{~min}$ post run for re-equilibrium at $5 \% \mathrm{~B}$.

The MS source parameters were performed as follows: gas temperature $325^{\circ} \mathrm{C}$; gas flow $9 \mathrm{~L} / \mathrm{min}$; nebulizer 45 psi; sheath gas flow $9 \mathrm{~L} / \mathrm{min}$; capillary voltage $3500 \mathrm{~V}$; and charging voltage $500 \mathrm{~V}$. Optimization of the data acquisition parameters was performed utilizing Agilent MassHunter Optimization software version B.08.02 in MRM mode to select the optimal precursor ions with corresponding 
fragmentor voltage and selection of product ions with associated collision energies for each transition. The optimized parameters are listed in Table 3.10.

The quantitation of analytes was performed using Agilent MassHunter Quantitative software version B.08.00. Relative responses were calculated using the absolute response of the drug in relation to response of the internal standard and plotted against drug concentrations.

The LC-MS/MS analysis was validated following the American Academy of Forensic Sciences Standards Board (ASB) standard 036 requirements (104). Evaluated parameters include interference studies, ionization suppression/enhancement, dilution integrity, limits of quantitation, linearity, processed sample stability, bias, and precision. 
Table 3.10: MS parameters for the 6470 Agilent system.

\begin{tabular}{|c|c|c|c|c|c|}
\hline Compound & Tran & nsition $^{a}$ & $\begin{array}{c}\text { Fragmentor Energy } \\
(\mathrm{V})\end{array}$ & $\begin{array}{c}\text { Collision } \\
\text { Energy (V) } \\
\end{array}$ & Internal Standard \\
\hline \multirow{2}{*}{ 2'-fluoro ortho-Fluorofentanyl } & 373.2 & $\rightarrow 206$ & 106 & 28 & \multirow{2}{*}{ D5-Acryl Fentanyl } \\
\hline & 373.2 & $\rightarrow 123$ & 106 & 48 & \\
\hline \multirow{2}{*}{ 4-ANPP } & 281.2 & $\rightarrow 188$ & 122 & 16 & \multirow{2}{*}{ D5-4-ANPP } \\
\hline & 281.2 & $\rightarrow \quad 105$ & 122 & 36 & \\
\hline \multirow{2}{*}{$\alpha$-Methyl Acetyl Fentanyl (3) } & 337.2 & $\rightarrow 91$ & 144 & 56 & \multirow{2}{*}{ D5-Acetyl Fentanyl } \\
\hline & 337.2 & $\rightarrow 119$ & 144 & 28 & \\
\hline \multirow{2}{*}{$\alpha$-Methyl Butyryl Fentanyl (11) } & 365.3 & $\rightarrow \quad 202.1$ & 122 & 24 & \multirow{2}{*}{ D5-Fentanyl } \\
\hline & 365.3 & $\rightarrow 91$ & 122 & 60 & \\
\hline \multirow{2}{*}{$\alpha$-Methyl Thiofentanyl } & 357.2 & $\rightarrow 97$ & 124 & 60 & \multirow{2}{*}{ D5-Valeryl Fentanyl } \\
\hline & 357.2 & $\rightarrow \quad 125$ & 124 & 32 & \\
\hline \multirow{2}{*}{$\beta$-Hydroxythiofentanyl } & 359.2 & $\rightarrow 111$ & 124 & 44 & \multirow{2}{*}{ D5-Fentanyl } \\
\hline & 359.2 & $\rightarrow 97$ & 124 & 52 & \\
\hline Acetyl Fentanyl (2) & 323.2 & $\rightarrow 188$ & 98 & 24 & D5-Acetvl Fentanyl \\
\hline 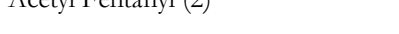 & 323.2 & $\rightarrow \quad 105$ & 98 & 44 & 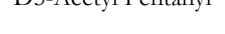 \\
\hline & 335.2 & $\rightarrow \quad 105$ & 94 & 44 & \\
\hline Acryl Fentanyl & 335.2 & $\rightarrow \quad 188$ & 94 & 24 & Do-Acryl Fentanyl \\
\hline & 429.2 & $\rightarrow \quad 149$ & 144 & 36 & D5-Acetyl Fentanyl \\
\hline Benzodioxole Fentanyl & 429.2 & $\rightarrow 105$ & 144 & 56 & Do-Acetyl Fentanyl \\
\hline Benzyl Fentanyl (5) & 323.2 & $\rightarrow 91$ & 124 & 48 & D5-4_ANPP \\
\hline Denzyн rentanyi (0) & 323.2 & $\rightarrow \quad 174.1$ & 124 & 20 & D)-4-ANPT \\
\hline Butyryl Fentanyl (10) & 351.2 & $\rightarrow \quad 105$ & 144 & 44 & D5-Valervl Fentanyl \\
\hline Dutyryi I critanyi (I0) & 351.2 & $\rightarrow \quad 188.1$ & 144 & 28 & DS-vaieryi \\
\hline & 247.2 & $\rightarrow 84.1$ & 100 & 20 & \\
\hline Butyryl Norfentanyl & 247.2 & $\rightarrow \quad 55.1$ & 100 & 48 & Do-Norfentanyl \\
\hline & 395.2 & $\rightarrow 335.1$ & 136 & 16 & D5_Carfentanil \\
\hline Carfentanil (/) & 395.2 & $\rightarrow 113$ & 136 & 36 & Do-Cartentanil \\
\hline Cis 3-Metbyl Buturyl Fentanyl (12) & 365.3 & $\rightarrow 105$ & 142 & 48 & D5-4-ANPP \\
\hline Cls-5-Metnyl Dutyryi Fentanyl (12) & 365.3 & $\rightarrow \quad 202.1$ & 142 & 24 & 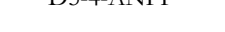 \\
\hline & 377.3 & $\rightarrow 188.1$ & 144 & 28 & D5-Valeryl Fentanyl \\
\hline Cyclopentyl Fentanyl & 377.3 & $\rightarrow \quad 105$ & 144 & 48 & Do-valeryl Fentanyl \\
\hline Cyclopropyl Fentanyl & 349.2 & $\rightarrow 105$ & 124 & 52 & D5-Fentanyl \\
\hline & 349.2 & $\rightarrow 77$ & 124 & 104 & D5-Fentanyl \\
\hline Despropionyl ortho-Fluorofentanyl & 299.2 & $\rightarrow 105$ & 104 & 36 & D5-Acryl Fentanyl \\
\hline Despropionyl ortho-Fluorofentanyl & 299.2 & $\rightarrow 77$ & 104 & 84 & Do-Acryl Fentanyl \\
\hline & 337.2 & $\rightarrow 105$ & 146 & 48 & D5-Fentanyl \\
\hline Fentanyl (0) & 337.2 & $\rightarrow 188$ & 146 & 24 & Do-rentanyr \\
\hline FIBF & 369.2 & $\rightarrow 188$ & 92 & 28 & D5-Acrvl Fentanyl \\
\hline & 369.2 & $\rightarrow 105$ & 92 & 48 & \\
\hline & 375.2 & $\rightarrow 188$ & 146 & 24 & D5-4_ANPP \\
\hline Furanyl Fentanyl & 375.2 & $\rightarrow 105$ & 146 & 48 & DS-4-ANPP \\
\hline Furanyl Fentanyl 3- & 375.2 & $\rightarrow 188.1$ & 124 & 28 & D5-4-ANPP \\
\hline furancarboxamide isomer & 375.2 & $\rightarrow \quad 105$ & 124 & 48 & D5-4-ANPP \\
\hline & 355.2 & $\rightarrow \quad 188.1$ & 144 & 28 & D5-Acryl Fentanyl \\
\hline Meta-Fluororentanyi & 355.2 & $\rightarrow \quad 105$ & 144 & 44 & \\
\hline Methoxy Acetyl Fentanyl & 353.2 & $\rightarrow 188$ & 92 & 24 & D5-4-ANPP \\
\hline 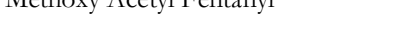 & 353.2 & $\rightarrow \quad 105$ & 92 & 44 & \\
\hline Norfentanyl (1) & 233.2 & $\rightarrow 84$ & 107 & 20 & D5-Norfentanyl \\
\hline Norfentanyl (1) & 233.2 & $\rightarrow \quad 55.1$ & 107 & 44 & DS-1Norientanyi \\
\hline Para_Chlorofentanyl & 371.2 & $\rightarrow 188.1$ & 124 & 28 & D5-Acrul Fentanyl \\
\hline Para-Chlorofentanyl & 371.2 & $\rightarrow \quad 105$ & 124 & 48 & Do-Acryl Fentanyl \\
\hline Para-Chloroisobutvryl Fentanvl & 385.2 & $\rightarrow \quad 188.1$ & 144 & 28 & D5-4-ANPP \\
\hline & 385.2 & $\rightarrow 105$ & 144 & 48 & \\
\hline Para-Fluoro Acryl Fentanyl & 353.2 & $\rightarrow 188.1$ & 124 & 24 & D5-Acrel Fentanyl \\
\hline Para-Fluoro Acryl Fentanyl & 353.2 & $\rightarrow 105$ & 124 & 48 & Do-Acryl Fentanyl \\
\hline & 367.2 & $\rightarrow 188.1$ & 124 & 28 & D5-Fentanyl \\
\hline Para-Methoxyfentanyl & 367.2 & $\rightarrow \quad 105$ & 124 & 48 & Do-Fentany \\
\hline Tetrahudrofuran Fentanyl & 379.2 & $\rightarrow 188.1$ & 124 & 24 & D5-Fentanyl \\
\hline 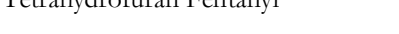 & 379.2 & $\rightarrow \quad 105$ & 124 & 48 & 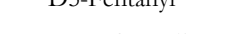 \\
\hline & 329.2 & $\rightarrow 97$ & 124 & 36 & D5-Carfentanil \\
\hline I hienyl Fentanyl (4) & 329.2 & $\rightarrow \quad 53.1$ & 124 & 92 & \\
\hline Thiofentanyl & 343.2 & $\rightarrow \quad 194.1$ & 124 & 24 & D5-4-ANPP \\
\hline Iniorentanyl & 343.2 & $\rightarrow 111$ & 124 & 44 & DS-4-ANNPP \\
\hline Trans-3-metbyl Fentanyl (8) & 351.2 & $\rightarrow \quad 202.1$ & 144 & 24 & D5-Fentanyl \\
\hline 1 rans-3-methyl Fentanyl (8) & 351.2 & $\rightarrow 105$ & 144 & 44 & Do-Fentanyl \\
\hline U-47700 (9) & 329.1 & $\rightarrow 173$ & 86 & 36 & D6-U-47700 \\
\hline $0-4 / 700(9)$ & 329.1 & $\rightarrow \quad 145$ & 86 & 64 & $D 0-U-4 / / 00$ \\
\hline Valervl Fentanyl (13) & 365.3 & $\rightarrow 105$ & 100 & 48 & D5-Valervl Fentanyl \\
\hline & 365.3 & $\rightarrow \quad 188$ & 100 & 28 & Do-Valeryl Fentanyl \\
\hline
\end{tabular}

Bold transition indicates quantifier transition; Numbers next to compounds relate to Figure 1 


\subsubsection{Sample Preparation}

The amount of sample required for the QuEChERS extraction was reduced to $0.1 \mathrm{~g}$ while limiting the risk of cross contamination by homogenizing the sample in a single reaction tube (4). The apparatus utilized steel beads and a bead beater type homogenizer allowing for homogenization of tissue while avoiding cross contamination risks associated with bladed devices (1). Liver tissue is often fatty in nature with many lipids and a modified QuEChERS protocol was selected to reduce the impact of the fatty nature of liver tissue $(137,138)$.

The QuEChERS extraction presented below is an adaptation from previous work from Cox et al. with expanded application to the 34 fentanyl analogs in this panel (4). Tissue samples weighing $0.1 \mathrm{~g}$ were placed in a $1.5 \mathrm{~mL}$ centrifuge tube, followed by $400 \mu \mathrm{L}$ deionized water, $100 \mu \mathrm{L}$ internal standard mix (IS), and $700 \mu \mathrm{L}$ acetonitrile, along with 3 steel beads. The tubes were vortexed, and $0.2 \mathrm{~g}$ QuEChERS original extraction salt was added to the tubes. The samples were then vortexed, mixed with a mixer mill for 3 min, centrifuged for $5 \mathrm{~min}$, and the supernatant was transferred to a QuEChERS dispersive tube. The tubes were vortexed and centrifuged again for 5 min. The supernatant was transferred to a new $1.5 \mathrm{~mL}$ centrifuge tube for dry down under a nitrogen stream at $50^{\circ} \mathrm{C}$ to dryness. The samples were reconstituted with $100 \mu \mathrm{L}$ of methanol and transferred to LC-MS/MS vials for analysis (4). The internal standard mix (IS) consisted of eight deuterated compounds: acryl fentanyl-D5, carfentanil-D5, fentanyl-D5, norfentanyl-D5, U-47700-D6, valeryl fentanyl-D5, 4-ANPP-D5 and acetyl fentanyl-D5 at a concentration of $25 \mathrm{ng} / \mathrm{mL}$.

\subsubsection{Validation}

\subsection{Calibration model}

Aliquots of $0.1 \mathrm{~g}$ of pooled human liver were spiked with $10 \mu \mathrm{L}$ of corresponding solutions to create calibration samples. Group A calibration solutions were created at $0.5,1,5,10,50$, and $100 \mu \mathrm{g} / \mathrm{kg}$ for 2-fluoro ortho-fluorofentanyl, acryl fentanyl, fentanyl, FIBF, furanyl fentanyl, norfentanyl, U-47700 and valeryl fentanyl. Group B calibration solutions were created at $0.1,0.5,1,5,10,50$ and $100 \mu \mathrm{g} / \mathrm{kg}$ for acetyl fentanyl, carfentanil and methoxy acetyl fentanyl. Group C calibration solutions were created at 
$0.25,0.5,2.5,5,25$, and $50 \mu \mathrm{g} / \mathrm{kg}$ for $\beta$-hydroxythiofentanyl, cis-3-methyl butyryl fentanyl, cyclopentyl fentanyl, cyclopropyl fentanyl and despropionyl ortho-fluorofentanyl. Group D calibration solutions were created at $0.1,0.2,1,2,10$ and $20 \mu \mathrm{g} / \mathrm{kg}$ for $\alpha$-methyl acetyl fentanyl, $\alpha$-methyl butyryl fentanyl, $\alpha$-methyl thiofentanyl, benzodioxole fentanyl, meta-fluorofentanyl, para-fluoro acryl fentanyl, paramethoxyfentanyl, tetrahydrofuran fentanyl, thiofentanyl and trans-3-methyl fentanyl. Group E calibration solutions were created at $0.05,0.1,0.5,1,5$ and $10 \mu \mathrm{g} / \mathrm{kg}$ for 4-ANPP, benzyl fentanyl, butyryl fentanyl, butyryl norfentanyl, furanyl fentanyl 3-furancarboxamide isomer, para-chlorofentanyl, parachloroisobutyryl fentanyl and thienyl fentanyl. Five replicates at each concentration were analyzed and regression lines were generated using Agilent MassHunter software using a weight of $(1 / x)$. A stock solution containing a mix of 2-fluoro ortho-fluorofentanyl ( $1 \mathrm{ng} / \mu \mathrm{L})$, 4-ANPP $(0.1 \mathrm{ng} / \mu \mathrm{L})$, acetyl Fentanyl (1 ng/ $\mu \mathrm{L})$, acryl Fentanyl (1 ng/ $\mu \mathrm{L}), \alpha$-methyl acetyl fentanyl $(0.2 \mathrm{ng} / \mu \mathrm{L}), \alpha$-methyl butyryl fentanyl $(0.2$ $\mathrm{ng} / \mu \mathrm{L})$, $\alpha$-methyl thiofentanyl $(0.2 \mathrm{ng} / \mu \mathrm{L})$, benzodioxole fentanyl $(0.2 \mathrm{ng} / \mu \mathrm{L})$, benzyl fentanyl $(0.1 \mathrm{ng} / \mu \mathrm{L})$, $\beta$-hydroxythiofentanyl $(0.5 \mathrm{ng} / \mu \mathrm{L})$, butyryl fentanyl $(0.1 \mathrm{ng} / \mu \mathrm{L})$, butyryl norfentanyl $(0.1 \mathrm{ng} / \mu \mathrm{L})$, carfentanil (1 $\mathrm{ng} / \mu \mathrm{L})$, cis-3-methyl butyryl fentanyl (0.5 $\mathrm{ng} / \mu \mathrm{L})$, cyclopentyl fentanyl (0.5 $\mathrm{ng} / \mu \mathrm{L})$, cyclopropyl fentanyl (0.5 ng/ $\mu \mathrm{L})$, despropionyl ortho-fluorofentanyl $(0.5 \mathrm{ng} / \mu \mathrm{L})$, fentanyl $(1 \mathrm{ng} / \mu \mathrm{L})$, FIBF (1 $\mathrm{ng} / \mu \mathrm{L})$, furanyl fentanyl (1 ng/ $\mu \mathrm{L})$, furanyl fentanyl 3-furancarboxamide isomer $(0.1 \mathrm{ng} / \mu \mathrm{L})$, metafluorofentanyl $(0.2 \mathrm{ng} / \mu \mathrm{L})$, methoxy acetyl fentanyl $(1 \mathrm{ng} / \mu \mathrm{L})$, norfentanyl $(1 \mathrm{ng} / \mu \mathrm{L})$, para-chlorofentanyl (0.1 ng/ $\mu \mathrm{L})$, para-chloroisobutyryl fentanyl $(0.1 \mathrm{ng} / \mu \mathrm{L})$, para-fluoro acryl fentanyl $(0.2 \mathrm{ng} / \mu \mathrm{L})$, paramethoxyfentanyl $(0.2 \mathrm{ng} / \mu \mathrm{L})$, tetrahydrofuran fentanyl $(0.2 \mathrm{ng} / \mu \mathrm{L})$, thienyl fentanyl $(0.1 \mathrm{ng} / \mu \mathrm{L})$, thiofentanyl $(0.2 \mathrm{ng} / \mu \mathrm{L})$, trans-3-methyl fentanyl $(0.2 \mathrm{ng} / \mu \mathrm{L}), \mathrm{U}-47700(1 \mathrm{ng} / \mu \mathrm{L})$, and valeryl fentanyl (1 $\mathrm{ng} / \mu \mathrm{L}$ ) was used as the highest calibrator and serial diluted for the remaining calibrators in the calibration curve. 


\subsection{Interference studies}

Potential interferences were evaluated using three separate approaches: evaluating blank matrix effects, interferences from stable-isotope internal standards, and potential interferences from commonly encountered analytes. Blank matrix samples from 10 individual sources of human liver were extracted without the addition of internal standard to demonstrate any matrix interferences. To evaluate any interferences from internal standards or interferences from standards to internal standards; blank matrix was spiked with internal standard only and an additional blank matrix replicate was spiked with at the highest calibration concentration with no internal standard added. The evaluation of potential interferences from other drug classes was performed by the analysis of blank liver spiked with a mix of 31 compounds incorporating other opioids, stimulants, depressants, synthetic cannabinoids, synthetic cathinones, and supplements shown in Table 3.11. Chromatographic analysis of these compounds was performed using Agilent MassHunter Qualitative Analysis software version B.08.00. 
Table 3. 11: List of 31 compounds evaluated for potential interferences.

\begin{tabular}{|c|c|c|c|}
\hline Class & Compound & Class & Compound \\
\hline \multirow[t]{6}{*}{ Opioids } & 6-Acetylmorphine & Synthetic Cannabinoids & JWH-018 \\
\hline & Oxycodone & & JWH-073 \\
\hline & Hydrocodone & & XLR-11 \\
\hline & Buprenorphine & & AB-FUBINACA \\
\hline & Norbuprenorphine & & AB-PINACA \\
\hline & Ethylmorphine & & MAM2201 \\
\hline \multirow[t]{2}{*}{ Opiates } & Morphine & Stimulants & Amphetamine \\
\hline & Codeine & & Methamphetamine \\
\hline \multirow[t]{9}{*}{ Supplements } & 1S,2R (+)-Ephedrine & & Cocaine \\
\hline & Methylphenidate & Synthetic Cathinones & $\begin{array}{c}\text { Alpha- } \\
\text { Pyrrolidinopentiophenone }(\alpha- \\
\text { PVP) }\end{array}$ \\
\hline & Sibutramine & & $\begin{array}{l}\text { 3,4-Methylenedioxy } \\
\text { Pyrovalerone }\end{array}$ \\
\hline & & & (MDPV) \\
\hline & Caffeine & Cannabinoids & Delta-9 THC (THC) \\
\hline & Synephrine & & Cannabidiol (CBD) \\
\hline & Octopamine & Other Alkaloids & Mitragynine \\
\hline & Methylhexanamine & & \\
\hline & (DMAA) & & \\
\hline
\end{tabular}

\subsection{Ionization suppression/enhancement}

Ionization suppression or enhancement was evaluated at two concentrations, low and high, for 10 replicates at each concentration following the ASB Standard 036 requirements (104). A desired percent ionization suppression or enhancement is $\pm 25 \%$ with a percent relative standard deviation (\%CV) not exceeding $20 \%$. Using a post-extraction addition approach, analyte peak areas of neat standards (Set 1 ) are compared to matrix samples fortified with neat standards after extraction (Set 2). Set 1 consisted of neat standards prepared at 1 (low) and 10 (high) $\mu \mathrm{g} / \mathrm{kg}$ and injected 6 times to establish a mean peak area for each concentration. Set 2 consisted of ten $0.1 \mathrm{~g}$ aliquots of individual human livers in duplicate, for a low $(n=10)$ and high $(n=10)$ concentration, extracted and spiked with the low and high concentration 
respectively. The average area of each set $(-X)$ was used to calculate the suppression or enhancement effect at each concentration for each analyte is as follows:

$$
\text { Ionization suppression or enhancement }(\%)=\left(\frac{\underline{X} \text { Area of set } 2}{\underline{X} \text { Area of } \operatorname{set} 1}-1\right) * 100
$$

\subsubsection{Bias and precision}

For bias and precision calculations, a blank pooled matrix of human liver $(n=10)$ was spiked and analyzed at low, med, and high concentrations for the 34 target analytes in triplicate over five days ( $n=15)$. Bias was calculated as a percent deviation of the mean from the theoretical concentration. Precision was evaluated by percent relative standard deviation (\%CV) of the mean at each concentration. The criteria for acceptable bias and precision (\%CV) was set at $\pm 20 \%$.

\subsection{Calibration model}

Aliquots of $0.1 \mathrm{~g}$ of pooled human liver were spiked with $10 \mu \mathrm{L}$ of corresponding solutions to create calibration samples. Group A calibration solutions were created at $0.5,1,5,10,50$, and $100 \mu \mathrm{g} / \mathrm{kg}$ for 2-fluoro ortho-fluorofentanyl, acryl fentanyl, fentanyl, FIBF, furanyl fentanyl, norfentanyl, U-47700 and valeryl fentanyl. Group B calibration solutions were created at $0.1,0.5,1,5,10,50$ and $100 \mu \mathrm{g} / \mathrm{kg}$ for acetyl fentanyl, carfentanil and methoxy acetyl fentanyl. Group C calibration solutions were created at $0.25,0.5,2.5,5,25$, and $50 \mu \mathrm{g} / \mathrm{kg}$ for $\beta$-hydroxythiofentanyl, cis-3-methyl butyryl fentanyl, cyclopentyl fentanyl, cyclopropyl fentanyl and despropionyl ortho-fluorofentanyl. Group D calibration solutions were created at $0.1,0.2,1,2,10$ and $20 \mu \mathrm{g} / \mathrm{kg}$ for $\alpha$-methyl acetyl fentanyl, $\alpha$-methyl butyryl fentanyl, $\alpha$-methyl thiofentanyl, benzodioxole fentanyl, meta-fluorofentanyl, para-fluoro acryl fentanyl, paramethoxyfentanyl, tetrahydrofuran fentanyl, thiofentanyl and trans-3-methyl fentanyl. Group E calibration solutions were created at $0.05,0.1,0.5,1,5$ and $10 \mu \mathrm{g} / \mathrm{kg}$ for 4-ANPP, benzyl fentanyl, butyryl fentanyl, butyryl norfentanyl, furanyl fentanyl 3-furancarboxamide isomer, para-chlorofentanyl, parachloroisobutyryl fentanyl and thienyl fentanyl. Five replicates at each concentration were analyzed and regression lines were generated using Agilent MassHunter software using a weight of $(1 / x)$. The highest 
calibrator concentration contained a mix of 2-fluoro ortho-fluorofentanyl (1 ng/ $\mu \mathrm{L}), 4$-ANPP $(0.1 \mathrm{ng} / \mu \mathrm{L})$, acetyl Fentanyl ( $1 \mathrm{ng} / \mu \mathrm{L})$, acryl Fentanyl (1 ng/ $\mu \mathrm{L}), \alpha$-methyl acetyl fentanyl $(0.2 \mathrm{ng} / \mu \mathrm{L}), \alpha$-methyl butyryl fentanyl ( $0.2 \mathrm{ng} / \mu \mathrm{L}), \alpha$-methyl thiofentanyl $(0.2 \mathrm{ng} / \mu \mathrm{L})$, benzodioxole fentanyl $(0.2 \mathrm{ng} / \mu \mathrm{L})$, benzyl fentanyl (0.1 ng/ $\mu \mathrm{L}), \beta$-hydroxythiofentanyl $(0.5 \mathrm{ng} / \mu \mathrm{L})$, butyryl fentanyl $(0.1 \mathrm{ng} / \mu \mathrm{L})$, butyryl norfentanyl $(0.1$

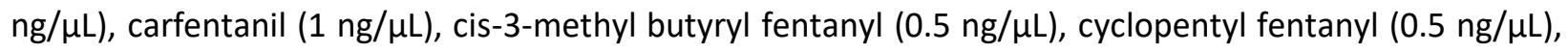
cyclopropyl fentanyl $(0.5 \mathrm{ng} / \mu \mathrm{L})$, despropionyl ortho-fluorofentanyl $(0.5 \mathrm{ng} / \mu \mathrm{L})$, fentanyl $(1 \mathrm{ng} / \mu \mathrm{L})$, FIBF (1 ng/ $\mu \mathrm{L})$, furanyl fentanyl $(1 \mathrm{ng} / \mu \mathrm{L})$, furanyl fentanyl 3-furancarboxamide isomer $(0.1 \mathrm{ng} / \mu \mathrm{L})$, metafluorofentanyl ( $0.2 \mathrm{ng} / \mu \mathrm{L})$, methoxy acetyl fentanyl ( $1 \mathrm{ng} / \mu \mathrm{L})$, norfentanyl $(1 \mathrm{ng} / \mu \mathrm{L})$, para-chlorofentanyl (0.1 ng/ $\mu \mathrm{L})$, para-chloroisobutyryl fentanyl $(0.1 \mathrm{ng} / \mu \mathrm{L})$, para-fluoro acryl fentanyl $(0.2 \mathrm{ng} / \mu \mathrm{L})$, paramethoxyfentanyl $(0.2 \mathrm{ng} / \mu \mathrm{L})$, tetrahydrofuran fentanyl $(0.2 \mathrm{ng} / \mu \mathrm{L})$, thienyl fentanyl $(0.1 \mathrm{ng} / \mu \mathrm{L})$, thiofentanyl $(0.2 \mathrm{ng} / \mu \mathrm{L})$, trans-3-methyl fentanyl $(0.2 \mathrm{ng} / \mu \mathrm{L}), \mathrm{U}-47700(1 \mathrm{ng} / \mu \mathrm{L})$, and valeryl fentanyl (1 $\mathrm{ng} / \mu \mathrm{L})$.

\subsubsection{Limits}

To evaluate the limit of detection (LOD) and lower limit of quantitation (LLOQ), aliquots of blank liver were spiked with concentrations at the lowest non-zero calibrator. Triplicates of unique blank matrix sources spiked at the lowest non-zero calibrator were analyzed over 3 days ( $n=9)$. Bias and precision (\%CV) for LLOQ was considered acceptable at $\pm 20 \%$. For LOD to be considered acceptable, signal to noise $(\mathrm{s} / \mathrm{n}$ ) ratios were to be above 3.3 and qualifier ratios within $\pm 20 \%$ to achieve chromatographic acceptance.

\subsubsection{Carryover and dilution integrity}

To evaluate carryover, blank matrix samples were injected directly after injections of samples 3 times greater than the highest calibrator using a triplicate analysis. Carryover is considered not significant if present below $10 \%$ of the lowest calibrator (LLOQ). To evaluate dilution integrity, a human liver sample in triplicate was spiked to 3 times the highest calibrator and the extract was diluted 1:5 with an extract of 
blank matrix with internal standard (106). The process was repeated over a period of 5 runs $(n=15)$ and the criteria for acceptable bias and precision (\%CV) was set at $\pm 20 \%$.

\subsubsection{Processed sample stability}

To determine the stability of processed samples in the autosampler $\left(25^{\circ} \mathrm{C}\right)$, aliquots of human liver were spiked at low $(5 \mu \mathrm{g} / \mathrm{kg})$ and high $(50 \mu \mathrm{g} / \mathrm{kg})$ concentrations followed by extraction. Triplicate analysis at time frames: $0,24,48$, and 72 hours were performed for each concentration. Average peak area was calculated for each triplicate and compared to time zero. An acceptable bias threshold was set at $\pm 20 \%$.

\subsubsection{Recovery, matrix effects and process efficiency}

To calculate matrix effects (ME), recovery of the extraction (RE), and process efficiency (PE) three formulas were utilized. Variables in the formulas represent the mean peak areas for the neat solution (A), the mean peak areas for the standards spiked after extraction (B), and the mean peak areas for the standards spiked before extraction (C)(107). Recovery, matrix effects, and process efficiency were evaluated using pooled human liver tissue $(n=10)$. Low and high concentrations were analyzed at ten replicates ( $n=10)$. A desired matrix effect is between $75 \%$ and $125 \%$ and the desired recovery is between $80 \%$ and $120 \%$ with a percent relative standard deviation (\%CV) not exceeding $20 \%$.

$$
\begin{aligned}
& M E(\%)=\frac{B}{A} * 100 \\
& R E(\%)=\frac{C}{B} * 100 \\
& P E(\%)=\frac{C}{A} * 100
\end{aligned}
$$




\subsubsection{Proof of applicability}

Liver specimens from 22 authentic postmortem cases provided by the OCME were submitted for analysis. Samples were analyzed using the QuEChERS extraction protocol presented above to display the methods ability to quantify the target analytes from the human liver matrix.

\subsubsection{Results and Discussion}

\subsubsection{Calibration model}

Five replicates at six different concentrations were analyzed and linear regression lines were generated using Agilent MassHunter software using a weight of $(1 / x)$. The analytical measurement range (AMR) is listed in Table 5 for each analyte. The R2 values for all the calibration curves exceeded 0.99 and the residuals for each set of 5 replicates were plotted and no discernable pattern was identified indicating a random dispersion. This allows for linear analysis of the 34 analytes utilizing a weight of $(1 / x)$.

\subsubsection{Interference studies}

Blank matrix samples from 10 individual sources of human liver were extracted without the addition of internal standard to determine potential matrix interferences. Chromatographic analysis of these compounds was performed using Agilent MassHunter Qualitative Analysis software version B.08.00. No interferences from the blank matrices were observed.

No interfering peaks from the high standards were observed for the internal standards and no interfering peaks were observed from the internal standards for the target analytes. The evaluation of commonly encountered interferences from other drug classes was performed by the analysis of blank liver spiked with a mix of 31 compounds incorporating other opioids, stimulants, depressants, synthetic cannabinoids, synthetic cathinones, and supplements shown in Table 2. No interferences from the 31 compounds were observed.

Isobaric compounds were identified, and individual standards were injected to ensure that chromatographic separation occurred. Figure 3.8 displays the chromatogram of 11 isobaric compounds in the method. The numbers in figure 3.8 correspond to the numbers in table 3.10 for the dMRM transitions 
monitored. The analytes themselves did not cause interferences with each other. The concentration of each inject standard was $500 \mathrm{ng} / \mathrm{mL}$.

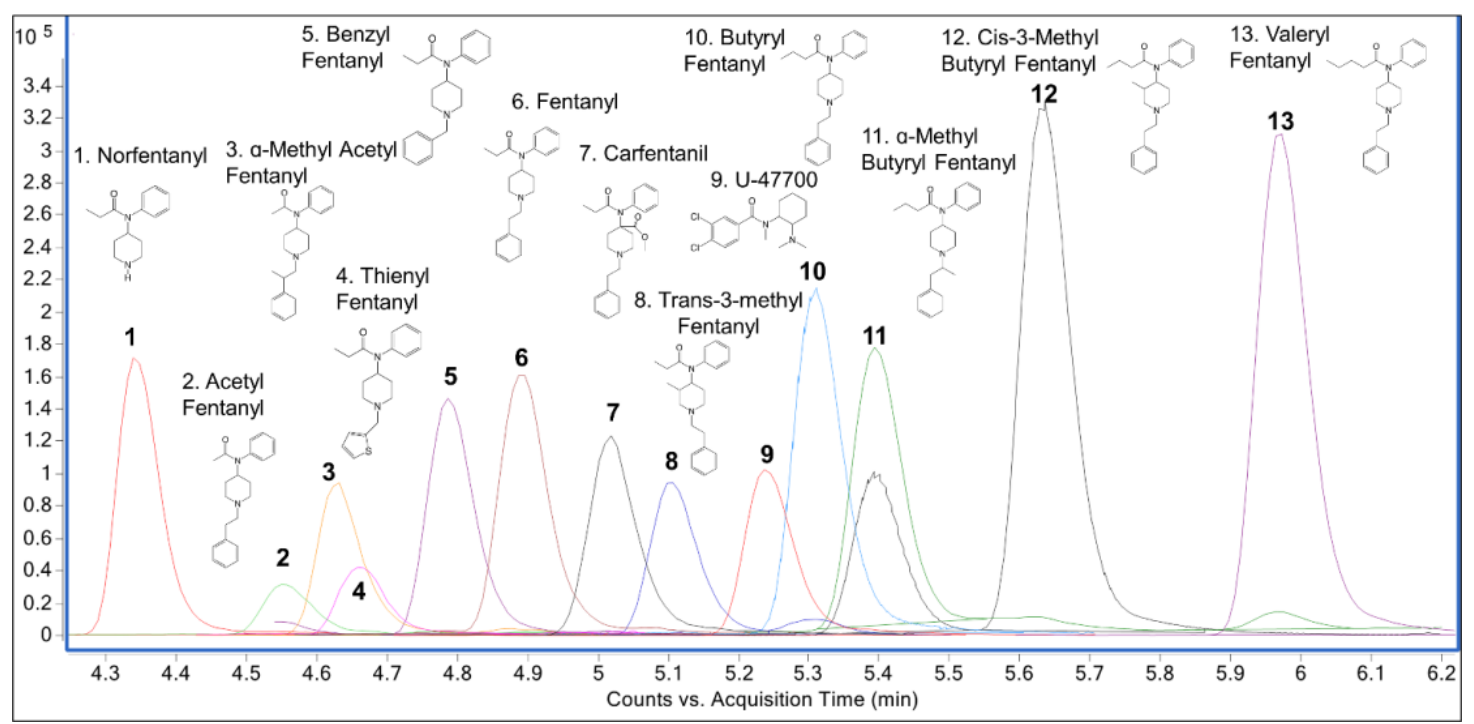

Figure 3.8: Chromatogram of the 11 isobaric fentanyl analogs with norfentanyl and carfentanil for reference. Numbers 1-13 in the figure correspond to Table 1.

\subsubsection{Ionization suppression/enhancement}

lonization suppression or enhancement was evaluated at a low and high concentration for 10 replicates at each concentration following the ASB Standard 036 requirements. The percent ionization suppression or enhancement and \%CV for each target compound is presented in Table 3.12. The criteria for acceptance is $\pm 25 \%$ ionization suppression or enhancement with a $\%$ CV not exceeding $20 \%$. All 34 analytes were within the criteria with the low concentration ranging from $-10.2 \%$ to $23.7 \%$ ionization suppression or enhancement and the high concentration ranging from $-7.1 \%$ to $11.0 \%$ ionization suppression or enhancement. The only compound to exceed $\pm 15 \%$ for suppression or enhancement was butyryl norfentanyl at the low concentration at $23.7 \%$ enhancement. This was within the acceptable range but noted as being considerably higher than any other analyte. A reason for this could be attributed to the compound being a metabolite and therefore did not have a deuterated internal standard that was similar enough in structure. 


\subsubsection{Bias and precision}

For bias and precision calculations, a blank pooled matrix of human liver $(n=10)$ was spiked and analyzed at low, medium, and high concentrations for the 34 target analytes in triplicate over five days $(n=15)$ and the results are displayed in Table 3.13. The bias for all 34 fentanyl analogs were within $\pm 20 \%$ and the $\% \mathrm{CV}$ did not exceed $20 \%$. None of the 34 fentanyl analogs had a bias that exceeded $\pm 10 \%$ for any of the low, medium, or high concentrations. The bias for the low ranged between $-9.2 \%$ and $4.7 \%$. The bias for the medium ranged between $0.5 \%$ and $6.9 \%$ and the high ranged between $-6.9 \%$ and $1.9 \%$. Benzyl fentanyl had a higher bias for low and medium concentrations however it did not exceed $\pm 10 \%$. The addition of a deuterated internal standard for this compound could help reduce the bias for this fentanyl analog. The between run and within run precision for all 34 fentanyl analogs were within the acceptable criteria. Benzodioxole fentanyl had the highest within run \%CV at the low concentration and had the highest \%CV for any analyte at any concentration but was still within the acceptable limit.

\subsubsection{Limits}

Triplicates of unique blank matrix sources spiked at the lowest non-zero calibrator were analyzed over 3 days ( $n=9)$. Bias and precision ( $\%$ CV) for LLOQ was considered acceptable at $\pm 20 \%$ and are displayed in Table 3.14. The bias for the LLOQs ranged from $-7.8 \%$ to $12.0 \%$ and within the acceptable criteria. For LOD to be considered acceptable, signal to noise $(s / n)$ ratios were to be above 3.3 and qualifier ratios within $\pm 20 \%$ to achieve chromatographic acceptance. LOD values for each of the 34 fentanyl analogs are listed in Table 3.14 below.

\subsubsection{Carryover and dilution integrity}

To evaluate carryover, blank matrix samples were injected directly after injections of samples with concentrations three times greater than the highest calibrator using a triplicate analysis. No significant carryover of analytes was detected in the blank matrix following the injection of samples with concentrations three times greater than the highest calibrator. Table 3.15 displays the values of bias and precision calculated for each analyte analyzed for dilution integrity. For the 34 analytes, the bias for the 
dilution integrity samples did not exceed $\pm 10.1 \%$ or a precision greater than $12.5 \% \mathrm{CV}$. Overall, the bias for all analytes were negative indicating a lower than anticipated value. Even though the bias is deemed acceptable this should be monitored in the future. The least dilution integrity bias was calculated for 4ANPP (-2.6\%) and the highest was para-fluoro acryl fentanyl (-10.1\%). 
Table 3.12: Ionization suppression/enhancement.

\begin{tabular}{|c|c|c|c|c|}
\hline \multirow{3}{*}{ Compound } & \multicolumn{4}{|c|}{ Ionization Suppression/Enhancement } \\
\hline & \multicolumn{2}{|c|}{ Low } & \multicolumn{2}{|c|}{ High } \\
\hline & Mean \% & $\% \mathrm{CV}$ & Mean \% & $\% \mathrm{CV}$ \\
\hline 2'-fluoro ortho-Fluorofentanyla & 7.5 & 9.8 & 2.9 & 5.3 \\
\hline 4-ANPPd & 10.7 & 11.2 & 2.9 & 4.7 \\
\hline$\alpha$-Methyl Acetyl Fentanylc & 2.2 & 10.3 & 6.8 & 8.5 \\
\hline$\alpha$-Methyl Butyryl Fentanylc & 6.1 & 10.4 & 8.3 & 8.7 \\
\hline$\alpha$-Methyl Thiofentanylc & -3.5 & 10.9 & 5.0 & 6.5 \\
\hline$\beta$-Hydroxythiofentanyl ${ }^{b}$ & -10.2 & 13.5 & -0.3 & 6.6 \\
\hline Acetyl Fentanyl ${ }^{\mathrm{a}}$ & 7.3 & 10.3 & 9.3 & 7.5 \\
\hline Acryl Fentanyla & 0.5 & 6.1 & 3.9 & 5.7 \\
\hline Benzodioxole Fentanylc & 14.5 & 10.8 & 7.6 & 8.7 \\
\hline Benzyl Fentanyld & -0.4 & 13.3 & 6.5 & 9.1 \\
\hline Butyryl Fentanyld & 9.0 & 8.7 & 4.3 & 7.5 \\
\hline Butyryl Norfentanyld & 23.7 & 17.4 & 10.9 & 13.2 \\
\hline Carfentanila $^{a}$ & 5.8 & 7.0 & -0.1 & 3.8 \\
\hline Cis-3-Methyl Butyryl Fentanylb & 2.7 & 12.2 & 0.4 & 5.3 \\
\hline Cyclopentyl Fentanyl ${ }^{\mathrm{b}}$ & 1.7 & 7.6 & 3.7 & 4.8 \\
\hline Cyclopropyl Fentanyl ${ }^{\mathrm{b}}$ & 5.3 & 9.5 & 5.0 & 4.9 \\
\hline $\begin{array}{l}\text { Despropionyl ortho- } \\
\text { Fluorofentanyl }{ }^{b}\end{array}$ & 0.5 & 14.5 & 2.3 & 8.0 \\
\hline Fentanyla $^{\mathrm{a}}$ & 3.4 & 7.5 & 0.1 & 4.8 \\
\hline $\mathrm{FIBFa}$ & 8.8 & 14.0 & 11.0 & 17.6 \\
\hline Furanyl Fentanyla & 7.9 & 8.0 & 3.4 & 6.5 \\
\hline $\begin{array}{l}\text { Furanyl Fentanyl 3- } \\
\text { furancarboxamide isomer }^{\mathrm{d}}\end{array}$ & 11.2 & 10.5 & 4.3 & 9.2 \\
\hline Meta-Fluorofentanylc & 2.3 & 16.2 & 2.3 & 4.7 \\
\hline Methoxy Acetyl Fentanyla & -4.4 & 15.8 & -7.1 & 15.9 \\
\hline Norfentanyla & 11.9 & 11.1 & 4.2 & 5.6 \\
\hline Para-Chlorofentanyld & 8.9 & 10.8 & 6.0 & 11.2 \\
\hline $\begin{array}{l}\text { Para-Chloroisobutyryl } \\
\text { Fentanyld }\end{array}$ & 14.5 & 16.3 & 10.2 & 19.2 \\
\hline Para-Fluoro Acryl Fentanylc & 7.9 & 10.9 & 3.3 & 6.0 \\
\hline Para-Methoxyfentanylc & 3.6 & 9.6 & 5.5 & 6.5 \\
\hline Tetrahydrofuran Fentanylc & 5.1 & 8.8 & 2.4 & 7.4 \\
\hline Thienyl Fentanyld & -0.6 & 9.4 & 4.1 & 12.4 \\
\hline Thiofentanylc & -8.5 & 16.6 & 0.2 & 5.5 \\
\hline Trans-3-methyl Fentanylc & 1.5 & 6.1 & 4.5 & 7.7 \\
\hline $\mathrm{U}-47700^{\mathrm{a}}$ & 6.9 & 4.3 & 3.5 & 2.5 \\
\hline Valeryl Fentanyla & 3.5 & 6.8 & 4.0 & 8.3 \\
\hline
\end{tabular}

${ }^{a}$ Low concentration at $1 \mu \mathrm{g} / \mathrm{kg}$, and high concentration at $50 \mu \mathrm{g} / \mathrm{kg}$

b Low concentration at $0.5 \mu \mathrm{g} / \mathrm{kg}$, and high concentration at $25 \mu \mathrm{g} / \mathrm{kg}$

c Low concentration at $0.2 \mu \mathrm{g} / \mathrm{kg}$, and high concentration at $10 \mu \mathrm{g} / \mathrm{kg}$

d Low concentration at $0.1 \mu \mathrm{g} / \mathrm{kg}$, and high concentration at $5 \mu \mathrm{g} / \mathrm{kg}$ 
Table 3.13:: Bias and precision data for the 34 fentanyl analogs.

\begin{tabular}{|c|c|c|c|c|c|c|c|c|c|}
\hline \multirow{2}{*}{ Compound } & \multicolumn{3}{|c|}{ Bias $(\%)$} & \multicolumn{3}{|c|}{ Between Run $(\% \mathrm{CV})$} & \multicolumn{3}{|c|}{ Within Run (\%CV) } \\
\hline & LOW & MED & HIGH & LOW & MED & HIGH & LOW & MED & $\mathrm{HIGH}$ \\
\hline $\begin{array}{l}\text { 2'-fluoro ortho- } \\
\text { Fluorofentanyla }\end{array}$ & -3.2 & 4.7 & -0.2 & 6.1 & 5.4 & 6.9 & 5.5 & 4.8 & 9.2 \\
\hline 4-ANPPd & -1.2 & 3.2 & -2.9 & 11.0 & 9.6 & 9.1 & 9.6 & 9.6 & 11.0 \\
\hline$\alpha$-Methyl Acetyl Fentanylc & 2.3 & 1.6 & 0.3 & 16.8 & 7.4 & 7.6 & 12.5 & 9.6 & 13.2 \\
\hline $\begin{array}{l}\alpha \text {-Methyl Butyryl } \\
\text { Fentanylc }\end{array}$ & -0.9 & 0.5 & -6.9 & 5.5 & 5.6 & 16.5 & 6.4 & 5.8 & 12.2 \\
\hline$\alpha$-Methyl Thiofentanylc & 4.6 & 4.3 & 0.8 & 13.9 & 4.3 & 5.5 & 11.5 & 4.8 & 5.7 \\
\hline$\beta$-Hydroxythiofentanyl & -3.0 & 5.1 & -0.3 & 17.5 & 7.2 & 7.8 & 16.9 & 10.5 & 8.1 \\
\hline Acetyl Fentanyla & -7.8 & 5.3 & 0.3 & 8.3 & 6.8 & 8.8 & 10.4 & 10.3 & 14.4 \\
\hline Acryl Fentanyla & -0.1 & 6.0 & -0.6 & 5.5 & 6.8 & 7.1 & 7.3 & 5.8 & 9.1 \\
\hline Benzodioxole Fentanylc & -3.2 & 4.6 & 0.8 & 13.5 & 6.7 & 6.5 & 19.3 & 9.2 & 8.9 \\
\hline Benzyl Fentanyld & -9.2 & 6.5 & 0.3 & 13.1 & 7.9 & 7.7 & 15.2 & 11.9 & 8.9 \\
\hline Butyryl Fentanyld & -2.3 & 5.8 & 0.3 & 8.4 & 7.6 & 7.0 & 13.1 & 8.0 & 7.6 \\
\hline Butyryl Norfentanyld & -7.7 & 4.4 & 1.9 & 16.6 & 7.1 & 8.7 & 12.3 & 4.9 & 6.8 \\
\hline Carfentanila & -7.6 & 4.6 & -0.1 & 8.6 & 7.2 & 6.9 & 9.9 & 7.7 & 9.6 \\
\hline $\begin{array}{l}\text { Cis-3-Methyl Butyryl } \\
\text { Fentanylb }\end{array}$ & -6.0 & 3.2 & -2.0 & 7.8 & 4.6 & 7.0 & 9.1 & 5.7 & 10.1 \\
\hline Cyclopentyl Fentanyl ${ }^{\mathrm{b}}$ & 0.4 & 3.8 & -1.6 & 6.3 & 5.4 & 7.2 & 5.4 & 6.4 & 7.4 \\
\hline Cyclopropyl Fentanyl ${ }^{\mathrm{b}}$ & -0.8 & 5.4 & -1.0 & 6.4 & 6.2 & 6.2 & 10.1 & 6.0 & 7.0 \\
\hline $\begin{array}{l}\text { Despropionyl ortho- } \\
\text { Fluorofentanylb }\end{array}$ & -2.1 & 4.6 & -1.2 & 8.4 & 6.2 & 6.8 & 9.2 & 6.7 & 8.9 \\
\hline Fentanyl $^{\mathrm{a}}$ & -4.3 & 6.0 & -0.8 & 7.1 & 7.5 & 7.4 & 10.6 & 6.3 & 7.8 \\
\hline FIBFa & -0.7 & 4.1 & -0.8 & 5.6 & 5.3 & 6.6 & 5.2 & 4.0 & 9.2 \\
\hline Furanyl Fentanyla & -2.9 & 4.0 & 1.4 & 7.4 & 5.1 & 6.4 & 9.1 & 7.4 & 10.2 \\
\hline $\begin{array}{l}\text { Furanyl Fentanyl 3- } \\
\text { furancarboxamide isomerd }\end{array}$ & -1.3 & 6.9 & 1.8 & 11.6 & 6.1 & 8.1 & 17.7 & 7.3 & 10.9 \\
\hline Meta-Fluorofentanylc & -0.7 & 3.5 & -0.5 & 7.0 & 6.6 & 7.3 & 11.0 & 8.2 & 10.7 \\
\hline Methoxy Acetyl Fentanyla & -4.0 & 3.8 & -1.6 & 9.6 & 7.5 & 7.9 & 8.2 & 9.3 & 11.0 \\
\hline Norfentanyla & -0.2 & 1.2 & 1.6 & 10.5 & 8.7 & 5.8 & 11.7 & 8.4 & 8.0 \\
\hline Para-Chlorofentanyld & -4.3 & 4.0 & -0.1 & 11.5 & 7.4 & 8.2 & 8.4 & 9.9 & 8.9 \\
\hline $\begin{array}{l}\text { Para-Chloroisobutyryl } \\
\text { Fentanyld }\end{array}$ & -3.0 & 3.1 & -3.3 & 11.3 & 7.0 & 8.4 & 15.4 & 10.1 & 11.1 \\
\hline $\begin{array}{l}\text { Para-Fluoro Acryl } \\
\text { Fentanylc }\end{array}$ & 4.2 & 4.1 & -1.1 & 5.0 & 9.6 & 7.6 & 6.6 & 15.7 & 10.4 \\
\hline Para-Methoxyfentanylc & 3.0 & 5.8 & -1.1 & 9.3 & 6.1 & 7.5 & 16.7 & 7.0 & 9.4 \\
\hline Tetrahydrofuran Fentanylc & 2.2 & 4.7 & 0.1 & 6.2 & 4.3 & 7.3 & 9.8 & 5.3 & 6.7 \\
\hline Thienyl Fentanyld & -3.1 & 5.4 & -0.1 & 9.6 & 8.2 & 6.6 & 6.4 & 4.9 & 9.8 \\
\hline Thiofentanylc & 1.5 & 3.4 & -1.9 & 10.8 & 4.4 & 7.5 & 15.3 & 7.2 & 10.7 \\
\hline Trans-3-Methyl Fentanylc & 4.7 & 4.2 & -1.5 & 5.1 & 6.1 & 8.0 & 7.9 & 5.7 & 9.1 \\
\hline U-47700a & 3.2 & 6.1 & 0.3 & 7.4 & 6.6 & 7.8 & 9.0 & 6.5 & 8.7 \\
\hline Valeryl Fentanyla & -3.7 & 4.2 & 0.0 & 5.7 & 6.2 & 7.0 & 8.9 & 10.1 & 8.2 \\
\hline
\end{tabular}


Table 3.14: LOD, LLOQ, and AMR.

\begin{tabular}{|c|c|c|c|c|}
\hline \multirow{2}{*}{ Compound } & \multirow{2}{*}{$\begin{array}{l}\mathrm{LOD}^{\mathrm{a}} \\
(\mu \mathrm{g} / \mathrm{kg})\end{array}$} & \multicolumn{2}{|c|}{$\operatorname{LLOQ}^{\mathrm{b}}(\mathrm{n}=9)$} & \multirow{2}{*}{$\begin{array}{l}\mathrm{AMR}^{\mathrm{c}} \\
(\mu \mathrm{g} / \mathrm{kg})\end{array}$} \\
\hline & & Bias & $\% \mathrm{CV}$ & \\
\hline $\begin{array}{l}\text { 2'-fluoro ortho- } \\
\text { Fluorofentanyl }\end{array}$ & 0.5 & 1.3 & 9.5 & $0.5-100$ \\
\hline 4-ANPP & 0.05 & 4.2 & 12.1 & $0.05-10$ \\
\hline$\alpha$-Methyl Acetyl fentanyl & 0.1 & -5.6 & 12.8 & $0.1-20$ \\
\hline$\alpha$-Methyl Butyryl Fentanyl & 0.1 & 14.1 & 15.6 & $0.1-20$ \\
\hline$\alpha$-Methyl Thiofentanyl & 0.1 & 1.2 & 16.2 & $0.1-20$ \\
\hline$\beta$-Hydroxythiofentanyl & 0.25 & -2.6 & 5.8 & $0.25-50$ \\
\hline Acetyl Fentanyl & 0.1 & -7.0 & 9.3 & $0.1-100$ \\
\hline Acryl Fentanyl & 0.5 & -0.4 & 10.3 & $0.5-100$ \\
\hline Benzodioxole Fentanyl & 0.1 & 3.8 & 16.4 & $0.1-20$ \\
\hline Benzyl Fentanyl & 0.05 & 3.5 & 12.2 & $0.05-10$ \\
\hline Butyryl Fentanyl & 0.05 & -4.1 & 6.1 & $0.05-10$ \\
\hline Butyryl Norfentanyl & 0.05 & -3.3 & 11.2 & $0.05-10$ \\
\hline Carfentanil & 0.1 & -2.4 & 2.7 & $0.1-100$ \\
\hline $\begin{array}{l}\text { Cis-3-Methyl Butyryl } \\
\text { Fentanyl }\end{array}$ & 0.25 & -0.6 & 10.5 & $0.25-50$ \\
\hline Cyclopentyl Fentanyl & 0.25 & -5.5 & 8.2 & $0.25-50$ \\
\hline Cyclopropyl Fentanyl & 0.25 & 1.5 & 8.8 & $0.25-50$ \\
\hline $\begin{array}{l}\text { Despropionyl ortho- } \\
\text { Fluorofentanyl }\end{array}$ & 0.25 & -7.8 & 7.7 & $0.25-50$ \\
\hline Fentanyl & 0.5 & -4.6 & 10.2 & $0.5-100$ \\
\hline FIBF & 0.5 & -0.1 & 9.8 & $0.5-100$ \\
\hline Furanyl Fentanyl & 0.5 & 12.0 & 12.9 & $0.5-100$ \\
\hline $\begin{array}{l}\text { Furanyl Fentanyl 3- } \\
\text { furancarboxamide isomer }\end{array}$ & 0.05 & 4.7 & 10.8 & $0.05-10$ \\
\hline Meta-Fluorofentanyl & 0.1 & -5.5 & 16.4 & $0.1-20$ \\
\hline Methoxy Acetyl Fentanyl & 0.1 & 3.9 & 8.9 & $0.1-100$ \\
\hline Norfentanyl & 0.5 & 6.1 & 8.8 & $0.5-100$ \\
\hline Para-Chlorofentanyl & 0.05 & -9.2 & 15.9 & $0.05-10$ \\
\hline $\begin{array}{l}\text { Para-Chloroisobutyryl } \\
\text { Fentanyl }\end{array}$ & 0.05 & -3.8 & 14.5 & $0.05-10$ \\
\hline $\begin{array}{l}\text { Para-Fluoro Acryl } \\
\text { Fentanyl }\end{array}$ & 0.1 & 7.3 & 9.8 & $0.1-20$ \\
\hline Para-Methoxyfentanyl & 0.1 & -6.2 & 10.4 & $0.1-20$ \\
\hline Tetrahydrofuran Fentanyl & 0.1 & 0.5 & 14.8 & $0.1-20$ \\
\hline Thienyl Fentanyl & 0.05 & -6.4 & 15.8 & $0.05-10$ \\
\hline Thiofentanyl & 0.1 & 4.9 & 12.1 & $0.1-20$ \\
\hline Trans-3-Methyl Fentanyl & 0.1 & 3.0 & 10.0 & $0.1-20$ \\
\hline U-47700 & 0.5 & 0.2 & 10.9 & $0.5-100$ \\
\hline Valeryl Fentanyl & 0.5 & -12.7 & 7.3 & $0.5-100$ \\
\hline
\end{tabular}

${ }^{\mathrm{a}}$ LOD (Limit of detection)

b LLOQ (Lower limit of quantitation)

c AMR (Analytical measurement range) 


\subsubsection{Processed sample stability}

Processed sample stability in the autosampler was evaluated in triplicate at low and high concentrations for four time intervals up to 72 hours. All 34 fentanyl analogs were stable beyond 48 hours however valeryl fentanyl was exceeded $20 \%$ after 72 hours at the low and high concentrations (Table 3.16).

Table 3.15: Dilution integrity of post extraction dilution at a 1:5 dilution $(n=15)$.

\begin{tabular}{|c|c|c|}
\hline Compound & Dilution Integrity (Bias \%) & $\% \mathrm{CV}$ \\
\hline 2'-fluoro ortho-Fluorofentanyla & -6.3 & 10.7 \\
\hline 4-ANPPd & -2.6 & 10.0 \\
\hline$\alpha$-Methyl Acetyl Fentanylc & -5.6 & 11.4 \\
\hline$\alpha$-Methyl Butyryl Fentanylc & -7.0 & 8.6 \\
\hline$\alpha$-Methyl Thiofentanylc & -6.0 & 11.8 \\
\hline$\beta$-Hydroxythiofentanylb & -8.0 & 10.3 \\
\hline Acetyl Fentanyl ${ }^{a}$ & -6.6 & 10.3 \\
\hline Acryl Fentanyla & -7.0 & 10.4 \\
\hline Benzodioxole Fentanylc & -6.1 & 11.4 \\
\hline Benzyl Fentanyld & -4.9 & 11.2 \\
\hline Butyryl Fentanyld & -7.3 & 11.2 \\
\hline Butyryl Norfentanyld & -3.4 & 11.2 \\
\hline Carfentanila $^{a}$ & -4.6 & 9.4 \\
\hline Cis-3-Methyl Butyryl Fentanylb & -5.6 & 9.1 \\
\hline Cyclopentyl Fentanyl ${ }^{\mathrm{b}}$ & -5.5 & 11.6 \\
\hline Cyclopropyl Fentanyl b & -8.3 & 8.3 \\
\hline Despropionyl ortho-Fluorofentanyl ${ }^{\mathrm{b}}$ & -7.1 & 8.8 \\
\hline Fentanyla & -7.6 & 10.2 \\
\hline FIBFa & -5.7 & 10.0 \\
\hline Furanyl Fentanyl ${ }^{\mathrm{a}}$ & -4.9 & 11.2 \\
\hline Furanyl Fentanyl 3-furancarboxamide isomer ${ }^{\mathrm{d}}$ & -6.6 & 11.9 \\
\hline Meta-Fluorofentanylc & -7.1 & 10.9 \\
\hline Methoxy Acetyl Fentanyla & -4.3 & 12.4 \\
\hline Norfentanyla $^{\mathrm{a}}$ & -3.6 & 11.7 \\
\hline Para-Chlorofentanyld & -6.5 & 11.9 \\
\hline Para-Chloroisobutyryl Fentanyld & -2.3 & 11.2 \\
\hline Para-Fluoro Acryl Fentanylc & -10.1 & 10.6 \\
\hline Para-Methoxyfentanylc & -7.3 & 9.2 \\
\hline Tetrahydrofuran Fentanylc & -7.9 & 11.1 \\
\hline Thienyl Fentanyld & -5.4 & 11.8 \\
\hline Thiofentanylc & -6.1 & 11.4 \\
\hline Trans-3-Methyl Fentanylc & -6.3 & 9.7 \\
\hline U-47700a & -7.4 & 12.5 \\
\hline Valeryl Fentanyl ${ }^{a}$ & -8.9 & 8.6 \\
\hline
\end{tabular}


Table 3.16: Stability at a low and high concentration for the 34 analytes for processed samples stored in the autosampler $\left(25^{\circ} \mathrm{C}\right)$

\begin{tabular}{|c|c|c|c|c|c|c|}
\hline \multirow{2}{*}{ Compound } & \multicolumn{3}{|c|}{ Low $\left(\% \Delta^{\mathrm{e}}\right)$} & \multicolumn{3}{|c|}{ High $\left(\% \Delta^{\mathrm{e}}\right)$} \\
\hline & $24 \mathrm{~h}$ & $48 \mathrm{~h}$ & $72 \mathrm{~h}$ & $24 \mathrm{~h}$ & $48 \mathrm{~h}$ & $72 \mathrm{~h}$ \\
\hline 2'-fluoro ortho-Fluorofentanyla & -0.3 & 2.7 & 7.1 & -0.4 & 1.9 & 2.4 \\
\hline 4-ANPPd & 7.1 & 12.0 & 8.7 & 0.6 & 2.7 & -0.5 \\
\hline$\alpha$-Methyl Acetyl ]Fentanylc & 0.7 & 5.0 & -0.2 & -2.5 & 4.6 & -0.6 \\
\hline$\alpha$-Methyl Butyryl Fentanylc & -0.3 & 4.6 & 4.6 & -1.1 & 3.4 & 1.0 \\
\hline$\alpha$-Methyl Thiofentanylc & 1.2 & 2.1 & -0.8 & -0.4 & 0.4 & -2.5 \\
\hline$\beta$-Hydroxythiofentanyl $\mathrm{l}^{\mathrm{b}}$ & 1.3 & 13.9 & 10.7 & -1.8 & 4.2 & 1.7 \\
\hline Acetyl Fentanyla & -1.2 & 4.6 & 2.8 & -2.3 & 1.8 & 0.9 \\
\hline Acryl Fentanyl ${ }^{a}$ & -0.1 & 2.2 & -0.1 & -3.0 & 1.7 & -2.2 \\
\hline Benzodioxole Fentanylc & -4.6 & 3.4 & 4.1 & -1.5 & 3.9 & 0.9 \\
\hline Benzyl Fentanyld & 1.6 & 8.1 & 2.0 & 0.2 & 0.7 & -2.1 \\
\hline Butyryl Fentanyld & 0.0 & 6.6 & 4.5 & -1.9 & 2.7 & -0.3 \\
\hline Butyryl Norfentanyld & 3.0 & 5.4 & -0.5 & 1.6 & 3.5 & -0.6 \\
\hline Carfentanila & -1.6 & 3.0 & 3.6 & -3.0 & 1.9 & -0.4 \\
\hline Cis-3-Methyl Butyryl Fentanylb & 4.0 & 7.5 & 3.4 & 1.4 & 2.8 & 2.2 \\
\hline Cyclopentyl Fentanyl ${ }^{\mathrm{b}}$ & -5.5 & 2.1 & 9.1 & -13.8 & -0.3 & 4.6 \\
\hline Cyclopropyl Fentanyl b & 1.1 & 0.2 & 3.0 & 0.5 & 1.9 & 0.2 \\
\hline Despropionyl ortho-Fluorofentanyl ${ }^{\mathrm{b}}$ & -6.7 & 2.6 & 2.0 & -12.1 & 0.0 & -1.0 \\
\hline Fentanyla & 0.4 & 4.5 & 1.8 & -1.7 & 3.3 & 0.9 \\
\hline FIBFa & 2.2 & 5.4 & 6.6 & 0.1 & 4.83 & 2.2 \\
\hline Furanyl Fentanyla & 0.3 & 2.8 & 0.2 & -1.2 & 2.5 & -0.2 \\
\hline Furanyl Fentanyl 3-furancarboxamide isomerd & -2.7 & 1.6 & -1.0 & -2.5 & 4.0 & -2.9 \\
\hline Meta-Fluorofentanylc & 2.2 & 4.6 & 2.7 & -2.5 & 2.7 & -1.0 \\
\hline Methoxy Acetyl Fentanyla & -0.8 & 3.0 & -0.3 & 1.7 & 4.7 & 0.8 \\
\hline Norfentanyla & 10.0 & 14.3 & 13.8 & 1.3 & 7.2 & 4.2 \\
\hline Para-Chlorofentanyld & -4.0 & 10.6 & 6.7 & -8.9 & 3.6 & -0.5 \\
\hline Para-Chloroisobutyryl Fentanyld & -3.6 & 13.5 & 5.6 & -5.2 & 9.6 & 4.6 \\
\hline Para-Fluoro Acryl Fentanylc & -2.7 & -3.8 & 4.0 & -0.3 & 1.4 & -2.2 \\
\hline Para-Methoxyfentanylc & -1.1 & 1.1 & 3.5 & -1.3 & -1.2 & -1.6 \\
\hline Tetrahydrofuran Fentanylc & -4.1 & 6.4 & -0.1 & -1.8 & 1.1 & 0.1 \\
\hline Thienyl Fentanyld & -7.5 & 2.9 & 0.9 & 0.7 & 2.9 & 0.6 \\
\hline
\end{tabular}




\begin{tabular}{|c|c|c|c|c|c|c|}
\hline Thiofentanylc & 0.6 & 0.2 & -2.0 & 0.1 & 0.7 & -2.8 \\
\hline Trans-3-methyl Fentanylc & -4.9 & 3.2 & 1.7 & -12.6 & -1.4 & -1.4 \\
\hline $\mathrm{U}-47700^{\mathrm{a}}$ & 0.9 & 7.1 & 2.5 & -1.6 & 2.6 & -1.3 \\
\hline Valeryl Fentanyla & -12.2 & -12.0 & 21.6 & -4.7 & 5.1 & 31.2 \\
\hline $\begin{array}{l}\text { a Low concentration at } 5 \mu \mathrm{g} / \mathrm{kg} \text {, } \\
\text { b Low concentration at } 2.5 \mu \mathrm{g} / \mathrm{k} \\
{ }^{\mathrm{c}} \text { Low concentration at } 1 \mu \mathrm{g} / \mathrm{kg} \text {, } \\
\text { d Low concentration at } 0.5 \mu \mathrm{g} / \mathrm{k}\end{array}$ & $\begin{array}{l}\text { at } 50 \mu \mathrm{g} \\
\mathrm{n} \text { at } 25 \\
\text { at } 10 \mu \mathrm{g} \\
\mathrm{n} \text { at } 5 \mu\end{array}$ & $\begin{array}{l}\mathrm{kg} \\
\mathrm{h} / \mathrm{kg} \\
\mathrm{kg} \\
\mathrm{kg}\end{array}$ & & & & \\
\hline
\end{tabular}

\subsubsection{Recovery, matrix effects and process efficiency}

The analysis of the recovery, matrix effects and process efficiency were calculated for each of the 34 fentanyl analogs at their respective concentrations listed in Table 3.17. The average matrix effects at low and high concentrations for the 34 fentanyl analogs were $99.2 \%$ and $98.3 \%$ respectively. The average recovery at low and high concentrations for the 34 fentanyl analogs were $101.1 \%$ and $104.4 \%$ respectively. The average process efficiencies for all 34 fentanyl analogs were at $100.2 \%$ and $102.6 \%$ for the low and high concentrations respectively.

\subsubsection{Proof of applicability}

Liver specimens from 22 authentic postmortem cases provided by the West Virginia OCME $(n=17)$ and WVU Human Gift Registry $(n=5)$ were submitted for analysis. The human specimens submitted by the OCME were from suspected drug related deaths, while the specimens from the WVU Human Gift Registry were not suspected of drug related deaths. The northeastern U.S. is heavily impacted by drug overdose deaths, containing the top six states with the highest age-adjusted mortality rates with West Virginia (51.5 per 100,000 standard population) having the highest rate (84). 
Table 3.17: Matrix effects, recovery, and process efficiency.

\begin{tabular}{|c|c|c|c|c|c|c|c|}
\hline Compound & & Matrix Effects (\%) & $\begin{array}{c}\mathrm{RSD}^{\mathrm{a}} \\
(\%)\end{array}$ & Recovery (\%) & $\begin{array}{c}\text { RSD }^{a} \\
(\%)\end{array}$ & Process Efficiency (\%) & $\begin{array}{c}\mathrm{RSD}^{\mathrm{a}} \\
(\%)\end{array}$ \\
\hline 2'-fluoro ortho- & Low $(1 \mu \mathrm{g} / \mathrm{kg})$ & 101.9 & 11.6 & 97.6 & 8.2 & 99.4 & 12.1 \\
\hline Fluorofentanyl & High $(50 \mu \mathrm{g} / \mathrm{kg})$ & 101.8 & 5.3 & 109.3 & 7.5 & 111.3 & 6.5 \\
\hline \multirow[t]{2}{*}{ 4-ANPP } & Low $(0.1 \mu \mathrm{g} / \mathrm{kg})$ & 108.4 & 13.5 & 97.5 & 8.7 & 105.7 & 13.4 \\
\hline & High $(5 \mu \mathrm{g} / \mathrm{kg})$ & 99 & 8 & 105.6 & 9.5 & 104.6 & 7.6 \\
\hline$\alpha$-Methyl Acetyl & Low $(0.1 \mu \mathrm{g} / \mathrm{kg})$ & 108.1 & 13.2 & 114.5 & 21.6 & 123.8 & 22.7 \\
\hline Fentanyl & High $(10 \mu \mathrm{g} / \mathrm{kg})$ & 97.1 & 8.3 & 102.5 & 10.2 & 99.5 & 8.6 \\
\hline$\alpha$-Methyl Butyryl & Low $(0.1 \mu \mathrm{g} / \mathrm{kg})$ & 97.9 & 12 & 95.2 & 10 & 93.2 & 13.2 \\
\hline Fentanyl & High $(10 \mu \mathrm{g} / \mathrm{kg})$ & 99.8 & 8.1 & 95.7 & 9.3 & 95.5 & 7.5 \\
\hline \multirow[t]{2}{*}{$\alpha$-Methyl Thiofentanyl } & Low $(0.1 \mu \mathrm{g} / \mathrm{kg})$ & 101.3 & 14 & 96.5 & 14.2 & 97.8 & 15.8 \\
\hline & High $(10 \mu \mathrm{g} / \mathrm{kg})$ & 99.2 & 8 & 97.3 & 8.7 & 96.5 & 6.7 \\
\hline \multirow[t]{2}{*}{$\beta$-Hydroxythiofentanyl } & Low $(0.1 \mu \mathrm{g} / \mathrm{kg})$ & 113.7 & 24.1 & 95.6 & 23.3 & 108.7 & 25.4 \\
\hline & High $(25 \mu \mathrm{g} / \mathrm{kg})$ & 92.6 & 8.5 & 96.8 & 9.4 & 89.7 & 7 \\
\hline \multirow[t]{2}{*}{ Acetyl Fentanyl } & Low $(1 \mu \mathrm{g} / \mathrm{kg})$ & 82.8 & 11.1 & 114.4 & 10.2 & 94.7 & 12.5 \\
\hline & High $(50 \mu \mathrm{g} / \mathrm{kg})$ & 93.9 & 8.2 & 124.5 & 10.5 & 117 & 7.6 \\
\hline \multirow[t]{2}{*}{ Acryl Fentanyl } & Low $(1 \mu \mathrm{g} / \mathrm{kg})$ & 100.8 & 9.7 & 97.9 & 6.2 & 98.6 & 9.9 \\
\hline & High $(50 \mu \mathrm{g} / \mathrm{kg})$ & 101.2 & 5 & 116.2 & 7 & 117.6 & 6 \\
\hline \multirow[t]{2}{*}{ Benzodioxole Fentanyl } & Low $(0.1 \mu \mathrm{g} / \mathrm{kg})$ & 105 & 11.6 & 111.7 & 14.1 & 117.3 & 16.5 \\
\hline & High $(10 \mu \mathrm{g} / \mathrm{kg})$ & 104 & 6.6 & 97.7 & 7.7 & 101.6 & 5.6 \\
\hline \multirow[t]{2}{*}{ Benzyl Fentanyl } & Low $(0.1 \mu \mathrm{g} / \mathrm{kg})$ & 96.4 & 11.6 & 107.3 & 16 & 103.4 & 18.4 \\
\hline & High $(5 \mu \mathrm{g} / \mathrm{kg})$ & 99.8 & 6.8 & 98.9 & 8 & 98.7 & 6.5 \\
\hline \multirow[t]{2}{*}{ Butyryl Fentanyl } & Low $(0.5 \mu \mathrm{g} / \mathrm{kg})$ & 99.4 & 9.5 & 99.4 & 6.2 & 98.8 & 10.1 \\
\hline & High $(5 \mu \mathrm{g} / \mathrm{kg})$ & 99 & 5.5 & 110.5 & 7.4 & 109.3 & 6.2 \\
\hline \multirow[t]{2}{*}{ Butyryl Norfentanyl } & Low $(0.5 \mu \mathrm{g} / \mathrm{kg})$ & 92.5 & 9.3 & 95.5 & 7.4 & 88.3 & 9.1 \\
\hline & High $(5 \mu \mathrm{g} / \mathrm{kg})$ & 97.8 & 3.8 & 104.4 & 7.5 & 102.1 & 6.9 \\
\hline \multirow[t]{2}{*}{ Carfentanil } & Low $(1 \mu \mathrm{g} / \mathrm{kg})$ & 103 & 9.7 & 97.8 & 7.4 & 100.7 & 10.3 \\
\hline & High $(50 \mu \mathrm{g} / \mathrm{kg})$ & 101.6 & 6.6 & 102.8 & 8.2 & 104.5 & 7.4 \\
\hline Cis-3-Methyl Butyryl & Low $(0.5 \mu \mathrm{g} / \mathrm{kg})$ & 82.7 & 11.5 & 102.7 & 11.5 & 85.0 & 10.9 \\
\hline Fentanyl & High $(25 \mu \mathrm{g} / \mathrm{kg})$ & 88.9 & 9.3 & 100 & 11.2 & 89.0 & 8.9 \\
\hline \multirow[t]{2}{*}{ Cyclopentyl Fentanyl } & Low $(0.5 \mu \mathrm{g} / \mathrm{kg})$ & 99.9 & 10.1 & 95.5 & 7.3 & 95.5 & 11.2 \\
\hline & High $(25 \mu \mathrm{g} / \mathrm{kg})$ & 98.9 & 7.5 & 97.4 & 8.6 & 96.4 & 6.7 \\
\hline Cyclopropyl Fentanyl & Low $(0.5 \mu \mathrm{g} / \mathrm{kg})$ & 99.2 & 10.8 & 99.5 & 8.3 & 98.8 & 12.5 \\
\hline & High $(25 \mu \mathrm{g} / \mathrm{kg})$ & 100 & 7.4 & 95.6 & 7.9 & 95.7 & 6.1 \\
\hline Despropionyl ortho- & Low $(0.5 \mu \mathrm{g} / \mathrm{kg})$ & 97.9 & 10.2 & 99.5 & 8.7 & 97.4 & 11.6 \\
\hline Fluorofentanyl & High $(25 \mu \mathrm{g} / \mathrm{kg})$ & 98.1 & 6.3 & 101.4 & 7.4 & 99.5 & 5.7 \\
\hline Fentanyl & Low $(1 \mu \mathrm{g} / \mathrm{kg})$ & 99.8 & 9.1 & 97.5 & 7 & 97.4 & 10 \\
\hline & High $(50 \mu \mathrm{g} / \mathrm{kg})$ & 100.1 & 5.1 & 112.6 & 6.7 & 112.6 & 5.8 \\
\hline FIBF & Low $(1 \mu \mathrm{g} / \mathrm{kg})$ & 101.7 & 10.4 & 95.9 & 6.2 & 97.5 & 10.6 \\
\hline & High $(50 \mu \mathrm{g} / \mathrm{kg})$ & 101.7 & 6.1 & 99.6 & 7.6 & 101.3 & 6.4 \\
\hline Furanyl Fentanyl & Low $(1 \mu \mathrm{g} / \mathrm{kg})$ & 102.9 & 9.4 & 101.8 & 6.2 & 104.8 & 9.6 \\
\hline & High $(50 \mu \mathrm{g} / \mathrm{kg})$ & 100.1 & 5.3 & 130.3 & 7.9 & 130.4 & 7.4 \\
\hline Furanyl Fentanyl 3- & Low $(0.1 \mu \mathrm{g} / \mathrm{kg})$ & 101.6 & 9.4 & 103.6 & 5.3 & 105.3 & 8.4 \\
\hline $\begin{array}{l}\text { furancarboxamide } \\
\text { isomer }\end{array}$ & High $(5 \mu \mathrm{g} / \mathrm{kg})$ & 99.9 & 5.7 & 127 & 8.3 & 126.8 & 7.6 \\
\hline Meta-Fluorofentanyl & Low $(0.1 \mu \mathrm{g} / \mathrm{kg})$ & 102.1 & 12.8 & 98.5 & 12.4 & 100.6 & 15.9 \\
\hline & High $(10 \mu \mathrm{g} / \mathrm{kg})$ & 98.4 & 7.5 & 95.6 & 9.1 & 94.1 & 7.3 \\
\hline Methoxy Acetyl & Low $(1 \mu \mathrm{g} / \mathrm{kg})$ & 76.8 & 11.4 & 106.1 & 9.4 & 81.5 & 11 \\
\hline Fentanyl & High $(50 \mu \mathrm{g} / \mathrm{kg})$ & 86.4 & 7 & 118 & 10.5 & 102 & 9.2 \\
\hline Norfentanyl & Low $(1 \mu \mathrm{g} / \mathrm{kg})$ & 110.4 & 13.5 & 97.8 & 14 & 108 & 11.4 \\
\hline & High $(50 \mu \mathrm{g} / \mathrm{kg})$ & 94.3 & 4.4 & 116.3 & 9.1 & 109.7 & 9.1 \\
\hline Para-Chlorofentanyl & Low $(0.1 \mu \mathrm{g} / \mathrm{kg})$ & 100 & 11.1 & 99.6 & 10.1 & 99.6 & 12.1 \\
\hline & High $(5 \mu \mathrm{g} / \mathrm{kg})$ & 99.3 & 7.3 & 96.2 & 8.4 & 95.5 & 6.7 \\
\hline Para-Chloroisobutyryl & Low $(0.1 \mu \mathrm{g} / \mathrm{kg})$ & 102.1 & 11.2 & 105.7 & 8 & 108 & 11.8 \\
\hline Fentanyl & High $(5 \mu \mathrm{g} / \mathrm{kg})$ & 100.5 & 6.8 & 105.8 & 9.6 & 106.4 & 8.7 \\
\hline Para-Fluoro Acryl & Low $(0.1 \mu \mathrm{g} / \mathrm{kg})$ & 94.4 & 21.2 & 104.3 & 18 & 98.4 & 19.5 \\
\hline Fentanyl & High $(10 \mu \mathrm{g} / \mathrm{kg})$ & 101.9 & 10 & 96.7 & 10.7 & 98.6 & 8.4 \\
\hline Para-Methoxyfentanyl & Low $(0.1 \mu \mathrm{g} / \mathrm{kg})$ & 95.7 & 14.2 & 102.3 & 14 & 97.9 & 14.6 \\
\hline & High $(10 \mu \mathrm{g} / \mathrm{kg})$ & 99.4 & 7.5 & 93.9 & 8.4 & 93.4 & 6.9 \\
\hline Tetrahydrofuran & Low $(0.1 \mu \mathrm{g} / \mathrm{kg})$ & 97.6 & 12.4 & 93.9 & 10.1 & 91.7 & 11.5 \\
\hline Fentanyl & High $(10 \mu \mathrm{g} / \mathrm{kg})$ & 96.3 & 7.2 & 95.6 & 7.8 & 92.1 & 6.1 \\
\hline Thienyl Fentanyl & Low $(0.1 \mu \mathrm{g} / \mathrm{kg})$ & 101 & 12.3 & 98.7 & 10.1 & 99.7 & 13.6 \\
\hline & High $(5 \mu \mathrm{g} / \mathrm{kg})$ & 99.8 & 7.8 & 100.3 & 8.2 & 100.1 & 6.9 \\
\hline Thiofentanyl & Low $(0.1 \mu \mathrm{g} / \mathrm{kg})$ & 97 & 25.2 & 121.5 & 20.6 & 117.9 & 22.2 \\
\hline & High $(10 \mu \mathrm{g} / \mathrm{kg})$ & 95.7 & 7.3 & 97.4 & 8.2 & 93.2 & 6.2 \\
\hline Trans-3-Methyl & Low $(0.1 \mu \mathrm{g} / \mathrm{kg})$ & 96.7 & 13.4 & 93.3 & 12.7 & 90.2 & 15.3 \\
\hline Fentanyl & High $(10 \mu \mathrm{g} / \mathrm{kg})$ & 98 & 6.5 & 95.8 & 7.7 & 93.9 & 6.5 \\
\hline U-47700 & Low $(1 \mu \mathrm{g} / \mathrm{kg})$ & 101.8 & 9.2 & 98.2 & 7.5 & 100 & 9.8 \\
\hline & High $(50 \mu \mathrm{g} / \mathrm{kg})$ & 99 & 7.1 & 98.2 & 8 & 97.2 & 6.4 \\
\hline Valeryl Fentanyl & Low $(1 \mu \mathrm{g} / \mathrm{kg})$ & 100.1 & 9.4 & 100.5 & 6.2 & 100.6 & 9.9 \\
\hline & High $(50 \mu \mathrm{g} / \mathrm{kg})$ & 99.6 & 5.4 & 114.1 & 7.2 & 113.7 & 6 \\
\hline
\end{tabular}


Of the 22 specimens, 17 contained fentanyl and metabolites plus at least one fentanyl analog. Three specimens were positive for only fentanyl and metabolites. Two specimens were only positive for FIBF as the only analyte present. The highest concentration for any fentanyl analog was $541 \mu \mathrm{g} / \mathrm{kg}$ for FIBF, which was the only target analyte detected in that sample. Nine of the 22 samples contained at least two fentanyl analogs in addition to fentanyl and metabolites. Three of the 22 samples contained three fentanyl analogs along with fentanyl and metabolites. The concentrations for fentanyl $(n=20)$ ranged between $3.6 \mu \mathrm{g} / \mathrm{kg}$ to $165 \mu \mathrm{g} / \mathrm{kg}$ with a mean of $54.7 \mu \mathrm{g} / \mathrm{kg}$. The fentanyl analog that was most encountered was methoxyacetyl fentanyl $(n=11)$ with a minimum concentration of $0.2 \mu \mathrm{g} / \mathrm{kg}$, a maximum of $4.6 \mu \mathrm{g} / \mathrm{kg}$, and a mean of $1.3 \mu \mathrm{g} / \mathrm{kg}$. Acetyl fentanyl was detected in six specimens with a mean concentration of $23.3 \mu \mathrm{g} / \mathrm{kg}$ and carfentanil was found in five specimens with a mean concentration of 8.4 $\mu \mathrm{g} / \mathrm{kg}$. Methoxyacetyl fentanyl and acetyl fentanyl have a potency of approximately 0.3 times that of fentanyl however carfentanil has a potency of 30 to 100 times that of fentanyl (91). Of the 22 specimens, three contained only fentanyl with metabolites and two contained only a fentanyl analog. This indicates that fentanyl was detected in combination with at least one fentanyl analog in a majority of the liver specimens. Due to the potency of fentanyl analogs and the various degrees of tolerance for different users, a wide range of concentrations encountered in case samples is not uncommon and have been reported in recent publications $(130,139)$.

All of the specimens from the WVU Human Gift Registry were positive for norfentanyl and fentanyl, with mean concentrations of $6.4 \mu \mathrm{g} / \mathrm{kg}$ (range 1.0 to $14.1 \mu \mathrm{g} / \mathrm{kg}$ ) and $30.5 \mu \mathrm{g} / \mathrm{kg}$ (range 3.9 to 117 ) respectively. All samples were positive for at minimum one target analyte from the 34 compounds and the results are shown in Table 3.18. For the 17 OCME samples, norfentanyl was detected in 11 and fentanyl in 15, with mean concentrations of $10 \mu \mathrm{g} / \mathrm{kg}$ (range 0.5 to $45 \mu \mathrm{g} / \mathrm{kg}$ ) and $62.8 \mu \mathrm{g} / \mathrm{kg}$ (range 3.6 to $165 \mu \mathrm{g} / \mathrm{kg}$ ) respectively. The concentrations of norfentanyl and fentanyl were substantially higher in the OCME liver 
specimens compared to those from the WVU Human Gift Registry, which is to be expected as the OCME specimens were from drug related overdose deaths while the others involved therapeutic use.

Table 3. 18: Authentic postmortem liver results.

\begin{tabular}{|c|c|c|c|c|c|c|}
\hline Compound & $\begin{array}{l}\text { \# positive } \\
\text { samples }\end{array}$ & $\begin{array}{c}\text { Mean } \\
(\mu g / k g)\end{array}$ & $\begin{array}{l}\text { Standard } \\
\text { Deviation }\end{array}$ & $\begin{array}{l}\text { Median } \\
(\mu g / \mathrm{kg})\end{array}$ & $\begin{array}{c}\text { Min } \\
(\mu g / \mathrm{kg})\end{array}$ & $\begin{array}{c}\mathrm{Max} \\
(\mu \mathrm{g} / \mathrm{kg})\end{array}$ \\
\hline Fentanyl & 20 & 54.7 & 48.8 & 51.2 & 3.6 & 164.9 \\
\hline Norfentanyl & 16 & 8.9 & 11.6 & 5.9 & 0.5 & 45 \\
\hline 4-ANPP & 15 & 8.9 & 8.9 & 6.9 & 0.9 & 33.6 \\
\hline $\begin{array}{l}\text { Methoxy Acetyl } \\
\text { Fentanyl }\end{array}$ & 11 & 1.3 & 1.4 & 0.9 & 0.2 & 4.6 \\
\hline Acetyl Fentanyl & 6 & 7.2 & 9.7 & 2.0 & 0.3 & 23.3 \\
\hline Carfentanil & 5 & 2.0 & 3.6 & 0.3 & 0.2 & 8.4 \\
\hline FIBF & 4 & 138.8 & 268.5 & 6.6 & 0.6 & 541.4 \\
\hline $\begin{array}{l}\alpha \text {-Methyl Acetyl } \\
\text { Fentanyl }\end{array}$ & 2 & 1.0 & - & 1.0 & 1.0 & 1.1 \\
\hline $\begin{array}{l}\text { Trans-3-methyl } \\
\text { Fentanyl }\end{array}$ & 1 & 32.4 & - & - & - & - \\
\hline Valeryl Fentanyl & 1 & 1.1 & - & - & - & - \\
\hline
\end{tabular}

Various NSOs have been studied in multiple matrices including blood, urine, hair, oral fluid, and other tissues however, the matrices most studied are blood or urine $(77,126,127,140-142)$. Recent publications on extractions from postmortem blood and tissue report percent ionization suppression for fentanyl analogs greater than $-20 \%$ while using traditional solid phase extraction (SPE) techniques that utilize organic solvents in $\mathrm{mL}$ volumes per sample $(133,142)$. The method developed in this study presented percent ionization suppression/enhancement numbers below $15 \%$ for most analytes while using amount of organic solvent in $\mu \mathrm{L}$ volumes per sample. Extraction techniques that are used to overcome these challenges are often expensive and time consuming however, this study presents a costeffective method for direct organic solvent to tissue extraction in combination with a d-SPE clean up that does not require large volumes of solvent compared to conventional SPE techniques.

The presented work is an expansion of a previously liver extraction focused on only fentanyl while this study expanded the panel to more fentanyl analogs. Overall, the method was validated following the 
criteria of the ASB 036 but has its limitations. The dilution integrity having a negative bias for all 34 analytes could be a weakness that can be improved even though it passed validation. A limitation of the study is not having paired matrix evaluation. Future work would be to test paired matrix samples (blood, liver, brain, etc) to draw conclusions and expand the value of the analysis to postmortem toxicology interpretations.

\subsubsection{Conclusions}

The QuEChERS extraction and subsequent LC-MS/MS method was fully validated using the ASB Standard 036 requirements for fentanyl, metabolites, and fentanyl analogs in liver tissue. The bias for all 34 target analytes at low, medium, and high concentrations did not exceed $\pm 20 \%$ with repeatability and reproducibility within the $20 \%$ threshold. The QuEChERS technique had recoveries $>93.3 \%$, MEs > 76.8\% and process efficiencies $>81.5 \%$ for all 34 target analytes. For storage in the autosampler, the samples are stable for 48 hours for all 34 fentanyl analogs and all but valeryl fentanyl were stable for 72 hours. Of the 22 authentic postmortem samples, 17 were multidrug positive with at least one fentanyl analog and fentanyl present indicating a prevalence of multiple fentanyl types in use. Nine of the 22 samples contained at least two fentanyl analogs plus fentanyl and metabolites. Three of the 22 samples contained three fentanyl analogs plus fentanyl and metabolites.

Overall, the QuEChERS extraction met all the acceptable criteria set by the ASB Standard 036 requirements and was shown to be effective at extracting and quantitating fentanyl and fentanyl analogs. The presented QuEChERS protocol meets all the requirements for liver extraction and proved to be effective at analyzing authentic liver specimens.

With the number of fentanyl overdose-related deaths continuing to increase, the interpretation of toxicological findings in these cases is crucial. The presented work consists of a comprehensive strategy to facilitate the extraction of novel synthetic opioids (NSO) including fentanyl and its major metabolites from a complex biological specimen, liver tissue. This chapter presented a sensitive and effective 
extraction strategy for liver tissue, an analytical method for 34 analytes to encompass potential emerging NSO's, and a survey of which specific NSO's are prevalent in the drug overdose community. This chapter provides an advancement in the combat of opioid epidemic by offering a fully validated protocol that complement the analytical toolkit in forensic toxicology laboratories and medical examiners offices. 
Chapter 4: Evaluation of the Effects of Fentanyl on Blowfly Larva and Application of QuEChERS Extraction Method for the Quantitation of Fentanyl and Metabolites from Larva and Pupa (Lucilia sericata) 


\subsection{Chapter Overview}

In this chapter, section 1 presents the validation of the extraction method for the analysis of fentanyl and metabolites from larva and pupa tissue for part of task 1.b. Also presented is the preliminary study for the purpose of validating the extraction method with extraction from authentic insect samples.

Section 2 of this chapter presents the morphometric comparisons for the larva and pupa reared in task 2.a. For all the insect stages, physical characteristics were recorded to evaluate the effects each treatment had on growth (task 2.b). To determine the effects of fentanyl on the growth and development of the blow flies, the mass, and percent stage (first instar, second instar, third instar, or pupa) of the insect were statistically compared using nested ANOVA (task 2.c). Tukey-HSD test will be applied to determine which treatments are significantly different within each replicate. These statistical tests will determine if the drug treatments have any significant effects on the insect's development. Age estimation using the mean length will be presented as well as the maximum length collected at each day.

Section 3 of this chapter presents the toxicological findings for the larva and pupa for task 1.e. The presented modified QuEChERS extraction was used for extraction of the feeding media liver and the insect tissue. The survey of fentanyl and metabolites detected in the larva and pupa is presented in this section. For task 3.a, a regression plot was created for a comparison of the fentanyl concentrations detected in the insect tissue to the liver fentanyl concentrations to establish any correlation of the concentrations.

\subsection{Section 1: Validation of QuEChERS Extraction for Larva and Pupa}

\subsubsection{Introduction}

Entomological techniques are utilized in forensic investigations to estimate the minimum time since death-this approach, consisting of using the growth and development of insects. Maggots follow a predictable life cycle that xenobiotics can influence. Even though entomology can be used to estimate the ${ }_{\min } \mathrm{PMI}$ due to the predictable nature of insect growth, the base assumption of predictable growth in a given environment is violated when insects are affected by xenobiotic influences if drugs or poisons 
present in the cadaver they have colonized (43-45). Forensic entomotoxicology, an area of growing interest, is concerned with the detection of drugs or toxins in insects, and exploring the implications such materials may pose on insect development $(42,50)$. For instance, recent reports including methamphetamine, cocaine, ketamine, morphine, and tramadol have shown contrasting effects on insect development (45-49). Stimulant drugs such as methamphetamine and cocaine, appeared to have an increasing effect on the physical development rate and body size of the blow flies $(46,48)$. It was found that the overall developmental time was shorter in the presence of cocaine, and the general insect growth being larger for both cocaine (Chrysomya albiceps (Wiedemann) and Chrysomya putoria (Wiedmann) (Diptera: Calliphoridae)) and methamphetamine laced diets (Calliphora stygia (Fabricius) (Diptera: Calliphoridae)) $(46,48)$. Among depressant-type drugs, morphine was shown to have no significant effect on size or development rates of $C$. stygia (47); however, ketamine and tramadol appeared to increase body size for the larvae of Lucilia sericata (Meigen) (Diptera: Calliphoridae) $(45,49)$. Changes in physical characters or developmental rates of insects due to the presence of xenobiotics can significantly impact ${ }_{\min } \mathrm{PMI}$ estimations, leading to either under- or overestimation of time since death (46). Understanding the effects that drugs can have on insect growth and development is important in terms of estimating $\min \mathrm{PMI}$ in cases that involve toxins or overdose deaths (50). Bioaccumulation of drugs and toxins in insect life stages has been documented and used to corroborate cause of death as well $(39,50,58,59,110)$. In instances with advanced decomposition, exsanguination, and even burn victims, traditional biological matrices of fluid or tissue may not be available or additional matrices of analysis for corroboration may be needed $(39,50,59)$.

In 2019, there were 49,860 reported deaths in the United States, due to overdose of opioids such as heroin and oxycodone and 36,359 deaths related to synthetic opioids including fentanyl (85). Fentanyl is of particular concern as it is a highly effective $\mu$-opioid agonist up to 50-100 times as potent as morphine (91). Mixed-drug preparations of heroin with fentanyl and/or other synthetic opioids also associated with 
a large number of deaths $(113,143)$. In response to epidemic, our research group is currently focused on the design and development of novel extraction approaches that facilitates the isolation, purification, enrichment of fentanyl and other novel psychoactive substances in different biological matrices, including indirect sources such as necrophilous insects.

An effective extraction of fentanyl from the insect matrix is a critical step to provide an accurate interpretation on drug presence and overall developmental outcomes. Heroin, methadone, morphine, and tramadol are exemplars that have been tested over insect matrices and subjected to extraction mechanisms with varying performance rates $(43-45,47,144)$. Previous studies have shown limitations to extract methamphetamine from larval stages but better success rates at detecting the drug in later stages of pupae, empty puparia, and adults (46). The inability to extract this stimulant drug from the larval stages was attributed to the limitations of the methanolic extraction used in combination with the lipid content of larval tissue $(46,145)$. Therefore, releasing the drug from the matrix plays a significant role in gaining insight on drug content. To this end, emerging techniques such as QuEChERS are necessary when dealing with tissue specimens. QuEChERS stands for quick, easy, cheap, effective, rugged, and safe and it has been reported as a versatile extraction technique to process human liver tissue, blood, and hair $(4,79,146-$ 148). This research work will expand the scope of application towards the extraction of larva and pupa tissue following specific validation guidelines from the Academy Standard Board 036. A quantitative method using tandem mass spectrometry for the quantitation of fentanyl and metabolites using tandem mass spectrometry is also reported.

While several drugs have been explored in entomotoxicological studies, little is known about the direct effects that fentanyl may pose on the growth and development of blow fly larvae. Therefore, it is crucial to understand the role of this drug in the development of Lucilia sericata blow flies. The overarching goals of this study are to evaluate the developmental effects of fentanyl on immature stages 
of the blow fly Lucilia sericata (Meigen)) and to monitor the prevalence of fentanyl and metabolites from larval and pupal tissue after feeding on liver tissue with the presence of fentanyl.

\subsubsection{Methods}

\subsubsection{Adult Colony}

Adult colonies of L. sericata were established in May 2018 from field collected specimens from Morgantown, West Virginia, USA. The collected insects were allowed to grow to adulthood and adults were identified as L. sericata by physical morphological characters (62). The colony was housed in BugDorm1 (MegaView Co., Ltd., Taiwan) screen cages $(30 \mathrm{~cm} \times 30 \mathrm{~cm} \times 30 \mathrm{~cm}$ ) stored in a Percival I36LLVLC8 incubator (Percival Scientific, Inc., Perry, lowa, USA) at $25^{\circ} \mathrm{C}\left( \pm 0.5^{\circ} \mathrm{C}\right)$ at $65 \%$ relative humidity ( $\pm 10 \%)$ with a 12:12 (L:D) photoperiod. Adults were given honey and water ad libitum and raw beef liver was provided for oviposition. New generations were separated from adults after oviposition to signal the beginning of the next generation and were moved to a new screen cage during emergence.

\subsubsection{Experimental Design}

In order to truly test the ability of the QuEChERS extraction to extract fentanyl and metabolites from larva and pupa tissue, authentic insects that feed on tissue fortified with fentanyl were required. To create authentic insects as toxicology specimens, fentanyl was spiked into $20 \mathrm{~g}$ aliquots of human liver homogenate to evaluate four concentrations: control $(0 \mu \mathrm{g} / \mathrm{kg})$, low $(10 \mu \mathrm{g} / \mathrm{kg})$, medium $(100 \mu \mathrm{g} / \mathrm{kg})$, and high $(350 \mu \mathrm{g} / \mathrm{kg})$ concentrations. To each aliquot of liver, approximately 50-60 eggs (by mass) were placed and allowed to feed undisturbed until time of collection. Egg mass estimates were generated by weighing 16 egg masses (average $n=26$ ) and by calculating the mass of one egg. The average weight of one egg was used to divide the mass of the eggs placed on the livers to estimate the number of eggs placed. For the larval treatment, third instar larvae at day 4 post transfer. For the pupal treatment, pupae were collected at day 12 after transfer. Larvae were collected and heat killed with boiling water for $90 \mathrm{~s}$ and rinsed with methanol before stored at $-20{ }^{\circ} \mathrm{C}$ with no liquid preservatives added $(7,40,46)$. Collected pupae were rinsed with deionized water and then methanol before being stored at $-20^{\circ} \mathrm{C}$ with no addition 
of any liquid preservative. No preservative was chosen due to the larval cuticle acting as a semipermeable membrane which can allow water or ethanol to diffuse into the body cavity and potential to leech out the target analytes into the preservative solution (149). To evaluate developmental effects, the mass (mg), length $(\mathrm{mm})$, and width $(\mathrm{mm})$ of the collected insects were documented (Figure 4.1). Metrics also evaluated were survivor rates, percent stage, and percent pupated. Statistical analysis was not performed as there was only one replicate for each collection day, the measurement of physical characters was purely exploratory. Insects from each treatment level and day of collection were randomly selected $(n=5)$ and extracted using the validated QuEChERS extraction. Larval specimens were also randomly selected into pools of four for triplicate analysis of pooled samples $(n=3)$.

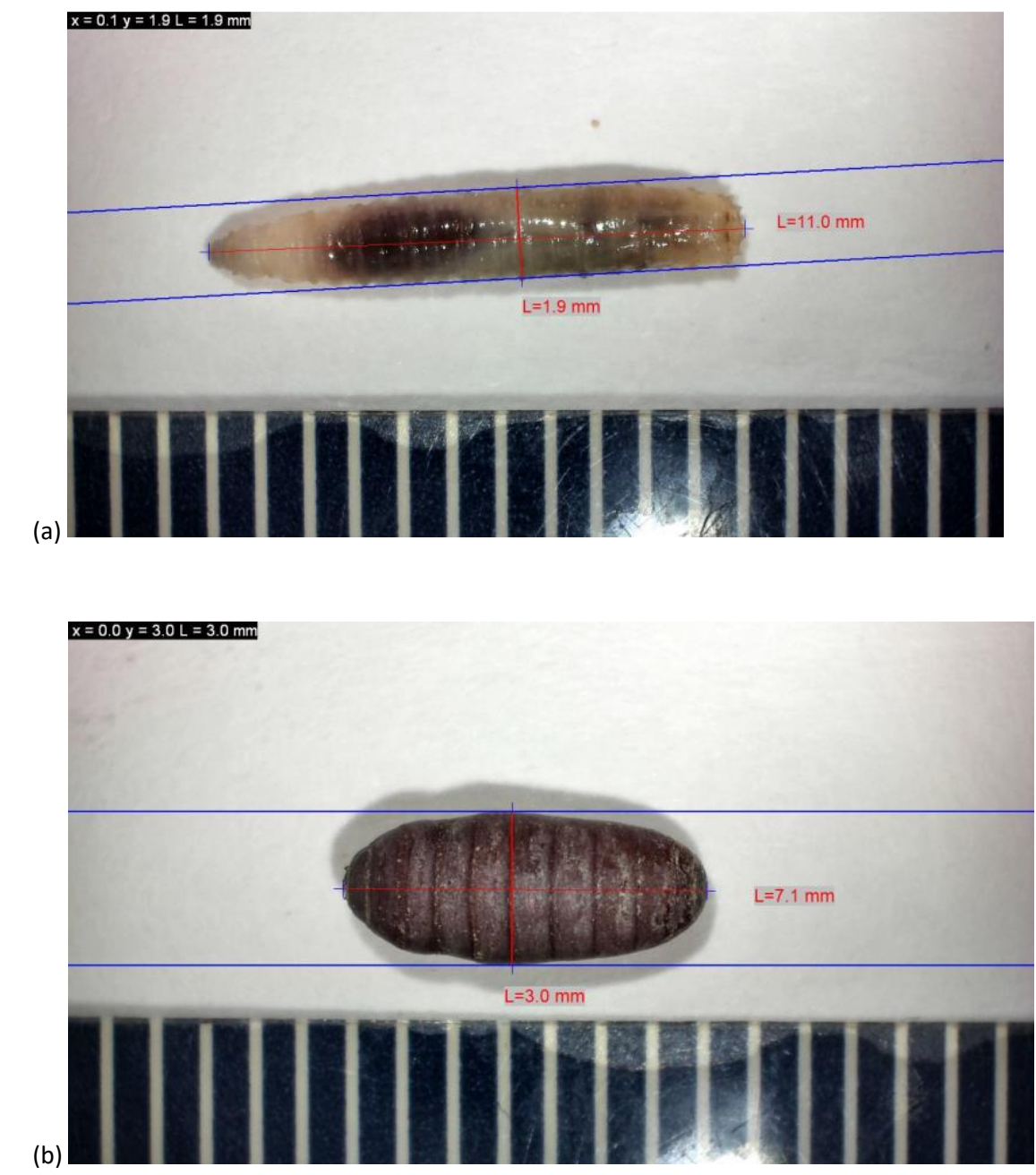

Figure 4.1: Larvae (day 4) and pupae (day 12) measurements of length and width (mm). 


\subsubsection{Chemicals and Materials}

Drug standards: fentanyl, norfentanyl, 4-ANPP, fentanyl- $D_{5}, 4-A N P P-D_{5}$, and norfenanyl- $D_{5}$ were obtained from Cerilliant (Round Rock, Tx) at concentrations of $1 \mathrm{mg} / \mathrm{mL}$ or $100 \mu \mathrm{g} / \mathrm{mL}$ in methanol. $\beta$ hydroxy fentanyl (hydrochloride) was purchased from Cayman Chemical (Ann Arbor, MI) and was reconstituted to a concentration of $100 \mu \mathrm{g} / \mathrm{mL}$ in methanol. Optima ${ }^{\circledR}$ liquid chromatography-mass spectrometry (LC-MS) grade methanol, acetonitrile, formic acid, and ammonium formate were purchased from Thermo Fisher Scientific (Fair Lawn, NJ). Water was purified using a Direct-Q 3 UV Water Purification System (Darmstadt, Germany). The QuEChERS original extraction salt packets (containing $6 \mathrm{~g}$ magnesium sulfate and $1.5 \mathrm{~g}$ sodium chloride) and $2 \mathrm{~mL}$ dispersive-SPE tubes (containing $25 \mathrm{mg}$ of primary secondary amine, $25 \mathrm{mg}$ end-capped octadecylsilane $\left(\mathrm{C}_{18} \mathrm{EC}\right)$ and $150 \mathrm{mg}$ magnesium sulfate) were purchased from Agilent Technologies. A Mixer Mill MM 200 was utilized for homogenization and was obtained from Retsch (Haan, Germany). Steel zinc plated BB balls $(0.177 \mathrm{cal}, 4.5 \mathrm{~mm})$ were obtained from Daisy (Rogers, AR) and were cleaned with hexane before use.

\subsubsection{LC-MS/MS}

Quantitation of fentanyl and metabolites was performed using an Agilent 6470 Triple Quadrupole system coupled with an Agilent 1290 Infinity II LC system. A Zorbax Eclipse Plus C $_{18}$ RRHD 3.0 x 100 mm, $1.8 \mu \mathrm{m}$ column was used for chromatographic separation using mobiles phases of $0.1 \%$ formic acid and 5 $\mathrm{mM}$ ammonium formate in water (mobile phase $\mathrm{A}$ ) and $0.1 \%$ formic acid in methanol (mobile phase B). Data acquisition was performed in dynamic multiple reaction monitoring (dMRM) mode with positive ESI with a principal dMRM transition for quantitation and one qualifier dMRM transition for each analyte. For chromatographic separation a gradient elution at a flow rate of $0.3 \mathrm{~mL} / \mathrm{min}$ was run as follows: initial hold for $0.5 \mathrm{~min}$ at $60 \%$ mobile phase B with a ramp to $65 \%$ for $3 \mathrm{~min}$, followed by a ramp to $95 \%$ for $0.5 \mathrm{~min}$ with a hold for $1 \mathrm{~min}$ and a $3 \mathrm{~min}$ post run for re-equilibrium at $60 \% \mathrm{~B}$. Chromatographic separation is shown in Figure 4.2. 


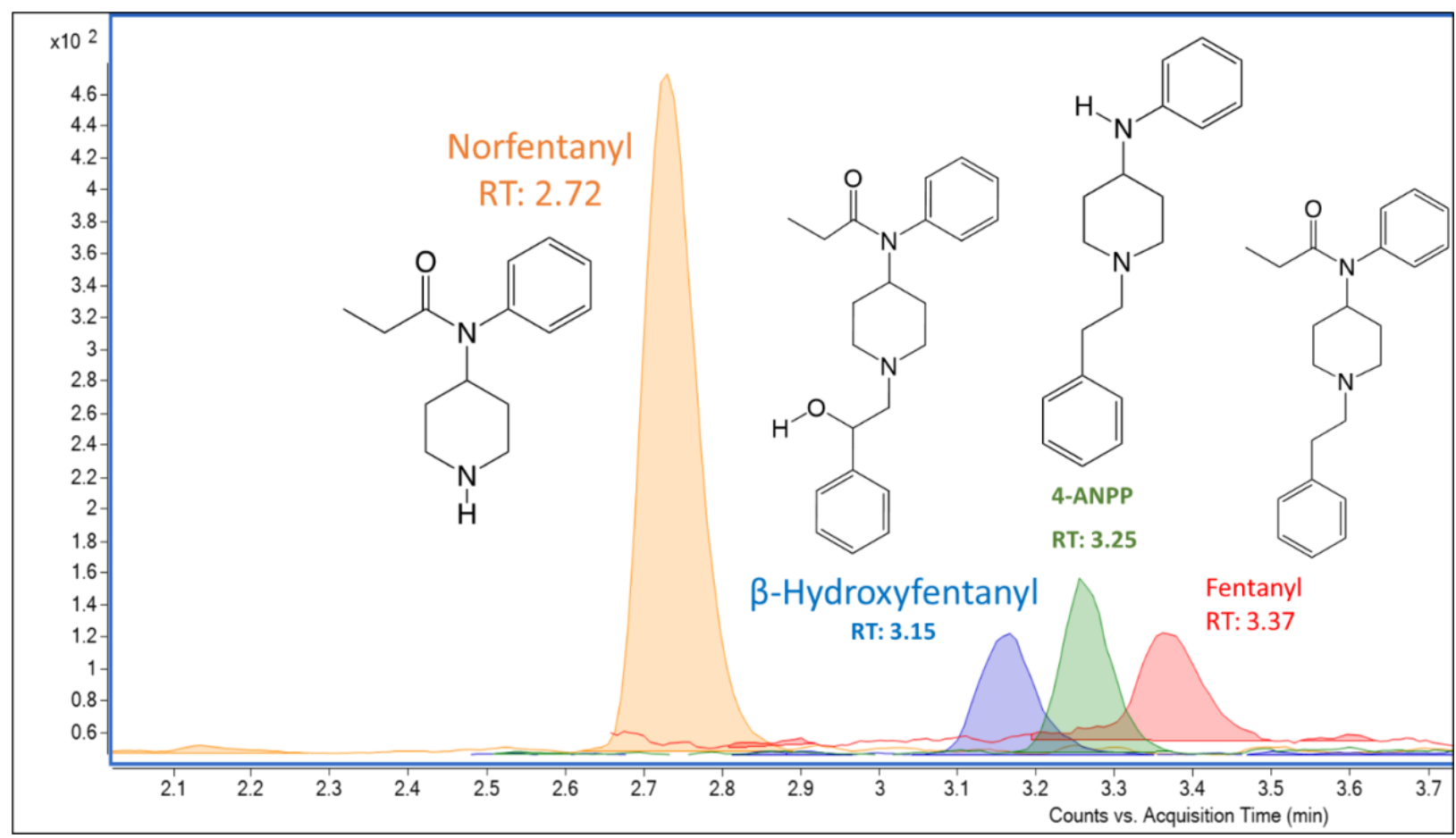

Figure 4.2: Chromatographic separation of norfentanyl, $\beta$-hydroxy fentanyl, 4-ANPP, and fentanyl at the LLOQ. Quantifier transitions are displayed above.

Agilent MassHunter Optimization software version B.08.02 was used to optimize the dMRM transitions for the optimal precursor ions with corresponding fragmentor voltage and selection of product ions with associated collision energies (CE). The selected parameters are listed in Table 4.1. MS source parameters: gas temperature $325^{\circ} \mathrm{C}$; gas flow $9 \mathrm{~L} / \mathrm{min}$; nebulizer 30 psi; sheath gas flow $10 \mathrm{~L} / \mathrm{min}$; capillary voltage $3500 \mathrm{~V}$ and charging voltage $500 \mathrm{~V}$. Quantitation was performed using Agilent MassHunter Quantitative software version B.08.00. Relative responses were calculated using the absolute response of each drug in relation to the response of the corresponding deuterated internal standard and plotted against expected drug concentrations for the calibrators. 
Table 4.1: MRM MS method parameters.

\begin{tabular}{|c|c|c|c|c|c|}
\hline Compound & Transition $^{\mathrm{a}}$ & $\begin{array}{l}\text { Fragmentor } \\
\text { (V) }\end{array}$ & $\mathrm{CE}(\mathrm{V})$ & Cell Acc (V) & Internal Standard \\
\hline \multirow{3}{*}{ Fentanyl } & $337.2 \rightarrow 188.0$ & \multirow{3}{*}{146} & 24 & \multirow{3}{*}{4} & \multirow{3}{*}{ D5-Fentanyl } \\
\hline & & & & & \\
\hline & $337.2 \rightarrow 105.0$ & & 48 & & \\
\hline \multirow{2}{*}{ 4-ANPP } & $281.2 \rightarrow 188.0$ & \multirow{2}{*}{122} & 16 & \multirow{2}{*}{4} & \multirow[t]{2}{*}{ D5-4-ANPP } \\
\hline & $281.2 \rightarrow 105.0$ & & 36 & & \\
\hline \multirow{2}{*}{ Norfentanyl } & $233.2 \rightarrow 84.0$ & \multirow{2}{*}{117} & 20 & \multirow{2}{*}{4} & \multirow{2}{*}{ D5-Norfentanyl } \\
\hline & $233.2 \rightarrow 150.0$ & & 20 & & \\
\hline \multirow[b]{2}{*}{$\beta$-Hydroxyfentanyl } & $353.2 \rightarrow 91.0$ & \multirow[b]{2}{*}{144} & 56 & \multirow[b]{2}{*}{4} & \multirow[b]{2}{*}{ D5-Fentanyl } \\
\hline & $353.2 \rightarrow 132.1$ & & & & \\
\hline \multirow[b]{2}{*}{ D5-Fentanyl } & $342.3 \rightarrow 188.0$ & \multirow{2}{*}{141} & 24 & \multirow[b]{2}{*}{4} & \multirow[b]{2}{*}{-} \\
\hline & $342.3 \rightarrow 105.0$ & & 48 & & \\
\hline \multirow{2}{*}{ D5-Norfentanyl } & $238.2 \rightarrow 84.0$ & \multirow{2}{*}{107} & 20 & \multirow[b]{2}{*}{4} & \multirow[b]{2}{*}{-} \\
\hline & $238.2 \rightarrow 55.1$ & & 48 & & \\
\hline \multirow{2}{*}{ D5-4-ANPP } & $286.2 \rightarrow 188.1$ & \multirow{2}{*}{114} & 20 & \multirow{2}{*}{4} & \multirow{2}{*}{ - } \\
\hline & $286.2 \rightarrow 105.0$ & & 40 & & \\
\hline
\end{tabular}

${ }^{a}$ Precursor ion followed by product ion, quantifier transition is bold

\subsubsection{Sample preparation}

The QuEChERS extraction was validated using a target sample aliquot of $0.2 \mathrm{~g}$ of insect tissue larval or pupal as appropriate. To verify spiking concentration, liver specimens were extracted at $0.2 \mathrm{~g}$ aliquots and were quantified using a previously validated protocol (4). Larva specimens were weighed for extraction as single samples and pools of four insects for triplicate analysis $(n=3)$ with the results presented as $\mu \mathrm{g} / \mathrm{kg}$. Pupa specimens were analyzed only as individual samples due to low survival rates and low number of insects collected. Insect specimens were placed into a $1.5 \mathrm{~mL}$ centrifuge tube, followed by $400 \mu \mathrm{L}$ deionized water, $100 \mu \mathrm{L}$ internal standard mix (IS), and $700 \mu \mathrm{L}$ acetonitrile combined with three steel beads. Afterwards, $0.2 \mathrm{~g}$ of QuEChERS original extraction salt was added to the tubes, vortexed, mixed on a mixer mill for $3 \mathrm{~min}$, centrifuged for $5 \mathrm{~min}$, and the resulting supernatant was transferred to a QuEChERS dispersive-SPE tube. Tubes were vortexed then centrifuged for $5 \mathrm{~min}$ before the supernatant 
was transferred to a new $1.5 \mathrm{~mL}$ microcentrifuge tube for dry down under a nitrogen stream at $50{ }^{\circ} \mathrm{C}$ to dryness. Samples were reconstituted with $100 \mu \mathrm{L}$ methanol and transferred to vials for LC-MS/MS analysis. This extraction protocol was adapted and validated for larva and pupa tissue from work published by Cox et al. for the extraction of liver tissue (4). The IS was comprised of three deuterated compounds: fentanyl- $D_{5}$, norfentanyl- $D_{5}$, and 4-ANPP- $D_{5}$ at a concentration of $1 \mathrm{ng} / \mathrm{mL}$.

\subsubsection{Validation parameters}

The QuEChERS extraction and LC-MS/MS analysis was validated following the American Standards Board (ASB) 036 requirements (104). Evaluated parameters included calibration model, interference studies, ionization suppression/enhancement, dilution integrity, limits of quantitation, processed sample stability, bias, and precision.

\subsection{Calibration model}

A calibration stock mix of fentanyl ( $2 \mathrm{ng} / \mu \mathrm{L})$, 4-ANPP ( $2 \mathrm{ng} / \mu \mathrm{L})$, norfentanyl (10 $\mathrm{ng} / \mu \mathrm{L})$, and $\beta$ hydroxy fentanyl $(8 \mathrm{ng} / \mu \mathrm{L})$ was created for spiking tissues in the calibration curve and represents the highest calibrator. Using this solution, a serial dilution was performed to create stock solutions at concentrations of $0.004,0.008,0.04,0.2,1$, and $2 \mathrm{ng} / \mu \mathrm{L}(\mathrm{ppm})$ for fentanyl and 4-ANPP with concentrations of $0.02,0.04,0.2,1,5$, and $10 \mathrm{ng} / \mu \mathrm{L}(\mathrm{ppm})$ for norfentanyl and $0.012,0.032,0.16,0.8,4$, and $8 \mathrm{ng} / \mu \mathrm{L}(\mathrm{ppm})$ for $\beta$-hydroxy fentanyl. To each $0.2 \mathrm{~g}$ insect calibrator sample, $5 \mu \mathrm{L}$ of each stock solution was added to create the calibration curve.

\subsection{Interference studies}

To evaluate interferences a high concentration (highest calibrator) of the target analytes was injected with no IS to show any interference of the drug standards to the IS. Conversely, an aliquot of IS with no target analytes was injected to observe any interference caused by the IS. Matrix interferences were evaluated by extracting blank matrix sources $(n=10)$ for both larva and pupa with no IS added. To evaluate potential interferences from commonly encountered compounds, a mix of common drugs encountered in toxicology ( $n=31)$ was injected (Table 4.2). 
Table 4.2: List of 31 compounds evaluated for potential interferences.

\begin{tabular}{|c|c|c|c|}
\hline Class & Compound & Class & Compound \\
\hline \multirow[t]{6}{*}{ Opioids } & 6-Acetylmorphine & Synthetic Cannabinoids & JWH-018 \\
\hline & Oxycodone & & JWH-073 \\
\hline & Hydrocodone & & XLR-11 \\
\hline & Buprenorphine & & AB-FUBINACA \\
\hline & Norbuprenorphine & & AB-PINACA \\
\hline & Ethylmorphine & & MAM2201 \\
\hline \multirow[t]{2}{*}{ Opiates } & Morphine & Stimulants & Amphetamine \\
\hline & Codeine & & Methamphetamine \\
\hline \multirow[t]{9}{*}{ Supplements } & 1S,2R (+)-Ephedrine & & Cocaine \\
\hline & Methylphenidate & Synthetic Cathinones & $\begin{array}{c}\text { Alpha- } \\
\text { Pyrrolidinopentiophenone }(\alpha \\
\text {-PVP) }\end{array}$ \\
\hline & Sibutramine & & $\begin{array}{l}\text { 3,4-Methylenedioxy } \\
\text { Pyrovalerone }\end{array}$ \\
\hline & & & (MDPV) \\
\hline & Caffeine & Cannabinoids & Delta-9 THC (THC) \\
\hline & Synephrine & & Cannabidiol (CBD) \\
\hline & Octopamine & Other Alkaloids & Mitragynine \\
\hline & Methylhexanamine & & \\
\hline & (DMAA) & & \\
\hline
\end{tabular}

\subsection{Ionization suppression/enhancement}

lonization suppression or enhancement was evaluated at two concentrations, low and high, for ten replicates at both concentrations following the ASB Standard 036 requirements. To be deemed acceptable, the percent ionization suppression or enhancement shall not exceed $\pm 25 \%$ with a percent relative standard deviation (\%CV) not exceeding $20 \%$ (104). A post-extraction addition approach was utilized by comparing neat standards (Set 1 ) to blank matrix samples fortified with neat standard after extraction (Set 2). Set 1 consisted of neat standards prepared at low ( 2 times the LLOQ) and high (50\% of the highest calibrator) and were injected ten times to establish the mean peak area for each target analyte. Set 2 consisted of ten individual blank matrix sources (larva and pupa) in duplicate, for low ( $\mathrm{n}=10$ ) 
and high $(n=10)$ concentration, extracted and spiked with the low and high concentration, respectively, after extraction. The average area of each set $(\bar{X})$ was used to calculate the suppression or enhancement effects at each concentration for each analyte is as follows:

$$
\text { Ionization suppression or enhancement }(\%)=\left(\frac{\bar{X} \text { Area of set } 2}{\bar{X} \text { Area of set } 1}-1\right) * 100
$$

\subsection{Bias and Precision}

For bias and precision calculations, blank larval and pupal tissue samples were spiked and analyzed at low, medium, and high concentrations for fentanyl and metabolites in triplicate over five days $(n=15)$. Bias was calculated as a percent deviation of the extracted mean concentration from the theoretical concentration with an acceptable bias not exceeding $\pm 20 \%$. Precision was evaluated by percent standard deviation $(\% \mathrm{CV})$ of the mean at each concentration with the criteria of not exceeding $20 \%$.

\subsubsection{Limits}

For the evaluation of the limit of detection (LOD) and the lower limit of quantitation (LLOQ), blank matrix sources for larval and pupal tissue were spiked with concentrations at the lowest non-zero calibrator. Triplicates of each blank matrix sources were spiked at the lowest non-zero calibrator and analyzed over three days $(n=9)$. The acceptable parameters were bias $( \pm 20 \%)$ and precision $(\% C V \leq 20 \%)$. For LOD, chromatographic acceptance was evaluated and deemed acceptable when signal to noise $(\mathrm{s} / \mathrm{n}$ ) ratios were above 3.3 and qualifier ratios did not exceed $\pm 20 \%$.

\subsubsection{Carryover and Dilution Integrity}

Blank matrix samples were injected directly after injections of samples three times greater than the highest calibrator in triplicate analysis to evaluate carryover. Carryover was not considered significant if present below $10 \%$ of the lowest calibrator (LLOQ). For dilution integrity, blank matrix samples from both sources were spiked at concentrations three times the highest calibrator in triplicate and extracted 
simultaneously with blank matrix only spiked with internal standard in triplicate. The resulting extract was diluted 1:5 (S:T) with the extracted blank matrix with internal standard $(5,106)$. The process was repeated over a period of five runs $(n=15)$ and the dilution integrity was deemed acceptable if bias did not exceed $\pm 20 \%$ and the precision (\%CV) did not exceed $20 \%$.

\subsubsection{Processed Sample Stability}

To determine the stability of processed samples for both larval and pupal tissue in the autosampler $\left(25^{\circ} \mathrm{C}\right)$, blank larva and pupa samples were spiked at low and high concentrations and extracted. Post extraction reconstituted samples, for low and high respectively, were pooled and aliquoted to LC-MS/MS vials. Triplicate analysis at time frames: $0,12,24,36,48,60$, and $72 \mathrm{hr}$ were performed for each concentration. Average peak area was calculated for each triplicate and compared to time zero. An acceptable bias threshold was set at $\pm 20 \%$.

\subsubsection{Recovery and Process Efficiency}

To calculate recovery of the extraction (RE) and process efficiency (PE) two formulas were utilized. Variables in the formulas represent the mean peak areas for the neat solution (A), the mean peak areas for the standards spiked after extraction (B), and the mean peak areas for the standards spiked before extraction (C) (107). Recovery and process efficiency were at low and high concentrations for ten replicates $(n=10)$ for each matrix type. Ideally, a perfect recovery or process efficiency would be $100 \%$ and $\pm 20 \%$ of this value is desired while not exceeding a $\%$ CV of $20 \%$. The ASB Standard 036 does not have a requirement for recovery or process efficiency and the $\pm 20 \%$ while not exceeding a $\% C V$ of $20 \%$ is a selfimposed guideline. 


$$
\begin{aligned}
& R E(\%)=\frac{C}{B} * 100 \\
& P E(\%)=\frac{C}{A} * 100
\end{aligned}
$$

\subsubsection{Results}

\subsubsection{Validation}

Six different concentrations for five runs $(n=15)$ were analyzed for both larva and pupa tissue by linear regression lines generated using Agilent MassHunter software with a weight of $(1 / x)$. The linear range or analytical measurement range (AMR) is displayed in Table 4.3 for each analyte in both matrices. As part of the evaluation of the calibration model, the $R^{2}$ values for the calibration curves exceeded 0.99 and the residuals for each set of five replicates were plotted with no discernable pattern being identified indicating a random dispersion. This allows for linear analysis of the analytes presented using a weight of $(1 / x)$

\begin{tabular}{|c|c|c|c|c|}
\hline \multicolumn{5}{|c|}{ Larvae } \\
\hline \multirow{2}{*}{ Compound } & \multirow{2}{*}{$\begin{array}{c}\text { LOD/LLOQ } \\
\quad(\mu \mathrm{g} / \mathrm{kg})\end{array}$} & \multicolumn{2}{|c|}{ LLOQ (n=9) } & \multirow{2}{*}{$\begin{array}{c}\text { AMR } \\
(\mu \mathrm{g} / \mathrm{kg})\end{array}$} \\
\hline & & Bias & $\% \mathrm{CV}$ & \\
\hline 4-ANPP & 0.1 & -3.4 & 12.1 & $0.1-50$ \\
\hline$\beta$-Hydroxyfentanyl & 0.4 & 1.7 & 9.0 & $0.4-200$ \\
\hline Fentanyl & 0.1 & -1.5 & 14.6 & $0.1-50$ \\
\hline Norfentanyl & 0.5 & 4.6 & 12.1 & $0.5-250$ \\
\hline \multicolumn{5}{|c|}{ Pupae } \\
\hline \multirow{2}{*}{ Compound } & \multirow{2}{*}{$\begin{array}{c}\text { LOD/LLOQ } \\
(\mu \mathrm{g} / \mathrm{kg})\end{array}$} & \multicolumn{2}{|c|}{ LLOQ (n=9) } & \multirow{2}{*}{$\begin{array}{c}\text { AMR } \\
(\mu \mathrm{g} / \mathrm{kg})\end{array}$} \\
\hline & & Bias & $\% \mathrm{CV}$ & \\
\hline 4-ANPP & 0.1 & -6.0 & 13.2 & $0.1-50$ \\
\hline$\beta$-Hydroxyfentanyl & 0.4 & -4.6 & 12.7 & $0.4-200$ \\
\hline Fentanyl & 0.1 & -11.1 & 10.5 & $0.1-50$ \\
\hline Norfentanyl & 0.5 & 3.9 & 16.8 & $0.5-250$ \\
\hline
\end{tabular}

Table 4.3: LOD, LLOQ, and AMR for larvae and pupae extractions. 
Blank matrix samples from 10 individual insect sources for both larval and pupal tissue were extracted without addition of standards or internal standards to determine any matrix interferences. Chromatographic analysis of these injections was performed using Agilent MassHunter Qualitative Analysis software version B.08.00 and no interference peaks from the blank matrices were observed.

No interfering peaks were observed from the high standards to the internal standards or from the internal standards to the target analytes. A neat mix of 31 commonly encountered analytes, encompassing other opioids, stimulants, depressants, synthetic cannabinoids, synthetic cathinones, and dietary supplements, was injected and no interferences were observed.

\subsubsection{Ionization suppression/enhancement}

Ionization suppression or enhancement was evaluated at low and high concentrations $(n=10)$ for both larval and pupal tissue following the ASB Standard 036 requirements. The percent ionization suppression or enhancement and \%CV for all four target analytes are presented in Table 4.4. The criteria for acceptance was $\pm 25 \%$ ionization suppression or enhancement with a percent relative standard deviation (\%CV) not exceeding $20 \%$. The four target analytes were within the criteria for acceptance at both high and low concentrations for both insect tissue types. For the larval tissue, the percent ionization suppression or enhancement for the four analytes ranged from $-10.9 \%$ to $17.9 \%$ for the low and ranged from $2.8 \%$ to $8.2 \%$ for the high while not exceeding a $\% \mathrm{CV}$ of $14.2 \%$. For the pupal tissue, the percent ionization suppression or enhancement ranged from $-5.5 \%$ to $1.1 \%$ at the low concentration and ranged from $3.0 \%$ to $8.8 \%$ for the high while not exceeding a $\%$ CV of $16.6 \%$. 
Table 4.4: Ionization suppression/enhancement, recovery, and process efficiency for the larval and pupal tissue ( $n=10)$.

\begin{tabular}{|c|c|c|c|c|c|c|c|c|c|c|c|c|}
\hline \multirow{3}{*}{ Compound } & \multicolumn{4}{|c|}{$\begin{array}{c}\text { Ionization } \\
\text { Suppression/Enhancement }^{\mathrm{a}} \\
\end{array}$} & \multicolumn{4}{|c|}{ Recovery ${ }^{\mathrm{a}}$} & \multicolumn{4}{|c|}{ Process Efficiency ${ }^{a}$} \\
\hline & \multicolumn{2}{|c|}{ Low } & \multicolumn{2}{|c|}{ High } & \multicolumn{2}{|c|}{ Low } & \multicolumn{2}{|c|}{ High } & \multicolumn{2}{|c|}{ Low } & \multicolumn{2}{|c|}{ High } \\
\hline & Mean & $\mathrm{CV}$ & Mean & $\mathrm{CV}$ & Mean & $\mathrm{CV}$ & Mean & $\mathrm{CV}$ & Mean & $\mathrm{CV}$ & Mean & $\mathrm{CV}$ \\
\hline \multicolumn{13}{|c|}{ Larvae } \\
\hline 4-ANPP & -3.5 & 4.8 & 6.6 & 8.7 & 96.3 & 13.8 & 83.7 & 11.4 & 92.9 & 13.2 & 89.2 & 13.0 \\
\hline $\begin{array}{c}\beta- \\
\text { Hydroxyfentanyl }\end{array}$ & 10.2 & 7.2 & 4.7 & 8.2 & 103.5 & 10.4 & 98.2 & 12.7 & 114.1 & 8.9 & 102.8 & 12.5 \\
\hline Fentanyl & -10.9 & 14.2 & 2.8 & 8.5 & 111.8 & 11.3 & 96.4 & 12.2 & 99.6 & 15.3 & 99.1 & 11.3 \\
\hline Norfentanyl & 17.9 & 6.9 & 8.2 & 9.2 & 97.4 & 12.0 & 101.0 & 12.7 & 114.9 & 10.8 & 109.2 & 11.9 \\
\hline \multicolumn{13}{|c|}{ Pupae } \\
\hline 4-ANPP & -5.5 & 9.3 & 3.0 & 5.4 & 103.9 & 6.2 & 104.3 & 12.3 & 98.2 & 8.5 & 107.4 & 12.1 \\
\hline $\begin{array}{c}\beta- \\
\text { Hydroxyfentanyl }\end{array}$ & -4.9 & 16.6 & 8.8 & 5.1 & 107.9 & 15.8 & 98.9 & 6.5 & 102.6 & 8.1 & 107.6 & 4.7 \\
\hline Fentanyl & -5.1 & 9.8 & 7.1 & 5.0 & 102.1 & 7.6 & 102.4 & 8.9 & 96.9 & 9.9 & 109.6 & 7.8 \\
\hline Norfentanyl & 1.1 & 12.0 & 6.4 & 7.3 & 94.5 & 7.1 & 104.3 & 9.0 & 95.6 & 12.6 & 111.1 & 6.5 \\
\hline
\end{tabular}

Data in \%

\subsubsection{Bias and precision}

For the bias and precision calculations, blank insect matrix was spiked and extracted at low, medium, and high concentrations in triplicate over five runs $(n=15)$ for both larva and pupa tissue (Table 4.5). The bias for all four analytes in both insect matrices were within $\pm 20 \%$ and the $\% C V$ did not exceed $20 \%$, meeting the ASB Standard 036 requirements. For the larval tissue, the bias for the low ranged from $-4.6 \%$ to $10.1 \%$, the medium ranged from $-3.5 \%$ to $5.0 \%$, and the high ranged from $-4.9 \%$ to $-2.0 \%$. For the pupal tissue, the bias for the low ranged from $-1.5 \%$ to $11.3 \%$, for the medium ranged from $-4.8 \%$ to $0.7 \%$, and for the high ranged from $-4.8 \%$ to $-0.4 \%$. Of the four analytes and two matrices, only $\beta$-hydroxy fentanyl experienced a bias greater than $\pm 10 \%$ at $10.1 \%$ bias for the low larva matrix and $11.3 \%$ bias for the low pupa matrix. These elevated percent bias values could be due to not having a deuterated internal standard for this analyte; however, the values are still well within the criteria for acceptance. 
Table 4.5: The bias and precision validation data for larvae and pupae extractions ( $n=15)$.

\begin{tabular}{|c|c|c|c|c|c|c|c|c|c|}
\hline \multicolumn{10}{|c|}{ Larvae } \\
\hline \multirow{2}{*}{ Compound } & \multicolumn{3}{|c|}{$\operatorname{Bias}(\%)$} & \multicolumn{3}{|c|}{ Between Run (\%CV) } & \multicolumn{3}{|c|}{ Within Run (\%CV) } \\
\hline & LOW & MED & HIGH & LOW & MED & HIGH & LOW & MED & $\mathrm{HIGH}$ \\
\hline 4-ANPPa & -4.6 & -3.5 & -3.8 & 10.6 & 8.5 & 8.7 & 10.8 & 6.8 & 7.0 \\
\hline$\beta$-Hydroxyfentanyl ${ }^{\mathrm{b}}$ & 10.1 & 5.0 & -2.0 & 9.5 & 7.5 & 5.4 & 9.5 & 5.3 & 3.7 \\
\hline Fentanyl $^{\mathrm{a}}$ & -2.9 & -1.4 & -2.4 & 8.5 & 7.9 & 5.7 & 8.1 & 4.3 & 3.6 \\
\hline \multirow[t]{2}{*}{ Norfentanyl $^{\mathrm{c}}$} & 0.1 & 1.5 & -4.9 & 12.2 & 7.2 & 7.0 & 7.0 & 3.8 & 4.1 \\
\hline & \multicolumn{6}{|c|}{ Pupae } & & & \\
\hline \multirow{2}{*}{ Compound } & \multicolumn{3}{|c|}{$\operatorname{Bias}(\%)$} & \multicolumn{3}{|c|}{ Between Run (\%CV) } & \multicolumn{3}{|c|}{ Within Run (\%CV) } \\
\hline & LOW & MED & HIGH & LOW & MED & HIGH & LOW & MED & $\mathrm{HIGH}$ \\
\hline 4-ANPPa & 0.1 & -3.8 & -4.8 & 8.9 & 4.7 & 7.0 & 8.8 & 3.7 & 8.4 \\
\hline$\beta$-Hydroxyfentanyl ${ }^{\mathrm{b}}$ & 11.3 & -0.7 & -0.4 & 8.4 & 5.1 & 6.8 & 6.7 & 4.4 & 6.2 \\
\hline Fentanyl $^{\mathrm{a}}$ & -1.5 & -1.3 & -2.2 & 12.1 & 8.0 & 5.1 & 6.8 & 5.5 & 5.9 \\
\hline Norfentanyl $\mathrm{l}^{\mathrm{c}}$ & 7.1 & -4.8 & -1.8 & 5.9 & 3.2 & 7.2 & 4.3 & 2.9 & 8.3 \\
\hline
\end{tabular}

\subsubsection{Limits}

Triplicates of individual larva and pupa matrix sources were spiked at the lowest non-zero calibrator and analyzed over three runs $(n=9)$ to satisfy the requirements for the limit of detection and lower limit of quantitation (104). Bias and precision for the LLOQ (Table 4.3) were considered acceptable within $\pm 20 \%$ for bias and the $\%$ CV not exceeding $20 \%$. The bias for the LLOQ for the larval tissue ranged from $-3.4 \%$ to $4.6 \%$ and for the pupal tissue ranged from $-11.1 \%$ to $3.9 \%$. The requirements for the LOD and LLOQ by the ASB Standard 036 were met for both matrices.

\subsubsection{Carryover and dilution integrity}

To determine any carryover, extracted blank matrix samples were injected directly after injections of samples with concentrations three times greater than the highest calibrator in triplicate analysis. This was performed for both the larval and pupal matrices. No significant carryover of the target analytes was detected in the blank matrix injections for either matrix.

For the dilution integrity evaluation, bias and precision was calculated for each analyte and is shown in Table 4.6. For the four target analytes in the larval tissue the dilution integrity did not exceed 
$\pm 12.7 \%$ for bias and did not exceed $14.1 \%$ for $\%$ CV. For the pupal tissue the dilution integrity did not exceed $\pm 9.7 \%$ for bias and did not exceed $11.2 \%$ for $\% C V$. For the larva extractions, the bias of the dilution integrity ranged from $-12.7 \%$ to $-5.9 \%$ indicating a tendency for the dilution to have a lower than anticipated value. In contrast, the dilution integrity bias for the pupa extracts ranged from $1.3 \%$ to $9.7 \%$ indicating a slightly inflated value than expected. This could be attributed to matrix effect at the high concentration for the pupa extraction as the pupa extractions at the high-end experience slight ionization enhancement.

Table 4.6: Dilution integrity for the larvae and pupae extractions at a 1:5 dilution.

\begin{tabular}{|c|c|c|c|}
\hline \multicolumn{4}{|c|}{ Larvae } \\
\hline Compound & Target Concentration $(\mu \mathrm{g} / \mathrm{kg})$ & Dilution Integrity (\% Bias) & $\% \mathrm{CV}$ \\
\hline 4-ANPP & 150 & -12.7 & 14.1 \\
\hline$\beta$-Hydroxyfentanyl & 600 & -5.9 & 10.7 \\
\hline Fentanyl & 150 & -10.3 & 13.5 \\
\hline Norfentanyl & 750 & -11.3 & 12.9 \\
\hline \multicolumn{4}{|c|}{ Pupae } \\
\hline Compound & Target Concentration $(\mu \mathrm{g} / \mathrm{kg})$ & Dilution Integrity (\% Bias) & $\% \mathrm{CV}$ \\
\hline 4-ANPP & 150 & 1.3 & 9.9 \\
\hline$\beta$-Hydroxyfentanyl & 600 & 9.7 & 11.2 \\
\hline Fentanyl & 150 & 7.8 & 10.1 \\
\hline Norfentanyl & 750 & 7.4 & 8.7 \\
\hline
\end{tabular}

\subsubsection{Processed sample stability}

Processed sample stability in the autosampler was evaluated for both insect matrices in triplicate at low and high concentrations over time intervals up to 72 hours (Table 4.7). For the larva extractions, all four analytes were stable up to $72 \mathrm{hr}$ at both the low and high concentrations. Norfentanyl changed the most in response with the low concentration increasing to $15.8 \%$ of the time zero response. For the pupa extractions, all the analytes were stable up to $72 \mathrm{hr}$ for both the low and high concentrations. The analyte with the most instability, 4-ANPP decreased in regard to the time zero response at both the low and high concentrations at $72 \mathrm{~h}$ with $-15.4 \%$ and $-14.3 \%$ respectively. 


\subsubsection{Recovery and process efficiency}

Recovery and process efficiency was evaluated simultaneously with ionization suppression or enhancement and the results are displayed in Table 4.4. The recoveries and process efficiencies for both matrices at the low and high concentrations were deemed acceptable within $\pm 20 \%$ of the target recoveries and the \%CV not exceeding $20 \%$. Overall, 4-ANPP and fentanyl recovered more efficiently in the pupa extractions compared to the larva extraction at both concentrations. Conversely, $\beta$-hydroxy fentanyl and norfentanyl had slightly better recoveries for the larva extractions compared to the pupa extractions. The precision for the recovery and process efficiency did not exceed $15.8 \%$ with the majority being below $13 \%$.

Table 4.7: Stability at a low and high concentration for the larvae and pupae extractions for processed samples stored in the autosampler $\left(25^{\circ} \mathrm{C}\right)$.

\section{Larvae}

\begin{tabular}{|c|c|c|c|c|c|c|c|c|c|c|c|c|}
\hline \multirow{2}{*}{ Compound } & \multicolumn{6}{|c|}{ Low $\left(\% \Delta^{\mathrm{d}}\right)$} & \multicolumn{6}{|c|}{$\operatorname{High}\left(\% \Delta^{\mathrm{d}}\right)$} \\
\hline & $12 \mathrm{~h}$ & $24 \mathrm{~h}$ & $36 \mathrm{~h}$ & $48 \mathrm{~h}$ & $60 \mathrm{~h}$ & $72 \mathrm{~h}$ & $12 \mathrm{~h}$ & $24 \mathrm{~h}$ & $36 \mathrm{~h}$ & $48 \mathrm{~h}$ & $60 \mathrm{~h}$ & $72 \mathrm{~h}$ \\
\hline 4-ANPPa & 3.8 & 0.7 & 1.5 & 1.5 & 1.8 & -4.8 & -1.8 & -3.2 & -3.4 & -1.0 & -2.8 & -12.7 \\
\hline$\beta$-Hydroxyfentanyl ${ }^{\mathrm{b}}$ & 2.9 & 0.1 & -3.1 & 5.8 & 7.1 & 2.9 & -0.1 & 1.4 & 0.0 & 4.9 & 4.6 & -3.1 \\
\hline Fentanyl $^{\mathrm{a}}$ & 3.4 & 4.5 & -4.4 & 3.2 & 7.5 & 0.0 & -0.9 & 1.3 & -0.5 & 1.8 & 1.2 & -6.6 \\
\hline Norfentanyl $^{\mathrm{c}}$ & 9.8 & 9.3 & 9.2 & 13.6 & 14.3 & 15.8 & -0.4 & -1.9 & -1.9 & 2.4 & 1.0 & -7.7 \\
\hline \multicolumn{13}{|c|}{ Pupae } \\
\hline \multirow[t]{2}{*}{ Compound } & \multicolumn{6}{|c|}{ Low $\left(\% \Delta^{\mathrm{d}}\right)$} & \multicolumn{6}{|c|}{$\operatorname{High}\left(\% \Delta^{\mathrm{d}}\right)$} \\
\hline & $12 \mathrm{~h}$ & $24 \mathrm{~h}$ & $36 \mathrm{~h}$ & $48 \mathrm{~h}$ & $60 \mathrm{~h}$ & $72 \mathrm{~h}$ & $12 \mathrm{~h}$ & $24 \mathrm{~h}$ & $36 \mathrm{~h}$ & $48 \mathrm{~h}$ & $60 \mathrm{~h}$ & $72 \mathrm{~h}$ \\
\hline 4-ANPPa & -2.6 & -10.8 & 1.1 & -5.7 & -7.5 & -15.4 & -6.2 & -11.0 & -1.1 & -8.8 & -6.8 & -14.3 \\
\hline$\beta$-Hydroxyfentanyl ${ }^{\mathrm{b}}$ & 2.8 & -3.7 & 3.0 & -1.9 & 0.2 & 2.2 & -4.6 & -6.9 & 2.7 & -2.2 & 0.7 & -4.2 \\
\hline Fentanyl $^{\mathrm{a}}$ & -3.4 & -5.0 & 2.9 & -4.4 & -2.9 & -1.6 & -4.6 & -6.6 & 1.0 & -4.7 & -2.3 & -7.6 \\
\hline Norfentanyl $^{\mathrm{c}}$ & 3.9 & -3.1 & 6.2 & 9.2 & 14.0 & 5.1 & -3.6 & -9.2 & -2.2 & -2.9 & -0.7 & -8.9 \\
\hline $\begin{array}{l}\text { a Low concentration at } 0.2 \\
\text { b Low concentration at } 0.8 \\
\text { c Low concentration at } 1 \mu\end{array}$ & $\mathrm{kg}$, and & igh conc & tration & $25 \mu \mathrm{g} / \mathrm{k}$ & & & & & & & & \\
\hline
\end{tabular}




\subsubsection{Entomotoxicology Results}

After spiking liver tissue with varying fentanyl concentrations $(0,10,100$, and $350 \mathrm{ug} / \mathrm{kg}$ ), we anticipate observing fentanyl and metabolites in the larva and pupal stages of the insects feeding on the spiked tissues. The presence of these drugs and metabolites is expected to create a difference in physical development (mass, length, and width) of the insects in comparison to the control groups. Insects were collected from the treatment groups after four and twelve days of allowing the insects to feed on the spiked human liver tissue. Five larvae and five pupae were randomly selected from each collection day for QuEChERS extraction of fentanyl and metabolites (Table 4.8). Larva samples were also analyzed in pools of four larva for triplicate analysis (Table 8). Individual larva and pooled larva were analyzed to determine the best course for sampling of insects for toxicology analysis. From the larvae collected on day four, fentanyl was detected in all the treatments except for the control liver tissue spike. The average concentrations $(n=5)$ for the single larvae collected at day four were: low treatment $1.3 \pm 0.4 \mu \mathrm{g} / \mathrm{kg}$, medium treatment $11.6 \pm 5.0 \mu \mathrm{g} / \mathrm{kg}$, and high treatment $26.9 \pm 12.8 \mu \mathrm{g} / \mathrm{kg}$. Fentanyl and norfentanyl were detected in the pooled larva samples $(n=4)$ with average concentrations $(n=3)$ for the low treatment at $1.6 \pm 0.3 \mu \mathrm{g} / \mathrm{kg}$, medium treatment at $18.4 \pm 2.2 \mu \mathrm{g} / \mathrm{kg}$, and high treatment at $31.5 \pm 2.4 \mu \mathrm{g} / \mathrm{kg}$ for fentanyl. Norfentanyl was detected in the medium and high treatments at $0.7 \pm 0.1 \mu \mathrm{g} / \mathrm{kg}$ and $1.4 \pm 0.2 \mu \mathrm{g} / \mathrm{kg}$, respectively. From the pupae collected on day twelve, fentanyl was only detected in the medium and high treatment groups. Average fentanyl concentrations for the pupae from the medium treatment were 0.14 $\pm 0.04 \mathrm{ug} / \mathrm{kg}$ and from the high treatment group $0.32 \pm 0.12 \mu \mathrm{g} / \mathrm{kg}$. Liver to larvae ratios were calculated for the day four larvae for low $7.5 \pm 2.2 \mu \mathrm{g} / \mathrm{kg}$, medium at $8.1 \pm 3.9 \mu \mathrm{g} / \mathrm{kg}$, and high at $9.1 \pm 3.8 \mu \mathrm{g} / \mathrm{kg}$. The average liver to larvae ratio between the three groups was $8.2 \pm 3.2 \mathrm{ug} / \mathrm{kg}$. A correlation plot for the liver to larvae concentration was generated for the day four data set. The correlation determined an $R^{2}=0.753$ for the liver to larvae concentration as a proof of concept. 
Table 4.8: Larvae and pupae fentanyl concentrations from day four and day twelve collections.

\begin{tabular}{|c|c|c|c|c|c|c|c|c|}
\hline \multicolumn{9}{|c|}{ Insect Concentrations } \\
\hline \multirow{2}{*}{ Compound } & \multicolumn{4}{|c|}{$\operatorname{Larvae}^{\mathrm{a}}(\mu \mathrm{g} / \mathrm{kg})$} & \multicolumn{4}{|c|}{$\operatorname{Pupae}^{\mathrm{b}}(\mu \mathrm{g} / \mathrm{kg})$} \\
\hline & Control $^{\mathrm{c}}$ & Low $^{\mathrm{c}}$ & Medium $^{\mathrm{c}}$ & High $^{\mathrm{c}}$ & Control $^{\mathrm{d}}$ & Low $^{\mathrm{d}}$ & Medium $^{\mathrm{d}}$ & $\operatorname{High}^{\mathrm{d}}$ \\
\hline 4-ANPP & ND & ND & ND & ND & ND & ND & ND & ND \\
\hline$\beta$-Hydroxyfentanyl & ND & ND & ND & ND & ND & ND & ND & ND \\
\hline Fentanyl & ND & $1.3 \pm 0.4$ & $11.6 \pm 5.0$ & $26.9 \pm 12.8$ & ND & ND & $0.14 \pm 0.04$ & $0.32 \pm 0.12$ \\
\hline Norfentanyl & ND & ND & ND & $<$ LLOQ & ND & ND & ND & ND \\
\hline \multirow{2}{*}{ Compound } & \multicolumn{4}{|c|}{ Pooled Larva $^{\mathrm{e}}(\mu \mathrm{g} / \mathrm{kg})$} & & & & \\
\hline & Control $^{\mathrm{c}}$ & Low $^{\mathrm{c}}$ & Medium $^{c}$ & $\operatorname{High}^{\mathrm{c}}$ & & & & \\
\hline 4-ANPP & ND & ND & ND & ND & & & & \\
\hline$\beta$-Hydroxyfentanyl & ND & ND & ND & ND & & & & \\
\hline Fentanyl & ND & $1.6 \pm 0.3$ & $18.4 \pm 2.2$ & $31.5 \pm 2.4$ & & & & \\
\hline Norfentanyl & ND & $<$ LLOQ & $0.7 \pm 0.1$ & $1.4 \pm 0.2$ & & & & \\
\hline
\end{tabular}

\begin{tabular}{l}
\hline \multirow{2}{*}{ Compound } \\
\cline { 2 - 3 } \\
\cline { 2 - 3 }
\end{tabular}

\subsubsection{Insect measurements}

Entire cohorts of each treatment were collected, and all insects were measured. Insect measurements from the two collection days and each of the four treatment groups were compiled and images were taken but due to single replication, no statistical significance could be drawn and the act was a function of practice for section 2 of this chapter.

Survivorship rates based on calculated eggs placed and insects collected on the two collection days were generated (Table 4.9). The high treatment group experienced the highest mortality at $71.8 \%$ 
mortality for day four collection and an $88 \%$ mortality rate for day twelve with only six total insects collected from the high treatment. The control group experienced a mortality rate of $21.7 \%$ and $25.6 \%$ for the day four and day twelve collections. The percentage of insects from egg placement to pupation ranged from $12 \%$ to $45 \%$ for the day twelve collection. The high treatment had the lowest percent pupated (12\%) of any of the treatment and control groups.

Table 4.9: Survivor rates for the day four and day twelve insect collections.

\begin{tabular}{cccc}
\hline & & & Day 4 \\
\hline \multirow{2}{*}{ Treatment $^{\mathrm{b}}$} & & Larvae & \\
\cline { 2 - 4 } & & & \\
\hline Congs Placed & Collected & Mortality $(\%)^{\mathrm{a}}$ \\
Low & 65 & 51 & 21.7 \\
Medium & 51 & 54 & 19.4 \\
High & 66 & 48 & 5.8 \\
\hline
\end{tabular}

Day 12

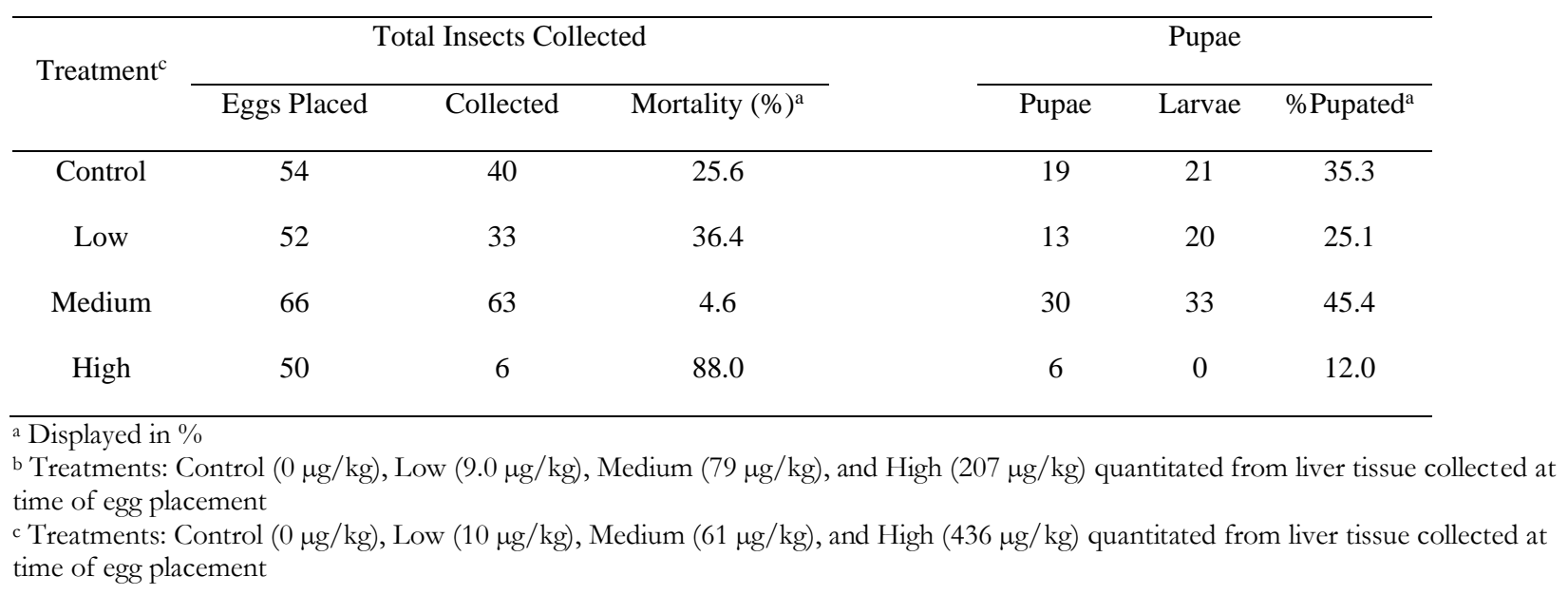

\subsubsection{Discussion}

\subsubsection{Validation}

Following the ASB Standard 036 requirements, a full validation was performed for both insect matrices. Both matrices were evaluated over six calibrators and linear regression analysis with a weight of $(1 / x)$ was deemed appropriate by evaluation of bias, precision, $R^{2}$ values, and residual plots over five runs. No interferences from blank matrix, target analytes themselves, or from commonly encountered 
analytes were identified. Ionization suppression or enhancement was within acceptable criteria for all analytes in both matrices at both concentrations evaluated. Overall, the pupa matrix appeared to be less impacted by ionization suppression or enhancement with percent ionizations under $\pm 9 \%$ for all analytes in both concentrations. The larvae extractions experienced more ionization effects at the low end compared to the high and to the pupa matrix. Norfentanyl for the larvae extractions experienced the most ionization enhancement $(17.9 \%)$ at the low concentration compared to all the other analytes in any concentration or matrix. Conversely, fentanyl experienced the most ionization suppression (-10.9\%) at the low concentration for the larva matrix. In comparison of the two insect matrices, pupae experienced less ionization suppression or enhancement, obtained greater recoveries, and maintained overall better process efficiencies (Table 4). The differences could be due to the developmental progress of the insect. It has been shown that necrophagous fly larvae have increased lipid development between day three and six of development with a decrease after day seven $(46,145)$. The increased lipid content combined with the metamorphosis occurring in the pupae could be contributing factors to the matrix effects and recoveries calculated. The bias and precision for both matrices were well within the required criteria with $\beta$-hydroxy fentanyl at the low concentration having the highest bias of $11.3 \%$ and all other analytes at the three concentrations were below $\pm 10.1 \%$. For autosampler stability, the larval and pupal extractions were stable for up to $72 \mathrm{hr}$ with no analyte exceeding $\pm 15.8 \%$ response change from time zero. Fentanyl and 4-ANPP experienced a decrease in response for both low and high concentrations over time and norfentanyl experienced an increase over time at low concentrations but a decrease at the high concentration. $\beta$-Hydroxyfentanyl experience a mixed result for stability with increases and decreases in concentration over time for both concentrations.

\subsubsection{Entomotoxicology}

From all three treatments, among the spike compounds, only fentanyl was detected in either the single-specimen larvae or pupae extractions. With the presence of metabolites not detected in the single 
insect extractions, the single insects most likely contained metabolites below our limit of detection. Although the quantifying transition for norfentanyl was observed in the high-concentration treatment, it did not meet the full criteria for acceptability. As expected, the percentage of larvae from each treatment with detectable fentanyl increased as the treatment concentration increased and the detection of norfentanyl in the pooled larva specimens. The treatment concentrations low $(10 \mu \mathrm{g} / \mathrm{kg})$, medium (100 $\mu \mathrm{g} / \mathrm{kg}$ ), and high (350 ug/ $\mathrm{kg}$ ) were based on the findings from Palamalai et al who presented findings of liver fentanyl concentrations in five non-drug related deaths ranging from 11-104 $\mu \mathrm{g} / \mathrm{kg}$ with a mean of $38 \mu \mathrm{g} / \mathrm{kg}$; liver fentanyl concentrations in 26 mix drug related deaths ranging from $6-235 \mu \mathrm{g} / \mathrm{kg}$ with a mean of $80 \mu \mathrm{g} / \mathrm{kg}$; and liver fentanyl concentrations in 33 fentanyl-related deaths ranging from 18-365 $\mu \mathrm{g} / \mathrm{kg}$ with a mean of $104 \mu \mathrm{g} / \mathrm{kg}$ (64). The low treatment corresponds to a low dose of fentanyl and not necessarily related to cause of death, the medium is close to the mean of the mixed drug and fentanyl related deaths and the high corresponds to the highest concentration reported by Palamai et al (64). Mclntyre et al presented data from 87 cases for liver fentanyl concentrations ranging from 6.9-689 $\mu \mathrm{g} / \mathrm{kg}$ with a mean of $93.3 \mu \mathrm{g} / \mathrm{kg}$, indicative of the wide range of fentanyl concentrations detected in liver tissue (115).

Detection of drugs in insects can be vital in corroborating traditional toxicology matrices or as the only matrix available in cases with advanced decomposition, exsanguination, or burn victims $(39,58,59)$. Beyer et al. presented a case of suicide by overdose with phenobarbital where the body was badly decomposed with no fluids or organs to test for drugs (58). The deceased had been last seen 14 days prior to discovery and the remains were badly decomposed with the head, thorax, and abdomen completely skeletonized (58). The decedent had a history of suicide attempts, a recent prescription for 100 tablets of phenobarbital, and the bottle was found empty in her purse (58). Analysis and detection of phenobarbital in the larva collected from the cadaver was the only toxicology results available for corroborating the physical evidence found on the deceased (58). In a case presented by Bugelli et al., benzodiazepines and 
metabolites were detected in the burnt cadavers of a maternal filicide-suicide by fire (59). In this case, the detection of drugs in the insects was used to corroborate the findings of drugs in the fluids and tissues collected (59). When there is a death with a delayed recovery like suicide or drug overdose, advanced decomposition of the body can occur to the point where insect tissue is the only matrix available or can corroborate the findings of any more traditional matrix available (39).

The ratio of larvae concentration to liver concentration for each of the three treatments were within a single standard deviation and the mean ratio between the groups $8.2 \pm 3.2 \mu \mathrm{g} / \mathrm{kg}$ incorporated all three mean ratios for the treatments. The simple linear regression of the liver to larva concentration generated $a R^{2}=0.753$ which indicates that $75.3 \%$ of the variation of the larva fentanyl concentration can be predicted by the concentration of fentanyl in the liver. This limited correlation is similar to the findings of El-Samad et al. that found correlation of larvae collected from rabbits dosed with tramadol (45). In a dissertation, Peace et al. presented correlation $\mathrm{R}^{2}$ values ranging from 0.7727 to 0.9976 for barbital, phenobarbital, pentobarbital, and thiopental for larvae concentrations to food source with the conclusion of strong correlation (40). A moderate to strong correlation is needed to be able to draw quantitative conclusions for interpreting human tissue concentration based on insect concentration. However, as both current study and the El-Samad et al., have low number of replicates, stronger models may permit a mathematical correlation between drug concentration within insect tissues and in the original human tissue (45). Ideally, authentic tissue samples from authentic overdose casework with paired known blood concentrations would be best for expansion for fentanyl relationship of insect to tissue concentrations as well as expansion to at least three replications for statistical relevance and increased power of the test. Of particular interest is recovery of fentanyl from pupae from the medium and high-concentration treatment livers, which displays the persistence of this drug across the immature life stages of $L$. sericata. It is consistent with successful extraction of methadone from puparia, the remnant casing in which the pupa develops, derived from the larval integument (44). Nevertheless, the concentrations of fentanyl 
detected in the pupa tissue were much lower than the concentrations quantitated from the larvae (Table 8). The detection of fentanyl in lower concentrations in the pupae compared to the larvae indicates that although the drug is persistent, the immature fly is able to eliminate fentanyl as it progresses through its life stages. Metabolism of fentanyl is supported by the detection of norfentanyl in the pooled larval samples. Norfentanyl was not part of the opioid spike mix, nor was it detected in the homogenized liver samples taken at day 4 or day 12 . Therefore, the only source of norfentanyl in the pooled samples must have been the larvae themselves. Opioid metabolism has previously documented for heroin, methadone, and codeine in various blow fly species $(44,103,144)$. In the better-studied human system, fentanyl is metabolized predominantly by cytochrome P450 isoforms (102). Cytochrome P450 monooxygenases have been identified in Lucilia cuprina (Wiedemann) (Diptera: Calliphoridae) larva, where they are implicated in organophosphate insecticide resistance in both L. cuprina and L. sericata (150-153). These monooxygenases are likely to be the main path for metabolism in the insect and metabolic pathways for the monooxygenases encourage biotransformation of drugs to less hydrophobic or more hydrophilic compounds for excretion $(44,102)$. In blow fly larvae, water soluble or less hydrophobic molecules are excreted out of the hemolymph via Malpighian tubules $(44,154)$. Given the observed reduction of fentanyl concentration in the collected pupal samples and lack of detectable metabolites, it appears the insects were able to eliminate the drug effectively, but incompletely, as fentanyl was still detected at the higher treatments.

\subsubsection{Insect development}

The fentanyl produced observable development changes to the larval growth. The mass, length, and width of the low and medium treatment groups were visibly larger than the control and high treatment groups. These results are consistent with previous studies on blow fly larvae exposed to ketamine, methamphetamine, and tramadol $(45,46,49)$. Ordinarily, insects trade-off increased body size with increased development time (155). However, in this experiment, 19 of 40 collected individuals (47\%) 
in the control group had already pupated by day 12 . In the low-concentration treatment, $13 / 33(39 \%)$ had pupated, and in the medium-concentration treatment, 30/63 (47\%) had pupated (Table 4.9). These results would seem to suggest the paradoxical response that for L. sericata, low concentrations of opioids may actually increase the growth rate of the larval stage without a concurrent increase in its duration. Opioid exposure does show a dose-response on development, as seen in the small number of survivors in the high-concentration treatment. Although the surviving individuals reached the same body size as the control group, only six of the original 50 eggs placed on the high treatment for day twelve survived to collection. Among pupal treatments, the only significant difference in physical characteristics was a slightly increased pupae length in the low concentration treatment compared to the medium and control groups. Lower number of specimens combine with a high variance did not allow for a significant difference in physical characteristics. The combined issues of high mortality and low number of pupated insects creates the need for further work with more insects being placed and more replicates to fully evaluate the later life stages. Estimation of ${ }_{\min } \mathrm{PMI}$ can impact an investigation or cause of death and the impact of drugs on the growth of insects is an important factor to consider when using an entomological based PMI estimate.

\subsubsection{Conclusion}

Presented in this study is a full validation following the ASB Standard 036 requirements for a QuEChERS extraction of fentanyl and metabolites from larval and pupal tissue. The method met all the criteria mapped out by the ASB and was applied to authentic insect samples that were reared on human tissue containing fentanyl. Fentanyl was detected in the larvae and pupae that were collected from the human liver tissue. A moderate positive correlation for the liver to larvae concentrations was calculated and provides hope that larvae can be used in the future to, at minimum, determine the presence of fentanyl from feeding on human tissue and potentially estimate human tissue concentrations. The fentanyl infused tissue had some effects on the development of the insects including an increase in larval 
mass, length, and width at low concentrations. At high concentrations the insects experienced a high mortality rate alluding to low concentrations of fentanyl having a positive effect on the insect but a negative effect at higher concentrations. Due to the high mortality rates at the high concentration, more replicates are needed to fully evaluate the growth effects of fentanyl in the later life stages of the insects.

Overall, the QuEChERS extraction was successful at extracting fentanyl from larvae and pupae collected from feeding on human liver tissue that contained fentanyl. This provides a method for detecting and quantifying fentanyl in an alternate matrix, liver to larvae concentrations, and shows the persistence of fentanyl through the insect life cycle to pupation.

\subsection{Section 2: Physical Effects of Fentanyl on Larva and Pupa (Lucilia sericata)}

\subsubsection{Overview}

This section presents the morphometric comparisons for the larva and pupa reared in task 2.a. To determine the effects of fentanyl on the growth and development of the blow flies, the mass, and percent stage (first instar, second instar, third instar, or pupa) of the insect were statistically compared using nested ANOVA to determine if the control and treatment groups differ. Further Tukey-HSD test determined which treatments are significantly different and due to sample size differences, restricted maximum likelihood (REML) was applied. These statistical tests determined if the drug treatments have any significant effects on the insect's development. Age estimations were also performed using the mean length for the larval stages on collection day. Maximum larval length for each treatment on days collected were also presented.

Since entomological techniques utilize the growth and development of insects to estimate the minimum time since death, it is crucial to understand the role of fentanyl in the development of Lucilia sericata blow flies. Insects follow a predictable life cycle that xenobiotics can influence. While several drugs have been explored in entomotoxicological studies, little is known about the direct effects that fentanyl may pose on the growth and development of blow fly larvae. 


\subsubsection{Morphometrics}

For an entomologist to estimate the $\min P M I$, the age of the oldest insects collected need to be estimated $(2,17)$. This is typically accomplished in two ways: by identification of the insect life stage or by estimation based on larval size which is usually length (17). The first age estimation method requires the identification of the discrete and distinguishable stages of development that blow fly larva develop through and the thermal history where the specimen developed $(2,17)$. This is often referred to as a thermal summation model and uses linear regression to analyze the positive relationship of the insect's growth and temperature (2). An insect's growth rate and development is driven by temperature and this allows for a predictable growth rate $(2,17)$. Using this method, the development is measured as physiological time in units of degree days or degree hours (2). The discrete life stages for the insect require a certain amount of accumulated degree hours (ADH) to progress to the next stage of development and by documenting the thermal summation of degree hours necessary for an insect to reach the stage at the moment of collection a $\min P M I$ can be estimated (2).

The second method to estimate age is by measurement of larval size, most commonly larval length $(17,33)$. This method relies on growth data from the species of the insect over different temperatures $(8,17,23,37)$. Once a larva hatches from its egg, it will steadily and predictably grow until it reaches pupation $(17,23)$. The larva continuingly grow in length as they feed and progress through their larval stages of first, second, and third instar until they stop feeding at which they reach their peak length $(2,37,156)$. During the development time from third instar to pupa, the larva change in behavior by stopping feeding and migrating or wandering from the feeding substrate $(21,33)$. This generates a nonlinear relationship for length to time for the larval stage as the growth rate while the larva are wandering slows and the larva length decreases (37). Popular ways to calculate age based on length of larva include growth curves, isomegalen, or isomorphen diagrams $(2,8,17,37,156)$. For growth curves, the length of the larval insect is the dependent variable plotted against time as the independent. With the literature 
curve data, the length of a collected larvae can be used to estimate an age of the insect if the temperature is known $(37,156)$. For these curves, a polynomic equation can be generated to fit the curve and with the length inputted, a time can be estimated (37). An isomegalen-diagram plots the time since hatching against temperature with lines drawn for identical larval lengths $(2,156)$.

Drugs or toxins have been presented in literature to impact the growth of insects in terms of temporal growth, life stage, and physical characteristics $(39,46,157)$. Due to the desire for toxicological analysis, the focus of this study is on the impacts of fentanyl on the life stage and morphometrics in terms of how they would impact the estimation of ${ }_{\min } \mathrm{PMI}$ rather than the raw temporal growth.

\subsubsection{Methods}

\subsubsection{Design}

Fentanyl was spiked into $200 \mathrm{~g}$ aliquots of human liver homogenate to evaluate four concentrations: control $(0 \mu \mathrm{g} / \mathrm{kg})$, low $(10 \mu \mathrm{g} / \mathrm{kg})$, medium $(100 \mu \mathrm{g} / \mathrm{kg})$, and high $(350 \mu \mathrm{g} / \mathrm{kg})$ concentrations. For replicate \# 3, an authentic postmortem liver specimen with a fentanyl concentration of $111 \mu \mathrm{g} / \mathrm{kg}$, norfentanyl concentration of $8.1 \mu \mathrm{g} / \mathrm{kg}$, and a 4-ANPP concentration of $5.8 \mu \mathrm{g} / \mathrm{kg}$ was homogenized to be treated as a fifth treatment group. The $200 \mathrm{~g}$ treatment portions were aliquoted as seven $25 \mathrm{~g}$ aliquots onto aluminum foil sheets inside 8 oz plastic containers (Figure 4.3) with sand as a substrate for pupation. The center of the tops of the containers were cut out and a piece of cloth was placed under the top, covering the container to allow airflow while preventing the escape of the insects. To each aliquot of liver, approximately 70-90 eggs (by mass) were placed and allowed to feed undisturbed until time of collection (Figure 4.5). The containers were housed in the Percival I36LLVLC8 incubator at 25 ${ }^{\circ} \mathrm{C}\left( \pm 0.5^{\circ} \mathrm{C}\right)$ at $65 \%$ relative humidity $( \pm 10 \%)$ with a $12: 12$ (L:D) photoperiod. Insects were collected at seven sampling times: at 48 hours (day 2), 72 hours (day 3), 96 hours (day 4), 120 hours (day 5), 144 hours (day 6), pupa (192 hours or day 10), and emergence (day 21) (Figure 4.4). Larvae were collected and heat killed with boiling water for $90 \mathrm{~s}$ and rinsed with methanol before stored at $-20{ }^{\circ} \mathrm{C}$ with no liquid 
preservatives added $(7,40,46)$. Collected pupae were rinsed with deionized water and then methanol before being stored at $-20^{\circ} \mathrm{C}$ with no addition of any liquid preservative. No preservative was chosen due to the larval cuticle acting as a semipermeable membrane which can allow water or ethanol to diffuse into the body cavity and potential to leech out the target analytes into the preservative solution (149). Liver samples were collected at the time of egg placement (day 0 ) and at the day of collection to establish the initial tissue concentrations and to evaluate any metabolism of the drugs by the feeding media. This protocol was repeated for three replicates approximately 6-8 weeks apart. The colony was described in section 1 of this chapter (4.1.3). For replicate \#1, the colony was at generation 24 , for replicate \#2 the generation was 26 , and for replicate \#3 the generation was 28 .

To evaluate developmental effects, the mass $(\mathrm{mg})$, length $(\mathrm{mm})$, and width $(\mathrm{mm})$ of the collected insects were documented (Figure 4.4). Metrics also evaluated were survivor rates, percent stage, and percent pupated. Statistical analysis for the physical characteristics including nested ANOVA and TukeyKramer HSD were performed using JMP Pro 15.1.0.

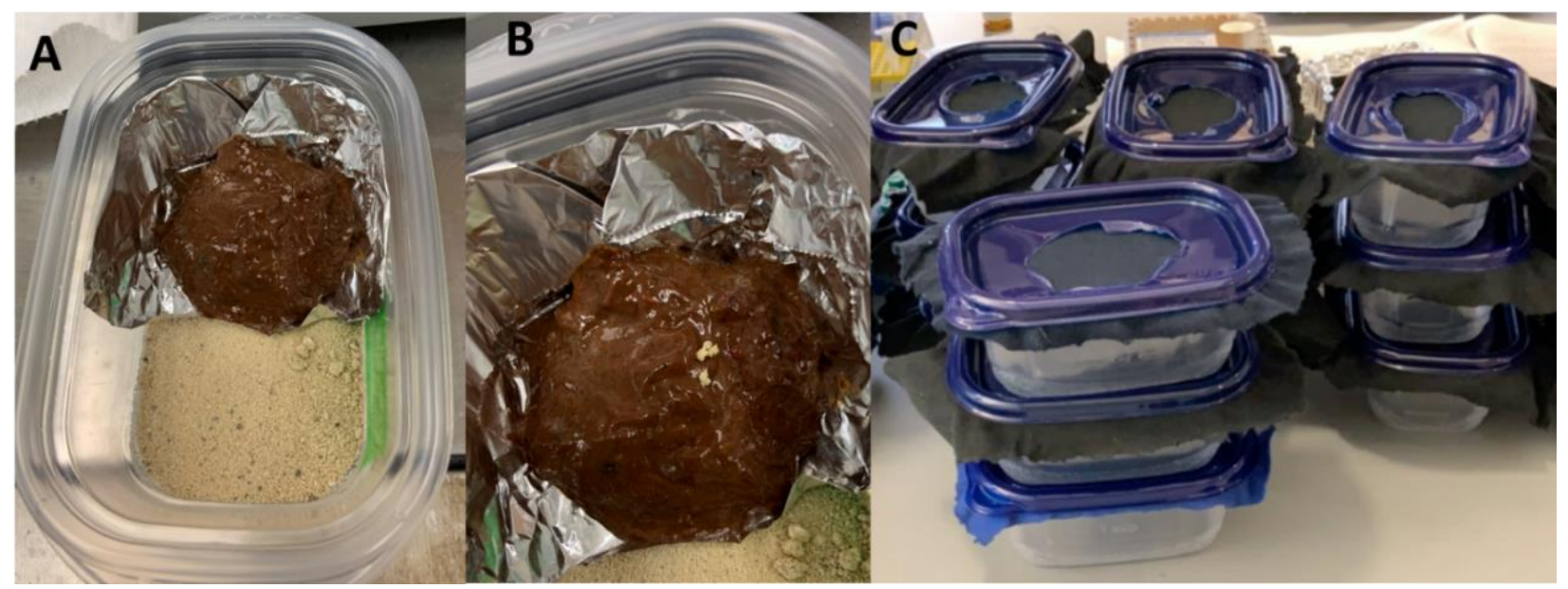

Figure 4.3: Fortified liver ( $25 \mathrm{~g}$ ) aliquot $(\mathrm{A})$ with 70-90 eggs placed (B). 


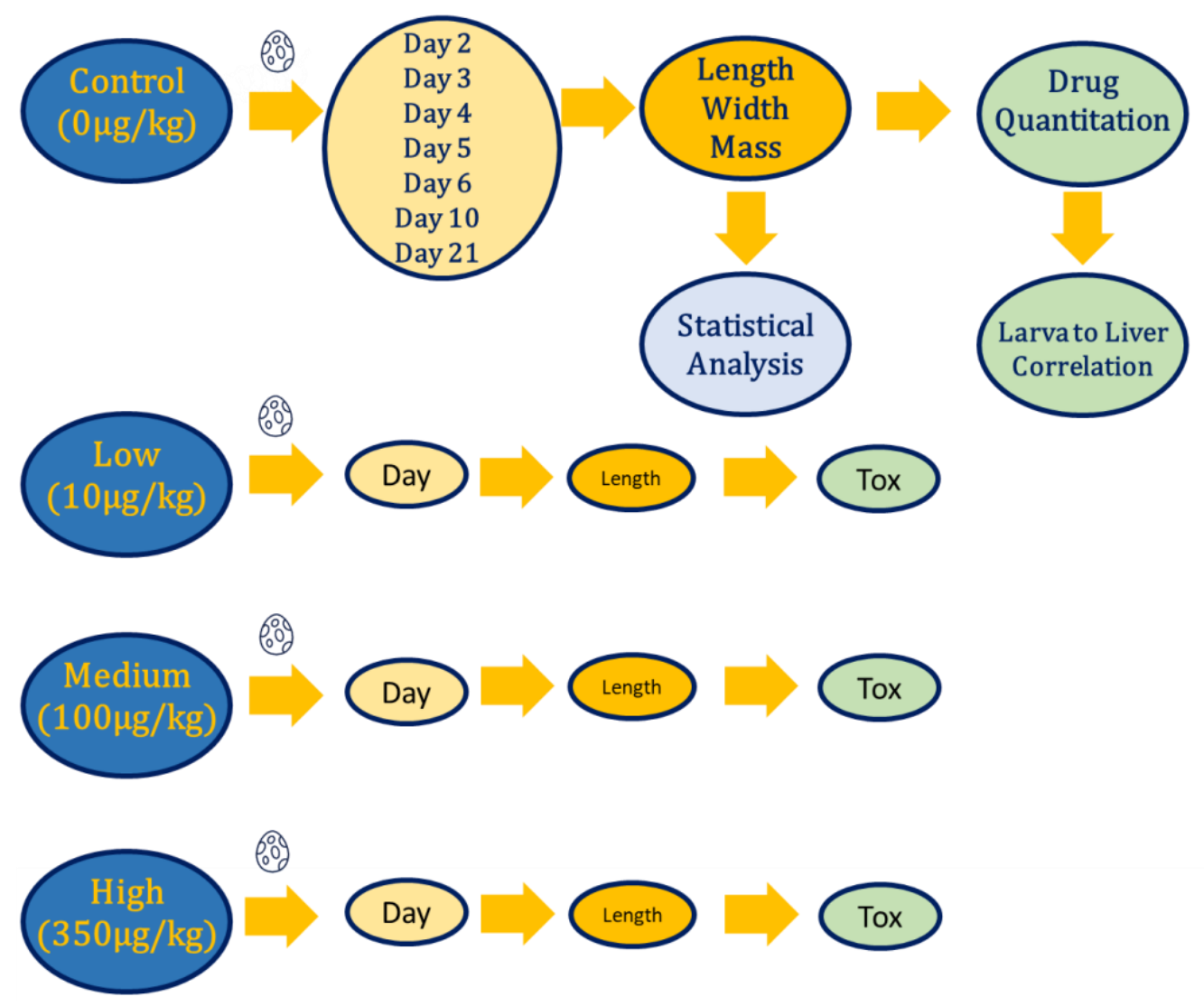

Figure 4.4: Schematic of the design workflow.

\subsubsection{Results}

Entire cohorts of each treatment were collected, and all insects were measured. For the statistical analysis of the groups, mean was selected as the measure for length, width, and mass of the insects. In the figures below, nested ANOVA ( $\alpha=0.05$ ) followed by Tukey-HSD statistical tests were performed with the letters A, B, and C representing significant difference. Treatments not connected by a letter are considered significantly different from each other. By the nesting of the replicates and days, statistical comparisons were performed for each treatment within replicate.

Table 4.10 shows the percent stage for the insects collected at each day by life stage. Small differences can be observed but the percent stage for each treatment was not significantly different. The 
authentic or validation set is only a single replicate so no statistical comparisons can be made but the percentages are displayed.

Table 4.10: Percent stage over time for each life stage $\left(2^{\text {nd }}\right.$ instar larva, $3^{\text {rd }}$ instar larva feeding, $3^{\text {rd }}$ instar larva wandering, pupa, and adult flies).

\begin{tabular}{|c|c|c|c|c|c|c|c|c|c|c|c|c|c|}
\hline & \multicolumn{2}{|c|}{$2^{\text {nd }}$ Instar } & \multicolumn{3}{|c|}{$3^{\text {rd }}$ Instar Feeding } & \multicolumn{5}{|c|}{$3^{\text {rd }}$ Instar Wandering } & \multicolumn{2}{|c|}{ Pupa } & \multirow{2}{*}{$\frac{\text { Adults }}{\text { Day } 21}$} \\
\hline & $\begin{array}{c}\text { Day } \\
2\end{array}$ & $\begin{array}{c}\text { Day } \\
3\end{array}$ & $\begin{array}{c}\text { Day } \\
3\end{array}$ & $\begin{array}{c}\text { Day } \\
4\end{array}$ & $\begin{array}{c}\text { Day } \\
5\end{array}$ & $\begin{array}{c}\text { Day } \\
4\end{array}$ & $\begin{array}{c}\text { Day } \\
5\end{array}$ & $\begin{array}{c}\text { Day } \\
6\end{array}$ & $\begin{array}{c}\text { Day } \\
10\end{array}$ & $\begin{array}{c}\text { Day } \\
21\end{array}$ & $\begin{array}{c}\text { Day } \\
10\end{array}$ & $\begin{array}{c}\text { Day } \\
21\end{array}$ & \\
\hline Cntrl $^{\mathrm{a}}$ & 100 & 0 & 100 & 28.1 & 0.0 & 71.9 & 100 & 100 & 19.4 & 7.8 & 80.6 & 19.3 & 73.0 \\
\hline Low $^{\mathbf{a}}$ & 100 & 0 & 100 & 35.8 & 0.5 & 64.2 & 99.5 & 100 & 45.2 & 5.9 & 54.8 & 19.5 & 74.6 \\
\hline Med $^{\mathbf{a}}$ & 100 & 0 & 100 & 30.1 & 0.0 & 69.9 & 100 & 100 & 26.6 & 4.2 & 73.4 & 11.6 & 84.2 \\
\hline High $^{\mathbf{a}}$ & 100 & 0 & 100 & 41.7 & 0.0 & 58.3 & 100 & 100 & 21.1 & 8.9 & 78.9 & 28.2 & 62.9 \\
\hline Auth $^{b}$ & - & 0 & 100 & 88.0 & 58.8 & 12.0 & 41.2 & 100 & 31.4 & 20.3 & 68.6 & 10.1 & 79.7 \\
\hline
\end{tabular}

a Total of all three replicates $(n=3)$

b The authentic or validation set represents only one replicate of data $(n=1)$

\subsubsection{Mixed Effects Model}

For the mixed effects model, the equation below was used.

$$
\begin{aligned}
\widetilde{\boldsymbol{Y}}=\widetilde{\boldsymbol{\mu}}+\delta_{j}+\tau_{i} & +(\delta \tau)_{j: i}+\left(\begin{array}{ccc}
1 & 0 & 0 \\
0 & 1 & 0 \\
0 & 0 & 1
\end{array}\right)\left(\begin{array}{c}
\tau_{i: L} \\
\tau_{i: M} \\
\tau_{i: W}
\end{array}\right)+\left(\begin{array}{ccc}
1 & 0 & 0 \\
0 & 1 & 0 \\
0 & 0 & 1
\end{array}\right)\left(\begin{array}{c}
\delta_{j: L} \\
\delta_{j: M} \\
\delta_{j: W}
\end{array}\right)+\left(\begin{array}{ccc}
1 & 0 & 0 \\
0 & 1 & 0 \\
0 & 0 & 1
\end{array}\right)\left(\begin{array}{c}
(\delta \tau)_{j: i: L} \\
(\delta \tau)_{j: i: M} \\
(\delta \tau)_{j: i: W}
\end{array}\right) \\
& +\widetilde{\mathbf{E}_{\boldsymbol{\rho}}}+\widetilde{\mathbf{E}_{\boldsymbol{u}}}
\end{aligned}
$$

In the equation, $Y_{L}, Y_{M}, Y_{W}$ are the dependent variables measured on the larva. Collectively, they

can be represented as $\widetilde{\boldsymbol{Y}}=\left(\begin{array}{c}Y_{L} \\ Y_{M} \\ Y_{W}\end{array}\right)$. Let $\mu_{L}, \mu_{M}, \mu_{W}$ be the mean length, mass, and width of the population of larva. Collectively, they can be represented as $\widetilde{\boldsymbol{\mu}}=\left(\begin{array}{l}\mu_{L} \\ \mu_{M} \\ \mu_{W}\end{array}\right)$. The Greek letters with $i$ and $j$ subscripts are linear coefficients for categorical variables (i.e., factors) where the subscript represents the level of the 
factor: $\tau$ (treatment), $\delta$ (day), and $\rho$ (replicate). Where $\widetilde{\mathbf{E}_{\boldsymbol{\rho}}} \sim M N V\left(0, \Sigma_{\rho}\right)$ represents the random effect due to replicates, and $\widetilde{\mathbf{E}_{\boldsymbol{u}}} \sim M N V\left(0, \Sigma_{\mathrm{u}}\right)$ represents random errors.

The mixed effects model using this equation, did not identify a trend or statistical difference in the means for the treatment groups $(\alpha=0.05)$. As shown in Figure 4.5, the replicates had variations too great for the treatment groups to overcome. Potential reasons for this include the natural variation of the insects, the variations in the liver tissues selected for the project, and the variation of the different generations of the insects. However, within replicate differences were observed and discussed in later sections. In Figure 4.5, the high treatment groups (purple) were higher than the control groups (orange) within each replicate, however they did not exceed the predicted means for the treatments. This indicates that the variations of the of the replicates was greater than the differences between the high treatment and control group for the entire model.

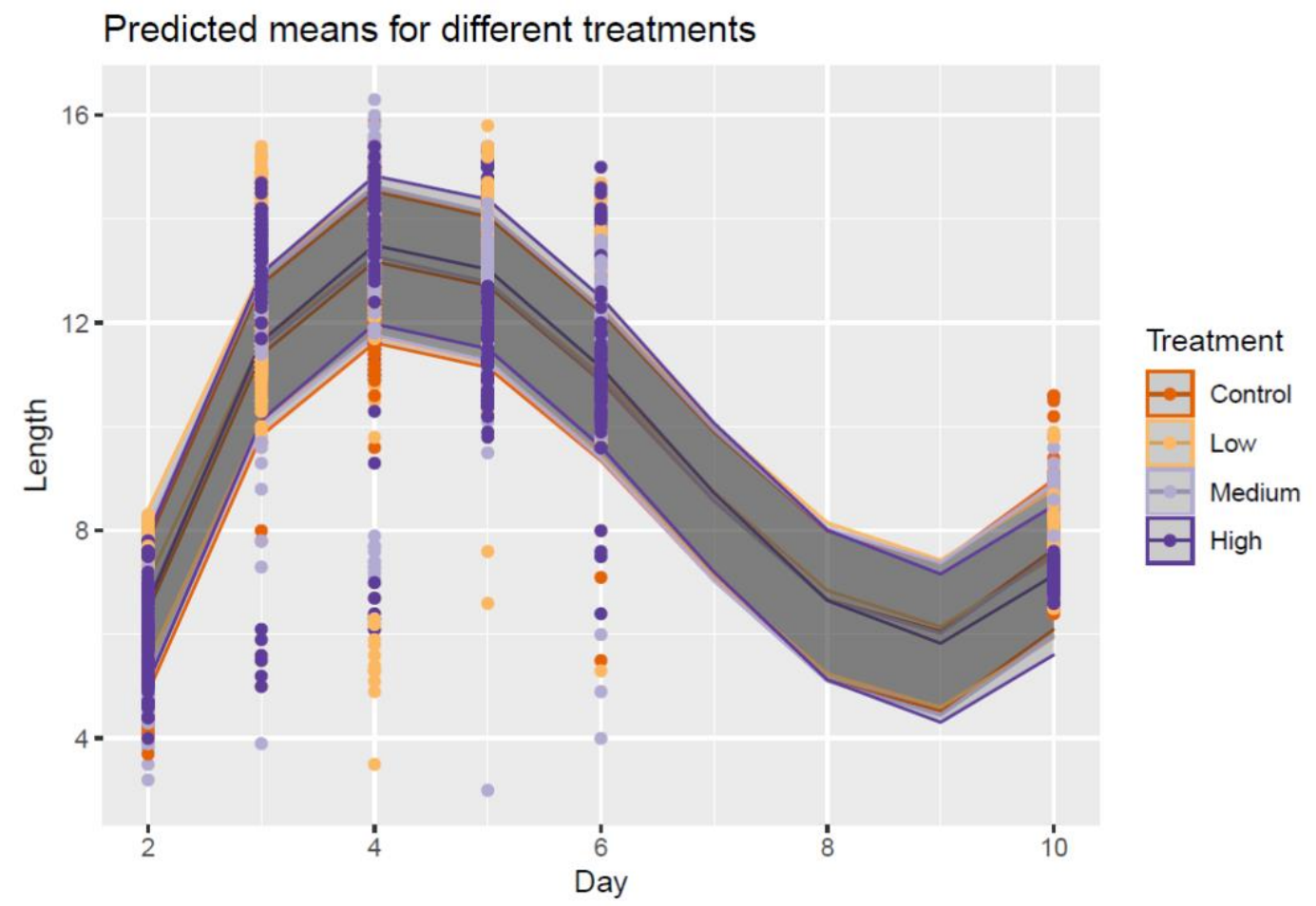

Figure 4.5: Plot of predicted means via the mixed effects model. 


\subsubsection{Mean Lengths Day 2}

For day 2 replicate $\# 1$ and \#2, there was no significant difference between the control and treatment groups for length (Figure 4.6). For day 2 in replicate \#3 (C), the low treatment group was significantly longer than the other groups $\left(t_{12.7,48.3}=1.93, p=0.0281\right)$.

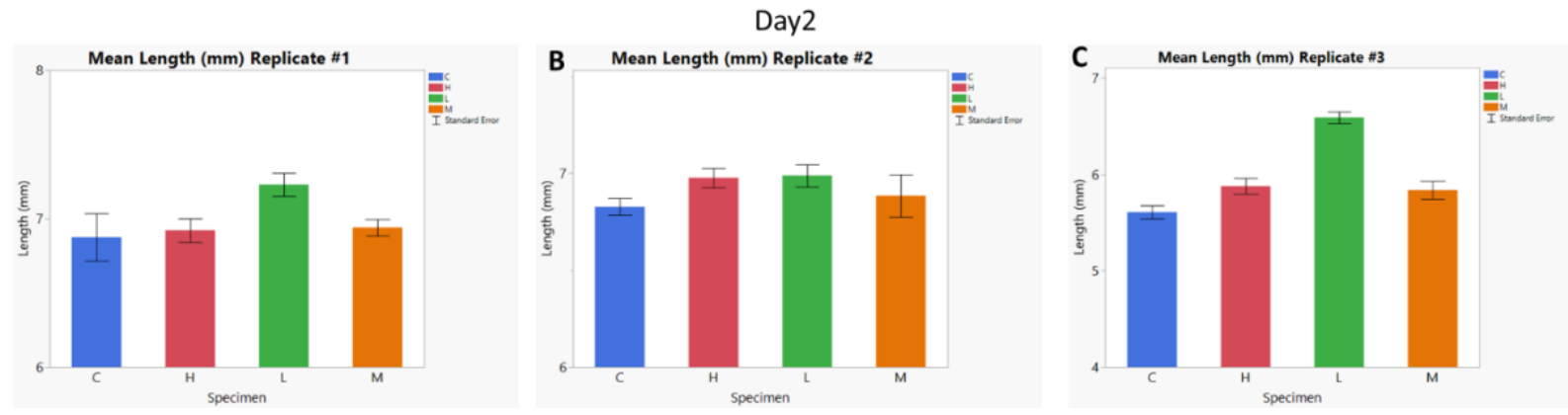

Figure 4.6: Comparison of mean lengths for insects collected on day 2 in replicates \#1-3.

\subsubsection{Mean Lengths Day 3}

For the insects collected on day 3 in replicate \#1 for the length (A), the low treatment was significantly longer than the other three treatment groups $\left(t_{12.7,51.6}=2.62, p=0.0116\right)$ (Figure 4.7). For replicate \#2 (B), the low treatment group was significantly longer than the other three groups $\left(\mathrm{t}_{12.7,51.6}=\right.$ $2.62, p=0.0011$ ). Also for replicate \#2, the medium group was significantly shorter than the control group $\left(t_{12.7,50.4}=-2.44, p=0.0181\right)$. For replicate \#3 for the insects collected on day $3(C)$, the low and control treatment groups were not significantly different. The medium and high treatment groups were significantly longer than the low and control groups $\left(t_{12.7,50.7}=3.18, p=0.0025\right)$. 


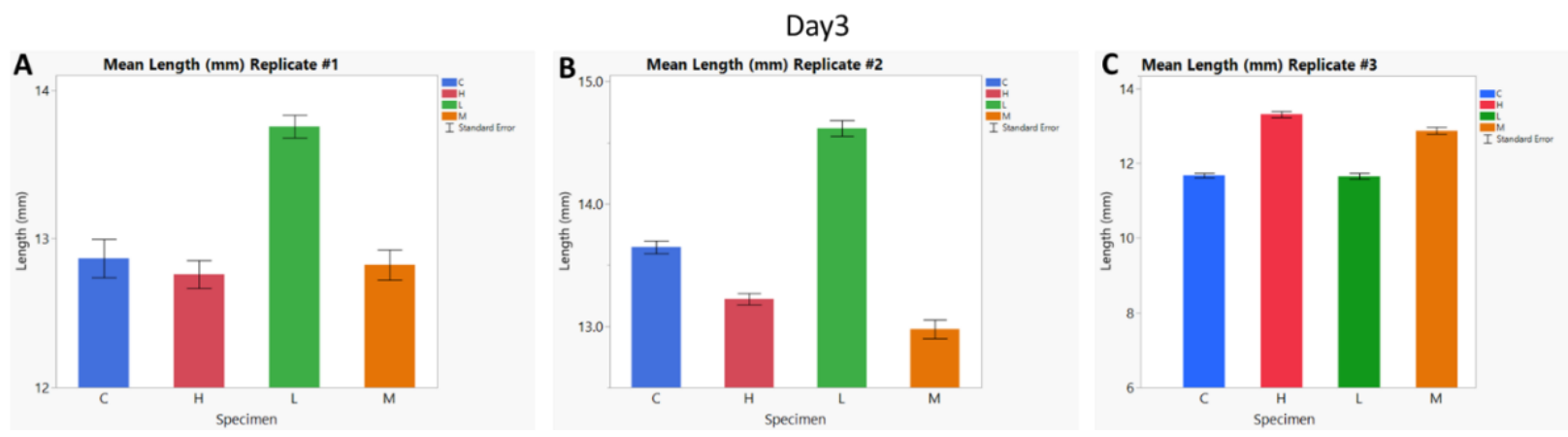

Figure 4.7: Comparison of mean lengths for insects collected on day 3 for replicates \#1-3.

\subsubsection{Mean Lengths Day 4}

For the larva collected on day 4 in replicate \#1 (A), the mean lengths for the low and high treatment groups were significantly lower than the control and medium groups $\left(t_{12.7,50.3}=-2.14, p=\right.$ 0.0376) (Figure 4.8). The medium treatment group had a mean length significantly longer than the other groups $\left(t_{12.7,52.4}=3.16, p=0.0026\right)$. For the larva collected on day 4 in replicate $\# 2(B)$, the mean length of the medium treatment group was significantly longer than the other three groups. For the insects collected on day 4 in replicate \#3 (C), the high and medium treatment lengths were significantly longer than the control group $\left(t_{12.7,50.9}=2.40, p=0.0201\right)$.

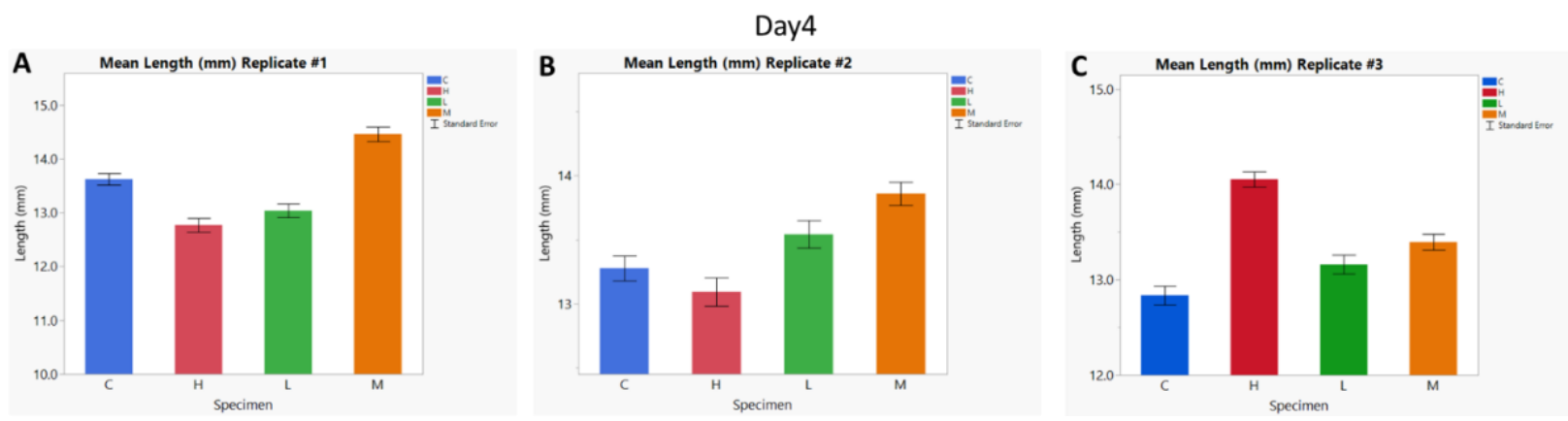

Figure 4.8: Comparison of mean lengths for insects collected on day 4 for replicates \#1-3. 


\subsubsection{Mean Lengths Day 5}

For mean length of the larva collected on day 5 in replicate \#1 (A), the high treatment group was significantly longer than the other three groups $\left(t_{12.7,50.7}=3.98, p=0.0002\right)$ (Figure 4.9). For the larva collected on day 5 in replicate \#2 (B), the mean length of the high treatment was significantly higher than the other three groups $\left(t_{12.7,52.4}=3.62, p=0.0007\right)$. For the insects collected on day 5 in replicate \#3 $(C)$, the mean length for the low treatment group was significantly longer than the control group $\left(\mathrm{t}_{12.7,51.1}=\right.$ $3.82, p=0.0004)$ and the high treatment was significantly shorter than the control group $\left(t_{12.7,50.3}=-2.54\right.$, $p=0.0144)$.

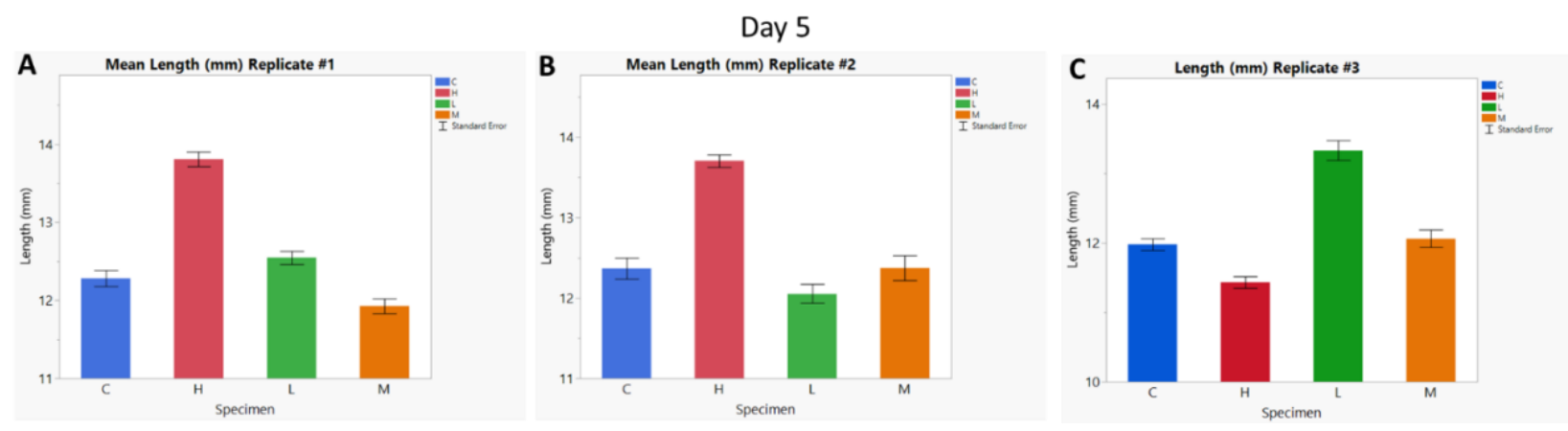

Figure 4.9: Comparison of mean lengths for insects collected on day 5 for replicates \#1-3.

\subsubsection{Mean Lengths Day 6}

For length of the larva collected on day 6 in replicate \#1 (A), there was no significance difference observed between the four treatments for mean length (Figure 4.10). For the larva collected on day 6 in replicate \#2 (B), there was no significance difference observed between the four treatments for mean length. For length of the larva collected on day 6 in replicate \#3 (C), there was no significance difference observed between the four treatments for mean length. 


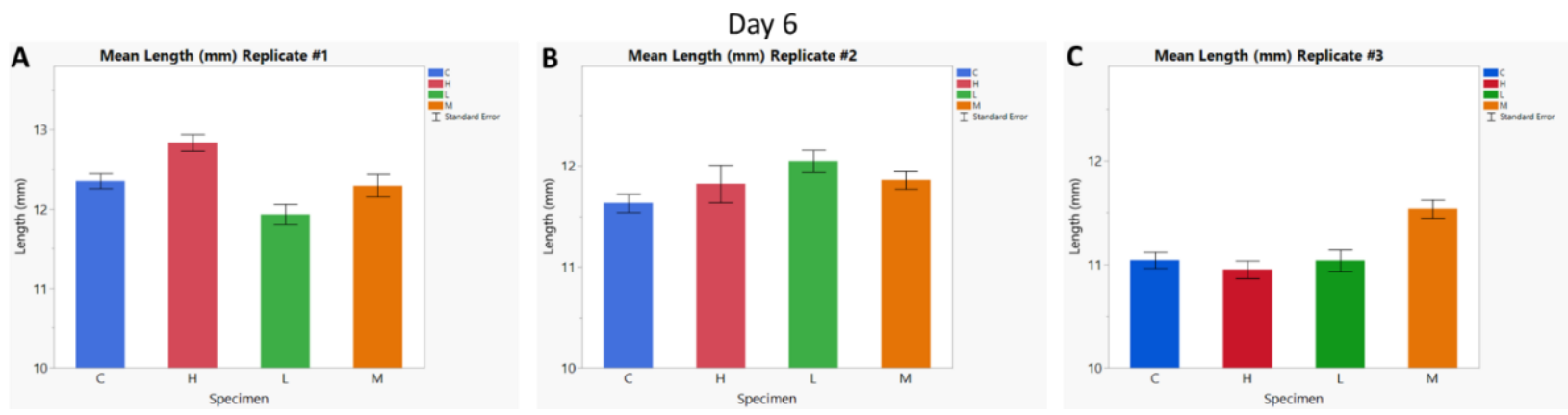

Figure 4.10: Comparison of mean lengths for insects collected on day 6 for replicates \#1-3.

\subsubsection{Mean Lengths Day 10}

For length of the pupa collected on day 10 in replicate \#1 (A), no significant difference was observed for the treatments compared to the control group (Figure 4.11). For the pupa collected on day 10 in replicate \#2 (B), the mean length for the high treatment group was significantly shorter than the other groups $\left(t_{12.7,51.6}=-2.30, p=0.0249\right)$. For the pupa collected on day 10 in replicate \#3 (C), no significant difference was observed for the treatments compared to the control group.

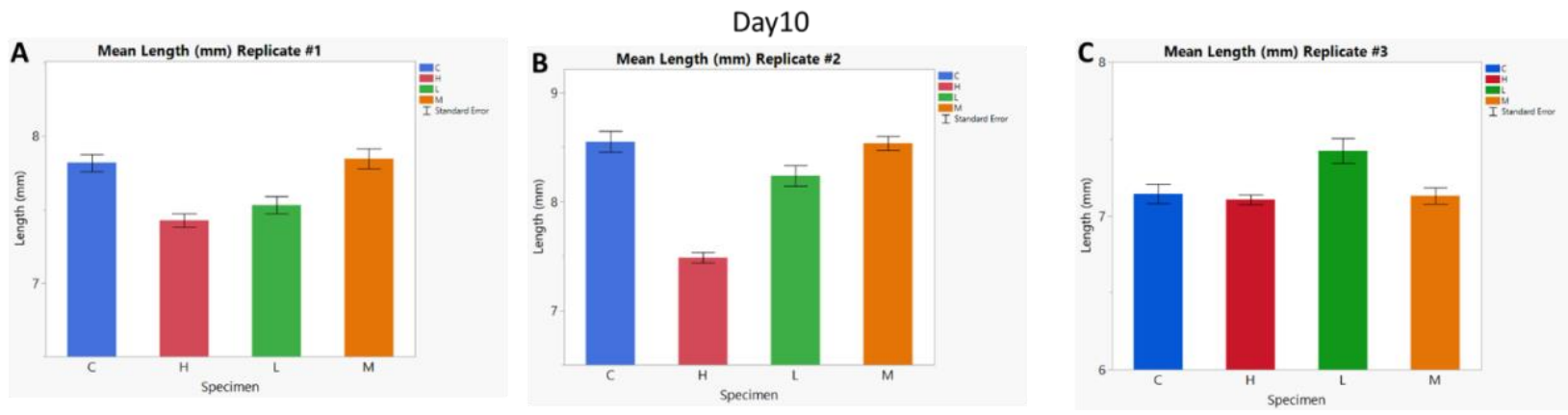

Figure 4.11: Comparison of mean lengths for insects collected on day 10 for replicates \#1-3. 


\subsubsection{Growth Curves}

The mean lengths for the control group for days 2 to 6 for each replicate (1-3) and the total comparison were plotted and a polynomial regression was applied. The simulated equation and resulting $R^{2}$ value are presented in Table 4.11. Using the mean length for each treatment, the simulated equation was used to calculate the estimation of the age of the larval cohort with time in days (Table 4.12). The estimated age was compared to the day collected or ground truth for the age of the insect and is presented in Table 4.12. A positive difference indicates an increase in estimated age compared to the actual age and a negative number indicates a decrease in estimated age compared to the actual age. A positive number also indicates a faster development rate and a negative number indicates a delay in development. Polynomic equations for age estimation do have limitations, including lengths beyond the asymptote of the equation and the late stage larva are much harder to predict with these models due to the nature of the insect growth. The polynomic equation cannot estimate age based on a length beyond its asymptote, therefore extraordinarily longer insects could produce inaccurate estimations and insects that do not pupate for any reason could have a much more inaccurate age estimation.

\subsection{Replicate \#1}

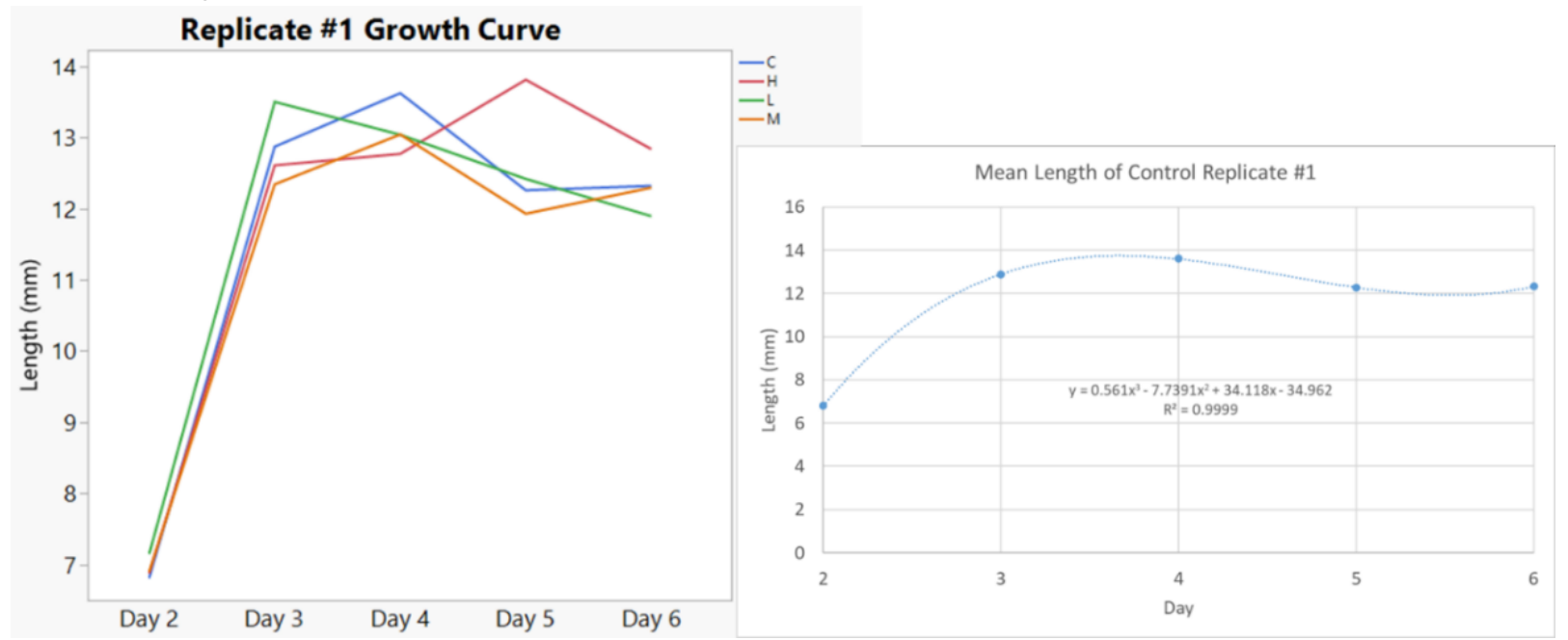

Figure 4.12: Growth curves for the four treatments for replicate \#1. 
Plotted in Figure 4.12 is the mean larval length against time for the insects collected from the four treatments in replicate \#1. The control (blue) and medium (orange) treatments reached peak mean lengths in the collection at day 4 . The low (green) treatment reached the peak mean length on the day 3 collection and the high (red) treatment group reached the peak mean length on day 5. The largest difference in calculated age to actual age was in the high treatment group at day 6 with a -1.4 day difference. At and after the day 4 collection, the high treatment group had a -1.0 day difference or greater indicating the estimated age of at least a day sooner than the actual age (Table 4.12). This is corroborated by the peak length for the high treatment occurring a day later than the control group. The low treatment age was artificially increased in the early collection days but decreased in the later collection days. This is corroborated by the peak of the length for the low treatment group occurring a day before the control group, an indication that the insects are progressing faster. The medium treatment group reached its peak length on the same day as the control but the had a -0.9 day difference for the day 4 collection and a -1.0 day difference on collection day 6. For the medium and high treatment groups, the fentanyl concentrations created an age estimation of 1.0 to 1.4 days shorter than the actual age in replicate \#1.

\subsection{Replicate \#2}

\section{Replicate \#2 Growth Curve}

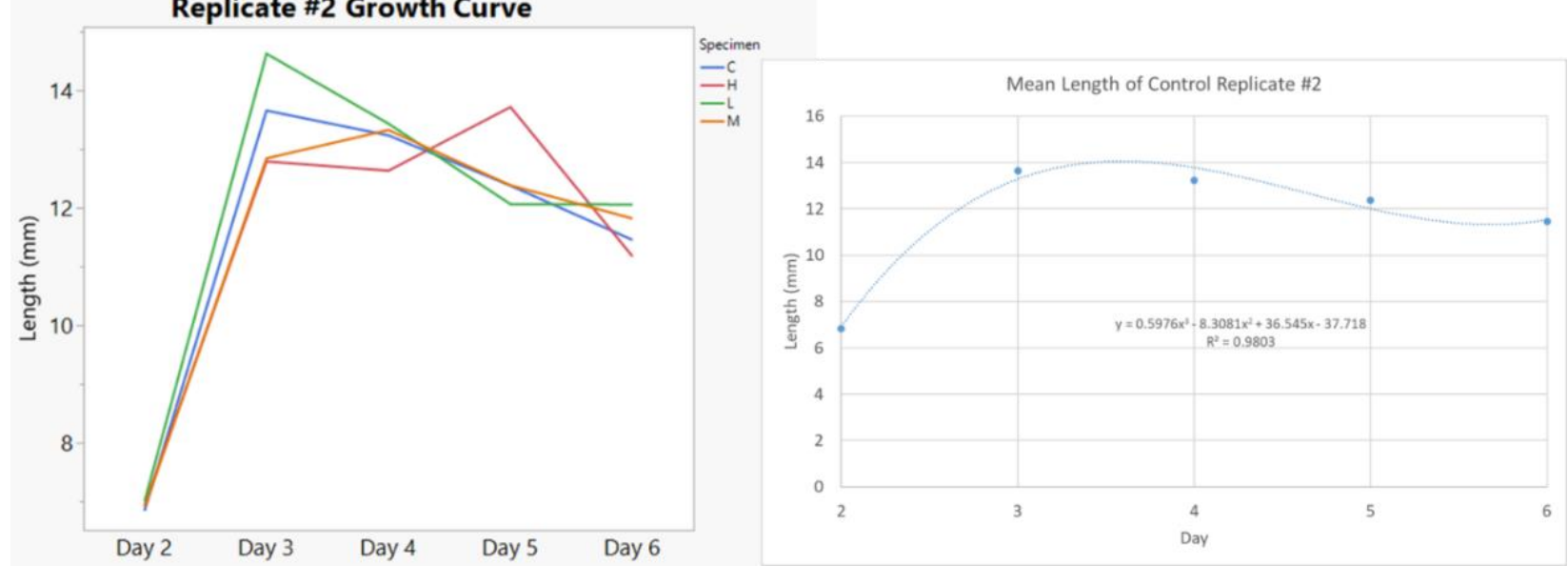

Figure 4.13: Growth curves for the four treatments for replicate \#2. 
Plotted in Figure 4.13 is the mean larval length against time for the insects collected from the four treatments in replicate \#2. The control (blue) and low (green) treatments reached peak mean lengths in the collection at day 3 . The medium (orange) treatment group reached its length peak one day later on collection day 4 and the high (red) treatment group reached its peak length two days later on collection day 5. The mean lengths for the control group for days 2 to 6 were plotted and a polynomial regression was applied (Figure 4.13). The largest difference in calculated age to actual age was in the high treatment group at day 4 with a -1.2 day difference. This is corroborated by the peak length for the high treatment occurring two days later than the control group. The low treatment age was artificially increased in the early collection days ( $0.6 \mathrm{~d}$ ) but decreased in the later collection days with a max of -1.0 day on collection day 6. The low treatment peaked at on the same day as the control group but was significantly longer than the control group (Figure 4.13). The medium had a -1.0 day difference for the day 4 collection and a -0.9 day difference on collection day 6 (Table 4.12). This is corroborated by the peak length for the medium treatment occurring one day later than the control group. For the medium and high treatment groups, the fentanyl concentrations created an age estimation of 0.9 to 1.2 days shorter than the actual age in replicate \#2.

\subsection{Replicate \#3}

Plotted in Figure 4.14 is the mean larval length against time for the insects collected from the five treatments in replicate \#3. The control (blue), medium (orange), and high (red) treatments reached peak mean lengths in the collection at day 4. Both the medium and high treatment groups peaked at a significantly longer length than the control group (Figure 4.14). The low (green) treatment group reached its peak length on day 5 . 


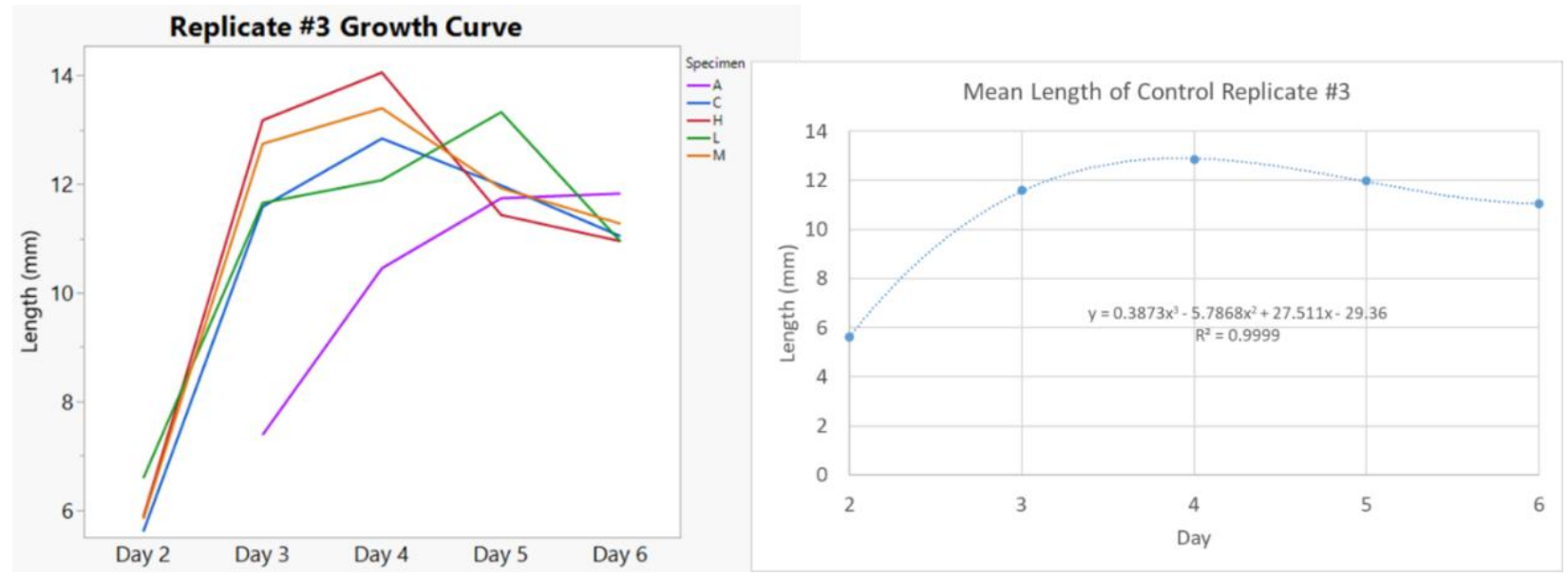

Figure 4.14: Growth curves for the five treatments for replicate \#3.

The authentic (purple) group appeared to peak on day 6 . The authentic set experienced the most difference in estimated age to actual age on day 6 at $-2.9 \mathrm{~d}$. The authentic set had a difference of more than -0.8 day for collection days 3 to 6 . This difference in age is corroborated in the peak length for the authentic set on day 6 or two days later than the control group. The low treatment experienced its greatest difference in estimated age to actual age at the day 5 collection of $-1.1 \mathrm{~d}$. The length for the medium and high treatment groups was elevated compared to control group with correlated to an increase in estimated age. The high reached a peak difference in estimated age at $0.9 \mathrm{~d}$ for the insects collected on day 3 and had a positive difference on days 4 and 5 . The medium reached a peak difference in estimated age at $0.6 \mathrm{~d}$ on collection day 3 but went down to an estimated $-0.4 \mathrm{~d}$ on collection day 6 (Table 4.12). The high and medium treatments experienced an increase in estimated age in the earlier collection days with a decrease as time continued to day 6 . The low and authentic groups experience a decrease in estimated age from the early collection days and persisted through the collection days.

\subsection{Total}

Plotted in Figure 4.15 is the mean larval length against time for the insects collected from the five treatments in all three replicates combined. The control (blue) and medium (orange) treatments reached peak mean lengths in the collection at day 4. The low (green) treatment reached the peak mean length 
on the day 3 collection and the high (red) treatment group reached the peak mean length between day 4 and day 5 .

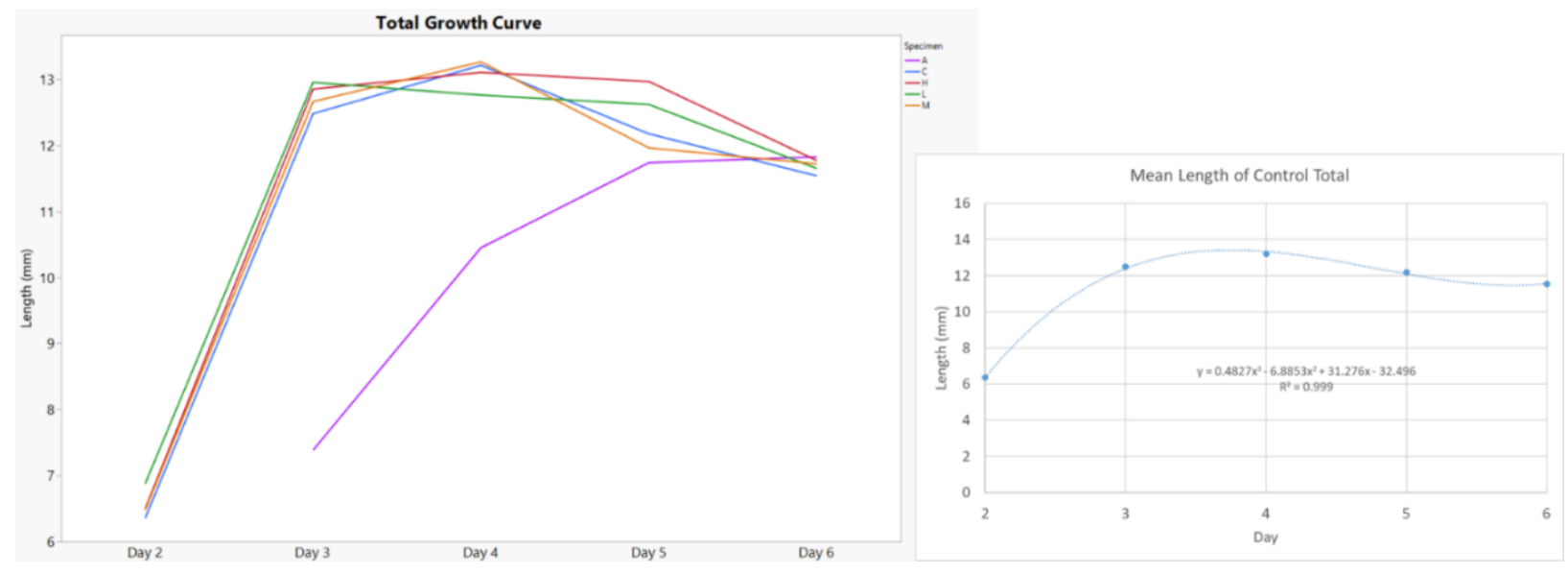

Figure 4.15: Growth curves for the five treatments for all three replicates combined.

The authentic (purple) group appeared to peak on day 6. For the combined means for the low, medium, and high treatment groups, there was a slight increase in age estimation on collection days 3 and 4 but the age estimation decreased for collection days 5 and 6 . There was a trend in all three replicates of an increase or no change in age on collection day 3 followed by a steady decrease in the later collection days. For the authentic set, the difference in the estimated age to actual at collection day 3 was $-0.9 \mathrm{~d}$ and steadily decreased down to $-3.2 \mathrm{~d}$ by collection day 6 . The estimated age for the treatments was lowest on collection day 6 was the lowest in each treatment group ranging from -0.6 to $-3.2 \mathrm{~d}$.

The maximum lengths of the larva were also compared for each treatment and replicate (Figure 4.16). The control groups reach their maximum on collection day 4 for all the replicates but the treatment groups lacked a true pattern. The authentic or validation set in replicate \#3 only reached its maximum length at day 6 , assuming that it did not increase in length as day 6 was the last day collected as larva. The low treatment group obtained the most variation with peak maximum lengths at collection day 3 , day 4 , and day 5 for replicates $\# 2, \# 1$, and $\# 3$ respectively. The control group growth curves maintained a distinct pattern, while the treatment groups did not maintain this pattern. The control length increased until the 
peak length, followed by a steady and steep decrease as the insects prepared for pupation. The high treatment observed the most variation in this patter with typically slower time to reach a maximum length and then a slower decrease in length.

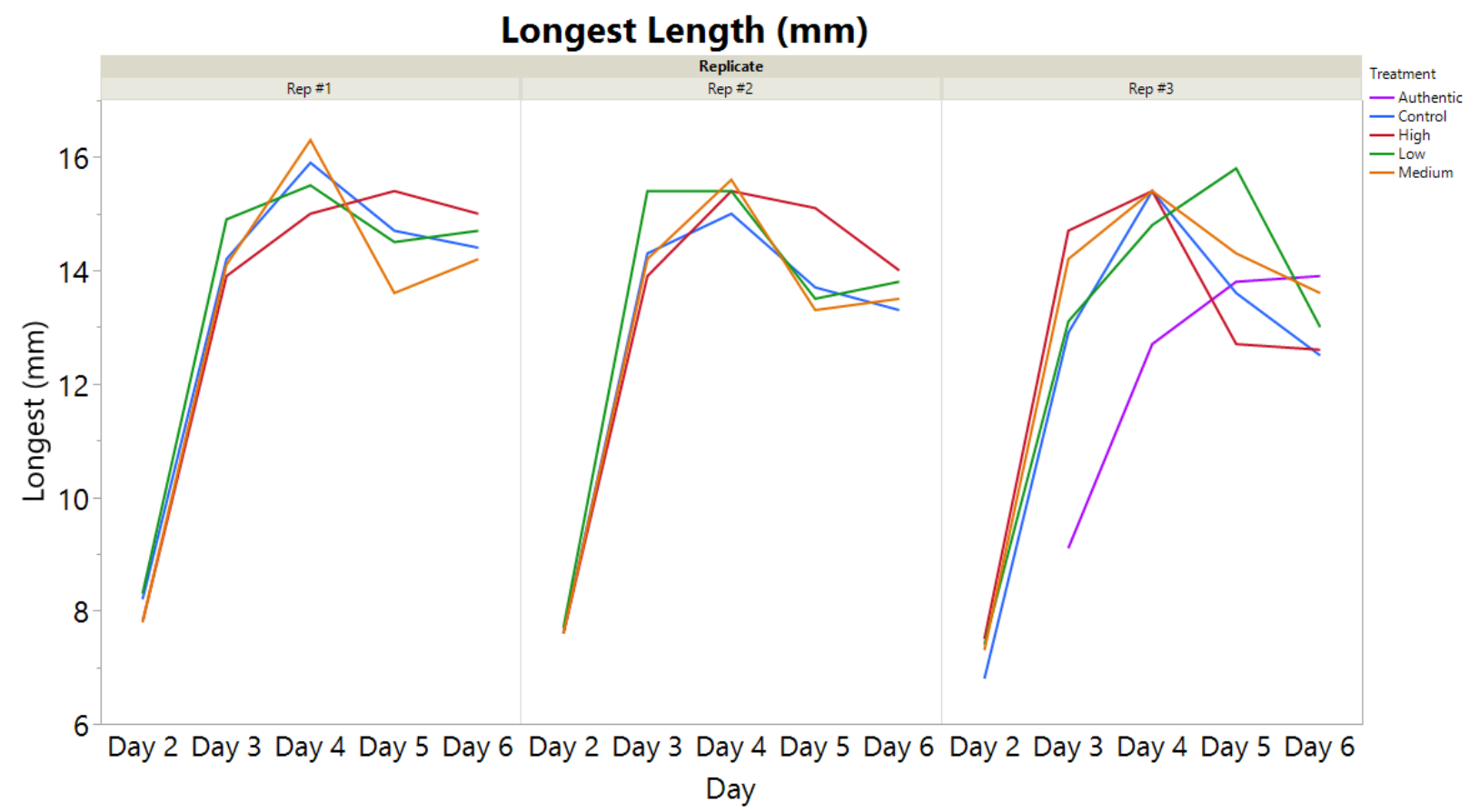

Figure 4.16: The maximum length measured from the larva collected on each day.

Table 4.11: Equations for the growth curve of the control group for each replicate and as a total.

\begin{tabular}{ccc}
\hline Control Growth Curve & Simulation equation & $\mathrm{R}^{2}$ \\
\hline Replicate \#1 & $\mathrm{L}=0.561 \mathrm{x}^{3}-7.7391 \mathrm{x}^{2}+34.118 \mathrm{x}-34.962$ & 0.999 \\
Replicate \#2 & $\mathrm{L}=0.5976 \mathrm{x}^{3}-8.3081 \mathrm{x}^{2}+36.545 \mathrm{x}-37.718$ & 0.980 \\
Replicate \#3 & $\mathrm{L}=0.7863 \mathrm{x}^{3}-10.672 \mathrm{x}^{2}+45.976 \mathrm{x}-49.729$ & 0.995 \\
Total & $\mathrm{L}=0.4827 \mathrm{x}^{3}-6.8853 \mathrm{x}^{2}+31.276 \mathrm{x}-32.496$ & 0.999 \\
\hline
\end{tabular}


Table 4. 12: Age estimations generated using the equations from Table 4.11. Estimated ages were compared to the actual day of collection to calculate a difference $(\Delta)$ age $(d)$.

\begin{tabular}{|c|c|c|c|c|c|c|c|c|c|c|}
\hline \multirow{3}{*}{ Treatment } & \multicolumn{10}{|c|}{ Replicate \#1 } \\
\hline & \multicolumn{5}{|c|}{ Estimated Age (d) } & \multicolumn{5}{|c|}{$\Delta$ Age $(\mathrm{d})$} \\
\hline & Day 2 & Day 3 & Day 4 & Day 5 & Day 6 & Day 2 & Day 3 & Day 4 & Day 5 & Day 6 \\
\hline Control & 2.0 & 3.0 & 4.0 & 5.0 & 6.0 & 0.0 & -0.0 & -0.0 & 0.0 & 0.0 \\
\hline Low & 2.0 & 3.3 & 4.5 & 4.9 & 5.5 & 0.0 & 0.3 & 0.5 & -0.1 & -0.5 \\
\hline Medium & 2.0 & 2.8 & 3.1 & 5.4 & 5.0 & 0.0 & -0.2 & -0.9 & 0.4 & -1.0 \\
\hline High & 2.0 & 2.9 & 3.0 & 3.7 & 4.6 & 0.0 & -0.1 & -1.0 & -1.3 & -1.4 \\
\hline
\end{tabular}

Replicate \#2

\begin{tabular}{|c|c|c|c|c|c|c|c|c|c|c|}
\hline \multirow{2}{*}{ Treatment } & \multicolumn{5}{|c|}{ Estimated Age (d) } & \multicolumn{5}{|c|}{$\Delta$ Age $(\mathrm{d})$} \\
\hline & Day 2 & Day 3 & Day 4 & Day 5 & Day 6 & Day 2 & Day 3 & Day 4 & Day 5 & Day 6 \\
\hline Control & 2.0 & 3.2 & 4.4 & 4.8 & 5.9 & -0.0 & 0.2 & 0.4 & -0.2 & -0.1 \\
\hline Low & 2.0 & 3.6 & 4.2 & 5.0 & 5.0 & 0.0 & 0.6 & 0.2 & -0.0 & -1.0 \\
\hline Medium & 2.0 & 2.9 & 3.0 & 4.8 & 5.1 & 0.0 & -0.1 & -1.0 & -0.2 & -0.9 \\
\hline High & 2.0 & 2.8 & 2.8 & 4.1 & 5.7 & 0.0 & -0.2 & -1.2 & -0.9 & -0.3 \\
\hline
\end{tabular}

Replicate \#3

\begin{tabular}{|c|c|c|c|c|c|c|c|c|c|c|}
\hline \multirow{2}{*}{ Treatment } & \multicolumn{5}{|c|}{ Estimated Age (d) } & \multicolumn{5}{|c|}{$\Delta$ Age $(\mathrm{d})$} \\
\hline & Day 2 & Day 3 & Day 4 & Day 5 & Day 6 & Day 2 & Day 3 & Day 4 & Day 5 & Day 6 \\
\hline Control & 2.0 & 3.0 & 4.1 & 5.0 & 6.1 & 0.0 & 0.0 & 0.1 & -0.0 & 0.1 \\
\hline Low & 2.1 & 3.0 & 3.2 & 3.9 & 6.0 & 0.1 & 0.0 & -0.8 & -1.1 & 0.0 \\
\hline Medium & 2.0 & 3.6 & 3.9 & 5.0 & 5.6 & 0.0 & 0.6 & -0.1 & 0.0 & -0.4 \\
\hline High & 2.0 & 3.9 & 3.9 & 5.4 & 6.8 & 0.0 & 0.9 & -0.1 & 0.4 & 0.8 \\
\hline Authentic & - & 2.2 & 2.7 & 3.1 & 3.1 & - & -0.8 & -1.3 & -1.9 & -2.9 \\
\hline
\end{tabular}

\section{Total}

\begin{tabular}{|c|c|c|c|c|c|c|c|c|c|c|}
\hline \multirow{2}{*}{ Treatment } & \multicolumn{5}{|c|}{ Estimated Age (d) } & \multicolumn{5}{|c|}{$\Delta$ Age (d) } \\
\hline & Day 2 & Day 3 & Day 4 & Day 5 & Day 6 & Day 2 & Day 3 & Day 4 & Day 5 & Day 6 \\
\hline Control & 2.0 & 3.0 & 4.2 & 4.9 & 6.0 & 0.0 & 0.0 & 0.2 & -0.1 & -0.0 \\
\hline Low & 2.1 & 3.2 & 4.5 & 4.6 & 5.4 & 0.1 & 0.2 & 0.5 & -0.4 & -0.6 \\
\hline Medium & 2.0 & 3.1 & 4.1 & 5.1 & 5.3 & 0.0 & 0.1 & 0.1 & 0.1 & -0.7 \\
\hline High & 2.0 & 3.2 & 4.3 & 4.4 & 5.3 & 0.0 & 0.2 & 0.3 & -0.6 & -0.8 \\
\hline Authentic & - & 2.1 & 2.5 & 2.8 & 2.8 & - & -0.9 & -1.5 & -2.2 & -3.2 \\
\hline
\end{tabular}




\subsubsection{Discussion}

This study presents morphometric measures of length, width, and mass for the insects collected; however, larval length was the most significant physical character. In comparison of individual collection days, at least one of the treatment groups (low, medium, or high) was significantly different from the control group over all three replicates. In all three replicates, the maximum larval length reached was higher in the high treatment group compared to the control group. In replicates \#2 and \#3, the low treatment group reached a maximum length greater than that of the control group and in replicate \#1 the maximum lengths were not significantly different between the two groups. The medium treatment group achieved a maximum length greater than the control group in replicate \#3, a similar max length in replicate \#2, and a lower maximum length in replicate \#1. Using a polynomial equation on the growth curve of the control group, the estimation of age of the insects showed that there was often an increase in growth at the early life stages, followed by a decrease in the later larval stages. The estimated age was compared to the day collected for the age of the insect. A positive difference indicates an increase in estimated age compared to the actual age and a negative number indicates a decrease in estimated age compared to the actual age. A positive number also indicates a faster development rate, and a negative number indicates a delay in development. In the low treatment group across all three replicates, the insects collected on day 3 had a neutral or increase in age compared to the actual age and by day 5 or 6 had a decrease in age estimation compared to the actual age. The largest discrepancy in actual age to estimated age for the low treatment group was -1.1 in the insects collected on day 5 in replicate \#3. For the medium group, the age estimation starting at collection day 3 for replicates \#1 and \#2 was decreased compared to the actual age and steadily decreased until collection day 6 with a final decrease of $-1.0 \mathrm{~d}$ age in both replicates. For replicate \#3, the medium treatment group had an increase estimation of $0.6 \mathrm{~d}$ at collection day 3 and then decreased to a final estimation of $-0.4 d$ at collection day 6 . For replicates \#1 and \#2, the high treatment consistently had a decreased estimation of age compared to the actual age of the insect beginning on collection day 3 . For replicate \#3, the high had an increased estimation for age for most of 
the collection days compared to the actual age, this could be due to the extreme elevation of the length of the high treatment group compared to the control. The high was significantly higher for the max length on day 4 and obtained peak length at the same time as the control group which could have led to the increased estimation. The authentic set was only applied to replicate \#3 and the age estimation was always decreased compared to the actual age beginning at collection day 3 and continuing through collection day 6 . The authentic set experienced the greatest discrepancy from estimated to actual age ranging from $-0.8 d$ at collection day 3 to $-2.9 d$ on collection day 6 . The peak maximum length for the authentic set was finally reached at collection day 6 , a full 2 days after the peak length was achieved for the control group. A reason for the difference in the authentic set compared to the medium and high treatments could be the presence of metabolites norfentanyl and 4-ANPP in the authentic liver specimen. The treatment livers were not fortified with any metabolites and none were detected in the liver extracted from collection days. The metabolite 4-ANPP is a known active metabolite in human metabolism pathways, and it is unknown how this compound will impact the insects. For the combined replicates, the average mean for the low, medium, and high treatment estimated ages were elevated compared to the actual age at collection day 3 but decreased as time elapsed and ranged from -0.6 to $-0.8 d$ by collection day 6 . For the authentic set, the difference in the estimated age at collection day 3 was $-0.9 d$ and decreased to $-3.2 \mathrm{~d}$ by collection day 6 . This was the largest discrepancy and an estimation of minPMI on the larval length collected on day 6 would be underestimated by 3 days.

To estimate the ${ }_{\min } \mathrm{PMI}$, the age of the oldest insects collected need to be estimated by identification of the insect life stage or by estimation based on larval length $(2,17)$. Drugs or toxins can impact the growth and development of insects which in turn can impact the $\min P M I$ estimations $(39,50$, 157). Even though there have not been publications for fentanyl effects on the growth of necrophagous insects, other opioids have been investigated $(39,157)$. El-Samad et al. investigated the effects of tramadol on L. sericata where they observed an increase in the time for the insects to progress from egg 
to adult eclosion (45). The control group in their study reached adulthood after 11.7 days but the three treatment groups ranged from 13.9 to 15.1 days to reach eclosion (45). They also observed an increase in larval length for the treatment groups with the maximum length achieved faster than the control group indicative of an accelerated larval growth but the treatment groups still took longer to emerge in the later stages (45). A similar trend was observed in the low treatment in this study and in the overall combination of the replicates where the treatment groups grew longer than the control, reached the maximum length faster but then still slowed as they approached the day 6 collection. Gosselin et al. explored the impact that methadone has on L. sericata over five treatment groups (44). The study did not observe any significant impact of the drug on the growth on the insect however, only empty puparium and adult eclosion was measured for the impact of development (44). The effects of heroin on Boettcherisca peregrina was investigated by Goff et al. by dosing live rabbits for four treatment concentrations plus a control (158). They also observed a longer max length in the treatment groups and a faster maximum length (158). The treatment groups also reached pupation before the control group but the control group was the first to emerge with all four treatments taking longer to emerge as adults (158). This is also similar to our study where we observed a longer maximum length and in the lower treatment reaching maximum length faster than the control group but slowing down in development as development progressed. The effect of morphine on various blow fly species has been evaluated $(47,159,160)$. George et al. investigated the effect of morphine on Calliphora stygia but they did not observe any significant effects of the drug on the growth of the insect across four treatments that included a control (47). A potential limitation of their study was only four time segments: day 4 (feeding), day 7 (wandering), pupation, and adult eclosion were evaluated (47). Bourel et al. dosed rabbits with lethal concentrations of morphine to evaluate the effects of the drug on L. sericata and discovered the insects developed at a slower rate (159). They estimated the error estimation in $\min _{\mathrm{PMI}} \mathrm{Po}$ be as great as $24 \mathrm{~h}$ in the larval estimations and that estimations based on puparia could be erroneous if the drugs is not taken into account (159). Rassi et al. 
investigated the effect of morphine on Chrysomya albiceps and calculated an underestimation of the ${ }_{\min } \mathrm{PMI}$ by $72 \mathrm{~h}$ for the larval development and 24 to $48 \mathrm{~h}$ based on the puparial development (160). Like the study presented here, they found an increase in the maximum larval length in the higher treatment groups compared to the control (160).

\subsubsection{Conclusion}

The low, medium, and high treatment groups reached a greater maximum length compared to the control group in most of the replicates in terms of maximum mean length between collection days 3 and 6. The authentic set experienced the greatest decrease in estimated age $(-2.9 \mathrm{~d})$ compared to actual age in replicate \#3. This is an underestimation of $2.9 \mathrm{~d}$ for the age of the larva collected at day 6 in replicate \#3. The largest discrepancy in age estimation for the high treatment group was $-1.4 \mathrm{~d}$ on collection day 6 in replicate \#1, which is an underestimation of $1.4 \mathrm{~d}$. The high treatment also had large underestimations of $-1.3 d,-1.2 d$, and $-1.0 d$ at collection day 5 replicate \#1, collection day 4 replicate \#2, and collection day 4 replicate \#1, respectively. The low and medium treatment groups also had at least a $-1.0 \mathrm{~d}$ underestimation of age at two or more collection days. Every treatment group had an underestimation in age of $1 \mathrm{~d}$ in at least one day of collection among the three replicates. With the impact of fentanyl on the length of the larval length, it can be concluded that fentanyl can reduce the larval growth by at least 1 day and up to 3 days. Even though a significant trend was not identified across the replicates for all treatments, the impact on age estimation based on length and the variability of the length in the insects collected from the treatment groups indicates that caution must be used when estimating $\min ^{\mathrm{PMI}}$ when fentanyl is present in the corpse. 


\subsection{Section 3: Quantitation of Fentanyl and Metabolites from Larva and Pupa (Lucilia sericata)}

\subsubsection{Overview}

This section presents the toxicological findings for the larva and pupa for task 1.e. The validated QuEChERS extraction presented in section 1 of this chapter was used for extraction of the feeding media liver and the insect tissue. Presented is the survey of fentanyl and metabolites detected in the larva and pupa. For task 3.a, a correlation plot was created for a comparison of the fentanyl concentrations detected in the insect tissue to the liver fentanyl concentrations to establish any correlation of the concentrations. The concentrations detected for the set of insects that fed on the authentic liver source were compared to theoretical concentrations estimated from the correlation plots. The goal of this section is to evaluate the toxicological value of the insect life stages, quantify any metabolites detected for evidence of metabolism by the insect, and to draw any conclusions of the liver tissue concentration to insect tissue concentrations.

\subsubsection{Toxicology Matrix and Correlations}

Detection of drugs in pupa and empty puparium has a benefit over larva because they persist on the remains longer and are potentially available after other suitable toxicology specimens are unavailable (111). Wood et al. detected nordiazepam and its metabolite oxazepam in larva and pupa that resulted from rearing on beef heart tissue containing $1 \mathrm{ug} / \mathrm{g}(1 \mathrm{ppm})$ nordiazepam (161). Pien et al. performed a similar study with nordiazepam, analyzing larva, pupa, and empty puparium for nordiazepam and oxazepam in all three of the life stages of Calliphora vicina (162). When feeding on tissue with drugs present, the drugs are deposited in fat bodies of larva and the chitinized exoskeleton of the pupa (154). The function of the hardened, chitin cuticle of the pupa is to protect the insect from environment during metamorphosis (44). The structure is resistant to chemical attack, microorganisms, and weathering (44). Due to the structure of chitin, the drugs are often locked in and remain for an extended period of time (34). When pupa emerge to adult flies, the empty puparia remains behind with the drugs trapped in the chitin (44). Pupa and empty puparia, due to the strength of the chitin protected exoskeleton, can persist 
in the remains long after the soft tissue of the corpse have disappeared (34). This allows for toxicological analysis to take place on the pupa and empty puparia even in advanced decomposition or skeletonized remains when traditional toxicology specimens like blood, urine and organ tissue are not available for testing. The use of empty puparium for toxicological analysis has utility due to their ability to exist for extend periods on remains, even after skeletonization of the corpse (154). Miller et al. were able to isolate amitriptyline and nortriptyline from empty fly puparia and beetle exuvia recovered from mummified human remains in an indoor decomposition case (163). From stomach contents and analysis of the desiccated brain, multiple drug intoxication, including amitriptyline, was identified as the cause of death (163). The ability of empty puparia to remain in soil surrounding the remains for hundreds of years, makes empty puparia a prime target specimen for toxicology analysis in cadavers discovered in the late stage of decomposition (163).

Gosselin et al. identified strategies for standardizing entomotoxicological experimental designs for research and interpretation of results with a key aim of extracting target compounds with a straightforward methodology while maintaining sensitivity and minimizing matrix effects (111). Emphasis is placed on the reduction of matrix effects like co-eluting compounds while maintaining acceptable extraction recoveries (111). Da Silva et al. examined 55 entomotoxicology papers published between 1980 and 2016 , finding $21 \%$ did not use any analytical methods to quantify the toxicant in the insects, $18 \%$ used high performance liquid chromatography (HPLC) or GC/MS and $11 \%$ used radioimmunoassay (RIA) (61). In the papers examined, $57 \%$ did not mention an extraction technique and the studies that did employed a solid-phase extraction in $31 \%$ and a liquid-liquid extraction in $7 \%$ of the experiments (61). There is a need to standardize an extraction and analysis technique in the field of entomotoxicology (61). This study addressed that need by utilizing a QuEChERS extraction protocol combined with a LC-MS/MS analysis method. 
Recent studies have demonstrated the possibility of correlating concentrations of drugs extracted from insects compared to concentrations of the feeding substrate, however interpretation of results has remained a controversial issue $(39,111)$. Estimation to the cause of death or relationships of blood or liver tissue concentrations to drug concentrations recovered from insects feeding on a corpse remain unachieved and are subject to debate $(39,111)$. Identifying this quantitative relationship is difficult due to complicated influencing factors including: drug tropism in the body, postmortem redistribution, and the extraction and detection efficiencies of the analytical techniques $(39,61,111)$. Sallawad et al. highlighted this need and lack of valuable research into the factors limiting the assessment of correlating insect drug concentrations to tissue drug concentrations with the conclusion that further research into entomotoxicology is necessary (42). The first step in achieving correlation between blood/tissue and insect concentrations is to control or reduce the influencing factors by identifying correlations from insect drug concentrations to single tissue type source drug concentrations. To address this critical need, liver tissue fortified with fentanyl was the feeding media for the insects in this study. Concentrations were compared to determine any correlation.

\subsubsection{Methods}

The samples from the design in methods 4.2.3 were used for the toxicology analysis of the collected insects. Insects from collection days 3, 6, 10, and 21 were selected for toxicology analysis after the imaging and morphometric characteristic measurements (Figure 4.5). The target groups were feeding larva (day 3), wandering larva (day6), pupa (day 10), and empty puparium/adults (day21). Insects from each treatment level and day of collection were randomly selected $(n=5)$ and extracted using the validated QuEChERS extraction. Larval specimens were also randomly selected into pools of four for analysis of pooled samples $(n=5)$. Insects were assigned numbers using the random number generator function in excel ( 0 to 70 ) with the lowest numbers assigned to single replicates and the highest numbers selected for the pooled samples. Liver samples were collected at the time of egg placement (day 0) and at the day of collection to establish the initial tissue concentrations and to evaluate any metabolism of the drugs by 
the feeding media. A survey of the persistence and prevalence of fentanyl and metabolites was performed followed by a correlation plot of liver concentration to insect concentration to evaluate any correlation.

\subsubsection{Results}

\subsubsection{Survey of Drugs}

The previously discussed validated QuEChERS extraction was applied to the larva and pupa collected on days 3,6 , and 10 for all four treatments over three replicates with one additional treatment set of an authentic postmortem liver specimen containing fentanyl. The insects were analyzed as single samples and pooled together $(n=4)$ for five replicates from each sample time.

For the single and pooled larva collected on day 3, fentanyl was detected and quantitated in all treatments across all three replicates (Table 4.12). A trend emerged, as the concentration of the fortified liver treatment increased, the concentration of fentanyl observed in the larval extractions also increased. For the larva collected from the low treatment, the average concentration across the three replicates was $1.2 \pm 0.5 \mu \mathrm{g} / \mathrm{kg}$ in the single specimens and $1.2 \pm 0.5 \mu \mathrm{g} / \mathrm{kg}$ in the pooled larval samples. From the medium treatment, the average concentration across the three replicates was $9.1 \pm 4.1 \mu \mathrm{g} / \mathrm{kg}$ in the single specimens and $8.5 \pm 2.0 \mu \mathrm{g} / \mathrm{kg}$ in the pooled larval samples. From the high treatment, the average concentration across the three replicates was $30.0 \pm 10.5 \mu \mathrm{g} / \mathrm{kg}$ in the single specimens and $29.3 \pm 9.3$ $\mu \mathrm{g} / \mathrm{kg}$ in the pooled larval samples. For all the fentanyl extractions, the pooled samples generated similar concentrations to the single specimens but experienced reduced standard deviations. In the control insect extractions, no fentanyl or metabolites were detected in any of the replicates. In extracts of the larva from the low, medium, and high treatment groups, metabolites 4-ANPP and $\beta$-hydroxyfentanyl were not detected in any of the replicates. The metabolite norfentanyl was quantitated in the single and pooled larva collected from the high treatment livers in all three of the replicates. Norfentanyl concentrations for the larval high treatment groups quantitated at $3.3 \pm 1.5 \mu \mathrm{g} / \mathrm{kg}$ for the single larvae extraction and $2.1 \pm$ 
$1.2 \mu \mathrm{g} / \mathrm{kg}$ for the pulled larva extracts. Norfentanyl was detected in the pooled larva extractions from the medium treatment group in all three replicates with an average of $0.8 \pm 0.3 \mu \mathrm{g} / \mathrm{kg}$.

For the single and pooled larva extracted from the authentic liver treatment, fentanyl, and metabolites norfentanyl and 4-ANPP were quantitated. The authentic postmortem liver specimen was quantitated with an average fentanyl concentration of $111 \mu \mathrm{g} / \mathrm{kg}$, norfentanyl concentration of $8.1 \mu \mathrm{g} / \mathrm{kg}$, and a 4-ANPP concentration of $5.8 \mu \mathrm{g} / \mathrm{kg}$ at the time of egg placement. Since the metabolites were present in the liver at the time of egg placement, the metabolites cannot be used as proof of metabolism by the insects. For the single and pooled larva extractions for the authentic set, the fentanyl concentrations were $10.6 \pm 4.1 \mu \mathrm{g} / \mathrm{kg}$ and $9.3 \pm 1.2 \mu \mathrm{g} / \mathrm{kg}$, respectively. The concentration for the authentic liver and the fortified liver for the medium treatment were in similar concentration and the fentanyl concentrations quantitated from the larva extractions were in similar concentration (Table 4.13). No metabolites were detected in the fortified liver tissue on the day of collection. 
Table 4.13: Larval drug concentrations from day three collections for all three replicates.

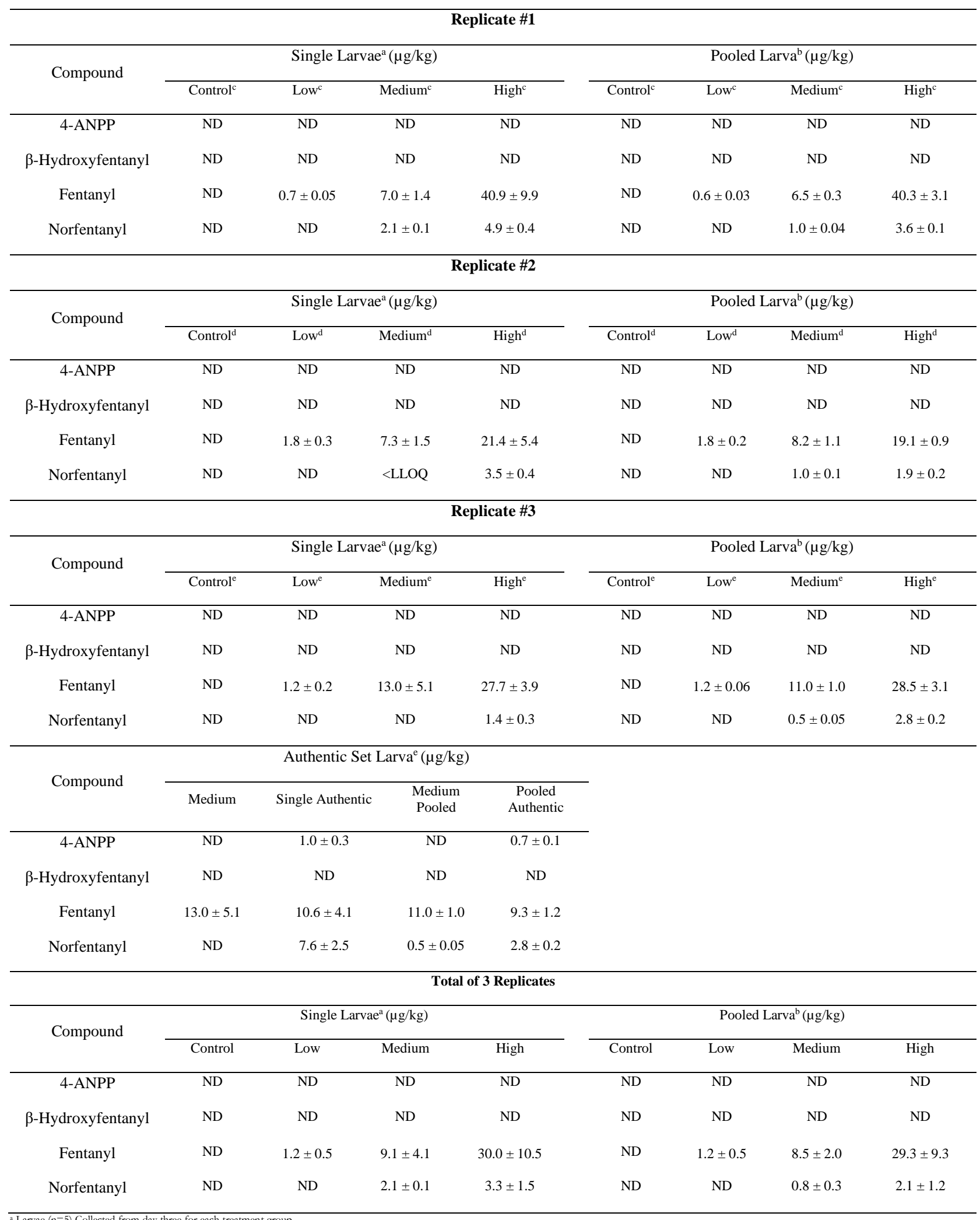

${ }^{a}$ Larvae ( $\left.\mathrm{n}=5\right)$ Collected from day three for each treatment group

${ }^{\mathrm{b}}$ Pooled Larva $(\mathrm{n}=5)$ Pooled specimen of 4 larva collected from day three for each treatment group

c Treatments: Control $(0 \mu \mathrm{g} / \mathrm{kg})$, Low $(6.0 \mathrm{\mu g} / \mathrm{kg})$, Medium $(23.3 \mu \mathrm{g} / \mathrm{kg})$, and High $(239 \mu \mathrm{gg} / \mathrm{kg})$ quantitated from liver tissue collected at time of egg placement

${ }^{d}$ Treatments: Control $(0 \mu \mathrm{g} / \mathrm{kg})$, Low $(12.6 \mathrm{\mu g} / \mathrm{kg})$, Medium $(53 \mathrm{\mu g} / \mathrm{kg})$, and High $(211 \mathrm{\mu g} / \mathrm{kg})$ quantitated from liver tissue collected at time of egg placement

c Treatments: Control $(0 \mu \mathrm{g} / \mathrm{kg})$, Low $(14.6 \mu \mathrm{g} / \mathrm{kg})$, Medium $(102 \mu \mathrm{g} / \mathrm{kg})$, and High $(209 \mu \mathrm{g} / \mathrm{kg})$ quantitated from liver tissue collected at time of egg placement 


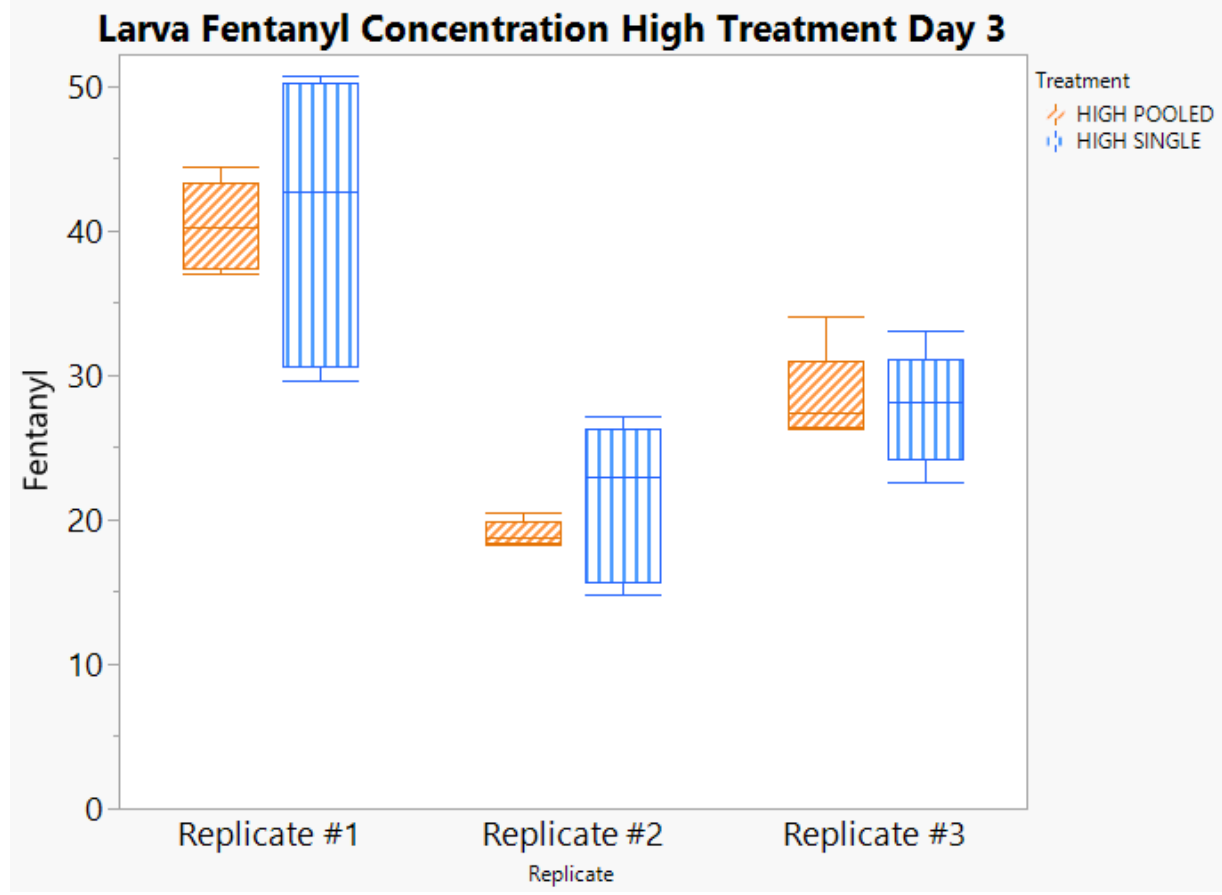

Figure 4.17: Box plot for the concentration of fentanyl quantitated for the single and pooled larva specimens collected from the high treatment liver on day 3 for all three replicates $(n=15)$.

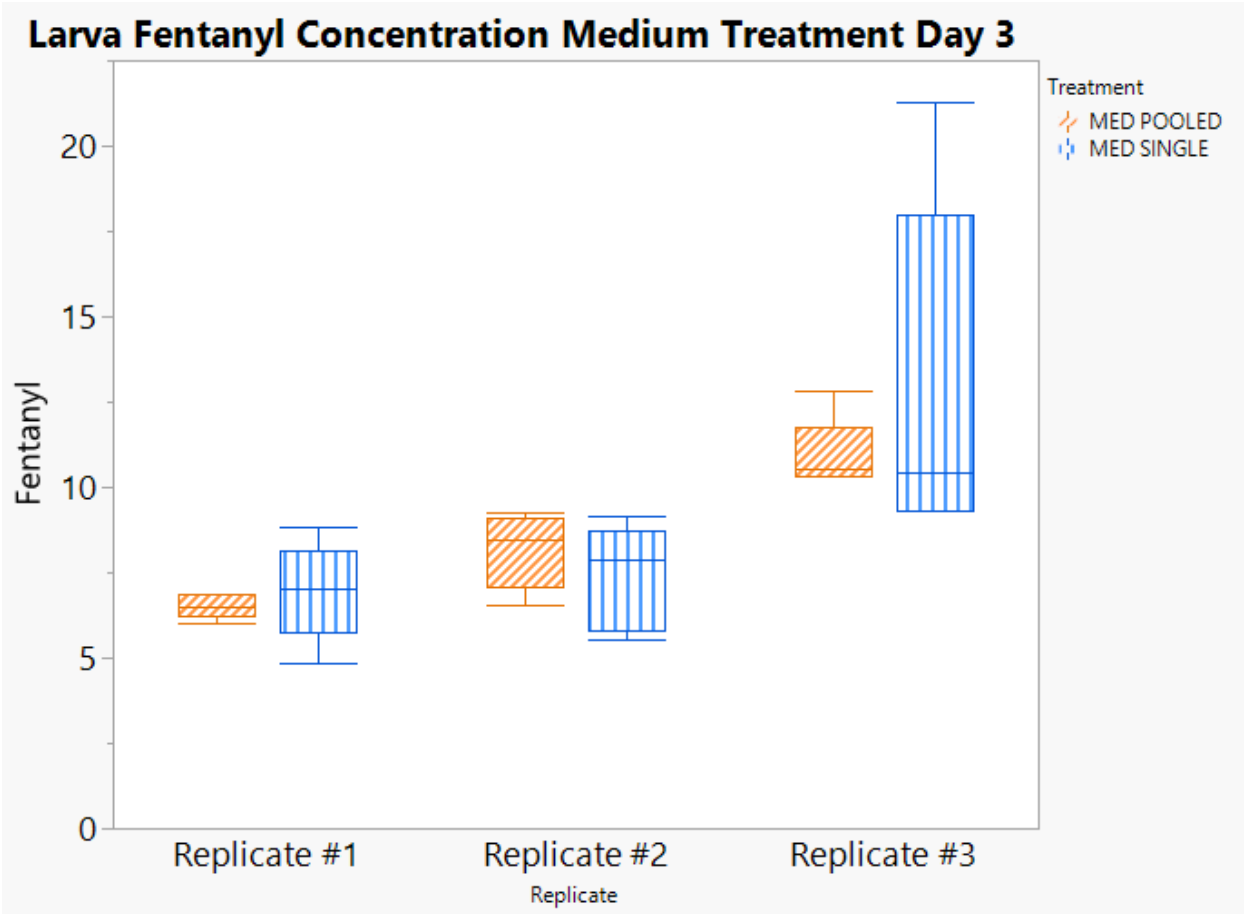

Figure 4.18: Box plot for the concentration of fentanyl quantitated for the single and pooled larva specimens collected from the medium treatment liver on day 3 for all three replicates $(n=15)$. 


\section{Larva Fentanyl Concentration Low Treatment Day 3}

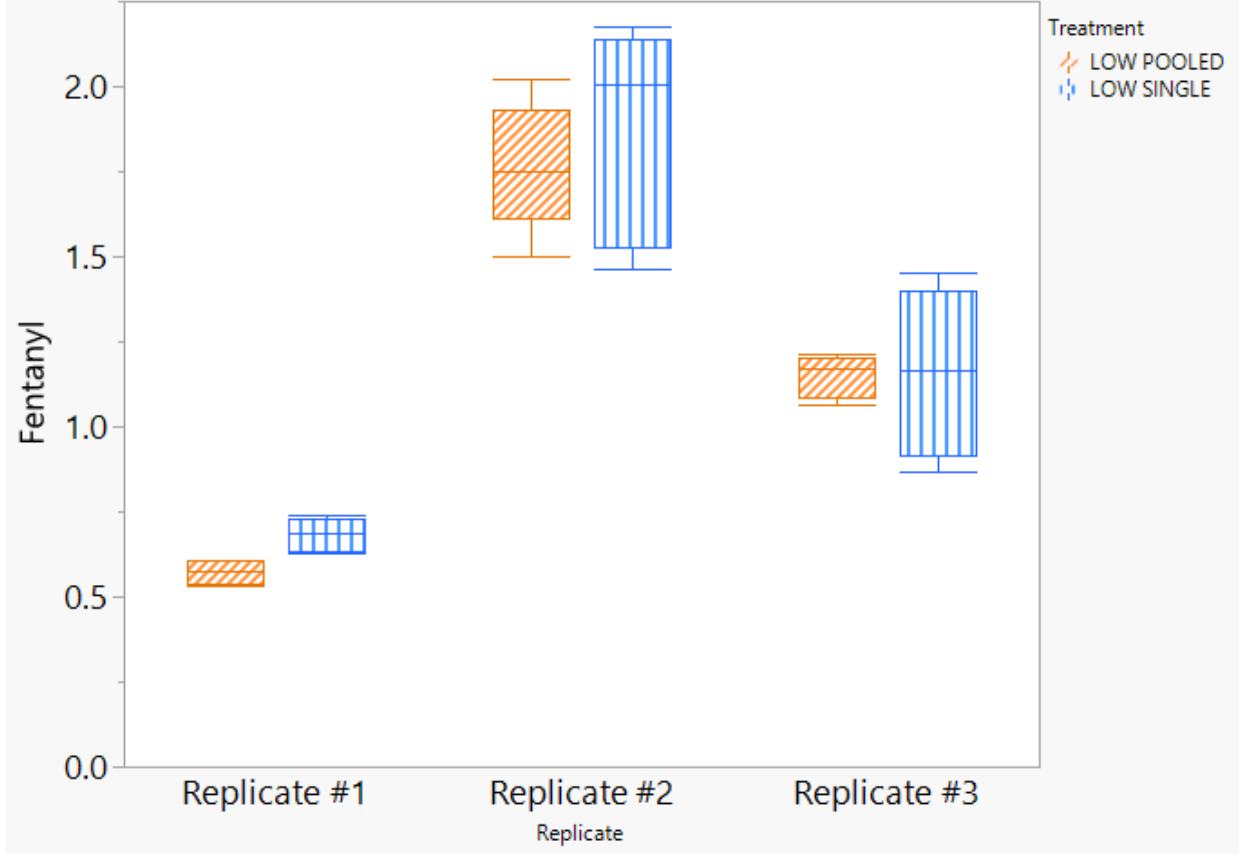

Figure 4.19: Box plot for the concentration of fentanyl quantitated for the single and pooled larva specimens collected from the medium treatment liver on day 3 for all three replicates $(n=15)$.

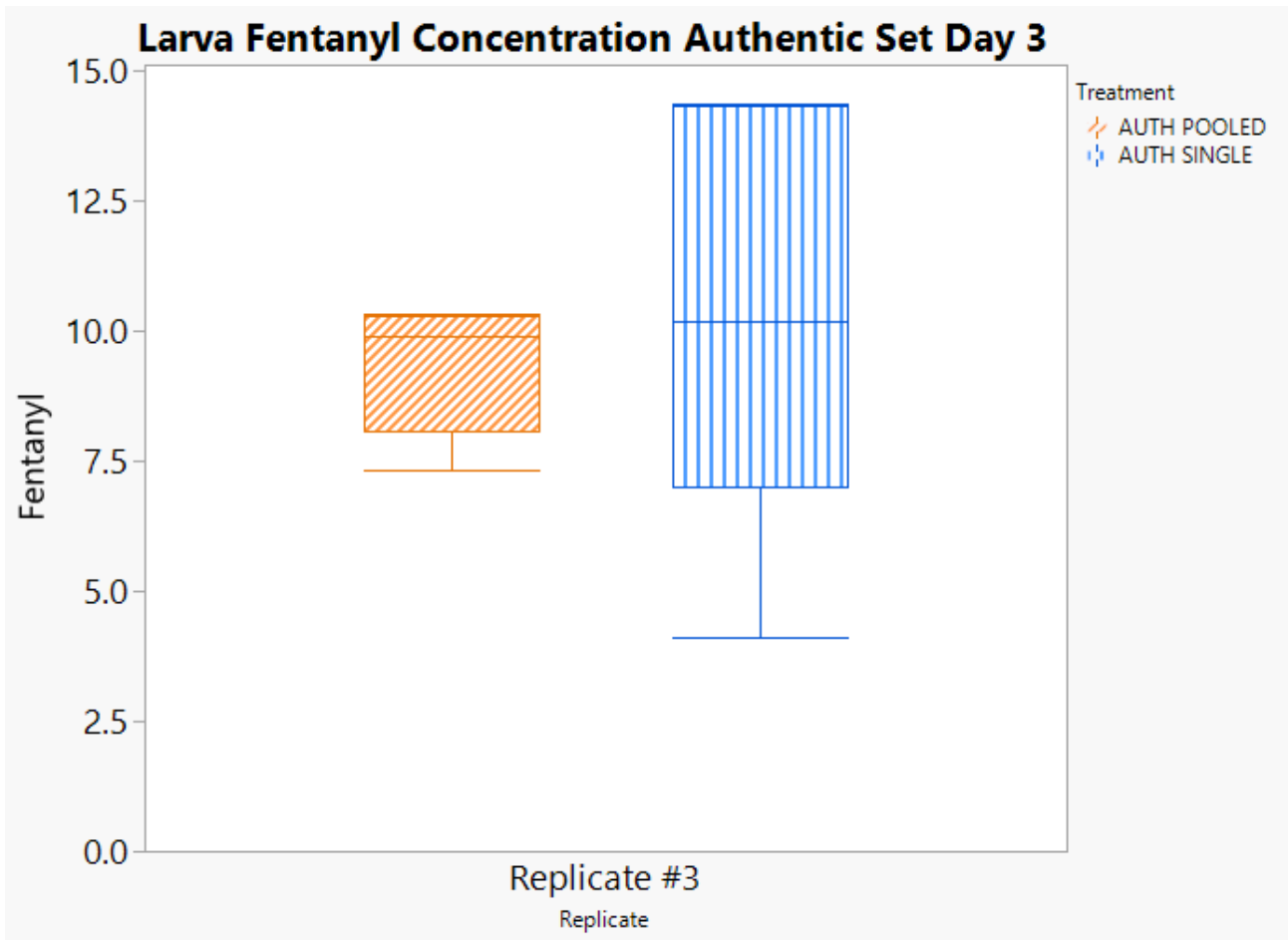

Figure 4.20: Box plot for the concentration of fentanyl quantitated for the single and pooled larva specimens collected from the low treatment liver on day 3 for all three replicates $(n=15)$. 


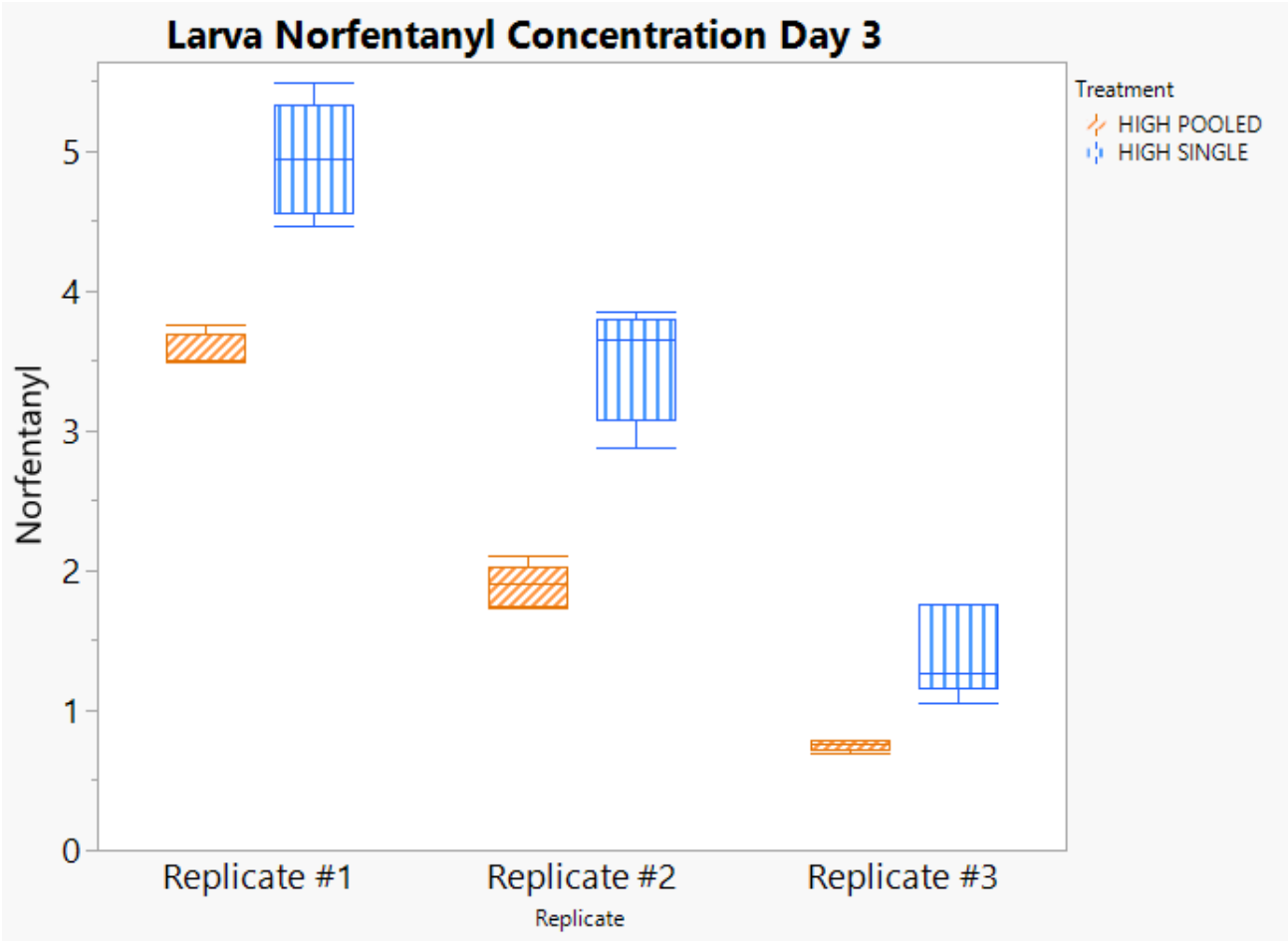

Figure 4.21: Box plot for the concentration of norfentanyl quantitated for the single and pooled larva specimens collected from the high treatment liver on day 3 for all three replicates $(n=15)$.

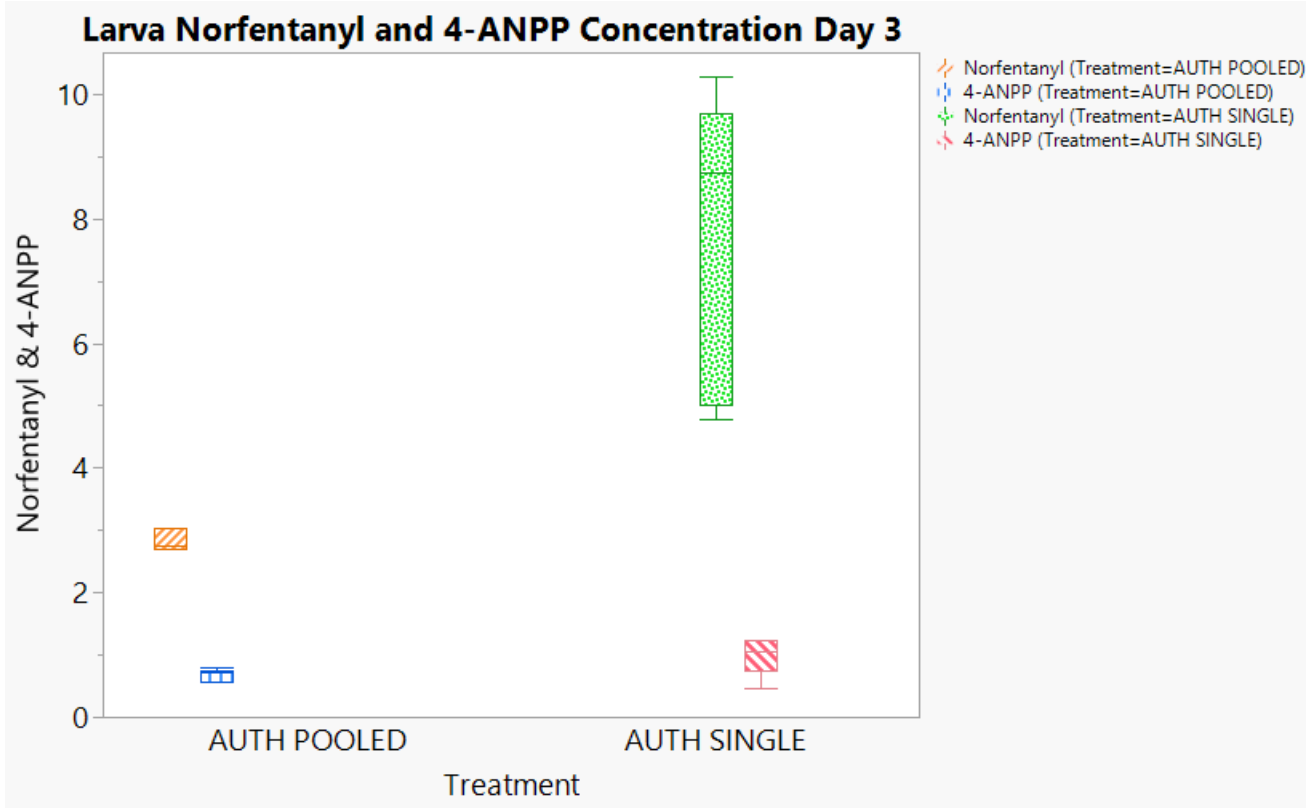

Figure 4.22: Box plot for the concentration of norfentanyl and 4-ANPP quantitated for the single and pooled larva specimens collected from the authentic treatment liver on day 3 for all three replicates $(n=5)$.

The box plot in figure 4.17 displays the concentration for fentanyl for the larva collected from the high treatment liver specimens across the three replicates. The variation for the pooled larval samples 
were less compared to the single specimens while the single specimens had a higher maximum and lower minimum than the pooled extractions. The box plot in figure 4.18 shows the concentration for fentanyl for the larva collected from the medium treatment liver specimens across the three replicates. The single extractions in replicate \#3 observed the greatest variation. The box plot in figure 4.19 exhibits the concentration for fentanyl for the larva collected from the low treatment liver specimens across the three replicates. The similar trend of the pooled extractions having less of a spread is observed for the low treatment groups as well. The box plot in figure 4.21 exhibits the concentration for norfentanyl for the larva collected from the high treatment liver specimens across the three replicates. The box plot in figure 4.22 displays the concentration for norfentanyl and 4-ANPP for the larva collected from the authentic postmortem liver specimen from replicate \#3.

The fentanyl and metabolite concentrations detected from the larva collected on day 6 was significantly lower than the concentrations detected on day 3 collections (Table 4.14). The highest mean detected from the larva in day 3 was $40.9 \mu \mathrm{g} / \mathrm{kg}$ compared to the highest detected fentanyl concentration of $0.9 \mu \mathrm{g} / \mathrm{kg}$ for the high treatment on day 6 . Fentanyl was quantitated in the high fortified liver pooled larva samples for all three replicates and was the only group for fentanyl to be detected in all three treatments. The metabolite 4-ANPP was not detected in any of the extractions, even in the authentic specimen that had 4-ANPP present when the eggs were placed. Norfentanyl was present in some of the extractions but the concentrations were reduced and was not detected as frequently. The metabolite $\beta$ hydroxyfentanyl was detected in multiple groups after not being observed in the day 3 collected specimens. $\beta$-hydroxyfentanyl was quantitated in the highest concentration at $0.9 \pm 0.3 \mu \mathrm{g} / \mathrm{kg}$ in the medium treatment pooled larval samples for replicate \#3. $\beta$-hydroxyfentanyl was also quantified in the pooled high treatment samples for replicates \#2 and \#3 while being detected below the LLOQ in single larval extractions for the medium and high treatments in replicate \#3. 
Table 4.14: Larval drug concentrations from day six collections for all three replicates.

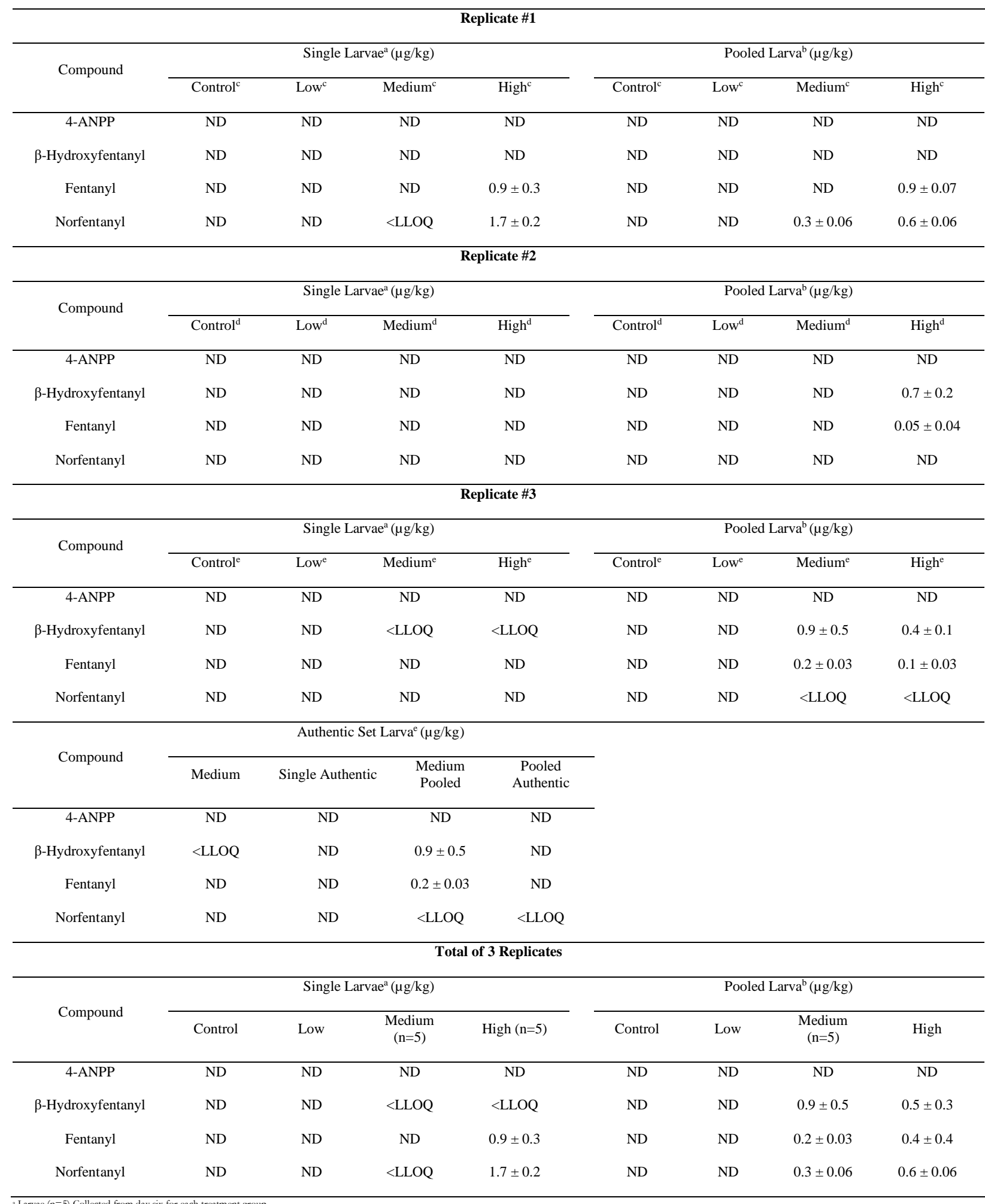

2 Larvae ( $\mathrm{n}=5)$ Collected from day six for each treatment group
b Pooled Larva ( $\mathrm{n}=5)$ Pooled specimen of 4 larva collected from day six for each treatment group

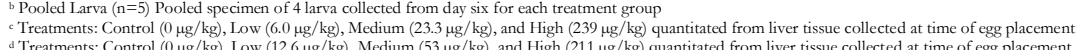

c Treatments: Control $(0 \mu \mathrm{g} / \mathrm{kg})$ L Low $(14.6 \mu \mathrm{g} / \mathrm{kg})$, Medium $(102 \mu \mathrm{g} / \mathrm{kg}$ ) and $\mathrm{High}(209 \mu \mathrm{\mu g} / \mathrm{kg})$ quantitated from liver tissue collected at time of egg placement 
The trend of decreased fentanyl concentrations continued to the pupal extractions (Table 4.15). Fentanyl was only detected in the high treatment pupal specimens and was not detected in all extractions. The single pupal extractions for all three replicates did not detect any fentanyl and fentanyl was only quantitated in the high pooled sample for replicate \#1 and below the LLOQ in replicates \#2 and \#3. Detection and quantitation of the metabolites was also less frequent. Norfentanyl was quantitated in all three treatments for both the single and the pooled pupa specimens in replicate \#1 but was only detected below the LLOQ for the single and pooled high specimens in replicate \#2. Norfentanyl was quantitated in the single and pooled pupal extractions for the high treatment group in replicate \#3. For the pupa collected from feeding on the authentic liver specimen, norfentanyl was quantitated in the single and pooled specimens while fentanyl was only detected below the LLOQ in the single and pooled extractions for fentanyl. $\beta$-hydroxyfentanyl was also detected in the pupal extractions indicating that this metabolite can persist from the larval stage to the pupal life stage. 
Table 4.15: Pupal drug concentrations from day 10 collections for all three replicates.

\begin{tabular}{|c|c|c|c|c|c|c|c|c|}
\hline \multicolumn{9}{|c|}{ Replicate \#1 } \\
\hline \multirow{2}{*}{ Compound } & \multicolumn{4}{|c|}{ Single Pupae ${ }^{\mathrm{a}}(\mu \mathrm{g} / \mathrm{kg})$} & \multicolumn{4}{|c|}{ Pooled Pupa ${ }^{\mathrm{b}}(\mu \mathrm{g} / \mathrm{kg})$} \\
\hline & Control $^{\mathrm{c}}$ & Low $^{\mathrm{c}}$ & Medium $^{c}$ & $\mathrm{High}^{\mathrm{c}}$ & Control $^{c}$ & Low $^{\mathrm{c}}$ & Medium $^{c}$ & $\mathrm{High}^{\mathrm{c}}$ \\
\hline 4-ANPP & ND & ND & ND & ND & ND & ND & ND & ND \\
\hline$\beta$-Hydroxyfentanyl & ND & ND & ND & $2.7 \pm 1.8$ & ND & ND & $1.1 \pm 0.2$ & $0.8 \pm 0.5$ \\
\hline Fentanyl & ND & ND & ND & ND & ND & ND & ND & $0.08 \pm 0.03$ \\
\hline Norfentanyl & ND & $2.0 \pm 0.4$ & $1.9 \pm 0.1$ & $1.8 \pm 0.3$ & ND & $0.5 \pm 0.1$ & $0.5 \pm 0.1$ & $0.5 \pm 0.04$ \\
\hline \multicolumn{9}{|c|}{ Replicate \#2 } \\
\hline \multirow{2}{*}{ Compound } & \multicolumn{4}{|c|}{ Single Pupae $(\mu \mathrm{g} / \mathrm{kg})$} & \multicolumn{4}{|c|}{ Pooled Pupa ${ }^{\mathrm{b}}(\mu \mathrm{g} / \mathrm{kg})$} \\
\hline & Control $^{d}$ & Low $^{d}$ & Medium $^{\mathrm{d}}$ & $\operatorname{High}^{\mathrm{d}}$ & Control $^{\mathrm{d}}$ & Low $^{d}$ & Medium $^{\mathrm{d}}$ & $\operatorname{High}^{\mathrm{d}}$ \\
\hline 4-ANPP & ND & ND & ND & ND & ND & ND & ND & ND \\
\hline$\beta$-Hydroxyfentanyl & ND & ND & ND & ND & ND & ND & ND & ND \\
\hline Fentanyl & ND & ND & ND & ND & ND & ND & ND & $<$ LLOQ \\
\hline Norfentanyl & ND & ND & ND & $<$ LLOQ & ND & ND & ND & $<$ LLOQ \\
\hline \multicolumn{9}{|c|}{ Replicate \#3 } \\
\hline \multirow{2}{*}{ Compound } & \multicolumn{4}{|c|}{ Single Pupae $(\mu \mathrm{g} / \mathrm{kg})$} & \multicolumn{4}{|c|}{ Pooled Pupa ${ }^{\mathrm{b}}(\mu \mathrm{g} / \mathrm{kg})$} \\
\hline & Control $^{\mathrm{e}}$ & Low $^{\mathrm{e}}$ & Medium $^{e}$ & $\mathrm{High}^{\mathrm{e}}$ & Control $^{\mathrm{e}}$ & Low $^{e}$ & Medium $^{\mathrm{e}}$ & $\mathrm{High}^{\mathrm{e}}$ \\
\hline 4-ANPP & ND & ND & ND & ND & ND & ND & ND & ND \\
\hline$\beta$-Hydroxyfentanyl & ND & ND & ND & ND & ND & ND & ND & ND \\
\hline Fentanyl & ND & ND & ND & ND & ND & ND & ND & $<$ LLOQ \\
\hline Norfentanyl & ND & ND & ND & $1.7 \pm 0.1$ & ND & ND & ND & $0.5 \pm 0.03$ \\
\hline \multirow[b]{2}{*}{ Compound } & \multicolumn{4}{|c|}{ Authentic Set $\operatorname{Pupa}^{\mathrm{e}}(\mu \mathrm{g} / \mathrm{kg})$} & & & & \\
\hline & Medium & Single Authentic & $\begin{array}{l}\text { Medium } \\
\text { Pooled }\end{array}$ & $\begin{array}{c}\text { Pooled } \\
\text { Authentic }\end{array}$ & & & & \\
\hline 4-ANPP & ND & ND & ND & ND & & & & \\
\hline$\beta$-Hydroxyfentanyl & ND & ND & ND & ND & & & & \\
\hline Fentanyl & ND & $<$ LLOQ & ND & $<$ LLOQ & & & & \\
\hline Norfentanyl & ND & $1.6 \pm 0.1$ & ND & $0.6 \pm 0.3$ & & & & \\
\hline
\end{tabular}

Total of 3 Replicates

\begin{tabular}{|c|c|c|c|c|c|c|c|c|}
\hline \multirow{2}{*}{ Compound } & \multicolumn{4}{|c|}{ Single Pupae $(\mu \mathrm{g} / \mathrm{kg})$} & \multicolumn{4}{|c|}{ Pooled Pupa ${ }^{\mathrm{b}}(\mu \mathrm{g} / \mathrm{kg})$} \\
\hline & Control & Low $(n=5)$ & $\begin{array}{l}\text { Medium } \\
(\mathrm{n}=5)\end{array}$ & High $(\mathrm{n}=5)^{*}$ & Control & Low & $\begin{array}{c}\text { Medium } \\
(\mathrm{n}=5)\end{array}$ & High \\
\hline 4-ANPP & ND & ND & ND & ND & ND & ND & ND & ND \\
\hline$\beta$-Hydroxyfentanyl & ND & ND & ND & $2.7 \pm 1.8$ & ND & ND & $1.1 \pm 0.2$ & $0.8 \pm 0.5$ \\
\hline Fentanyl & ND & ND & ND & ND & ND & ND & ND & $0.08 \pm 0.03$ \\
\hline Norfentanyl & ND & $2.0 \pm 0.4$ & $1.9 \pm 0.1$ & $1.8 \pm 0.2$ & ND & $0.5 \pm 0.1$ & $0.5 \pm 0.1$ & $0.5 \pm 0.03$ \\
\hline
\end{tabular}

a Pupae ( $\mathrm{n}=5)$ Collected from day 10 for each treatment group

b Pooled Pupa ( $\mathrm{n}=5)$ Pooled specimen of 4 pupa collected from day 10 for each treatment group

'T Treatments: Control $(0 \mu \mathrm{g} / \mathrm{kg}), \mathrm{Low}(6.0 \mu \mathrm{g} / \mathrm{kg})$, Medium $(23.3 \mu \mathrm{g} / \mathrm{kg})$, and High $(239 \mu \mathrm{g} / \mathrm{kg})$ quantitated from liver tissue collected at time of egg placemen

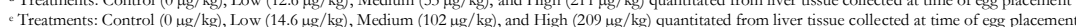




\subsubsection{Regression Plots}

To evaluate the correlation of the insect tissue concentration to liver concentration, simple linear regression plots were created to compare correlation. For best correlation, a minimum of three points are necessary to create a linear regression. Therefore, correlation plots were only created for the feeding larva extractions.

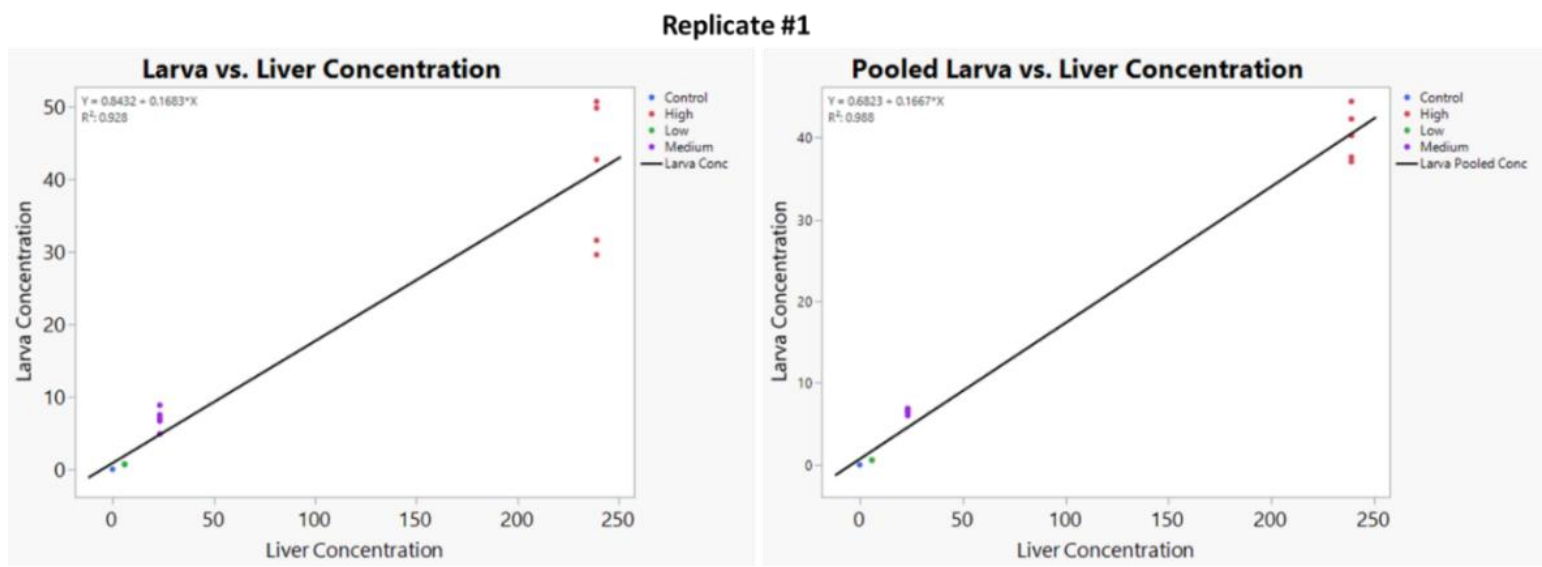

Figure 4.23: Regression plots for liver concentrations to larva concentrations for the single and pooled extractions collected on day 3 in replicate \#1.

The regression plots in figure 4.23 display the single and pooled larva concentrations to liver concentrations. The $R^{2}$ value for the single larva concentrations was 0.928 while the $R^{2}$ value for the pooled larva concentrations was higher at 0.988 . An $\mathrm{R}^{2}$ value of greater than 0.90 indicates a strong correlation, showing that the larva concentrations from replicate \#1 are strongly correlated to the liver tissue that the insects fed on.

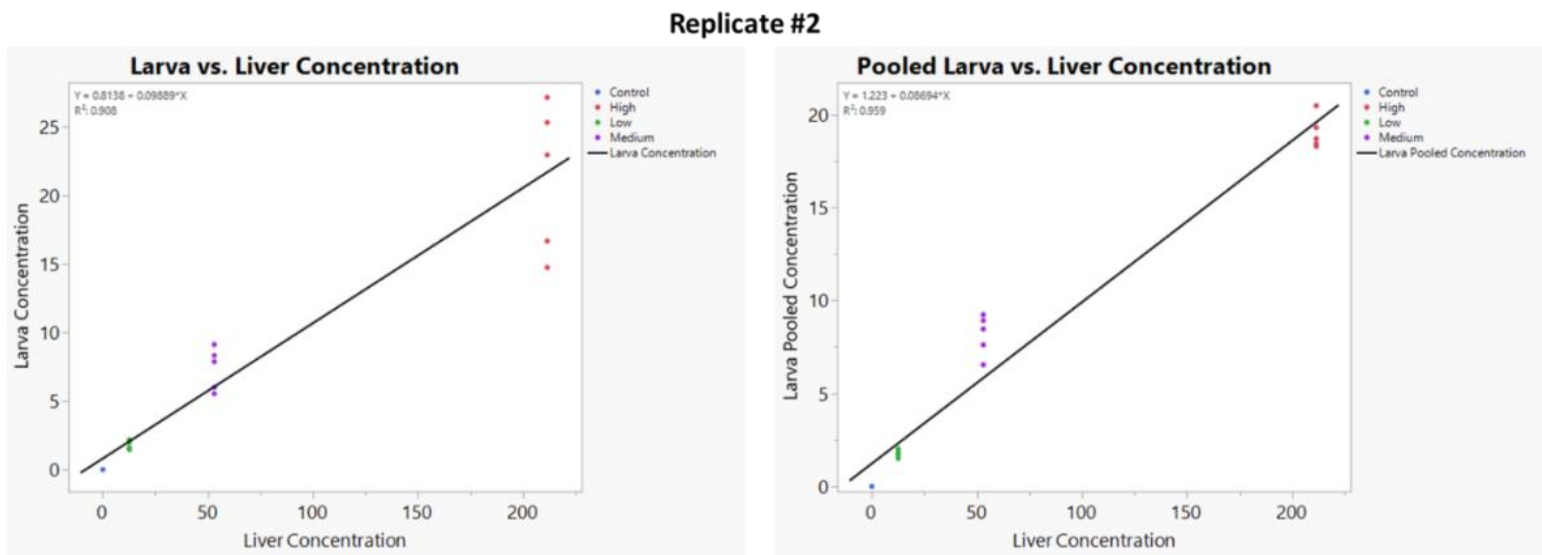

Figure 4.24: Regression plots for liver concentrations to larva concentrations for the single and pooled extractions collected on day 3 in replicate \#2. 
The regression plots in figure 4.24 display the single and pooled larva concentrations to liver concentrations. The $R^{2}$ value for the single larva concentrations was 0.908 while the $R^{2}$ value for the pooled larva concentrations was higher at 0.959 . With $\mathrm{R}^{2}$ values greater than 0.90 , the larva concentrations from replicate \#2 are strongly correlated to the liver tissue that the insects fed on.

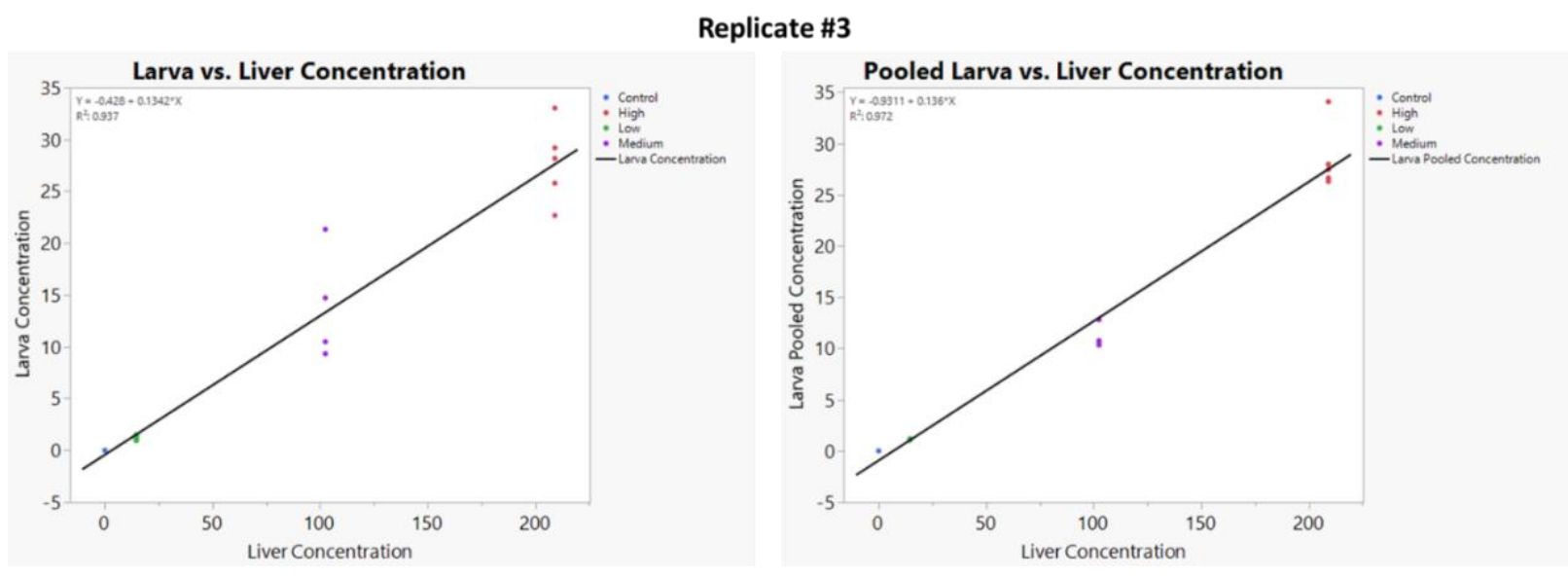

Figure 4.25: Regression plots for liver concentrations to larva concentrations for the single and pooled extractions collected on day 3 in replicate \#3.

The regression plots in figure 4.25 display the single and pooled larva concentrations to liver concentrations. The $R^{2}$ value for the single larva concentrations was 0.937 while the $R^{2}$ value for the pooled larva concentrations was higher at 0.972 . With $R^{2}$ values greater than 0.90 , the larva concentrations from replicate \#3 are strongly correlated to the liver tissue that the insects fed on. The larva collected from the authentic liver specimen were not included in the correlation plots for replicate \#3. Instead, this data was used to test the correlation of the larva concentration to liver concentrations for estimation of liver tissue concentration. The equation generated from the linear regression (Table 4.16) was used to estimate the liver concentration. 
Table 4.16: The linear regression equations for the regression plots with $\mathrm{R}^{2}$ values.

\begin{tabular}{ccc}
\hline Treatment & Equation & $\mathrm{R}^{2}$ \\
\hline Single Larva Replicate \#1 & $\mathrm{y}=0.1683 \mathrm{x}+0.8432$ & 0.928 \\
Pooled Larva Replicate \#1 & $\mathrm{y}=0.1667 \mathrm{x}+0.6823$ & 0.988 \\
Single Larva Replicate \#2 & $\mathrm{y}=0.0989 \mathrm{x}+0.8138$ & 0.908 \\
Pooled Larva Replicate \#2 & $\mathrm{y}=0.0869 \mathrm{x}+1.2233$ & 0.959 \\
Single Larva Replicate \#3 & $\mathrm{y}=0.1342 \mathrm{x}-0.428$ & 0.937 \\
Pooled Larva Replicate \#3 & $\mathrm{y}=0.136 \mathrm{x}-0.9299$ & 0.972 \\
Single Larva Total & $\mathrm{y}=0.1444 \mathrm{x}-0.0779$ & 0.890 \\
Pooled Larva Total & $\mathrm{y}=0.141 \mathrm{x}-0.1822$ & 0.930 \\
\hline
\end{tabular}

Using the equation generated from the linear regression of pooled larva concentration to liver concentration for replicate \#3 (Table 4.16), the quantitated larva concentration for the larva feeding on the authentic liver sample was used to estimate the liver concentration. Table 4.17 displays the results for that calculation. The average $(n=4)$ concentration of the authentic liver was quantitated at $111.4 \mu \mathrm{g} / \mathrm{kg}$. The single larva estimated the liver concentration ranging from 43.1 to $113.9 \mu \mathrm{g} / \mathrm{kg}$ with an average of $87.7 \mu \mathrm{g} / \mathrm{kg}$. The average bias $(\mathrm{n}=5)$ for the single larva estimates to the actual liver concentration was $21.7 \mu \mathrm{g} / \mathrm{kg}$. One larva was quantitated as a low outlier and if removed the average bias $(\mathrm{n}=4)$ becomes $11.8 \mu \mathrm{g} / \mathrm{kg}$. The pooled larva estimated the liver concentration ranging from 61.1 to $83.0 \mu \mathrm{g} / \mathrm{kg}$ with an average of $75.7 \mu \mathrm{g} / \mathrm{kg}$. The average bias $(n=5)$ for the single larva estimates to the actual liver concentration was $-32.1 \mu \mathrm{g} / \mathrm{kg}$. Both the single and the pooled liver concentrations using the linear regression underestimated the actual liver concentration. 
Table 4.17: Liver concentration estimation using larval concentration by linear regression.

\begin{tabular}{cccc}
\hline Sample & Larva Concentration & Estimated Liver Concentration & Bias \\
\hline Auth Single A & 14.5 & 113.3 & 1.7 \\
Auth Single B & 14.6 & 113.9 & 2.2 \\
Auth Single C & 10.3 & 82.3 & -26.1 \\
Auth Single D & 4.9 & 43.1 & -61.3 \\
Auth Single E & 10.5 & 83.7 & -24.9 \\
\hline Average & 10.9 & 87.3 & -21.7 \\
\hline Auth Pooled A & 7.4 & 61.1 & -45.1 \\
Auth Pooled B & 9.9 & 80.0 & -28.2 \\
Auth Pooled C & 10.3 & 82.4 & -26.0 \\
Auth Pooled D & 10.4 & 83.0 & -25.5 \\
Auth Pooled E & 8.9 & 72.0 & -35.3 \\
\hline Average & 9.4 & 75.7 & -32.1 \\
\hline
\end{tabular}

Total Replicates
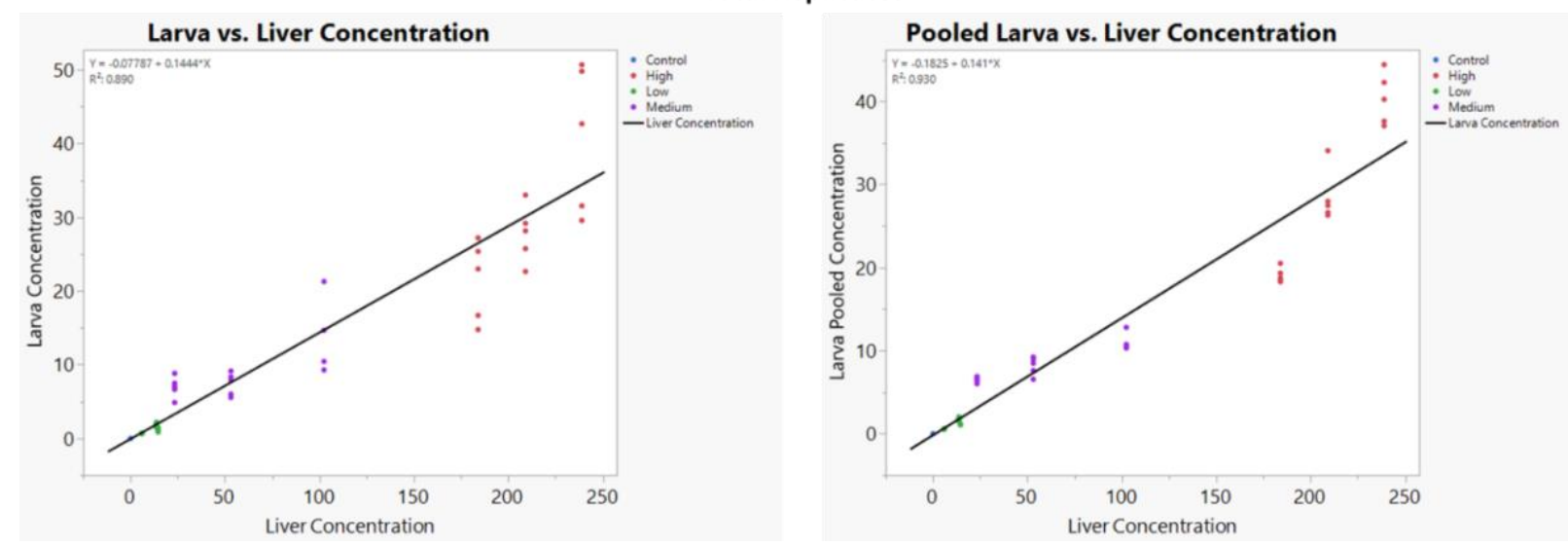

Figure 4.26: Correlation plots for liver concentrations to larva concentrations for the single and pooled extractions collected on day 3 for all three replicates. 
For an overall comparison, the entire set of replicate data of larva concentration to liver concentrations were plotted. The outcome is shown in figure 4.26 . The $R^{2}$ value for the single larva comparisons was the lowest of all the correlation plots at 0.890 and was the only one below 0.90 . The $\mathrm{R}^{2}$ value for the total pooled larva concentrations to liver concentrations was 0.930 . Both plots indicate a strong correlation for the larva concentration to the liver concentration of the treatment group. The $\mathrm{R}^{2}$ indicates that $93 \%$ of the variation in the larva fentanyl concentration can be explained by the liver fentanyl concentration.

\subsubsection{Discussion}

Fentanyl was quantitated in all larva from collection day three for all three treatments of low, medium, and high across the three replicates. These findings are similar to previous studies on opioids where drugs are found in higher concentration while the insects are still feeding $(45,54,103)$. By quantitating fentanyl in all three treatments, a correlation plot and simple linear regression was performed using the known concentrations of the fortified liver feeding substrate. Strong correlation of larva concentration to liver tissue concentration was observed for all replicates in the single and pooled larva specimens. The single larva to liver correlations observed an $\mathrm{R}^{2}$ value ranging from 0.908 to 0.937 and the pooled larva to liver correlations observed an $R^{2}$ value ranging from 0.959 to 0.988 . These strong correlation values are similar to those reported by El-Samad et al., who reported strong correlation values above 0.905 for tramadol dosed rabbit tissue fed on by L. sericata (45). To further investigate the utility of the correlation, the larva collected from the authentic treatment group was used to test the ability of the linear regression to estimate the liver fentanyl concentration using the larva fentanyl concentration. After estimation, the single larva fentanyl extractions had an average bias $(n=5)$ of -21.7 and the pooled specimens had an average bias $(n=5)$ of -32.1 . After dropping an outlier, the average bias $(n=4)$ for the single larva was -11.8 . It is not known what created the outlier, but this highlights why it is important to analyze multiple replicates for insect toxicology analysis or to sample a pooled specimen. The pooled 
insect specimens had less variation or standard deviations among the replicates and the means of the pooled specimens were not significantly different from the single samples for fentanyl. The fentanyl concentrations quantitated from the insects increased in concentration as the liver concentration increased producing a positive correlation however, there was little to no pattern for the metabolites detected. This is most likely due to the individual insect's ability to excrete the drug unmetabolized, metabolize the drug, or eliminate the metabolites from their bodies $(44,103)$.

The presented QuEChERS extraction and LC-MS/MS analysis was successful in quantifying fentanyl and metabolites from the insect tissue as well as the feeding media. Fentanyl and all three metabolites were detected in at least one treatment group of insects across the three replicates. With the analysis of the liver on the day of egg placement and collection of the insects not containing any of the three targeted metabolites of fentanyl, it can be derived that the metabolism of the fentanyl occurred in the insect and not in the feeding substrate. The extensive metabolism of fentanyl in humans has been well documented with most elimination driven by first pass hepatic metabolism (102). Primary metabolites generated by human metabolism of fentanyl are norfentanyl and despropionyl fentanyl (4-ANPP) with norfentanyl being the most abundant (102). Cytochrome P450 is highlighted as the primary actor in the first pass metabolism for fentanyl in human metabolic pathways (102).

Insects have an open circulatory system that means the blood of the insect is not contained by blood vessels but are contained in a cavity called a hemocoel and their blood is called hemolymph due to its mixing with other fluids (154). This allows for the potential broad recovery of drugs throughout the insect as the xenobiotics will not be enclosed in blood vessels (154). The excretory system of an insect consists of Malpighian tubules (MT) and the hindgut $(28,29)$. Each Malpighian tubule is a blind-ended tube comprised of a single-layer of squamous epithelial cells $(28,29)$. The MT are responsible for the transport of xenobiotics from within the insect's coelom to the hindgut for excretion (29). There is evidence of cytochrome P450 enzyme activity in the MT, resulting in metabolism of xenobiotics. The P450 
enzyme chemistry has been exploited to produce bioactivated insecticides where metabolism via the P450 enzyme creates a more toxic compound once metabolized in the insect $(28,29,154)$. This enzyme activity is potentially utilized when drugs are present in the insect as well, Maddrell and Gardiner reported Calliphora erythrocephala and Musca domestica when bathed in nicotine, the fluid secreted by the insects was a transformed compound other than nicotine (164). Thin layer chromatography (TLC) was used for the analysis and the transformed compound was not identified (164).

Specific drug metabolism mechanisms for blow fly species have not been thoroughly investigated and broad metabolic assumptions are difficult to ascertain. For Calliphora stygia, Parry et al. reported when maintained on meat containing morphine, the insects only contained morphine with no substantial metabolites detected (28). Excretion reported as clearance rate of morphine was reported indicating even without significant metabolism for the drug in the insect, elimination of the drug was still possible (28). In the pupal stage, morphine was reported as retained in the pupae when the Malpighian tubules are degraded and reformed as part of metamorphosis (28). Directly before and during metamorphosis, the morphine can be incorporated into the cuticle and excreted with the exuviae as the morphine in the empty puparium was found in a higher concentration compared to the adult fly (28). Bourel et al. found morphine excreted by epidermal cells by pore canals in the endocuticle and exocuticle (154). When the insect transitions from the third instar larval stage to the pupa stage, the cuticle is sclerotized, trapping the drugs in the puparium case in close proximity to the pore canals (154). This is evidence of deposition of drugs in the cuticle as a storage mechanism and could be evidence of a mechanism for how incorporation of drugs into the empty puparium occurs (154). Adult flies also excrete waste via meconium which is the first excrement of the adult once it emerges as an adult fly. This meconium is used to facilitate waste that accumulates during the pupa stage and if drugs are in this waste they can be excrete in this process that could explain a decrease of drugs detected in the adults compared to the empty puparia. 
The extraction of drugs from insects after they accumulated and incorporated drugs from tissues that they have fed on is not an undiscovered science $(39,50,111)$. Even though multiple classes of drugs have been investigated including opioids, very little if any information has been published on the extraction of fentanyl and metabolites from insects and the persistence of the drug through insect life stages $(39,54,144)$. Fentanyl is a highly effective $\mu$-opioid agonist with a potency of $50-100$ times that of morphine with considerable addictive potential but due to its potency is often detected in tissues at relatively low concentrations $(64,91,92)$. This is a challenge for toxicologists trying to use insects as a proxy to determine qualitative or quantitative drug presence in the tissue the insects feed on as insects often incorporate a smaller concentration of the drug compared to the feeding tissue $(39,45,50,103)$.

The QuEChERS extraction and LC-MS/MS analysis was able to extract and quantitate fentanyl and metabolites from pupa, feeding, and wandering larva. Extraction from insect tissue can be difficult due to their chitin exoskeleton, waxy cuticle, and fat bodies $(46,154,163)$. It is not uncommon for entomotoxicological studies to be unable to quantitate drugs or metabolites from low treatment concentrations or from some mid-life stages followed by detection of drugs in later life stages $(46,103$, 111). This study is not exempt from that either as the low treatment in the middle life stage insects produced no drug quantitation results. However, in every replicate for each life stage, fentanyl or metabolites were detected in either the single or pooled specimens highlighting the importance of using a selective and sensitive instrument to analysis of insects as a toxicological matrix.

\subsubsection{Conclusion}

Using a method validated following the ASB Standard 036 requirements, a QuEChERS extraction was used to quantitate fentanyl and metabolites from larval and pupal tissue. Fentanyl was detected in the larvae and pupae that were collected from the human liver tissue. A strong positive correlation for the liver to larvae concentrations was calculated and provides hope that larvae can be used in the future to, at minimum, determine the presence of fentanyl from feeding on human tissue and potentially 
estimate human tissue concentrations. A correlation from a specific tissue is not the same as a full human cadaver and metabolite and multidrug overdose deaths can be a complication for application to actual casework. Fentanyl and all three metabolites were detected in at least one treatment group in all three replicates. Metabolites of norfentanyl and $\beta$-hydroxyfentanyl were quantitated from the insect tissue when the presence of those metabolites were not detected in the liver at the time of egg placement nor at the time of collection. It can be derived that the most likely creation of these metabolites was metabolism of fentanyl by the insect to these compounds. Also, the metabolite 4-ANPP was quantitated from the feeding larva insects collected from the authentic liver specimen; however, this liver had a quantified 4-ANPP prior to egg placement and with no other insect containing a quantifiable concentration of 4-ANPP, no metabolism of fentanyl to 4-ANPP by the insects can be determined.

Overall, the QuEChERS extraction was successful at extracting fentanyl from larvae and pupae collected from feeding on human liver tissue that contained fentanyl. This provides a method for detecting and quantifying fentanyl and metabolites in an alternate matrix, liver to larvae concentrations, and shows the persistence of fentanyl and metabolites through the insect life cycle to pupation. 
Chapter 5: Evaluation of the Effects of Fentanyl on Blowfly Adults and Application of QuEChERS Extraction Method for the Quantitation of Fentanyl and Metabolites from Empty Puparium and Adult Flies (Lucilia sericata) 


\subsection{Chapter 5 Overview}

In this chapter, section 1 presents the validation of the extraction method for the analysis of fentanyl and metabolites from empty puparia and adult fly tissue for part of task 1.b. Also presented, is the preliminary study for the purpose of evaluating extraction methods for empty puparia with the target of choosing a method that efficiently recovers fentanyl and metabolites from the difficult matrix.

Section 2 of this chapter presents the morphometric comparisons for the adult flies collected in task 2.a. For all the insect stages, physical characteristics were recorded to evaluate the effects each treatment had on growth (task 2.b). To determine the effects of fentanyl on the growth and development of the blow flies, the mass, and percent stage of the insect were statistically compared using nested ANOVA (task 2.c). Tukey-HSD test will be applied to determine which treatments are significantly different within each replicate. These statistical tests will determine if the drug treatments have any significant effects on the insect's development.

Section 3 of this chapter presents the toxicological findings for the empty puparia and adult fly tissue for task 1.e. The presented modified QuEChERS extraction was used for extraction of the feeding media liver and the insect tissue. The survey of fentanyl and metabolites detected in the empty puparia and adult flies is presented in this section. For task 3.a, a correlation plot was created for a comparison of the fentanyl concentrations detected in the insect tissue to the liver fentanyl concentrations to establish any correlation of the concentrations.

\subsection{Section 1: Validation of QuEChERS Extraction for Empty Puparia and Adult Flies}

\subsubsection{Late Insect Stage Overview}

The previous chapter explored the utility of the larval and pupal stages in terms of toxicological matrices and this section will explore the later life stages of $L$. sericata of empty puparia and adult flies. Previous studies for the extraction of drugs and toxins from the later life stages of necrophagous flies include methamphetamine, codeine, methadone, amitriptyline, cadmium, and thallium $(44,46,103,163$, 
165). The value of insect specimens of puparia and empty puparia is rooted in the chitin exoskeleton that is resistant to environmental factors and allows for the evidence to remain at the scene for months and years after all other tissues used for toxicology analysis are no longer available $(44,163,166)$. When drugs or toxins are sequestered into the puparia cases that are left behind when an adult fly emerges, if a toxicologist can extract those deposited drugs, a snapshot of drugs present in the body while the insect was feeding after soft tissues and traditional biological fluids are not present $(44,163,165)$.

In this section, the validation of the extraction method for the analysis of fentanyl and metabolites empty puparia and adult fly tissue is presented. A preliminary study for the purpose of validating the extraction method with extraction from authentic insect samples and to evaluate three extraction techniques for the recovery of drug from authentic empty puparia specimens.

\subsubsection{Introduction}

After death, the natural decomposition process degrades or eliminates traditional toxicology biological matrices of blood, urine, and liver tissue increasing the opportunity for insects to be used as an alternative matrix $(39,46,50)$. The study or use of insects as an alternative toxicological matrix is generally accepted, however, the interpretation of the drugs detected is still up for debate $(39,44)$. With lack of sufficient information regarding metabolism, feeding behavior, and correlation of drugs found in the insect tissue to the human tissue, further research is necessary for conclusions on drugs detected in insect tissue in relation to human tissue concentrations $(44,111)$. When drugs are deposited into the chitin exoskeleton, this allows for the evidence to remain at the scene for months and years after all other tissues used for toxicology analysis are no longer available $(44,163,166)$. When an adult fly emerges, the puparia cases that are left behind can be extracted to provide a qualitative observation of drugs present in the body while the insect was feeding after soft tissues and traditional biological fluids are not present $(44,163,165)$. The larval and early stages of necrophagous flies are often investigated but the later life stages of pupa, empty puparia, and adult flies are underrepresented in the literature $(39,44)$. The empty puparia left behind when adult flies emerge are often low in mass and low in concentration compared to 
the tissue they fed on $(39,44)$. This requires sensitive and effective extraction techniques for the quantitation of fentanyl and metabolites from empty puparia.

To explore the utility of analysis of empty puparia, three extraction techniques were evaluated for the recovery of drug from authentic empty puparia specimens, ionization suppression/enhancement, recovery, and process efficiency were compared for the three techniques. The extraction that performed the best was selected for validation following the American Standards Board (ASB) 036 requirements (104).

\subsubsection{Methods}

\subsubsection{Adult colony}

Adult colonies of L. sericata were established in May 2018 from field collected specimens from Morgantown, West Virginia, USA. The collected insects were allowed to grow to adulthood and adults were identified as L. sericata by physical morphological characters (62). The colony was housed in BugDorm1 (MegaView Co., Ltd., Taiwan) screen cages $(30 \mathrm{~cm} \times 30 \mathrm{~cm} \times 30 \mathrm{~cm}$ ) stored in a Percival I36LLVLC8 incubator (Percival Scientific, Inc., Perry, lowa, USA) at $25^{\circ} \mathrm{C}\left( \pm 0.5^{\circ} \mathrm{C}\right)$ at $65 \%$ relative humidity ( $\pm 10 \%)$ with a 12:12 (L:D) photoperiod. Adults were given honey and water ad libitum and raw beef liver was provided for oviposition. New generations were separated from adults after oviposition to signal the beginning of the next generation and were moved to a new screen cage during emergence.

\subsubsection{Chemicals and materials}

Analytical grade hydrochloric acid $(\mathrm{HCl})$ was obtained from Fisher Scientific (Fair Lawn, NJ). Analytical grade sodium hydroxide $(\mathrm{NaOH})$ was purchased from Sigma-Aldrich (St. Louis, $\mathrm{MO})$. Optima ${ }^{\circ}$ LC-MS grade 1-chlorobutane was purchased from Fisher Scientific (Fair Lawn, NJ). Other chemicals, reagents and drugs used for this study are listed in the methods section 4.1.3. The LC-MS/MS instrument, mass spectrometry parameters (Table 4.1), and chromatographic separation (Figure 4.2) are listed in section 4.1 .3 as well. 
Blank human liver specimens were received from the West Virginia Human Gift Registry and were tested for the presence of fentanyl analogs before use. These liver specimens were used as the fortified liver specimens and were homogenized with a blender. All samples were stored at $-20^{\circ} \mathrm{C}$ prior to use for approximately 4 to 10 months. All specimens were de-identified from personal identifying information and this study was determined to be exempt from Institutional Review Board (IRB) review due to postmortem specimen use and lack of human subject involvement.

\subsubsection{Design for Authentic Insect Samples}

To evaluate the three extraction techniques, authentic empty puparia were created by placing 250 eggs on $75 \mathrm{~g}$ of fortified human liver tissue in two treatment groups at concentrations of $250 \mathrm{\mu g} / \mathrm{kg}$ and $0 \mu \mathrm{g} / \mathrm{kg}$ (control). After the insects completed their life cycle, the adult flies and empty puparia were collected for analysis. Each adult fly leaves behind a single empty puparium. From the control group, 110 empty puparia and adult flies were collected and from the $250 \mu \mathrm{g} / \mathrm{kg}$ treatment group 100 empty puparia and adult flies were collected. The purpose of these samples was to test the three extraction technique's ability to release fentanyl and metabolites from authentic empty puparia samples. The empty puparia were randomly pooled $(n=16)$ and allowed for each extraction technique to be run in duplicate.

To further test the chosen extraction protocol after validation, fentanyl was fortified into $10 \mathrm{~g}$ aliquots of human liver homogenate to evaluate four concentrations: control $(0 \mu \mathrm{g} / \mathrm{kg})$, low (50 $\mu \mathrm{g} / \mathrm{kg})$, medium $(250 \mu \mathrm{g} / \mathrm{kg})$, and high $(500 \mu \mathrm{g} / \mathrm{kg})$ concentrations in triplicate. To each aliquot of liver, approximately 35-50 eggs (by mass) were placed and allowed to feed undisturbed until time of collection. After the insects completed their life cycle (day 21), all empty puparia and adults from each replicate were collected, rinsed with deionized water followed by methanol, and dried before being stored at $-20^{\circ} \mathrm{C}$ with no liquid preservatives added $(7,40,46)$. No preservative was chosen due to the insect cuticle acting as a semipermeable membrane which can allow water or ethanol to diffuse into the body cavity and potential 
to leech out the target analytes into the preservative solution (149). The entire cohort for each replicate was pooled and extracted so no random selection was performed.

\subsubsection{Comparison of Extraction Techniques}

Three extraction techniques were tested for the extraction of fentanyl and metabolites from the empty puparia. The extraction that performed the best was selected for validation. To evaluate the extraction techniques, ionization suppression/enhancement, recovery, process efficiency, and the ability to extract from an authentic set of empty puparia collected from insects fed on liver tissue fortified with fentanyl were compared for the three techniques.

Due to small individual insect weights, the samples were pooled of 16 insects for triplicate analysis $(\mathrm{n}=3)$ with the results presented as $\mu \mathrm{g} / \mathrm{kg}$. Prior to the application of an extraction protocol, the pooled insects were pulverized using the mixer mill MM200 for a minimum of five minutes or until the insects were ground into a fine powder. This was critical to ensure the extraction protocols would be successful for extraction of fentanyl and metabolites. The micro pulverization method was similar to published studies for the extraction of drugs from hair $(123,127)$.

\subsection{Micropulverized QuEChERS Extraction}

The QuEChERS extraction slightly modified from the protocol in section 4.13 using a target sample aliquot of $0.2 \mathrm{~g}$ of empty puparia or adult flies as appropriate. Insect specimens were placed into a $1.5 \mathrm{~mL}$ centrifuge tube, followed by $400 \mu \mathrm{L}$ deionized water, $100 \mu \mathrm{L}$ internal standard mix (IS), and $700 \mu \mathrm{L}$ acetonitrile combined with three steel beads. The IS was comprised of three deuterated compounds: fentanyl- $D_{5}$, norfentanyl- $D_{5}$, and 4-ANPP- $D_{5}$ at a concentration of $1 \mathrm{ng} / \mathrm{mL}$. Afterwards, $0.2 \mathrm{~g}$ of QuEChERS original extraction salt was added to the tubes, vortexed, mixed on a mixer mill for 3 min, centrifuged for $5 \mathrm{~min}$, and the resulting supernatant was transferred to a QuEChERS dispersive-SPE tube. Tubes were vortexed then centrifuged for $5 \mathrm{~min}$ before the supernatant was transferred to a new $1.5 \mathrm{~mL}$ microcentrifuge tube for dry down under a nitrogen stream at $50{ }^{\circ} \mathrm{C}$ to dryness. Samples were reconstituted with $40 \mu \mathrm{L}$ methanol and transferred to vials for LC-MS/MS analysis. This extraction protocol 
was adapted and validated for larva and pupa tissue from work published by Cox et al. for the extraction of liver tissue (4).

\subsubsection{Acidic Digestion QuEChERS Extraction}

Samples of approximately $0.2 \mathrm{~g}$ (16 specimens) of pulverized empty puparia were placed into 1.5 $\mathrm{mL}$ centrifuge tubes with then $300 \mu \mathrm{L}$ of $1 \mathrm{M} \mathrm{HCl}$ and $100 \mu \mathrm{L}$ internal standard (IS). The tubes were vortexed and placed in a $80^{\circ} \mathrm{C}$ rotating heating block $(500 \mathrm{rpm})$ for 3 hours to incubate $(123,167)$. After incubation, the $\mathrm{pH}$ of the samples was alkalinized with $300 \mu \mathrm{L}$ of $1 \mathrm{M} \mathrm{NaOH}$. To each sample, $100 \mu \mathrm{L}$ internal standard mix (IS), $700 \mu \mathrm{L}$ acetonitrile, and three steel beads were added. Afterwards, $0.2 \mathrm{~g}$ of QuEChERS original extraction salt was added to the tubes, vortexed, mixed on a mixer mill for 3 min, centrifuged for 5 min, and the resulting supernatant was transferred to a QuEChERS dispersive-SPE tube. Tubes were vortexed then centrifuged for $5 \mathrm{~min}$ before the supernatant was transferred to a new $1.5 \mathrm{~mL}$ microcentrifuge tube for dry down under a nitrogen stream at $50{ }^{\circ} \mathrm{C}$ to dryness. Each sample was then reconstituted with $40 \mu \mathrm{L}$ of methanol and transferred to a LC-MS/MS vial for analysis.

\subsubsection{Basic Digestion and LLE}

Samples of approximately $0.2 \mathrm{~g}$ (16 specimens) of pulverized empty puparia were placed into 1.5 $\mathrm{mL}$ centrifuge tubes with then $200 \mu \mathrm{L}$ of $1 \mathrm{M} \mathrm{NaOH}$ and $100 \mu \mathrm{L}$ internal standard (IS). The tubes were vortexed and placed in a $80^{\circ} \mathrm{C}$ rotating heating block $(500 \mathrm{rpm})$ for 30 mins to incubate $(123,127)$. Then $200 \mu \mathrm{L}$ deionized water, $100 \mu \mathrm{L} \mathrm{IS}$, and $1 \mathrm{~mL}$ of 1-chlorobutane were added to the tubes. 1-chlorobutane was chosen as the organic solvent based on the successful extraction of methadone from empty puparia and fentanyl from hair in previous published $\operatorname{LLE}$ work $(44,123,127)$. The tubes were vortexed, rocked for 5 mins, and then centrifuged for 5 minutes. After centrifugation, the supernatant was transferred to a 1.5 $\mathrm{mL}$ microcentrifuge tube and the samples were evaporated under a nitrogen stream, at $50^{\circ} \mathrm{C}$, to dryness. The sample was reconstituted with $40 \mu \mathrm{L}$ of methanol and transferred to a LC-MS/MS vial for analysis. 


\subsubsection{Validation parameters}

The QuEChERS extraction and LC-MS/MS analysis was validated following the American Standards Board (ASB) 036 requirements (104). Evaluated parameters included calibration model, interference studies, ionization suppression/enhancement, dilution integrity, limits of quantitation, processed sample stability, bias, and precision.

\subsubsection{Calibration model}

Six different concentrations for five runs $(n=15)$ were analyzed for both empty puparia and adult fly tissue by linear regression lines generated using Agilent MassHunter software with a weight of (1/x). The linear range or analytical measurement range (AMR) is displayed in Table 5.3 for each analyte in both matrices. A calibration stock mix of fentanyl ( $2 \mathrm{ng} / \mu \mathrm{L})$, 4-ANPP $(2 \mathrm{ng} / \mu \mathrm{L})$, norfentanyl $(10 \mathrm{ng} / \mu \mathrm{L})$, and $\beta$ hydroxy fentanyl $(8 \mathrm{ng} / \mu \mathrm{L})$ was created for spiking tissues in the calibration curve and represents the highest calibrator. Using this solution, a serial dilution was performed to create stock solutions at concentrations of $0.002,0.004,0.008,0.04,0.2,1$, and $2 \mathrm{ng} / \mu \mathrm{L}(\mathrm{ppm})$ for fentanyl and 4-ANPP with concentrations of $0.01,0.02,0.04,0.2,1,5$, and $10 \mathrm{ng} / \mu \mathrm{L}(\mathrm{ppm})$ for norfentanyl and $0.006,0.012,0.032$, $0.16,0.8,4$, and $8 \mathrm{ng} / \mu \mathrm{L}(\mathrm{ppm})$ for $\beta$-hydroxy fentanyl. To each $0.2 \mathrm{~g}$ insect calibrator sample, $5 \mu \mathrm{L}$ of each stock solution was added to create the calibration curve. This created the analytical measurement range (AMR) of 0.05 to $50 \mu \mathrm{g} / \mathrm{kg}$ for fentanyl and 4-ANPP, 0.25 to $250 \mu \mathrm{g} / \mathrm{kg}$ for norfentanyl, and 0.2 to 200 $\mu \mathrm{g} / \mathrm{kg}$ for $\beta$-hydroxyfentanyl.

\subsubsection{Interference studies}

To evaluate interferences a high concentration (highest calibrator) of the target analytes was injected with no IS to show any interference of the drug standards to the IS. Conversely, an aliquot of IS with no target analytes was injected to observe any interference caused by the IS. Matrix interferences were evaluated by extracting blank matrix sources $(n=10)$ for both empty puparia and adult flies with no IS added. To evaluate potential interferences from commonly encountered compounds, a mix of common drugs encountered in toxicology ( $n=31)$ was injected (Table 4.2). 


\subsubsection{Ionization suppression/enhancement}

Ionization suppression or enhancement was evaluated at two concentrations, low and high, for ten replicates at both concentrations following the ASB Standard 036 requirements. To be deemed acceptable, the percent ionization suppression or enhancement shall not exceed $\pm 25 \%$ with a percent relative standard deviation (\%CV) not exceeding $20 \%$ (104). A post-extraction addition approach was utilized by comparing neat standards (Set 1 ) to blank matrix samples fortified with neat standard after extraction (Set 2). Set 1 consisted of neat standards prepared at low ( 2 times the LLOQ) and high (50\% of the highest calibrator) and were injected ten times to establish the mean peak area for each target analyte. Set 2 consisted of ten individual blank matrix sources (larva and pupa) in duplicate, for low ( $n=10)$ and high $(n=10)$ concentration, extracted and spiked with the low and high concentration, respectively, after extraction. The average area of each set $(\bar{X})$ was used to calculate the suppression or enhancement effects at each concentration for each analyte is as follows:

$$
\text { Ionization suppression or enhancement }(\%)=\left(\frac{\bar{X} \text { Area of set } 2}{\bar{X} \text { Area of set } 1}-1\right) * 100
$$

\subsubsection{Bias and Precision}

For bias and precision calculations, blank larval and pupal tissue samples were spiked and analyzed at low, medium, and high concentrations for fentanyl and metabolites in triplicate over five days $(n=15)$. Bias was calculated as a percent deviation of the extracted mean concentration from the theoretical concentration with an acceptable bias not exceeding $\pm 20 \%$. Precision was evaluated by percent standard deviation $(\% \mathrm{CV})$ of the mean at each concentration with the criteria of not exceeding $20 \%$.

\subsubsection{Limits}

For the evaluation of the limit of detection (LOD) and the lower limit of quantitation (LLOQ), blank matrix sources for larval and pupal tissue were spiked with concentrations at the lowest non-zero 
calibrator. Triplicates of each blank matrix sources were spiked at the lowest non-zero calibrator and analyzed over three days ( $n=9)$. The acceptable parameters were bias $( \pm 20 \%)$ and precision (\%CV $\leq 20 \%)$. For LOD, chromatographic acceptance was evaluated and deemed acceptable when signal to noise $(\mathrm{s} / \mathrm{n})$ ratios were above 3.3 and qualifier ratios did not exceed $\pm 20 \%$.

\subsection{Carryover and Dilution Integrity}

Blank matrix samples were injected directly after injections of samples three times greater than the highest calibrator in triplicate analysis to evaluate carryover. Carryover was not considered significant if present below $10 \%$ of the lowest calibrator (LLOQ). For dilution integrity, blank matrix samples from both sources were spiked at concentrations three times the highest calibrator in triplicate and extracted simultaneously with blank matrix only spiked with internal standard in triplicate. The resulting extract was diluted 1:5 (S:T) with the extracted blank matrix with internal standard $(5,106)$. The process was repeated over a period of five runs $(n=15)$ and the dilution integrity was deemed acceptable if bias did not exceed $\pm 20 \%$ and the precision (\%CV) did not exceed $20 \%$.

\subsection{Processed Sample Stability}

To determine the stability of processed samples for both larval and pupal tissue in the autosampler $\left(25^{\circ} \mathrm{C}\right)$, blank larva and pupa samples were spiked at low and high concentrations and extracted. Post extraction reconstituted samples, for low and high respectively, were pooled and aliquoted to LC-MS/MS vials. Triplicate analysis at time frames: $0,12,24,36,48,60$, and $72 \mathrm{hr}$ were performed for each concentration. Average peak area was calculated for each triplicate and compared to time zero. An acceptable bias threshold was set at $\pm 20 \%$.

\subsubsection{Recovery and Process Efficiency}

To calculate recovery of the extraction (RE) and process efficiency (PE) two formulas were utilized.

Variables in the formulas represent the mean peak areas for the neat solution (A), the mean peak areas for the standards spiked after extraction (B), and the mean peak areas for the standards spiked before extraction (C) (107). Recovery and process efficiency were at low and high concentrations for ten 
replicates $(n=10)$ for each matrix type. Ideally, a perfect recovery or process efficiency would be $100 \%$ and $\pm 20 \%$ of this value is desired while not exceeding a \%CV of $20 \%$. The ASB Standard 036 does not have a requirement for recovery or process efficiency and the $\pm 20 \%$ while not exceeding a $\% C V$ of $20 \%$ is a selfimposed guideline.

$$
\begin{aligned}
& R E(\%)=\frac{C}{B} * 100 \\
& P E(\%)=\frac{C}{A} * 100
\end{aligned}
$$

\subsubsection{Results}

\subsubsection{Comparison of Extraction Techniques}

Ionization suppression or enhancement, recovery, and process efficiency for the three extraction techniques were compared directly for the high concentration (Table 5.1). For fentanyl, norfentanyl, and 4-ANPP, the micropulverized QuEChERS extraction (MP) had an ionization suppression or enhancement of less than $\pm 3.5 \%$ while the acidic digestion QuEChERS extraction and the basic digestion LLE had percentages of less than $\pm 6.3 \%$ and $\pm 5.2 \%$, respectively. For $\beta$-hydroxyfentanyl, the acidic digestion produced a slightly better ionization suppression of $-15.0 \%$ compared to $-17.7 \%$ and $-16.0 \%$ for the micropulverized QuEChERS and basic digestion LLE, respectively. The recovery for the micropulverized QuEChERS extraction ranged from $95.3 \%$ to $97.9 \%$, the recovery for acidic digestion QuEChERS extraction ranged from $96.9 \%$ to $137.0 \%$, and the recovery for the basic digestion LLE ranged from $94.8 \%$ to $270.8 \%$. The overall process efficiency for the micropulverized QuEChERS extraction ranged from $78.4 \%$ to $100.9 \%$, the process efficiency for acidic digestion QuEChERS extraction ranged from $82.3 \%$ to $145.6 \%$, and the recovery for the basic digestion LLE ranged from $99.0 \%$ to $227.3 \%$. 
Table 5.1: Ionization suppression/enhancement, recovery, and process efficiency for the empty puparium tissue ( $n=10)$.

\begin{tabular}{|c|c|c|c|c|c|c|}
\hline \multirow{3}{*}{ Compound } & \multicolumn{2}{|c|}{ Ionization Suppression/Enhancement ${ }^{\mathrm{a}}$} & \multicolumn{2}{|c|}{ Recovery $^{\mathrm{a}}$} & \multicolumn{2}{|c|}{ Process Efficiency ${ }^{\mathrm{a}}$} \\
\hline & \multicolumn{2}{|c|}{ High } & \multicolumn{2}{|c|}{ High } & \multicolumn{2}{|c|}{ High } \\
\hline & Mean & $\mathrm{CV}$ & Mean & $\mathrm{CV}$ & Mean & $\mathrm{CV}$ \\
\hline \multicolumn{7}{|c|}{ Micropulverized QuEChERS } \\
\hline 4-ANPP & 3.5 & 8.7 & 97.5 & 9.2 & 100.9 & 8.7 \\
\hline$\beta$-Hydroxyfentanyl & -17.7 & 11.4 & 95.3 & 9.2 & 78.4 & 11.4 \\
\hline Fentanyl & 2.9 & 8.3 & 96.5 & 8.8 & 99.3 & 8.3 \\
\hline Norfentanyl & 3.2 & 8.2 & 97.9 & 8.3 & 100.9 & 8.2 \\
\hline \multicolumn{7}{|c|}{ Acidic Digestion QuEChERS } \\
\hline 4-ANPP & 6.3 & 7.4 & 137.0 & 7.0 & 145.6 & 7.4 \\
\hline$\beta$-Hydroxyfentanyl & -15.0 & 8.7 & 96.9 & 3.5 & 82.3 & 8.7 \\
\hline Fentanyl & 5.2 & 5.2 & 99.1 & 3.8 & 104.2 & 5.2 \\
\hline Norfentanyl & 4.9 & 5.6 & 100.3 & 4.3 & 105.3 & 5.6 \\
\hline \multicolumn{7}{|c|}{ Basic Digestion and LLE } \\
\hline 4-ANPP & 4.4 & 6.7 & 94.8 & 6.9 & 99.0 & 6.7 \\
\hline$\beta$-Hydroxyfentanyl & -16.0 & 64.4 & 270.8 & 64.1 & 227.3 & 64.4 \\
\hline Fentanyl & 4.8 & 5.5 & 95.3 & 5.7 & 99.8 & 5.5 \\
\hline Norfentanyl & 5.2 & 5.5 & 101.5 & 5.2 & 106.8 & 5.5 \\
\hline
\end{tabular}

In comparison of the three extraction techniques in the ability to extract fentanyl from authentic empty puparia specimens, the micropulverized QuEChERS extraction quantitated an average $(n=2)$ of 5.2 $\pm 0.9 \mu \mathrm{g} / \mathrm{kg}$, the acidic digestion QuEChERS produced an average of $4.9 \pm 1.2 \mu \mathrm{g} / \mathrm{kg}$, and the basic digestion LLE an average of $3.7 \pm 0.05 \mu \mathrm{g} / \mathrm{kg}$ (Table 5.2). Each of the three extraction techniques were effective at extracting the three metabolites with none of the techniques being efficient at extracting all three metabolites. The MP extraction was successful in extracting $\beta$-hydroxyfentanyl and norfentanyl with averages of $4.1 \pm 1.5 \mu \mathrm{g} / \mathrm{kg}$ and $2.2 \pm 0.3 \mu \mathrm{g} / \mathrm{kg}$, respectively. The acidic digestion QuEChERS was successful at extracting 4-ANPP with an average of $1.1 \pm 0.4 \mu \mathrm{g} / \mathrm{kg}$; however only detected $\beta$-hydroxyfentanyl in one of the replicates and did not detect norfentanyl. The basic digestion LLE technique detected norfentanyl at an average of $1.3 \pm 0.04 \mu \mathrm{g} / \mathrm{kg}$; however, did not detect 4-ANPP or $\beta$-hydroxyfentanyl. For the L5 quality 
control, the MP technique performed the best with the bias ranging from $-7.6 \%$ to $-13.6 \%$ while the acidic digestion technique bias ranged from $-3.7 \%$ to $-21.0 \%$ and the basic digestion LLE bias ranged from $-3.5 \%$ to $-22.4 \%$.

Table 5.2: Comparison for the three extraction techniques micropulverized QuEChERS extraction (MP), acidic digestion QuEChERS (acidic), and basic digestion LLE for the ability to extract fentanyl and metabolites from pooled ( $n=16)$ authentic empty puparia specimens.

\begin{tabular}{|c|c|c|c|c|c|c|}
\hline Compound & & Fentanyl & & \multicolumn{3}{|c|}{$\beta$-Hydroxyfentanyl } \\
\hline Extraction Technique & MP & Acidic & Basic & MP & Acidic & Basic \\
\hline Replicate A & 4.5 & 5.8 & 3.7 & 3.0 & ND & ND \\
\hline Replicate B & 5.8 & 4.1 & 3.8 & 5.2 & 3.8 & ND \\
\hline Average & $5.2 \pm 0.9$ & $4.9 \pm 1.2$ & $3.7 \pm 0.05$ & $4.1 \pm 1.5$ & - & - \\
\hline L5 QC & 4.6 & 4.8 & 4.3 & 18.5 & 15.8 & 15.5 \\
\hline Bias & -7.8 & -3.7 & -14.1 & -7.6 & -21.0 & -22.4 \\
\hline Control & ND & ND & ND & ND & ND & ND \\
\hline Compound & \multicolumn{3}{|c|}{ 4-ANPP } & \multicolumn{3}{|c|}{ Norfentanyl } \\
\hline Extraction Technique & MP & Acidic & Basic & MP & Acidic & Basic \\
\hline Replicate A & ND & 1.3 & ND & 2.0 & ND & 1.3 \\
\hline Replicate B & ND & 0.8 & ND & 2.4 & ND & 1.3 \\
\hline Average & - & $1.1 \pm 0.4$ & - & $2.2 \pm 0.3$ & - & $1.3 \pm 0.04$ \\
\hline L5 QC & 4.5 & 4.3 & 4.8 & 21.6 & 21.7 & 21.0 \\
\hline Bias & -9.5 & -15.0 & -3.5 & -13.6 & -13.2 & -16.0 \\
\hline Control & ND & ND & ND & ND & ND & ND \\
\hline
\end{tabular}

\subsubsection{Validation}

The linear range or analytical measurement range (AMR) is displayed in Table 5.3 for each analyte in both matrices. As part of the evaluation of the calibration model, the $R^{2}$ values for the calibration curves exceeded 0.99 and the residuals for each set of five replicates were plotted with no discernable pattern being identified indicating a random dispersion. This allows for linear analysis of the analytes presented using a weight of $(1 / x)$. 
Table 5.3: LOD, LLOQ, and AMR for empty puparia and adult fly extractions.

\begin{tabular}{|c|c|c|c|c|}
\hline \multicolumn{5}{|c|}{ Empty Puparia } \\
\hline \multirow{2}{*}{ Compound } & \multirow{2}{*}{$\begin{array}{l}\text { LOD/LLOQ } \\
(\mu \mathrm{g} / \mathrm{kg})\end{array}$} & \multicolumn{2}{|c|}{ LLOQ $(n=9)$} & \multirow{2}{*}{$\begin{array}{c}\text { AMR } \\
(\mu \mathrm{g} / \mathrm{kg})\end{array}$} \\
\hline & & Bias & $\% \mathrm{CV}$ & \\
\hline 4-ANPP & 0.05 & 3.1 & 12.7 & $0.05-50$ \\
\hline$\beta$-Hydroxyfentanyl & 0.2 & -2.8 & 10.3 & $0.2-200$ \\
\hline Fentanyl & 0.05 & 13.0 & 4.7 & $0.05-50$ \\
\hline Norfentanyl & 0.25 & 19.8 & 3.4 & $0.25-250$ \\
\hline \multicolumn{5}{|c|}{ Adult } \\
\hline \multirow{2}{*}{ Compound } & \multirow{2}{*}{$\begin{array}{l}\text { LOD/LLOQ } \\
\quad(\mu \mathrm{g} / \mathrm{kg})\end{array}$} & \multicolumn{2}{|c|}{ LLOQ (n=9) } & AMR \\
\hline & & Bias & $\% \mathrm{CV}$ & $(\mu \mathrm{g} / \mathrm{kg})$ \\
\hline 4-ANPP & 0.05 & 10.4 & 11.7 & $0.05-50$ \\
\hline$\beta$-Hydroxyfentanyl & 0.2 & -1.9 & 14.2 & $0.2-200$ \\
\hline Fentanyl & 0.05 & 12.5 & 7.9 & $0.05-50$ \\
\hline Norfentanyl & 0.25 & 19.4 & 2.4 & $0.25-250$ \\
\hline
\end{tabular}

Blank matrix samples from 10 individual insect sources for both larval and pupal tissue were extracted without addition of standards or internal standards to determine any matrix interferences. Chromatographic analysis of these injections was performed using Agilent MassHunter Qualitative Analysis software version B.08.00 and no interference peaks from the blank matrices were observed.

No interfering peaks were observed from the high standards to the internal standards or from the internal standards to the target analytes. A neat mix of 31 commonly encountered analytes, encompassing other opioids, stimulants, depressants, synthetic cannabinoids, synthetic cathinones, and dietary supplements, was injected and no interferences were observed.

\subsubsection{Ionization suppression/enhancement}

lonization suppression or enhancement was evaluated at low and high concentrations $(n=10)$ for both empty puparia and adult fly tissue following the ASB Standard 036 requirements (168). The percent ionization suppression or enhancement and \%CV for all four target analytes is presented in Table 5.4. The 
four target analytes were within the criteria for acceptance at both high and low concentrations for both insect tissue types. For the empty puparia tissue, the percent ionization suppression or enhancement for the four analytes ranged from $-9.1 \%$ to $5.6 \%$ for the low and ranged from $-17.7 \%$ to $3.5 \%$ for the high while not exceeding a \%CV of $11.4 \%$. For the adult fly tissue, the percent ionization suppression or enhancement ranged from $4.5 \%$ to $9.2 \%$ at the low concentration and ranged from $3.6 \%$ to $11.8 \%$ for the high while not exceeding a \%CV of $10.9 \%$.

Table 5. 4: Ionization suppression/enhancement, recovery, and process efficiency for the adults and empty puparium tissue $(n=10)$.

\begin{tabular}{|c|c|c|c|c|c|c|c|c|c|c|c|c|}
\hline \multirow{3}{*}{ Compound } & \multicolumn{4}{|c|}{$\begin{array}{c}\text { Ionization } \\
\text { Suppression/Enhancement }^{\mathrm{a}} \\
\end{array}$} & \multicolumn{4}{|c|}{ Recovery $^{\mathrm{a}}$} & \multicolumn{4}{|c|}{ Process Efficiency ${ }^{a}$} \\
\hline & \multicolumn{2}{|c|}{ Low } & \multicolumn{2}{|c|}{ High } & \multicolumn{2}{|c|}{ Low } & \multicolumn{2}{|c|}{ High } & \multicolumn{2}{|c|}{ Low } & \multicolumn{2}{|c|}{ High } \\
\hline & Mean & $\mathrm{CV}$ & Mean & $\mathrm{CV}$ & Mean & $\mathrm{CV}$ & Mean & $\mathrm{CV}$ & Mean & $\mathrm{CV}$ & Mean & $\mathrm{CV}$ \\
\hline \multicolumn{13}{|c|}{ Adults } \\
\hline 4-ANPP & 8.9 & 10.6 & 9.2 & 9.9 & 90.9 & 9.6 & 91.7 & 6.2 & 99.1 & 12.0 & 100.1 & 10.6 \\
\hline $\begin{array}{c}\beta- \\
\text { Hydroxyfentanyl }\end{array}$ & 4.5 & 6.6 & 3.6 & 8.9 & 93.9 & 10.6 & 96.5 & 5.5 & 98.1 & 10.0 & 100.0 & 9.7 \\
\hline Fentanyl & 6.1 & 9.9 & 11.8 & 9.8 & 95.3 & 8.2 & 91.7 & 5.6 & 101.1 & 10.1 & 102.5 & 10.3 \\
\hline Norfentanyl & 9.2 & 10.9 & 8.0 & 9.8 & 95.1 & 9.9 & 91.9 & 5.5 & 103.9 & 12.2 & 99.3 & 10.7 \\
\hline \multicolumn{13}{|c|}{ Empty Puparium } \\
\hline 4-ANPP & -9.1 & 11.4 & 3.5 & 8.7 & 104.1 & 8.9 & 97.5 & 9.2 & 94.5 & 10.0 & 100.9 & 8.7 \\
\hline $\begin{array}{c}\beta- \\
\text { Hydroxyfentanyl }\end{array}$ & -10.0 & 11.3 & -17.7 & 11.4 & 107.6 & 9.0 & 95.3 & 9.2 & 96.8 & 9.7 & 78.4 & 11.4 \\
\hline Fentanyl & -10.4 & 9.3 & 2.9 & 8.3 & 109.0 & 8.5 & 96.5 & 8.8 & 97.6 & 7.2 & 99.3 & 8.3 \\
\hline Norfentanyl & 5.6 & 10.2 & 3.2 & 8.2 & 98.1 & 12.0 & 97.9 & 8.3 & 103.6 & 10.9 & 100.9 & 8.2 \\
\hline
\end{tabular}

\subsubsection{Bias and precision}

The bias for all four analytes in both insect matrices were within $\pm 20 \%$ and the $\% \mathrm{CV}$ did not exceed 20\%, meeting the ASB Standard 036 requirements (Table 5.5). For the empty puparia tissue, the bias for the low ranged from $0.6 \%$ to $5.4 \%$, the medium ranged from $-9.7 \%$ to $-1.5 \%$, and the high ranged from $7.1 \%$ to $-1.5 \%$. For the adult fly tissue, the bias for the low ranged from $0.6 \%$ to $4.3 \%$, for the medium ranged from $-11.5 \%$ to $-5.0 \%$, and for the high ranged from $-7.3 \%$ to $-5.1 \%$. Of the four analytes and two 
matrices, only norfentanyl and 4-ANPP experienced a bias greater than $\pm 10 \%$ at $-10.1 \%$ bias for the medium norfentanyl in the adult fly matrix and $-11.5 \%$ bias for the medium 4-ANPP in the adult fly matrix. These elevated percent bias values are still well within the criteria for acceptance.

Table 5.5: The bias and precision validation data for adults and empty puparium extractions $(n=15)$.

\begin{tabular}{|c|c|c|c|c|c|c|c|c|c|}
\hline \multicolumn{10}{|c|}{ Adults } \\
\hline \multirow{2}{*}{ Compound } & \multicolumn{3}{|c|}{ Bias (\%) } & \multicolumn{3}{|c|}{ Between Run (\%CV) } & \multicolumn{3}{|c|}{ Within Run (\%CV) } \\
\hline & LOW & MED & HIGH & LOW & MED & HIGH & LOW & MED & $\mathrm{HIGH}$ \\
\hline 4-ANPPa & 0.6 & -11.5 & -6.3 & 9.1 & 6.1 & 3.6 & 4.6 & 3.7 & 4.0 \\
\hline$\beta$-Hydroxyfentanyl ${ }^{\mathrm{b}}$ & 2.4 & -5.0 & -7.1 & 11.9 & 7.4 & 3.9 & 6.3 & 3.7 & 3.4 \\
\hline Fentanyl $^{\mathrm{a}}$ & 1.8 & -8.7 & -7.3 & 6.8 & 6.2 & 3.4 & 5.9 & 4.0 & 3.7 \\
\hline Norfentanyl $^{\mathrm{c}}$ & 4.3 & -10.1 & -5.1 & 4.9 & 4.5 & 4.3 & 2.8 & 3.1 & 4.7 \\
\hline \multicolumn{10}{|c|}{ Empty Puparium } \\
\hline \multirow{2}{*}{ Compound } & \multicolumn{3}{|c|}{$\operatorname{Bias}(\%)$} & \multicolumn{3}{|c|}{ Between Run (\%CV) } & \multicolumn{3}{|c|}{ Within Run (\%CV) } \\
\hline & LOW & MED & HIGH & LOW & MED & HIGH & LOW & MED & HIGH \\
\hline 4-ANPPa & 0.7 & -5.6 & -6.1 & 5.6 & 5.8 & 3.2 & 5.2 & 4.8 & 3.2 \\
\hline$\beta$-Hydroxyfentanyl ${ }^{\mathrm{b}}$ & 0.6 & -1.5 & -6.3 & 7.0 & 4.1 & 3.4 & 6.3 & 4.4 & 3.1 \\
\hline Fentanyl $^{\mathrm{a}}$ & 5.4 & -5.3 & -7.1 & 5.8 & 4.7 & 3.3 & 5.3 & 4.9 & 3.3 \\
\hline Norfentanyl ${ }^{\mathrm{c}}$ & 6.0 & -9.7 & -1.8 & 9.3 & 6.5 & 5.0 & 6.2 & 5.2 & 3.2 \\
\hline
\end{tabular}

${ }^{\mathrm{a}}$ Low concentration at $0.2 \mu \mathrm{g} / \mathrm{kg}$, medium concentration at $5 \mu \mathrm{g} / \mathrm{kg}$, and high concentration at $40 \mu \mathrm{g} / \mathrm{kg}$

${ }^{\mathrm{b}}$ Low concentration at $0.8 \mu \mathrm{g} / \mathrm{kg}$, medium concentration at $20 \mu \mathrm{g} / \mathrm{kg}$, and high concentration at $160 \mu \mathrm{g} / \mathrm{kg}$

c Low concentration at $1.0 \mu \mathrm{g} / \mathrm{kg}$, medium concentration at $25 \mu \mathrm{g} / \mathrm{kg}$, and high concentration at $200 \mu \mathrm{g} / \mathrm{kg}$

\subsubsection{Limits}

Bias and precision for the LLOQ (Table 5.3) were considered acceptable within $\pm 20 \%$ for bias and the \%CV not exceeding $20 \%$ (104). The bias for the LLOQ for the empty puparia tissue ranged from $-2.8 \%$ to $19.8 \%$ and for the adult fly tissue ranged from $-1.9 \%$ to $19.4 \%$. The LOD was evaluated as the lowest calibrator which is also the LLOQ and the requirements for the LOD and LLOQ by the ASB Standard 036 were met for both matrices (104).

\subsubsection{Carryover and dilution integrity}

To determine any carryover, extracted blank matrix samples were injected directly after injections of samples with concentrations three times greater than the highest calibrator in triplicate analysis. This 
was performed for both the empty puparia and adult fly matrices. No significant carryover of the target analytes was detected in the blank matrix injections for either matrix.

For the dilution integrity evaluation, bias and precision was calculated for each analyte and is shown in Table 5.6. For the four target analytes in the empty puparia tissue the dilution integrity did not exceed $\pm 8.4 \%$ for bias and did not exceed $10.5 \%$ for $\% C V$. For the adult fly tissue, the dilution integrity did not exceed $\pm 4.4 \%$ for bias and did not exceed $17.2 \%$ for $\% C V$. For the empty puparia extractions, the bias of the dilution integrity ranged from $-8.4 \%$ to $-4.3 \%$ indicating a tendency for the dilution to have a lower than anticipated value. In contrast, the dilution integrity bias for the adult fly extracts ranged from $-0.7 \%$ to $4.4 \%$ indicating a slightly inflated value than expected. This could be attributed to matrix effect at the high concentration for the adult fly extraction as they experience a slight high-end ionization enhancement.

Table 5.6: Dilution integrity for the adult and empty puparium extractions at a 1:5 dilution.

\begin{tabular}{cccc}
\hline & Adult & & \\
\hline Compound & Target Concentration $(\mu \mathrm{g} / \mathrm{kg})$ & Dilution Integrity $(\%$ Bias $)$ & $\% \mathrm{CV}$ \\
\hline 4-ANPP & 150 & 4.4 & 13.3 \\
$\beta$-Hydroxyfentanyl & 600 & 1.8 & 17.2 \\
Fentanyl & 150 & -0.7 & 14.0 \\
Norfentanyl & 750 & 3.0 & 14.7 \\
\hline Compound & Empty Puparium & & $\%$ CV \\
\hline 4-ANPP & Target Concentration $(\mu \mathrm{g} / \mathrm{kg})$ & Dilution Integrity $(\%$ Bias $)$ & 10.5 \\
$\beta$-Hydroxyfentanyl & 150 & -4.9 & -7.4 \\
Fentanyl & 600 & -8.4 & 9.2 \\
Norfentanyl & 150 & -4.3 & 9.9 \\
\hline
\end{tabular}

\subsubsection{Processed sample stability}

Processed sample stability in the autosampler was evaluated for both insect matrices in triplicate at low and high concentrations over time intervals up to 72 hours (Table 5.7). For the empty puparia extractions, all four analytes were stable up to $72 \mathrm{~h}$ at the high concentrations but the $\beta$-hydroxyfentanyl stability exceeded $\pm 20 \%$ with $38.4 \%$ at the $72 \mathrm{~h}$ for the low concentration stability. It was determined that 
stability was maintained up to $60 \mathrm{~h}$ for that analyte for the low concentration. All the analytes at the low concentration were increasing and approaching the limit at $72 \mathrm{~h}$ for the empty puparia. For the adult fly extractions, all the analytes were stable up to $72 \mathrm{~h}$ for the high concentrations. For the low concentrations of the adult fly stability extractions, norfentanyl reached instability at $48 \mathrm{~h}$ and continued to exceed $20 \%$ in the next two time segments. Norfentanyl in the adult fly extractions was deemed stable only up to 36 $\mathrm{h}$ in the low concentrations.

Table 5. 7: Stability at a low and high concentration for the adult and empty puparium extractions for processed samples stored in the autosampler $\left(25^{\circ} \mathrm{C}\right)$.

\begin{tabular}{|c|c|c|c|c|c|c|c|c|c|c|c|c|}
\hline \multicolumn{13}{|c|}{ Adults } \\
\hline \multirow{2}{*}{ Compound } & \multicolumn{6}{|c|}{ Low $\left(\% \Delta^{\mathrm{d}}\right)$} & \multicolumn{6}{|c|}{$\operatorname{High}\left(\% \Delta^{\mathrm{d}}\right)$} \\
\hline & $12 \mathrm{~h}$ & $24 \mathrm{~h}$ & $36 \mathrm{~h}$ & $48 \mathrm{~h}$ & $60 \mathrm{~h}$ & $72 \mathrm{~h}$ & $12 \mathrm{~h}$ & $24 \mathrm{~h}$ & $36 \mathrm{~h}$ & $48 \mathrm{~h}$ & $60 \mathrm{~h}$ & $72 \mathrm{~h}$ \\
\hline 4-ANPPa & 0.3 & -3.7 & -0.7 & -2.0 & -2.7 & -2.6 & 0.4 & -1.7 & -0.4 & -1.7 & -3.5 & -4.7 \\
\hline$\beta$-Hydroxyfentanyl ${ }^{\mathrm{b}}$ & -3.8 & -4.5 & 4.7 & -2.4 & -1.6 & -1.1 & -1.6 & -1.9 & 0.0 & -1.4 & -1.6 & -1.6 \\
\hline Fentanyl $^{\mathrm{a}}$ & -3.9 & -1.6 & -2.6 & -1.6 & -3.7 & -2.3 & -0.7 & -1.8 & -0.8 & -2.6 & -3.4 & -4.4 \\
\hline Norfentanyl $^{\mathrm{c}}$ & 6.7 & 9.3 & 16.5 & 20.7 & 24.4 & 24.8 & -0.7 & -2.1 & -3.1 & -3.3 & -5.5 & -6.3 \\
\hline \multicolumn{13}{|c|}{ Empty Puparium } \\
\hline \multirow[t]{2}{*}{ Compound } & \multicolumn{6}{|c|}{ Low $\left(\% \Delta^{\mathrm{d}}\right)$} & \multicolumn{6}{|c|}{$\operatorname{High}\left(\% \Delta^{\mathrm{d}}\right)$} \\
\hline & $12 \mathrm{~h}$ & $24 \mathrm{~h}$ & $36 \mathrm{~h}$ & $48 \mathrm{~h}$ & $60 \mathrm{~h}$ & $72 \mathrm{~h}$ & $12 \mathrm{~h}$ & $24 \mathrm{~h}$ & $36 \mathrm{~h}$ & $48 \mathrm{~h}$ & $60 \mathrm{~h}$ & $72 \mathrm{~h}$ \\
\hline 4-ANPP & $\begin{array}{ll}-1.4 \\
\end{array}$ & -1.0 & -1.5 & -1.5 & -5.3 & -12.9 & -0.8 & -2.9 & -0.8 & -2.2 & -3.9 & -4.9 \\
\hline$\beta$-Hydroxyfentanyl ${ }^{\mathrm{b}}$ & -3.3 & -2.7 & 0.7 & 0.7 & 2.2 & 38.4 & -0.3 & -0.3 & 0.0 & 0.1 & 0.6 & -0.7 \\
\hline Fentanyl $^{\mathrm{a}}$ & -2.0 & -4.2 & -2.9 & -2.9 & -5.1 & 18.1 & -1.0 & -1.3 & -1.3 & -3.0 & -3.0 & -4.8 \\
\hline Norfentanyl $^{\mathrm{c}}$ & 5.3 & 9.7 & 12.7 & 12.7 & 12.3 & -15.6 & -1.6 & -1.9 & -3.2 & -2.9 & -4.7 & -6.6 \\
\hline $\begin{array}{l}\text { a Low concentration at } \\
\text { b Low concentration at } \\
\text { c Low concentration at }\end{array}$ & $\begin{array}{l}\mathrm{ig} / \mathrm{kq} \\
\mathrm{ug} / \mathrm{kq} \\
/ \mathrm{kg}\end{array}$ & $\begin{array}{l}\text { nd hig } \\
\text { nd his } \\
\text { high }\end{array}$ & concer & ation & $\begin{array}{l}25 \mu \mathrm{g} / \mathrm{l} \\
100 \mu \mathrm{g} \\
5 \mu \mathrm{g} / \mathrm{k}\end{array}$ & & & & & & & \\
\hline
\end{tabular}

\subsubsection{Recovery and process efficiency}

Recovery and process efficiency was evaluated simultaneously with ionization suppression or enhancement and the results are displayed in Table 5.4. The recoveries and process efficiencies for both matrices at the low and high concentrations were deemed acceptable within $\pm 20 \%$ of the target recoveries 
(100\%) and the $\% \mathrm{CV}$ not exceeding $20 \%$. Overall, the empty puparia extractions recovered more efficiently compared to the adult fly extractions at both concentrations. Conversely, the adult extractions obtain higher process efficiencies compared to the empty puparia extractions at both concentrations. The precision for the recovery and process efficiency did not exceed $12.0 \%$ with the majority being below $11 \%$.

\subsubsection{Entomotoxicology Results}

After fortifying liver tissue with varying fentanyl concentrations $(0,50,250$, and $500 \mathrm{ug} / \mathrm{kg})$ in triplicate analysis, the insects were allowed to finish their life cycle and the end of end stages of empty puparia and adult flies were collected. The empty puparia and adult flies collected from each collection day were treated as individual cohorts and all the insects were pooled for QuEChERS extraction of fentanyl and metabolites (Table 5.8). The average number of insects in a collected cohort was 26 insects. Each adult when emerging leaves behind an empty puparium, therefore each insect provides an adult fly and an empty puparium specimen. From the empty puparia specimens collected, fentanyl and norfentanyl was detected in all the treatments except for the control liver tissue spike. The average fentanyl concentrations $(n=3)$ for the pooled cohort of empty puparia collected were $0.1 \pm 0.1 \mu \mathrm{g} / \mathrm{kg}$ in the low treatment, $0.5 \pm 0.2 \mu \mathrm{g} / \mathrm{kg}$ in the medium treatment, and $1.1 \pm 0.4 \mu \mathrm{g} / \mathrm{kg}$ in the high treatment. The average norfentanyl concentrations $(n=3)$ for the pooled cohort of empty puparia collected were $0.4 \pm 0.1$ $\mu \mathrm{g} / \mathrm{kg}$ in the low treatment, $0.5 \pm 0.1 \mu \mathrm{g} / \mathrm{kg}$ in the medium treatment, and $0.9 \pm 0.2 \mu \mathrm{g} / \mathrm{kg}$ in the high treatment. From the adult fly specimens collected, fentanyl was detected in all the treatments except for the control liver tissue spike. The average fentanyl concentrations $(n=3)$ for the pooled cohort of adult flies collected were $0.1 \pm 0.04 \mu \mathrm{g} / \mathrm{kg}$ in the low treatment, $3.0 \pm 3.6 \mu \mathrm{g} / \mathrm{kg}$ in the medium treatment, and $1.8 \pm 1.6 \mu \mathrm{g} / \mathrm{kg}$ in the high treatment. From the adult flies collected, norfentanyl was only detected in the medium and high treatment groups. Average fentanyl concentrations $(n=3)$ for the adult flies collected from the medium treatment were $0.6 \pm 0.2 \mathrm{ug} / \mathrm{kg}$ and from the high treatment group $0.5 \pm 0.2 \mu \mathrm{g} / \mathrm{kg}$. 
Table 5.8: Concentrations of fentanyl and norfentanyl detected in the empty puparia and adult flies.

\begin{tabular}{cccccc}
\hline & \multicolumn{2}{c}{ Empty Puparia } & & \multicolumn{2}{c}{ Adult Flies } \\
\cline { 2 - 3 } \cline { 5 - 6 } Compound & Fentanyl & Norfentanyl & & Fentanyl & Norfentanyl \\
\hline Control & ND & ND & & ND & ND \\
Low & $0.1 \pm 0.09$ & $0.4 \pm 0.1$ & & $0.1 \pm 0.04$ & ND \\
Medium & $0.5 \pm 0.2$ & $0.5 \pm 0.1$ & & $3.0 \pm 3.6$ & $0.6 \pm 0.2$ \\
High & $1.1 \pm 0.4$ & $0.9 \pm 0.2$ & & $1.8 \pm 1.6$ & $0.5 \pm 0.2$ \\
\hline $\mathrm{n}=3$ & & & &
\end{tabular}
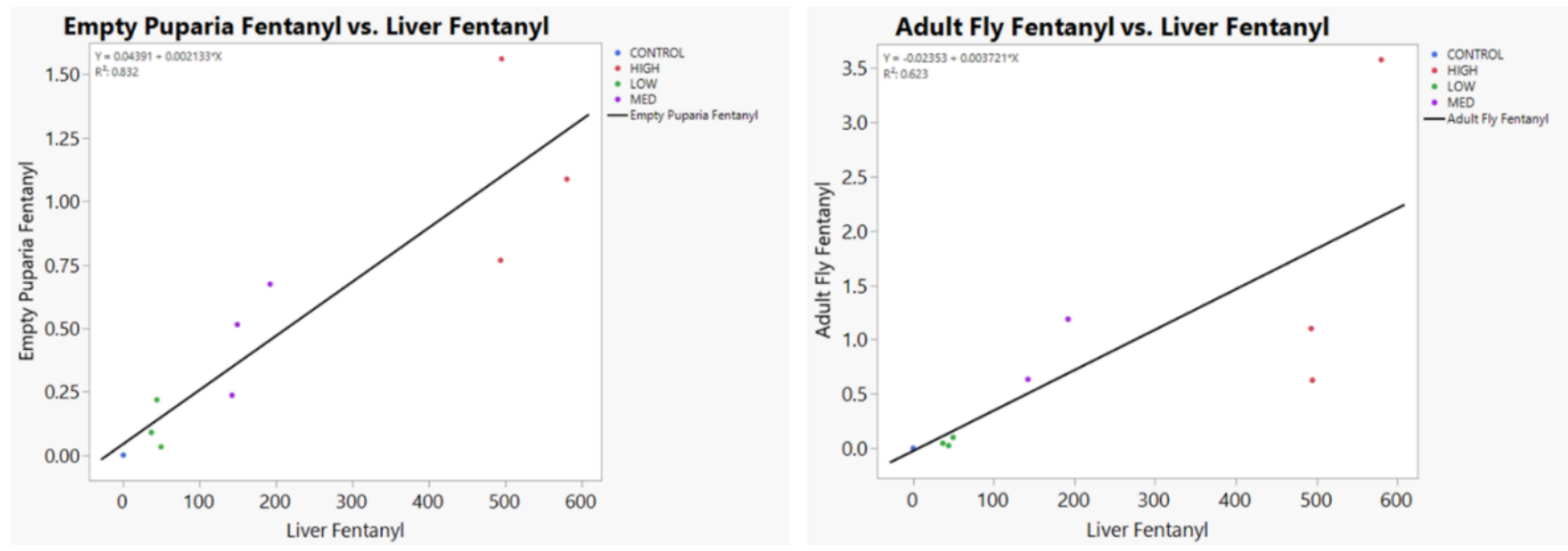

Figure 5.1: Regression plot data for the liver to empty puparia fentanyl concentrations (left) and for liver to adult fentanyl concentrations (right).

Regression plots for the fentanyl concentrations for the insect tissues (empty puparia and adult fly) were created versus the fentanyl concentration of the liver tissue (Figure 5.1). For the empty puparia, the correlation plot generated an $\mathrm{R}^{2}$ value of 0.832 which indicates a moderately strong correlation. For the adult flies the correlation plot generated a lower $R^{2}$ value of 0.623 which is a much weaker correlation. A possible reason for the lower correlation could be created by continued metabolism of fentanyl by the adult insect. The fentanyl concentration for the empty puparia is from only the fentanyl trapped in the chitin of the puparia but the adult concentrations are from the entire insect. 


\subsubsection{Conclusion}

Three extraction techniques were explored for the quantitation of fentanyl and metabolites from empty puparia. The selected extraction technique was the micropulverized QuEChERS extraction and was validated following the ASB 036 (104). Liberation of the drugs from chitin is vital for quantitation when dealing with the low concentrations encountered in insect tissue. Metabolites of norfentanyl and $\beta$ hydroxyfentanyl were quantitated from the insect tissue when the presence of those metabolites were not detected in the liver at the time of egg placement nor at the time of collection which is indicative of insect metabolism. The ability to extract fentanyl and metabolites from late stages of the insect life cycle allows for the insect to be an alternative matrix that persists longer than biological fluids and soft tissues.

Overall, the QuEChERS extraction was successful at extracting fentanyl from empty puparia and adult flies collected from feeding on human liver tissue that contained fentanyl. This provides a method for detecting and quantifying fentanyl and metabolites in an alternate matrix, liver to empty puparia concentrations, liver to adult fly concentrations, and shows the persistence of fentanyl and metabolites through the insect life cycle to adult emergence.

\subsection{Section 2: Morphometric Comparisons of Adult Flies}

\subsubsection{Overview}

This section presents the morphometric comparisons for the adult flies reared in task 2.a. To determine the effects of fentanyl on the growth and development of the blow flies, the mass, length of the wing vein, and length of the tibia of the adult flies were statistically compared using nested ANOVA (task 2.c). Tukey-HSD test will determine which treatments are significantly different to determine if the drug treatments have any significant effects on the insect's development.

Since entomological techniques utilize the growth and development of insects to estimate the minimum time since death, it is crucial to understand the role of fentanyl in the development of Lucilia sericata blow flies. Insects follow a predictable life cycle that xenobiotics can influence. While several 
drugs have been explored in entomotoxicological studies, little is known about the direct effects that fentanyl may pose on the growth and development of blow fly adults.

\subsubsection{Morphometrics}

Much like the larva, the adult flies have physical characteristics that can be measured to evaluate the impact that fentanyl has on the insect's growth. Unfortunately, due to the nature of the fly emerging from the puparia, the empty puparia do not have relevant physical characteristics to record. For the adult fly, mass, the costa wing vein, and tibia of the leg were selected to be measured to explore the impact of the drug on the insect's growth based on literature review (46). Unlike larva, age estimation for adult flies is not a common practice and only morphometrics were used to evaluate the impact of the fentanyl on the adult insects.

\subsubsection{Methods}

\subsubsection{Design}

The insects used in this chapter was the last day of collection (day 21) from the design presented in Methods 4.2.3. Fentanyl was spiked into $200 \mathrm{~g}$ aliquots of human liver homogenate to evaluate four concentrations: control (0 $\mathrm{gg} / \mathrm{kg})$, low $(10 \mu \mathrm{g} / \mathrm{kg})$, medium $(100 \mu \mathrm{g} / \mathrm{kg})$, and high $(350 \mu \mathrm{g} / \mathrm{kg})$ concentrations. For replicate \# 3, an authentic postmortem liver specimen with a fentanyl concentration of $111 \mu \mathrm{g} / \mathrm{kg}$, norfentanyl concentration of $8.1 \mu \mathrm{g} / \mathrm{kg}$, and a 4-ANPP concentration of $5.8 \mu \mathrm{g} / \mathrm{kg}$ was homogenized to be treated as a fifth treatment group. The $200 \mathrm{~g}$ treatment portions were aliquoted into seven $25 \mathrm{~g}$ foil containers (Figure 4.6) with sand as a substrate for pupation. To each aliquot of liver, approximately 70-90 eggs (by mass) were placed and allowed to feed undisturbed until time of collection (Figure 4.6). Empty puparia and adult flies were collected after emergence (day 21) (Figure 4.7). Empty puparia were collected and rinsed in deionized water to remove excess sand substrate followed by a methanol rinse to remove any excess drug from the feeding media. The empty puparia were then allowed to dry before being stored at $-20^{\circ} \mathrm{C}$ with no liquid preservatives added $(7,40,46)$. Collected adult flies were rinsed with deionized water and then methanol before being stored at $-20{ }^{\circ} \mathrm{C}$ with no addition of 
any liquid preservative. No preservative was chosen due to the insect cuticle acting as a semipermeable membrane which can allow water or ethanol to diffuse into the body cavity and potential to leech out the target analytes into the preservative solution (149). Liver samples were collected at the time of egg placement (day 0 ) and at the day of collection to establish the initial tissue concentrations and to evaluate any metabolism of the drugs by the feeding media. This protocol was repeated for three replicates approximately 6-8 weeks apart. The colony was described in section 1 of chapter 4 (4.1.3). For replicate $\# 1$, the colony was at generation 24 , for replicate \#2 the generation was 26 , and for replicate \#3 the generation was 28 .

To evaluate developmental effects, the mass $(\mathrm{mg})$, costa vein length $(\mathrm{mm})$ from wing, and tibia length $(\mathrm{mm})$ of the collected insects were documented (Figure 5.2). Statistical analysis for the physical characteristics including nested ANOVA and Tukey-Kramer HSD were performed using JMP Pro 15.1.0.

\section{$x=0.2 y=2.3 L=2.3 \mathrm{~mm}$}

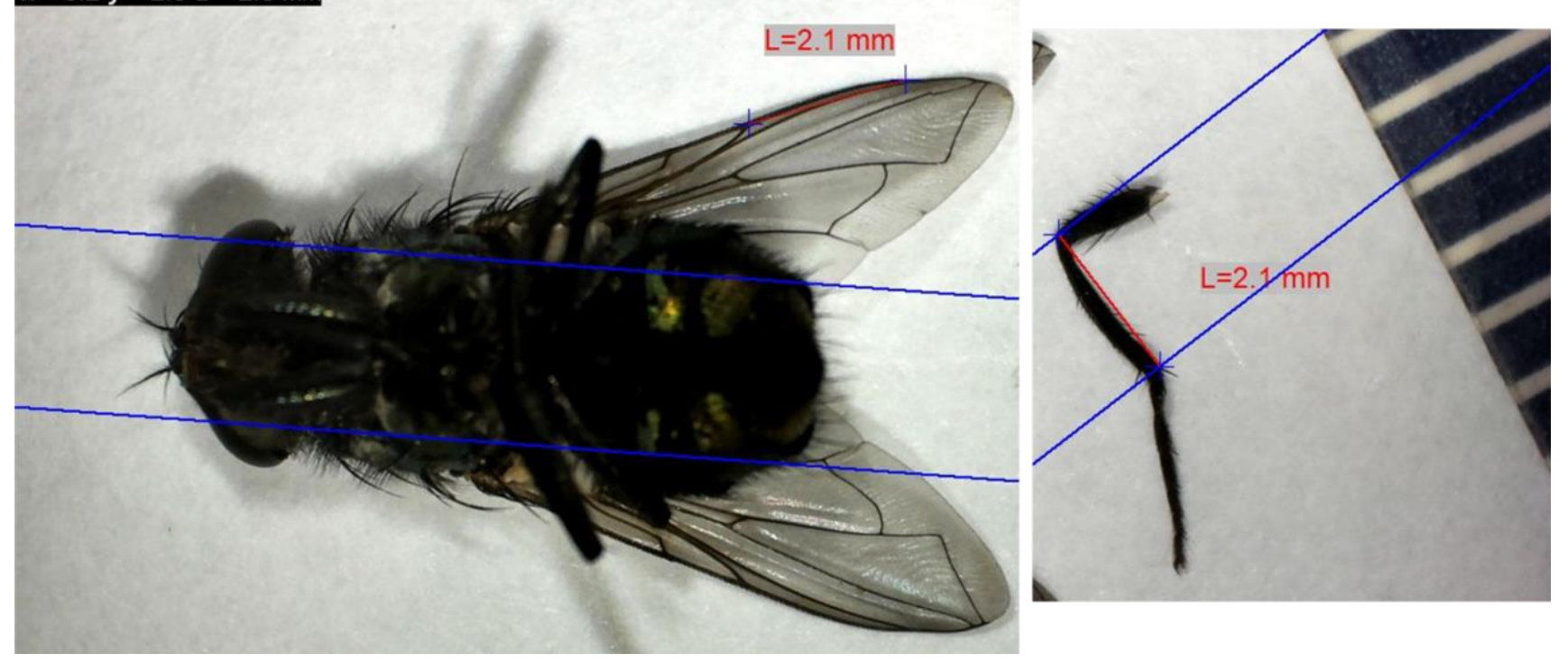

Figure 5.2: Measurements for the costa vein in the fly wing and the tibia length. 


\subsubsection{Results}

For the statistical analysis of the groups, mean was selected as the measure for length, width, and mass of the insects. In the figures below, Tukey-HSD or Steel-Dwase statistical tests were performed with the letters A, B, C, and D representing significant difference. Treatments not connected by a letter are considered significantly different from each other.

\subsubsection{Day 21}

\subsection{Wing Vein}

For day 21 replicate \#1, the medium treatment wing vein was significantly longer than the other three treatment groups $\left(t_{7.9,9.5}=3.19, p=0.0102\right)$ (Figure 5.3). For replicate $\# 2$ and $\# 3$ in terms of wing vein length, there was no significant difference observed between the treatment groups.

Day 21
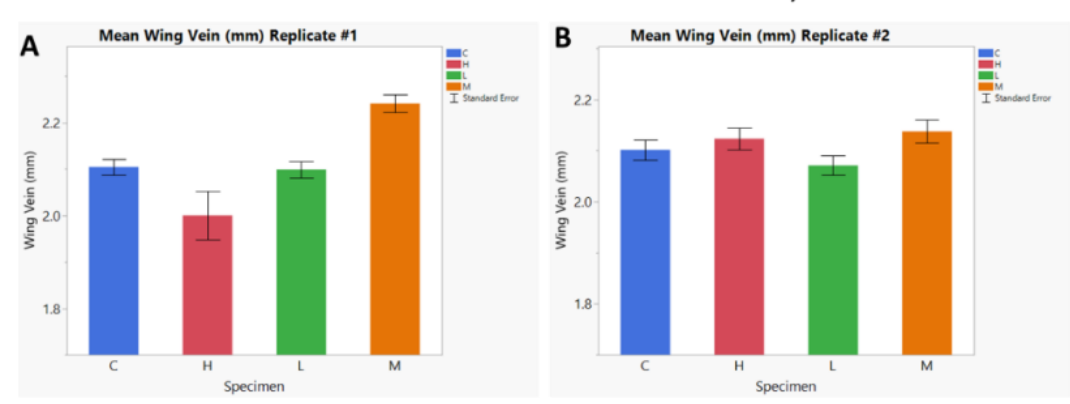

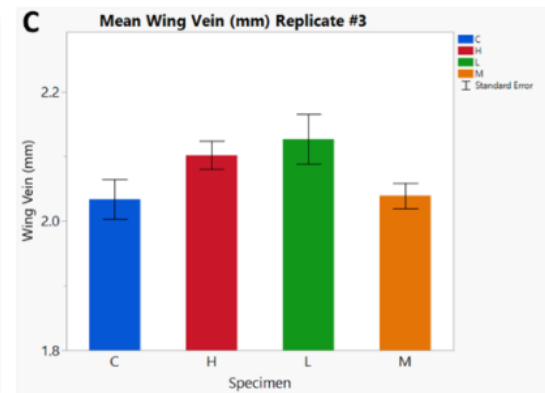

Figure 5.3: Comparison of mean wing vein length for insects collected on day 21 for replicates \#1-3.

\subsection{Tibia Length}

For replicate \#1 tibia length, the medium treatment group was significantly longer than the control and low groups $\left(t_{9.6,11.5}=3.42, p=0.0054\right)$ (Figure 5.4$)$. For replicate \#2 and \#3 in terms of tibia length, there was no significant difference observed between the treatment groups. 
Day 21
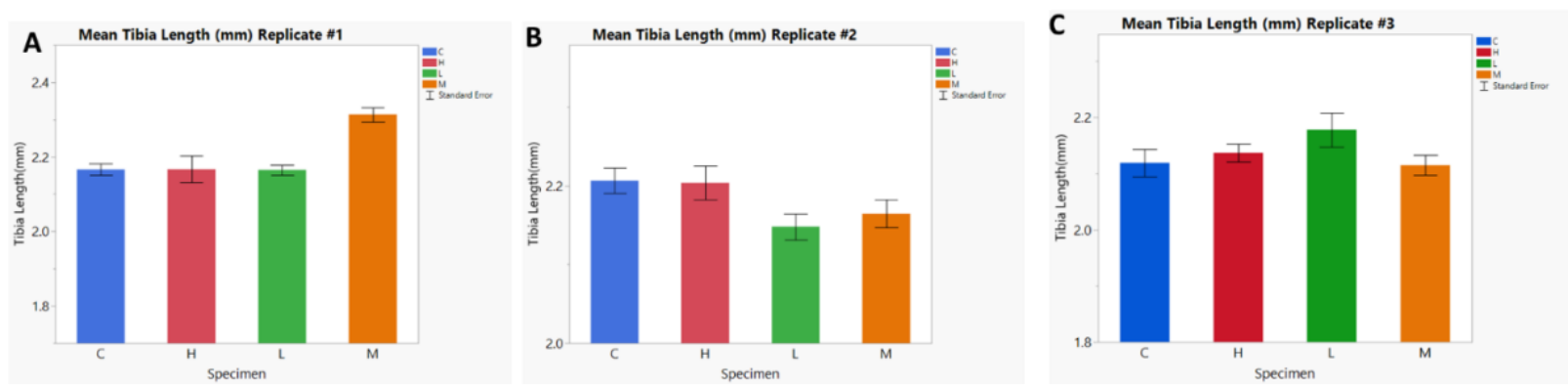

Figure 5.4: Comparison of mean tibia length $(\mathrm{mm})$ for insects collected on day 21 for replicates \#1-3.

\subsection{Mass}

For replicate \#1 in terms of mean mass, the medium treatment group was significantly greater than the control and low groups $\left(t_{9.5,10.6}=3.28, p=0.0076\right)$ (Figure 5.4). For replicate \#2 and \#3 in terms of mass, there was no significant difference observed between the treatment groups.

Day 21
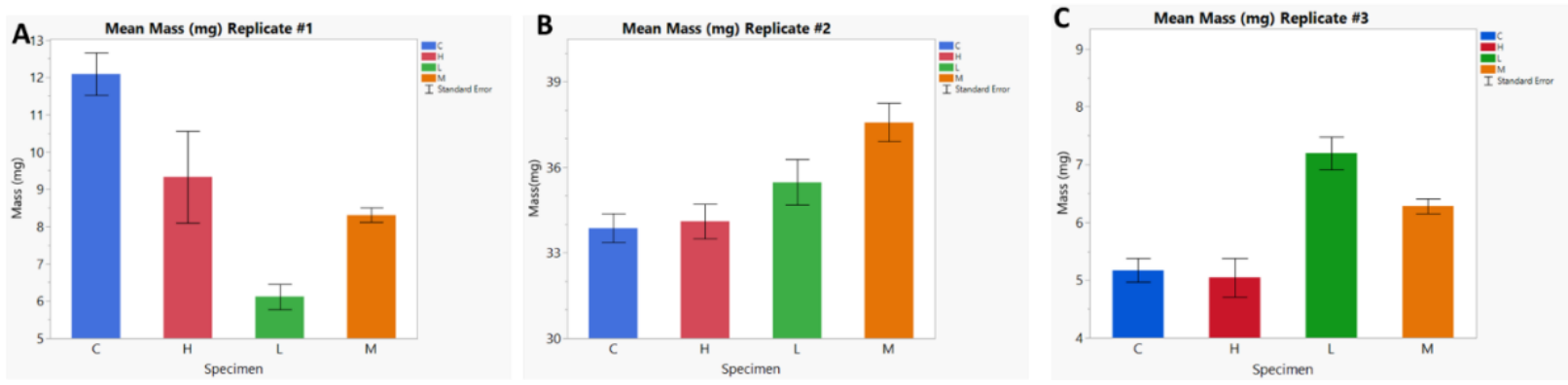

Figure 5.5: Comparison of mean mass for insects collected on day 21 for replicates \#1-3.

\subsubsection{Estimated Survivor Rates}

The estimated survivor rates for the entire study were calculated after the last collection on day 21 (Table 5.9). No significance was observed for the treatment survivor rates but for between the replicates $\left(F_{9,72}=0.948, p=0.4894\right)$, there was a significant decrease in survivor rates in replicate \#2 $\left(F_{2,9}\right.$ $=4.38, p=0.0468)$. 
Table 5.9: Percent estimated survivor rates for all the treatments and replicates combined.

\begin{tabular}{ccc}
\hline & \multicolumn{2}{c}{ Estimated Survivor Rates } \\
\cline { 2 - 3 } Treatment & \% Survived & St Dev \\
\hline Control & 87.4 & 24.9 \\
Low & 92.3 & 26.4 \\
Medium & 79.7 & 22.1 \\
High & 86.6 & 17.7 \\
& & \\
\hline Replicate & \% Survived & St Dev \\
Replicate \#1 & 93.2 & 25.3 \\
Replicate \#2 & 77.1 & 23.7 \\
Replicate \#3 & 84.2 & 14.9 \\
\hline
\end{tabular}

Table 5.10: Gender distribution for the adult flies.

\begin{tabular}{cccccc}
\hline & \multicolumn{2}{c}{ Number of Gender } & & \multicolumn{2}{c}{ \% Gender } \\
\cline { 2 - 3 } \cline { 5 - 6 } Treatment & Male & Females & & \% Male & \% Female \\
\hline Control & 97 & 96 & & 50.3 & 49.7 \\
Low & 81 & 93 & & 46.6 & 53.4 \\
Medium & 56 & 59 & & 48.7 & 51.3 \\
High & 58 & 76 & & 43.3 & 56.7 \\
Authentic & 29 & 26 & & 52.7 & 47.3 \\
\hline
\end{tabular}

\subsubsection{Discussion}

For comparison of adult fly sizes, wing veins have been selected in previously published results (46, 47, 169, 170). Adult Calliphora vomitoria flies were compared by Ireland et al. using the posterior cross vein for the evaluation of overcrowding impacts on insect growth (169). Clark et al. compared the length of the posterior cross vein in adult $L$. sericata flies for the effects of the insects feeding on different body tissues (170). The costa wing vein was chosen for the comparison of adult flies to evaluate the effect of drugs on insect growth $(46,47)$. George et al. compared the length of the costa vein of adult Calliphora stygia for the effects of morphine on the insects (47). Mullany et al used the length of the costa vein of 
adult Calliphora stygia to evaluate the effects of methamphetamine and metabolites (46). George et al. and Mullany et al. also used the tibia length as a metric for the effects of drugs on Calliphora stygia (46, 47).

For the costa wing vein length, only the medium treatment experienced a significant different and no pattern among the treatments was observed. The results for the tibia length were similar to the vein length. Fentanyl did not have a discernable effect on the size of the adults across the treatment groups for the length of the wing vein and tibia. For comparison to previous studies, George et al. did not observe any significant differences in wing vein and tibia length when larva fed on media fortified with morphine (47). Mullany et al. observed increases in the treatment groups for methamphetamine in terms of mass, wing vein, and tibia length (46). The mass of the treatment groups were significantly lower than the control group for all replicates. Even though the length metrics did not decrease the mass of the flies did decrease in the treatment groups with the greatest decrease in the high treatment group. The decrease in mass is similar to the decrease in size of the high treatment for the pupa measurements. The continuation of the effects to the adult flies is reasonable.

\subsubsection{Conclusion}

For the physical characteristics of the adult flies measured to evaluate the impact that fentanyl has on the insect's growth, the only significant difference was a decrease in mass for the treatment groups compared to the control group. There was a significant increase of wing vein and tibia length for the medium treatment group compared to the control but with no significant difference observed in the high treatment a pattern cannot be determined. Unlike larva, age estimation for adult flies is not a common practice and only morphometrics were used to evaluate the impact of the fentanyl on the adult insects. 


\subsection{Section 3: Application of QuEChERS Extraction to Empty Puparia and Adult Flies}

\subsubsection{Overview}

This section presents the toxicological findings for the empty puparia and adult flies for task 1.e. The validated QuEChERS extraction presented in section 1 of this chapter was used for extraction of the feeding media liver and the insect tissue. Presented is the survey of fentanyl and metabolites detected in the empty puparia and adult flies. The later stages of the insect life cycle have advantages over traditional biological matrices as a toxicological matrix due to persistence of the insect during the decomposition process. Due to their slow chemical degradation and proximity to the corpse, pupa and empty puparia can be collected from a cadaver months and even years after death, long after the decomposition process has advanced to skeletonization $(34,163,166)$. The goal of this section is to evaluate the toxicological value of the insect life stages, quantify any metabolites detected for evidence of metabolism by the insect, and to draw any conclusions of the liver tissue concentration to insect tissue concentrations.

\subsubsection{Toxicology Matrix and Correlations}

Detection of drugs in later stages of blowfly development has a benefit over larva because they persist on the cadaver after other suitable toxicology specimens are unavailable (111). When feeding on tissue with drugs present, the drugs are deposited in fat bodies of larva and the chitinized exoskeleton of the pupa (154). The function of the hardened, chitin cuticle of the pupa is to protect the insect from environment during metamorphosis and is resistant to chemical attack, microorganisms, and weathering (44). Due to the structure of chitin, the drugs are often locked in and remain for an extended period of time (34). When pupa emerge to adult flies, the empty puparia remains behind with the drugs trapped in the chitin (44). Pupa and empty puparia, due to the strength of the chitin protected exoskeleton, can persist in the remains long after the soft tissue of the corpse have disappeared (34). This allows for toxicological analysis to take place on the pupa and empty puparia even in advanced decomposition or skeletonized remains when traditional toxicology specimens like blood, urine and organ tissue are not available for testing. The use of empty puparium for toxicological analysis has utility due to their ability to 
exist for extend periods on remains, even after skeletonization of the corpse (171). Miller et al. were able to isolate amitriptyline and nortriptyline from empty fly puparia and beetle exuvia recovered from mummified human remains in an indoor decomposition case (163). From stomach contents and analysis of the desiccated brain, multiple drug intoxication, including amitriptyline, was identified as the cause of death (163). Puparia have been discovered in Egyptian and pre-Columbia Peruvian mummies which displays the ability of this type of evidence to remain in the environment for hundreds of years (172).The ability of empty puparia to remain in soil surrounding the remains for hundreds of years, makes empty puparia a prime target specimen for toxicology analysis in cadavers discovered in the late stage of decomposition (163).

\subsubsection{Methods}

The samples from the design in methods 4.2.3 were used for the toxicology analysis of the collected insects. Insects from collection day 21 were selected for toxicology analysis after the imaging and morphometric characteristic measurements (Figure 4.7). The insects were pooled $(n=16)$ as empty puparia or as adult flies and extracted using the validated QuEChERS extraction. Liver samples were collected at the time of egg placement (day 0 ) and at the day of collection to establish the initial tissue concentrations and to evaluate any metabolism of the drugs by the feeding media. A survey of the persistence and prevalence of fentanyl and metabolites was created.

\subsubsection{Results}

The previously discussed validated QuEChERS extraction was applied to the empty puparia and adult flies collected on day 21 for all four treatments over three replicates with one additional treatment set of an authentic postmortem liver specimen containing fentanyl (Table 5.11). The insects were analyzed as pooled samples $(n=16)$ in duplicate for all three replicates $(n=6)$. 
Table 5.11: Empty puparia and adult drug concentrations from day 21 collections for all three replicates.

\begin{tabular}{|c|c|c|c|c|c|}
\hline \multirow{3}{*}{ Compound } & \multicolumn{5}{|c|}{ Empty Puparia } \\
\hline & \multicolumn{5}{|c|}{ Pooled Empty Puparia $(\mu \mathrm{g} / \mathrm{kg})$} \\
\hline & Control & Low & Medium & High & Authentic \\
\hline 4-ANPP & ND & ND & ND & ND & ND \\
\hline$\beta$-Hydroxyfentanyl & ND & $2.0 \pm 0.4$ & $9.0 \pm 0.1$ & $5.1 \pm 2.2$ & ND \\
\hline Fentanyl & ND & $<$ LLOQ & $0.4 \pm 0.2$ & $2.4 \pm 1.6$ & $3.4 \pm 1.1$ \\
\hline \multirow[t]{2}{*}{ Norfentanyl } & ND & ND & $1.2 \pm 0.6$ & $2.5 \pm 0.7$ & $1.6 \pm 0.2$ \\
\hline & \multicolumn{5}{|c|}{ Adult Flies } \\
\hline \multirow{2}{*}{ Compound } & \multicolumn{5}{|c|}{ Pooled Adult Flies $(\mu \mathrm{g} / \mathrm{kg})$} \\
\hline & Control & Low & Medium & High & Authentic \\
\hline 4-ANPP & ND & ND & ND & ND & ND \\
\hline$\beta$-Hydroxyfentanyl & ND & ND & ND & ND & ND \\
\hline Fentanyl & ND & $1.1 \pm 0.7$ & $1.8 \pm 1.7$ & $3.5 \pm 3.5$ & $0.2 \pm 0.2$ \\
\hline Norfentanyl & ND & $1.1 \pm 0.4$ & $2.4 \pm 1.5$ & $2.2 \pm 0.9$ & $<$ LLOQ \\
\hline
\end{tabular}

For the empty puparia and adult flies collected on day 21 , fentanyl was detected in all treatments across all three replicates but was below the LLOQ for the low treatment in the empty puparia (Table 5.9). For the adult flies a trend emerged. As the concentration of the fortified liver treatment increased, the concentration of fentanyl observed in the adult fly extractions also increased. For the empty puparia collected from the low treatment, the average $\beta$-hydroxyfentanyl concentration $(n=4)$ across the three replicates was $2.0 \pm 0.4 \mu \mathrm{g} / \mathrm{kg}$, fentanyl was detected but below LLOQ, norfentanyl, and 4-ANPP were not detected. For the empty puparia collected from the medium treatment, the average $\beta$-hydroxyfentanyl concentration $(n=2)$ across the three replicates was $9.0 \pm 0.1 \mu \mathrm{g} / \mathrm{kg}$, fentanyl $(\mathrm{n}=6)$ was $0.4 \pm 0.2 \mu \mathrm{g} / \mathrm{kg}$, norfentanyl $(\mathrm{n}=4) 1.2 \pm 0.6 \mu \mathrm{g} / \mathrm{kg}$, and 4-ANPP was not detected. For the high treatment, the average $\beta$ hydroxyfentanyl concentration $(n=4)$ across the three replicates was $5.1 \pm 2.2 \mu \mathrm{g} / \mathrm{kg}$, fentanyl $(\mathrm{n}=6)$ was $2.4 \pm 1.6 \mu \mathrm{g} / \mathrm{kg}$, norfentanyl $(\mathrm{n}=4) 2.5 \pm 0.7 \mu \mathrm{g} / \mathrm{kg}$, and 4-ANPP was not detected. For the authentic 
treatment, the fentanyl average concentration $(n=2)$ was $3.4 \pm 1.1 \mu \mathrm{g} / \mathrm{kg}$, norfentanyl $(\mathrm{n}=2) 1.6 \pm 0.2$

$\mu \mathrm{g} / \mathrm{kg}, \beta$-hydroxyfentanyl, and 4-ANPP was not detected. Box plots were created for these averages from fentanyl (Figure 5.6), norfentanyl (Figure 5.7), and $\beta$-hydroxyfentanyl (Figure 5.8).

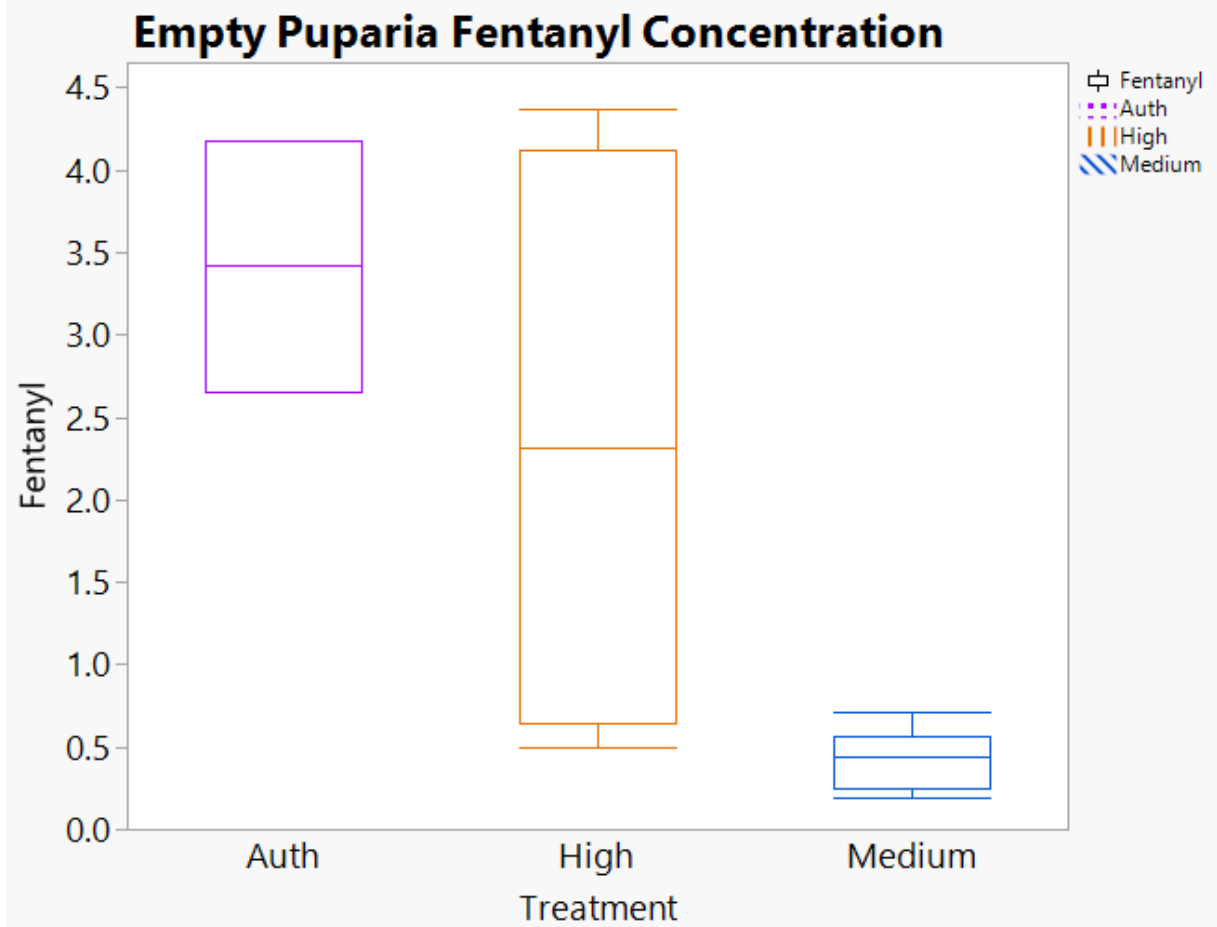

Figure 5.6: Box plot for the concentration of fentanyl quantitated for the pooled empty puparia specimens collected from the authentic, medium, and high treatments on day 21 for all three replicates $(n=6)$. 


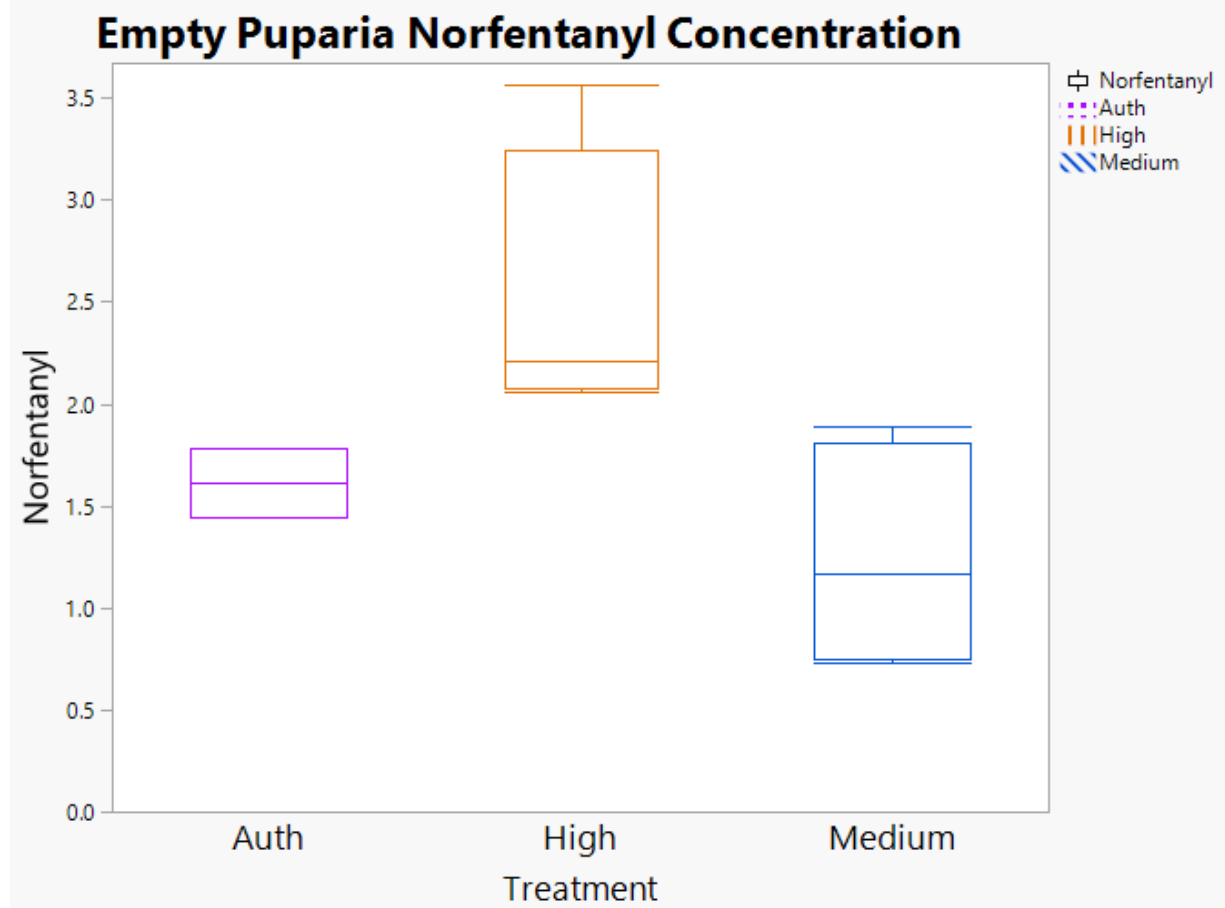

Figure 5.7: Box plot for the concentration of norfentanyl quantitated for the pooled empty puparia specimens collected from the authentic, medium, and high treatments on day 21 for all three replicates $(n=6)$.

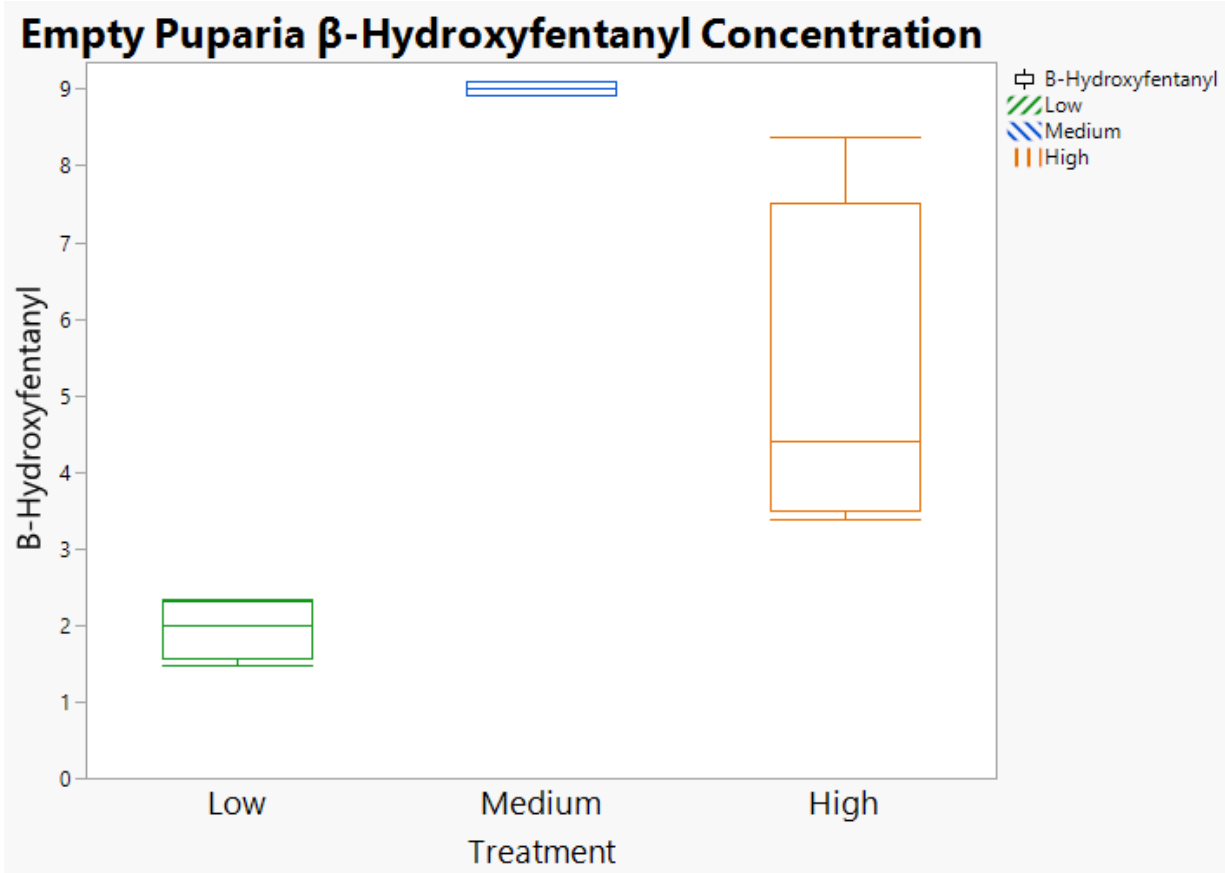

Figure 5.8: Box plot for the concentration of $\beta$-hydroxyfentanyl quantitated for the pooled empty puparia specimens collected from the low, medium, and high treatments on day 21 for all three replicates $(n=6)$. 
For the adult flies collected from the low treatment, the average norfentanyl concentration $(n=2)$ across the three replicates was $1.1 \pm 0.4 \mu \mathrm{g} / \mathrm{kg}$, fentanyl ( $\mathrm{n}=2)$ was $1.1 \pm 0.7 \mu \mathrm{g} / \mathrm{kg}$, $\beta$-hydroxyfentanyl, and 4-ANPP were not detected. For the adult flies collected from the medium treatment, the average fentanyl concentration ( $\mathrm{n}=4)$ was $1.8 \pm 1.7 \mu \mathrm{g} / \mathrm{kg}$ and norfentanyl $(\mathrm{n}=4)$ was $2.4 \pm 1.5 \mu \mathrm{g} / \mathrm{kg}$. For the high treatment, the average fentanyl concentration $(n=4)$ across the three replicates was $3.5 \pm 3.3 \mu \mathrm{g} / \mathrm{kg}$ and norfentanyl $(n=4) 2.2 \pm 0.9 \mu \mathrm{g} / \mathrm{kg}$. For the authentic treatment, the fentanyl average concentration $(n=3)$ was $0.2 \pm 0.2$ $\mu \mathrm{g} / \mathrm{kg}$, norfentanyl ( $\mathrm{n}=3$ ) was detected below the LLOQ, $\beta$-hydroxyfentanyl, and 4-ANPP were not detected. Box plots were created for these averages from fentanyl (Figure 5.9) and norfentanyl (Figure 5.10). The metabolites $\beta$-hydroxyfentanyl and 4-ANPP were not detected in the adults flies in any of the extracted specimens across all treatments.

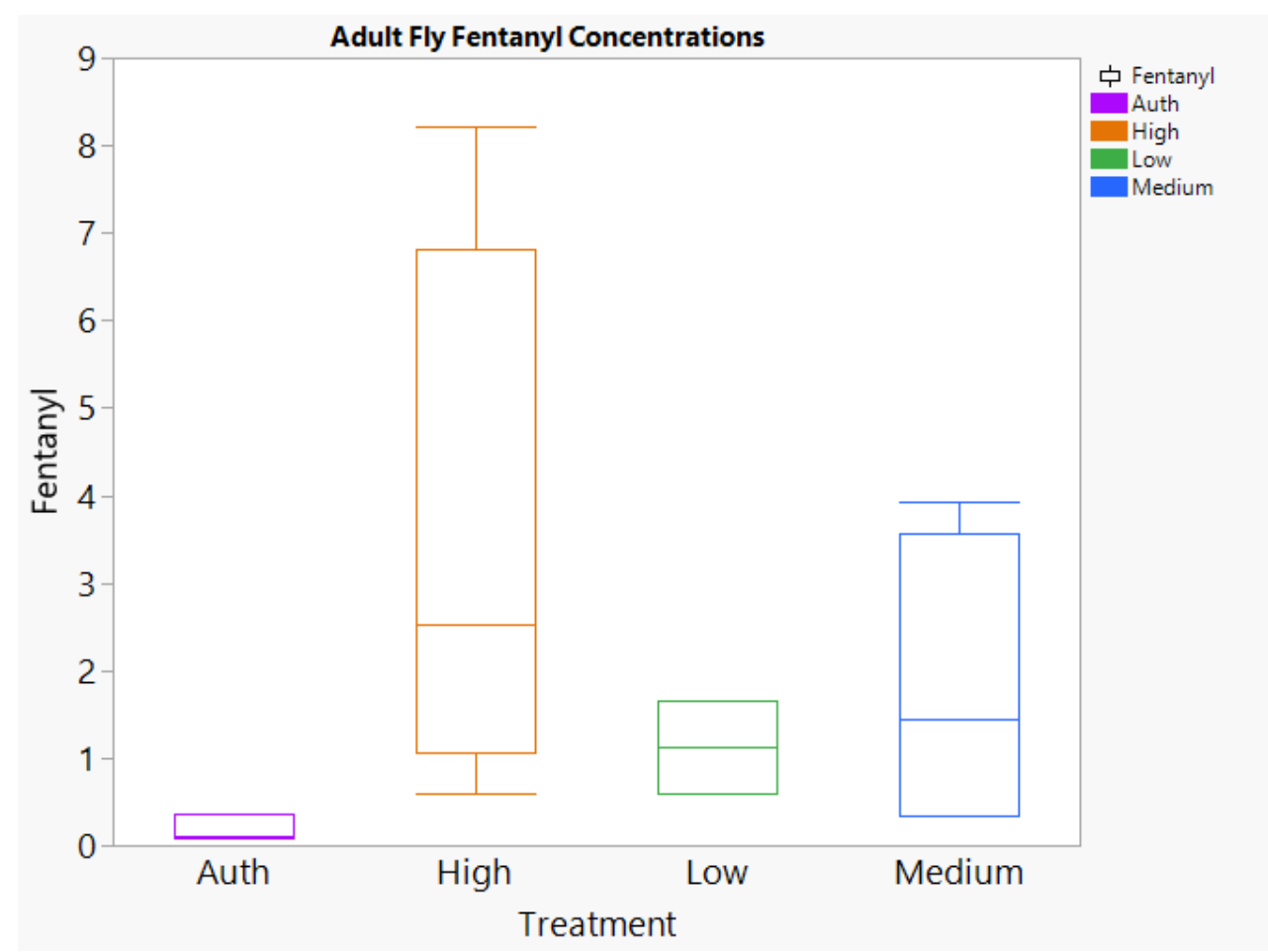

Figure 5.9: Box plot for the concentration of fentanyl quantitated for the pooled adult fly specimens collected from the low, medium, and high treatments on day 21 for all three replicates $(n=6)$. 


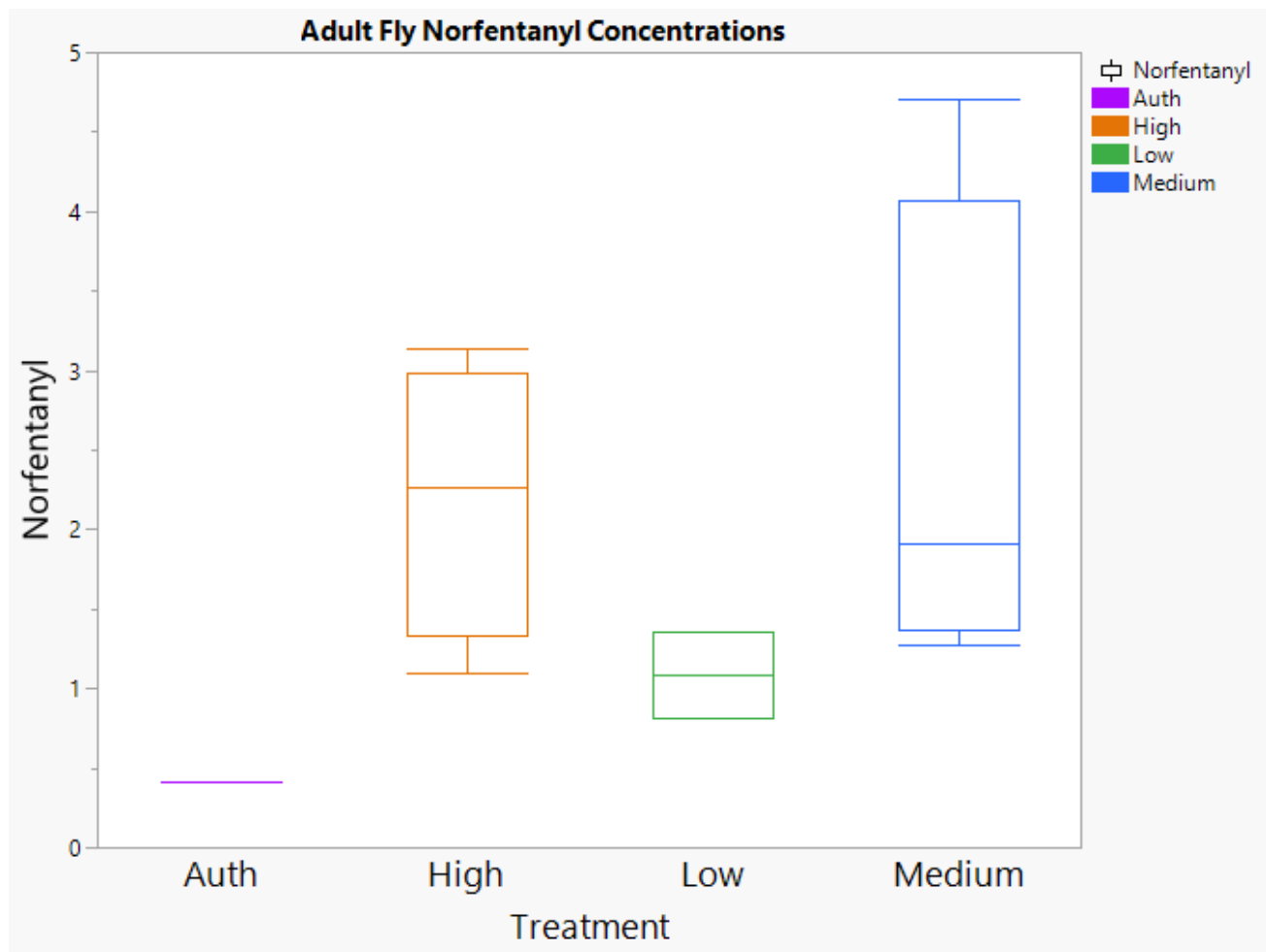

Figure 5.10: Box plot for the concentration of norfentanyl quantitated for the pooled adult fly specimens collected from the low, medium, and high treatments on day 21 for all three replicates $(n=6)$.

\subsubsection{Conclusion}

Fentanyl and metabolites were extracted from the empty puparia and adult flies feeding on liver tissue fortified with fentanyl. $\beta$-hydroxyfentanyl was detected in the empty puparia but not in the adult flies. $\beta$-hydroxyfentanyl was detected in the later larval and pupal stages therefore the persistence of the metabolite to the empty puparia is expected but the lack of detected it the adult flies indicates either the compound is sequestered by the chitin of the pupa and left behind in the exuviate or the adult flies could be continuing to excrete and clear the compound from their system. The detection of fentanyl and norfentanyl from the empty puparia and the adult flies exemplify the utility of this matrix as a biomarker for the presence of fentanyl in the tissues the insects fed on which can be a proxy to postmortem tissue drug presence. The detection of fentanyl and norfentanyl from the empty puparia and the adult flies also indicates that a portion of the drug is left behind in the fly exuviate but also persists to the adult fly. 
These findings strengthen the utility of empty puparia for the detection of fentanyl and metabolites collected from tissues fortified with fentanyl. This is promising for the use of late stage insect tissue for the detection of postmortem tissue drug presence. 
Chapter 6: General Conclusions 


\subsection{Chapter 3: Development and Application of QuEChERS Extraction Method for the Quantitation of Fentanyl and Metabolites from Liver Tissue}

The ability to extract a drug or toxin from a matrix and the instrumental analysis are the main components of toxicology analysis. For the analysis of complex biological matrices, three extractions were evaluated: liquid-liquid extraction (LLE), solid-phase extraction (SPE), and a QuEChERS extraction. For each extraction technique, matrix effects, recovery, and process efficiency were evaluated. The QuEChERS extraction outperformed the SPE and LLE techniques and was selected for validation and evaluation of authentic specimens. The QuEChERS technique had recoveries higher than $90.2 \%$, matrix effects greater than $80.4 \%$, and process efficiencies greater than $76.7 \%$ with $\% \mathrm{CV}$ values below $12.8 \%$ for fentanyl, norfentanyl, and 4-ANPP. The bias for all three target analytes at all three concentrations did not exceed $\pm 20 \%$ with repeatability and reproducibility within the $\pm 20 \%$ threshold and the analytical measurable range (AMR) of $0.4-80 \mu \mathrm{g} / \mathrm{kg}$ for 4-ANPP and $0.5-100 \mu \mathrm{g} / \mathrm{kg}$ for fentanyl and norfentanyl. The fentanyl concentrations reported ranged from 56.6 to $462.3 \mu \mathrm{g} / \mathrm{kg}$ with a mean of $149.2 \mu \mathrm{g} / \mathrm{kg}(\mathrm{n}=10)$ for authentic postmortem liver specimens. Overall, the QuEChERS technique met all the acceptable criteria and was shown to be effective at extracting and quantitating fentanyl, norfentanyl, and 4-ANPP. The advantages of the QuEChERS extraction include the direct extraction of the tissues, a cleaner sample to reduce ionization enhancement or suppression, elimination of cross contamination by eliminating the need for bladed homogenizers, and provided a reduction of extraction mass.

The QuEChERS extraction was expanded to 34 analytes including fentanyl, metabolites, and fentanyl analogs, validated following the American Academy of Forensic Sciences Standards Board (ASB) standard 036 requirements, and the protocol was applied to 22 authentic postmortem liver specimens. The bias for all 34 target analytes at low, medium, and high concentrations did not exceed $\pm 20 \%$ with repeatability and reproducibility within the $20 \%$ threshold. The QuEChERS technique had recoveries greater than $93.3 \%$, matrix effects greater than $76.8 \%$, and process efficiencies greater than $81.5 \%$ for all 34 target analytes. Of the 22 authentic postmortem samples, 17 were multidrug positive with at least one 
fentanyl analog and fentanyl present indicating a prevalence of multiple fentanyl types in use. Nine of the 22 samples contained at least two fentanyl analogs plus fentanyl and metabolites. Three of the 22 samples contained three fentanyl analogs plus fentanyl and metabolites.

Overall, the QuEChERS extractions met all the acceptable criteria set by the ASB Standard 036 requirements for liver extraction and proved to be effective at extracting and quantitating fentanyl as well as fentanyl analogs.

\subsection{Chapter 4: Evaluation of the Effects of Fentanyl on Blowfly Larva and Application of QuEChERS Extraction Method for the Quantitation of Fentanyl and Metabolites from Larva and Pupa (Lucilia sericata)}

The overarching goals of this study was to evaluate the developmental effects of fentanyl on immature stages of the blow fly Lucilia sericata (Meigen) and to monitor the prevalence of fentanyl and metabolites from larval and pupal tissue after feeding on liver tissue with the presence of fentanyl. The QuEChERS extraction method developed for postmortem liver was adapted for extraction of larval and pupal tissue. The extraction protocol met all the criteria mapped out by the ASB and was applied to authentic insect samples that were reared on human tissue containing fentanyl.

This study presented morphometric measures of length, width, and mass for the insects collected; however, larval length was the most significant physical characteristic. In comparison of individual collection days, at least one of the treatment groups (low, medium, or high) was significantly different (nested ANOVA, $\mathrm{p}<0.05$ ) from the control group over all three replicates. The low, medium, and high treatment groups reached a greater maximum length compared to the control group in most of the replicates indicating fentanyl had some effect on the length of the insects. Using a polynomial equation on the growth curve of the control group, the estimation of age of the insects showed that there was often an increase in growth at the early life stages, followed by a decrease in the later larval stages. The age estimation for the high treatment group was as high as $1.4 \mathrm{~d}$ younger than the actual age and for the authentic set as high as $-2.9 \mathrm{~d}$ younger than the actual age. Every treatment group had an underestimation 
in age of $1 \mathrm{~d}$ in at least one day of collection among the three replicates. With the impact of fentanyl on the length of the larval length, it can be concluded that fentanyl can reduce the larval growth by at least 1 day and up to 3 days. This indicates age estimation based on length can be impacted by the presence of fentanyl in the feeding media and needs to be taken into consideration when estimating ${ }_{\min } \mathrm{PMI}$.

The validated QuEChERS extraction was applied to the larva and pupa collected on days 3, 6, and 10 for all four treatments over three replicates with one additional treatment set of an authentic postmortem liver specimen containing fentanyl. Fentanyl was detected in the larvae and pupae that were collected from the human liver tissue. Fentanyl was quantitated in all larva collected on day three from all three treatments of low, medium, and high across the three replicates. By quantitating fentanyl in all three treatments, a correlation plot and simple linear regression was performed using the known concentrations of the fortified liver feeding substrate. Strong correlation of larva concentration to liver tissue concentration was observed for all replicates in the single and pooled larva specimens. The single larva to liver correlations observed an $\mathrm{R}^{2}$ value ranging from 0.908 to 0.937 and the pooled larva to liver correlations observed an $\mathrm{R}^{2}$ value ranging from 0.959 to 0.988 . To further investigate the utility of the correlation, the larva collected from the authentic treatment group was used to test the ability of the linear regression to estimate the liver fentanyl concentration using the larva fentanyl concentration. After estimation, the single larva fentanyl extractions had an average bias $(n=5)$ of -21.7 and the pooled specimens had an average bias $(n=5)$ of -32.1 . After dropping an outlier, the average bias $(n=4)$ for the single larva was -11.8 . It is not known what created the outlier, but this highlights why it is important to analyze multiple replicates for insect toxicology analysis or to sample a pooled specimen. Metabolites of norfentanyl and $\beta$-hydroxyfentanyl were quantitated from the insect tissue when the presence of those metabolites were not detected in the liver at the time of egg placement nor at the time of collection. It can be derived that the most likely creation of these metabolites was metabolism of fentanyl by the insect to these compounds. Also, the metabolite 4-ANPP was quantitated from the feeding larva insects 
collected from the authentic liver specimen; however, this liver had a quantified 4-ANPP prior to egg placement and with no other insect containing a quantifiable concentration of 4-ANPP, no metabolism of fentanyl to 4-ANPP by the insects can be determined.

Overall, the QuEChERS extraction was successful at extracting fentanyl from larvae and pupae collected from feeding on human liver tissue that contained fentanyl. This provides a method for detecting and quantifying fentanyl in an alternate matrix, liver to larvae concentrations, and shows the persistence of fentanyl through the insect life cycle to pupation.

\subsection{Chapter 5: Evaluation of the Effects of Fentanyl on Blowfly Adults and Application of QuEChERS Extraction Method for the Quantitation of Fentanyl and Metabolites from Empty Puparium and Adult Flies (Lucilia sericata)}

Three extraction techniques, micropulverized QuEChERS extraction, acidic digestion QuEChERS

extraction and the basic digestion LLE were tested for the extraction of fentanyl and metabolites from the empty puparia. The extraction that performed the best was selected for validation. To evaluate the extraction techniques, ionization suppression/enhancement, recovery, process efficiency, and the ability to extract from an authentic set of empty puparia collected from insects fed on liver tissue fortified with fentanyl were compared for the three techniques. The micropulverization QuEChERS extraction method was selected and validated for the analysis of fentanyl and metabolites from empty puparia and adult fly tissue.

Much like the larva, the adult flies have physical characteristics that can be measured to evaluate the impact that fentanyl has on the insect's growth. Unfortunately, due to the nature of the fly emerging from the puparia, the empty puparia do not have relevant physical characteristics to record. Morphometric comparisons for the collected adult flies were recorded to evaluate the effects each treatment had on growth. To determine the effects of fentanyl on the growth and development of the blow flies, the mass, costa wing vein, and tibia length were statistically compared. Unlike larva, age estimation for adult flies is not a common practice and only morphometrics were used to evaluate the 
impact of the fentanyl on the adult insects. For the physical characteristics of the adult flies measured to evaluate the impact that fentanyl has on the insect's growth, the only significant difference was a decrease in mass for the treatment groups compared to the control group. There was a significant increase of wing vein and tibia length for the medium treatment group compared to the control but with no significant difference observed in the high treatment a pattern cannot be determined.

Detection of drugs in later stages of blowfly development has a benefit over larva because they persist on the cadaver after other suitable toxicology specimens are unavailable. Fentanyl and metabolites were extracted from the empty puparia and adult flies feeding on liver tissue fortified with fentanyl. The survey of fentanyl and metabolites detected in the empty puparia and adult flies included the detection of fentanyl, $\beta$-hydroxyfentanyl, and norfentanyl. The metabolite 4-ANPP was not detected in any empty puparia or adult fly specimens after being detected in the authentic larva specimens. $\beta$-hydroxyfentanyl was detected in the empty puparia but not in the adult flies. $\beta$-hydroxyfentanyl was detected in the later larval and pupal stages therefore the persistence of the metabolite to the empty puparia is expected but the lack of detected it the adult flies indicates either the compound is sequestered by the chitin of the pupa and left behind in the exuviate or the adult flies could be continuing to excrete and clear the compound from their system. The detection of fentanyl and norfentanyl from the empty puparia and the adult flies exemplify the utility of this matrix as a biomarker for the presence of fentanyl in the tissues the insects fed on which can be a proxy to postmortem tissue drug presence. The detection of fentanyl and norfentanyl from the empty puparia and the adult flies also indicates that a portion of the drug is left behind in the fly exuviate but also persists to the adult fly.

These findings strengthen the utility of empty puparia for the detection of fentanyl and metabolites collected from tissues fortified with fentanyl. This is promising for the use of later stage insect tissue for the detection of postmortem tissue drug presence. 
Overall, the goals of detecting fentanyl and metabolites from all life stages of the insects and the observation of any effects of fentanyl on the growth of the insects was accomplished. For the growth and developmental impacts of fentanyl on the insects, even though a statistical trend for the impact of the drug was not observed, the variations in the insect growth should be taken into consideration when estimating $\min _{\mathrm{PMI}} \mathrm{Phen}$ fentanyl is present in the cadaver. Fentanyl was observed in all insect life stages (feeding larva, wandering larva, pupa, empty puparia, and adult flies) for the insects feeding on tissue with fentanyl present. This indicates the insects do incorporate the drugs into their tissues to some degree and fentanyl can be detected in the insect tissue collected from cadavers that contain fentanyl. From the later stages of wandering larva, pupa, and empty puparia, fentanyl concentrations decreased and fentanyl metabolites were observed more frequently. This is evidence of the insect's (Lucilia sericata) ability to metabolize and eliminate fentanyl from their bodies and with greater success in the later or non-feeding life stages. In regard to the metabolites observed, norfentanyl was the only metabolite detected in the early life stages (feeding larva) while $\beta$-hydroxyfentanyl in combination with norfentanyl was detected in the later life stages (wandering larva, pupa, and empty puparia). The significance of these findings could be a change in the metabolic pathways or of the later life stage insects or more likely the accumulation of $\beta$-hydroxyfentanyl in the insect tissue as the insect metabolizes fentanyl without the insect being able to eliminate the metabolite from their tissue. The increase in the frequency of the detection of $\beta$ hydroxyfentanyl could be an indication of the metabolite being trapped in the insect tissue and accumulating in the later insect life stages. Another unique observation is the absence of $\beta$ hydroxyfentanyl in the adult flies while the metabolite was detected in the empty puparia that the adult flies emerged from. These findings could be indicative of sequestering of $\beta$-hydroxyfentanyl into the exoskeleton of the pupa and therefore trapped in the empty puparia that is left behind when the adult insect emerges. Another possible explanation could be the elimination of $\beta$-hydroxyfentanyl by the adult fly in the meconium that is defecated after emergence. Detection of fentanyl and metabolites in the 
empty puparia is of great importance as the rugged chitinized exoskeleton can persist in the environment for hundreds of years and depending on the stability of the drug, this evidence can be utilized long after soft tissues or fluids are no longer available. 


\section{Future Work}

This work consisted of large data set and provides valuable information for the impact of fentanyl on the growth of Lucilia sericata. The focus of this study was to observe the effects of fentanyl on the insects and to evaluate the ability to extract fentanyl and metabolites from all the insect life stages. Even though the work presented is valuable, there are still limitations to the study. One limitation was the days collected, due to limited resources the number of collection days were chosen to optimize the amount of toxicology data gathered. Days there were selected in the late stage of the life cycle were not sensitive to changes in the development time of the flies in order to ensure the toxicology specimens were available however this reduced the value of data for development impact. In order to have toxicology samples and maximize resources, later life stages were chosen for collection at days that had a higher chance of having the majority of the insects in the desired life stage. This reduced the efficacy of detecting subtle impacts that fentanyl could have on the insects while still being able to observe if the drug had major effects on the insects. More data collected at the later life stages could provide a better look at the percent stage of insects in the later life cycle. A related limitation is no information was collected between day 6 and day 10 for the life cycle of the insects. Larva were still observed with the pupa at day 10 collection times, while more larva were collected in the treatment groups but the difference was not significant. More replicates and/or the addition of collection days between day 6 and day 10 could identify if there are significant impacts on the insect as they transition to pupa. Based on the larval growth patterns, the treatment groups either grew to their maximum length or grew to a length longer than the treatment groups at some stage of their life cycle however, the as the larval stage continue the slopes of the growth curves slowed. This could 
be an indication of a slowdown to pupation and the collection days between 7 and 9 could provide more insight to this effect.

Some future work that I would recommend to enhance the data collected would be to expand and separate the experimental design. The design I used in this study was focused on maximizing the current resources on hand while gathering the most observable data for both the effects of the drug on the insects and to provide a toxicological analysis on the collected samples. With the conclusions discussed above, further study with experimental designs focused entirely on either the developmental effects of fentanyl on the insect and the toxicological analysis can be implemented.

For the toxicology future work, this study provided regression plots that displayed high correlation of the liver tissue fentanyl concentration to feeding larvae fentanyl concentrations. This is an important step in discovering correlation of insect and tissue concentrations, but this does not mean the concentrations will correlate in authentic case work. Future work should include more replication and additional tissues for fortification like muscle and heart tissue. This work and previous literature have shown that the later life stages of insect varies as metabolism and ability of the insect to excrete the drug creates variations. This would be the next step to correlating real world scenarios.

For developmental effects, an increase in replicates and an increase in collection days would be my recommendation. Growth rates of insects can vary, and more replicates can be useful in identifying variation created by genetics compared to treatment. Also increasing the collection days to include up to pupation and beyond. For this study there was a gap in between day 6 and day 10 due to resources but collection days 7 through 9 could have valuable insight to 
the stage development of the insects. Also, days 7 through 9 should be more discriminatory to the transition of larva to pupa as day 10 was chosen in this study to improve the likelihood of observing enough pupa for toxicology analysis but sacrificed observations of percent stage. The same is true for emergence, more studies can be applied to see the effect the fentanyl has on the emergence of the adult flies by collecting at more days between day 10 and day 21 .

This study provided insight into the effect of fentanyl on Lucilia sericata and the detection of fentanyl and metabolites from all of the insect's life stages to lay the groundwork for future studies. 


\section{References}

1. Usui, K., Hashiyada, M., Hayashizaki, Y., Igari, Y., Hosoya, T., Sakai, J., et al. (2014) Application of modified QuEChERS method to liver samples for forensic toxicological analysis. Forensic Toxicology, 32, 139-147.

2. Harvey, M., Gasz, N. and Voss, S. (2016) Entomology-based methods for estimation of postmortem interval. Research and Reports in Forensic Medical Science, 2016: 10.2147/rrfms.s68867.

3. Introna, F., Altamura, B.M., Dell'Erba, A. and Dattoli, V. (1989) Time since death definition by experimental reproduction of Lucilia sericata cycles in growth cabinet. Journal of Forensic Sciences, 34, $12661 \mathrm{~J}$.

4. Cox, J., Train, A., Field, A., Ott, C., DelTondo, J., Kraner, J., et al. (2020) Quantitation of fentanyl and metabolites from liver tissue using a validated QuEChERS extraction and LC-MS-MS analysis. Journal of Analytical Toxicology, 44, 957-967.

5. Cox, J., Mathison, K., Ott, C., DelTondo, J., Kraner, J.C., DeCaprio, A.P., et al. (2021) Quantitation and Validation of 34 fentanyl analogs from liver tissue using a QuEChERS extraction and LC-MS-MS analysis. Journal of Analytical Toxicology, 2021: 10.1093/jat/bkab009.

6. Morris, B. and Dadour, R. (2014) Essentials of Autopsy Practice. Rutty, G.N. (ed), Springer-Verlag London.

7. Amendt, J., Campobasso, C.P., Gaudry, E., Reiter, C., LeBlanc, H.N. and J. R. Hall, M. (2007) Best practice in forensic entomology - Standards and guidelines. International Journal of Legal Medicine, $121,90-104$.

8. Tarone, A.M. and Foran, D.R. (2006) Components of developmental plasticity in a Michigan population of Lucilia sericata (Diptera: Calliphoridae). Journal of Medical Entomology, 43, 1023-33. http://www.ncbi.nlm.nih.gov/pubmed/17017243.

9. Higley, L.G., Haskell, N. and Roe, A. (2014) The author ( s ) shown below used Federal funds provided by the U.S. Department of Justice and prepared the following final report : Document Title : Establishing Blow Fly Development and Sampling Procedures to Estimate Postmortem Intervals Document No. 2014.

10. Benecke, M. (2001) A brief history of forensic entomology. Forensic Science International, 120, 2-14.

11. Wheeler, W., Cartwright, P. and Hayashi, C. (1993) Arthropod Phylogeny: A combined Approach. Cladistics, 9, 1-39.

12. Block, W. (1990) Cold tolerance of insects and other arthropods. Philosophical Transactions - Royal Society of London, B, 326, 613-633. 
13. Pruna, W., Guarderas, P., Donoso, D.A. and Barragán, Á. (2019) Life cycle of Lucilia sericata (Meigen 1826) collected from Andean mountains. Neotropical Biodiversity, 5, 3-9. https://doi.org/10.1080/23766808.2019.1578056.

14. Shin, S.E., Park, J.H., Jeong, S.J. and Park, S.H. (2021) The Growth Model of Forensically Important Lucilia sericata (Meigen) (Diptera: Calliphoridae) in South Korea. Insects, 12, 323.

15. Mona, S., Jawad, M., Noreen, S., Ali, S. and Rakha, A. (2019) Forensic entomology: A comprehensive review. Advancements in Life Sciences, 6, 48-59.

16. De Sousa, J.R.P., Da Silva Carvalho-Filho, F., Esposito, M.C. and De Meyer, M. (2015) Distribution and abundance of Necrophagous Flies (Diptera: Calliphoridae and Sarcophagidae) in Maranhão, Northeastern Brazil. Journal of Insect Science, 15.

17. Moffatt, C., Heaton, V. and De Haan, D. (2016) The distribution of blow fly (Diptera: Calliphoridae) larval lengths and its implications for estimating post mortem intervals. International Journal of Legal Medicine, 130, 287-297. http://dx.doi.org/10.1007/s00414-015-1289-9.

18. Scanvion, Q., Hédouin, V. and Charabidzé, D. (2018) Collective exodigestion favours blow fly colonization and development on fresh carcasses. Animal Behaviour, 141, 221-232.

19. Aubernon, C., Hedouin, V. and Charabidze, D. (2019) The maggot, the ethologist and the forensic entomologist: Sociality and thermoregulation in necrophagous larvae. Journal of Advanced Research, 16, 67-73. https://doi.org/10.1016/j.jare.2018.12.001.

20. Casu, R.E., Eisemann, C.H., Vuocolo, T. and Tellam, R.L. (1996) The major excretory/secretory protease from Lucilia cuprina larvae is also a gut digestive protease. International Journal for Parasitology, 26, 623-628.

21. Roe, A. and Higley, L.G. (2015) Development modeling of Lucilia sericata (Diptera: Calliphoridae). PeerJ, 3, e803. https://peerj.com/articles/803.

22. Szpila, K. and Amendt, J. (2010) Current Concepts in Forensic Entomology. http://link.springer.com/10.1007/978-1-4020-9684-6.

23. Reibe, S., Doetinchem, P. V. and Madea, B. (2010) A new simulation-based model for calculating post-mortem intervals using developmental data for Lucilia sericata (Dipt.: Calliphoridae). Parasitology Research, 107, 9-16.

24. Tarone, A.M., Picard, C.J., Spiegelman, C. and Foran, D.R. (2011) Population and temperature effects on Lucilia sericata (Diptera: Calliphoridae) body size and minimum development time. Journal of Medical Entomology, 48, 1062-1068.

25. Boonsriwong, W., Sukontason, K., Olson, J.K., Vogtsberger, R.C., Chaithong, U., Kuntalue, B., et al. 
(2007) Fine structure of the alimentary canal of the larval blow fly Chrysomya megacephala (Diptera: Calliphoridae). Parasitology Research, 100, 561-574.

26. Hobson, R. (1932) Studies on the nutrition of Blow-Fly Larvae. From the Department of Entomology, London School of Hygeine and Tropical Medicine, 1932: 10.1093/oxfordjournals.aje.a118846.

27. Evans, W.A.L. (1956) Studies on the digestive enzymes of the blowfly Calliphora erythrocephala: I. The carbohydrases. Experimental Parasitology, 5, 191-206.

28. Parry, S., Linton, S.M., Francis, P.S., O’Donnell, M.J. and Toop, T. (2011) Accumulation and excretion of morphine by Calliphora stygia, an Australian blow fly species of forensic importance. Journal of Insect Physiology, 57, 62-73. http://dx.doi.org/10.1016/j.jinsphys.2010.09.005.

29. O’Donnell, M. (2008) Insect Excretory Mechanisms. In Advances in Insect Physiology, Volume 35. Advances in insect physiology, pp. 1-122. http://dx.doi.org/10.1016/S0065-2806(08)00001-5.

30. Archer, M.S., Bassed, R.B., Briggs, C.A. and Lynch, M.J. (2005) Social isolation and delayed discovery of bodies in houses: The value of forensic pathology, anthropology, odontology and entomology in the medico-legal investigation. Forensic Science International, 151, 259-265.

31. Tomberlin, J.K., Benbow, M.E., Tarone, A.M. and Mohr, R.M. (2011) Basic research in evolution and ecology enhances forensics. Trends in Ecology and Evolution, 26, 53-55.

32. Mohr, R.M. and Tomberlin, J.K. (2015) Development and validation of a new technique for estimating a minimum postmortem interval using adult blow fly (Diptera: Calliphoridae) carcass attendance. International Journal of Legal Medicine, 129, 851-859.

33. Sharma, R., Kumar Garg, R. and Gaur, J.R. (2015) Various methods for the estimation of the post mortem interval from Calliphoridae: A review. Egyptian Journal of Forensic Sciences, 5, 1-12. http://dx.doi.org/10.1016/j.ejfs.2013.04.002.

34. Acikgoz, H.N., Ilgit, E.E. and Taleb, M. (2018) Assessment of entomological remains from soil samples collected from a pig (Sus scrofa domestica) carcass decomposition site after 13 years. Turkish Journal of Parasitology, 2018: 10.5152/tpd.2018.5917. http://turkiyeparazitolderg.org/eng/makale/1097/54/Full-Text.

35. Verma, K. and Paul, R. (2016) Lucilia sericata (Meigen) and Chrysomya megacephala (Fabricius) (Diptera: Calliphoridae) Development Rate and its Implications for Forensic Entomology. Journal of Forensic Science and Medicine, 2, 146. http://www.jfsmonline.com/text.asp?2016/2/3/146/191466.

36. K Tomberlin, J. (2012) Assessment of decomposition studies indicates need for standardized and repeatable research methods in forensic entomology. Journal of Forensic Research, 03. 
37. Wang, M., Wang, Y., Hu, G., Wang, Y., Xu, W., Wu, M., et al. (2020) Development of Lucilia sericata (Diptera: Calliphoridae) under constant temperatures and its significance for the estimation of time of death. Journal of Medical Entomology, 57, 1373-1381.

38. Byrd, Jason H Peace, M.R. (2011) Entomotoxicology : Drugs, Toxins, and Insects. In Forensic Chemistry Handbook. pp. 483-500.

39. Chophi, R., Sharma, S., Sharma, S. and Singh, R. (2019) Forensic entomotoxicology: Current concepts, trends and challenges. Journal of Forensic and Legal Medicine, 67, 28-36. https://doi.org/10.1016/j.jflm.2019.07.010.

40. Peace, M. (2005) Forensic entomotoxicology: A study in the deposition and effects of amphetamines and barbiturates in the larvae of the black blow fly, Phormia regina. 2005. http://search.proquest.com/docview/305342866/.

41. Magni, P.A., Pazzi, M., Vincenti, M., Alladio, E., Brandimarte, M. and Dadour, I.R. (2016) Development and validation of a GC-MS method for nicotine detection in Calliphora vomitoria (L.) (Diptera: Calliphoridae). Forensic Science International, 261, 53-60. http://dx.doi.org/10.1016/j.forsciint.2015.11.014.

42. Sallawad, S.S., Sharma, A., Pandey, D. and Ahirwar, B. (2018) Entomotoxicology- A Juvenile Branch of Forensic Entomological Studies. Research Journal of Pharmacy and Technology, 11, 65-72.

43. Basilicata, P., Pieri, M., Simonelli, A., Faillace, D., Niola, M. and Graziano, V. (2019) Application of a chemiluminescence immunoassay system and GC/MS for toxicological investigations on skeletonized human remains. Forensic Science International, 300, 120-124. https://doi.org/10.1016/j.forsciint.2019.05.003.

44. Gosselin, M., Di Fazio, V., Wille, S.M.R., Ramírez Fernandez, M. del M., Samyn, N., Bourel, B., et al. (2011) Methadone determination in puparia and its effect on the development of Lucilia sericata (Diptera, Calliphoridae). Forensic Science International, 209, 154-159.

45. El-Samad, L.M., El-Moaty, Z.A. and Makemer, H.M. (2011) Effects of tramadol on the development of Lucilia sericata (Diptera: Calliphoridae) and detection of the drug concentration in postmortem rabbit tissues and larvae. Journal of Entomology, 8, 353-364.

46. Mullany, C., Keller, P.A., Nugraha, A.S. and Wallman, J.F. (2014) Effects of methamphetamine and its primary human metabolite, $\mathrm{p}$-hydroxymethamphetamine, on the development of the Australian blowfly Calliphora stygia. Forensic Science International, 241, 102-111.

http://dx.doi.org/10.1016/j.forsciint.2014.05.003.

47. George, K.A., Archer, M.S., Green, L.M., Conlan, X.A. and Toop, T. (2009) Effect of morphine on the 
growth rate of Calliphora stygia (Fabricius) (Diptera: Calliphoridae) and possible implications for forensic entomology. Forensic Science International, 193, 21-25.

48. De Carvalho, L.M.L., Linhares, A.X. and Badan Palhares, F.A. (2012) The effect of cocaine on the development rate of immatures and adults of Chrysomya albiceps and Chrysomya putoria (Diptera: Calliphoridae) and its importance to postmortem interval estimate. Forensic Science International, 220, 27-32.

49. Zou, Y., Huang, M., Huang, R., Wu, X., You, Z., Lin, J., et al. (2013) Effect of ketamine on the development of Lucilia sericata (Meigen) (Diptera: Calliphoridae) and preliminary pathological observation of larvae. Forensic Science International, 226, 273-281. http://dx.doi.org/10.1016/j.forsciint.2013.01.042.

50. Hodecek, J. (2020) Revisiting the concept of entomotoxicology. Forensic Science International: Synergy, 2, 282-286.

51. Verma, K. and Mp, R.P. (2013) Entomology , Ornithology \& Herpetology Assessment of Post Mortem Interval , ( PMI ) from Forensic Entomotoxicological Studies of Larvae and Flies. 1, 2-5.

52. Karampela, S., Pistos, C., Moraitis, K., Stoukas, V., Papoutsis, I., Zorba, E., et al. (2015) Development and validation of a LC/MS method for the determination of 89 -tetrahydrocannabinol and 11 carboxy- $\delta 9$-tetrahydrocannabinol in the larvae of the blowfly Lucilia sericata: Forensic applications. Science and Justice, 55, 472-480. http://dx.doi.org/10.1016/j.scijus.2015.06.003.

53. Rezende, F., Alonso, M.A., Souza, C.M., Thyssen, P.J. and Linhares, A.X. (2014) Developmental rates of immatures of three Chrysomya species (Diptera: Calliphoridae) under the effect of methylphenidate hydrochloride, phenobarbital, and methylphenidate hydrochloride associated with phenobarbital. Parasitology Research, 113, 1897-1907.

54. Fathy, H.M., Attia, R.A.H., Yones, D.A., Eldeek, H.E.M.E., Tolba, M.E.M. and Shaheen, M.S.I. (2008) Effect of Codeine Phosphate on Developmental Stages of Forensically Important Calliphoride Fly : By. Mansoura Journal of Forensic Medicine and Clinical Toxicology, XVI, 41-59.

55. Carvalho, L.M.L., Linhares, A.X. and Trigo, J.R. (2001) Determination of drug levels and the effect of diazepam on the growth of necrophagous flies of forensic importance in southeastern Brazil. Forensic Science International, 120, 140-144.

56. Kintz, P., Godelar, B., Tracqui, a, Mangin, P., Lugnier, a a and Chaumont, a J. (1990) Fly larvae: a new toxicological method of investigation in forensic medicine. Journal of forensic sciences, 35 , 204-7. http://www.ncbi.nlm.nih.gov/pubmed/2313261.

57. Campobasso, C., Gherardi, M., Caligara, M., Sironi, L. and Introna, F. (2004) Drug analysis in blowfly 
larvae and in human tissues: a comparative study. International Journal of Legal Medicine, 118, 210-214. http://link.springer.com/10.1007/s00414-004-0448-1.

58. Beyer, C., Enos, F., Stajié, M., Ph, D. and Virginia, N. (1980) Drug identification identification through through analysis analysis of maggots maggots drug. J Forensic Sci, 411, 411-412.

59. Bugelli, V., Papi, L., Fornaro, S., Stefanelli, F., Chericoni, S., Giusiani, M., et al. (2017)

Entomotoxicology in burnt bodies: a case of maternal filicide-suicide by fire. International Journal of Legal Medicine, 131, 1299-1306.

60. Rueda, L.C., Ortega, L.G., Segura, N.A., Acero, V.M. and Bello, F. (2010) Lucilia sericata strain from Colombia: Experimental colonization, life tables and evaluation of two artifcial diets of the blowfy Lucilia sericata (Meigen) (Diptera: Calliphoridae), Bogotá, Colombia strain. Biological Research, 43, 197-203.

61. da Silva, E.I.T., Wilhelmi, B. and Villet, M.H. (2017) Forensic entomotoxicology revisited-towards professional standardisation of study designs. International Journal of Legal Medicine, 131, 13991412.

62. Jones, N., Whitworth, T. and Marshall, S.A. (2019) Blow flies of North America: Keys to the subfamilies and genera of Calliphoridae, and to the species of the subfamilies Calliphorinae, Luciliinae and Chrysomyinae. Canadian Journal of Arthropod Identification, 99.

63. Skopp, G. (2010) Postmortem toxicology. Forensic Science, Medicine, and Pathology, 6, 314-325.

64. Palamalai, V., Olson, K.N., Kloss, J., Middleton, O., Mills, K., Strobl, A.Q., et al. (2013) Superiority of postmortem liver fentanyl concentrations over peripheral blood influenced by postmortem interval for determination of fentanyl toxicity. Clinical Biochemistry, 46, 598-602. http://dx.doi.org/10.1016/j.clinbiochem.2013.02.001.

65. Luckenbill, K., Thompson, J., Middleton, O., Kloss, J. and Apple, F. (2008) Fentanyl postmortem redistribution: preliminary findings regarding the relationship among femoral blood and liver and heart tissue concentrations. Journal of Analytical Toxicology, 32, 639-643.

66. Olson, K.N., Luckenbill, K., Thompson, J., Middleton, O., Geiselhart, R., Mills, K.M., et al. (2010) Postmortem redistribution of fentanyl in blood. American Journal of Clinical Pathology, 133, 447453.

67. Grebe, S.K.G. and Singh, R.J. (2011) LC-MS/MS in the clinical laboratory - where to from here? The Clinical Biochemist Reviews, 32, 5-31.

68. Othmer, D.F., Bergen, W.S., Shlechter, N. and Bruins, P.F. (1945) Liquid-Liquid Extraction Data. Industrial \& Engineering Chemistry, 37, 890-894. 
69. Rojkiewicz, M., Majchrzak, M., Celiński, R., Kuś, P. and Sajewicz, M. (2017) Identification and physicochemical characterization of 4-fluorobutyrfentanyl (1-((4-fluorophenyl)(1phenethylpiperidin-4-yl)amino)butan-1-one, 4-FBF) in seized materials and post-mortem biological samples. Drug Testing and Analysis, 9, 405-414.

70. Coopman, V., Cordonnier, J., De Leeuw, M. and Cirimele, V. (2016) Ocfentanil overdose fatality in the recreational drug scene. Forensic Science International, 266, 469-473. http://dx.doi.org/10.1016/j.forsciint.2016.07.005.

71. Andrade-Eiroa, A., Canle, M., Leroy-Cancellieri, V. and Cerdà, V. (2016) Solid-phase extraction of organic compounds: A critical review (Part I). TrAC - Trends in Analytical Chemistry, 80, 641-654. http://dx.doi.org/10.1016/j.trac.2015.08.015.

72. Haginaka, J. (2005) Selectivity of affinity media in solid-phase extraction of analytes. TrAC - Trends in Analytical Chemistry, 24, 407-415.

73. Shoff, E.N., Zaney, M.E., Kahl, J.H., Hime, G.W. and Boland, D.M. (2017) Qualitative identification of fentanyl analogs and other opioids in postmortem cases by UHPLC-Ion Trap-MSn. Journal of Analytical Toxicology, 41, 484-492.

74. Palmquist, K.B. and Swortwood, M.J. (2020) Quantification of Furanylfentanyl and its Metabolites in Human and Rat Plasma Using LC-MS-MS. Journal of analytical toxicology, 44, 589-595.

75. Huang, Z.P., Chen, X.H., Wijsbeek, J., Franke, J.P. and De Zeeuw, R.A. (1996) An enzymic digestion and solid-phase extraction procedure for the screening for acidic, neutral, and basic drugs in liver using gas chromatography for analysis. Journal of Analytical Toxicology, 20, 248-254.

76. Koesukwiwat, U., Jayanta, S. and Leepipatpiboon, N. (2007) Solid-phase extraction for multiresidue determination of sulfonamides, tetracyclines, and pyrimethamine in Bovine's milk. Journal of Chromatography A, 1149, 102-111.

77. Truver, M.T. and Swortwood, M.J. (2018) Quantitative Analysis of Novel Synthetic Opioids, Morphine and Buprenorphine in Oral Fluid by LC-MS-MS. Journal of Analytical Toxicology, 42, 554-561.

78. Jenkins, K.M., Young, M.S., Mallet, C.R. and Elian, A.A. (2004) Mixed-Mode Solid-Phase Extraction Procedures for the Determination of MDMA and Metabolites in Urine Using LC-MS, LC-UV, or GCNPD. Journal of Analytical Toxicology, 28, 50-58.

79. Anastassiades, M., Lehotay, S., Stajnbaher, D. and Schenck, F. (2003) Fast and easy multiresidue method employing acetonitrile extraction/partitioning and 'dispersive solid-phase extraction' for the determination of pesticide residues in produce. Journal of Aoac International, 86, 412-431. 80. Vudathala, D., Cummings, M. and Murphy, L. (2010) Analysis of multiple anticoagulant rodenticides 
in animal blood and liver tissue using principles of QuEChERS method. Journal of Analytical Toxicology, 34, 273-279.

81. Matsuta, S., Nakanishi, K., Miki, A., Zaitsu, K., Shima, N., Kamata, T., et al. (2013) Development of a simple one-pot extraction method for various drugs and metabolites of forensic interest in blood by modifying the QuEChERS method. Forensic Science International, 232, 40-45. http://dx.doi.org/10.1016/j.forsciint.2013.06.015.

82. Yonemitsu, K., Sasao, A., Mishima, S., Ohtsu, Y. and Nishitani, Y. (2016) A fatal poisoning case by intravenous injection of "bath salts" containing acetyl fentanyl and 4-methoxy PV8. Forensic Science International, 267, e6-e9. http://dx.doi.org/10.1016/j.forsciint.2016.08.025.

83. Pouliopoulos, A., Tsakelidou, E., Krokos, A., Gika, H.G., Theodoridis, G. and Raikos, N. (2018) Quantification of 15 Psychotropic Drugs in Serum and Postmortem Blood Samples after a Modified Mini-QuEChERS by UHPLC-MS-MS. Journal of Analytical Toxicology, 42, 337-345.

84. Hedegaard, H., Miniño, A.M. and Warner, M. (2020) Drug Overdose Deaths in the United States, 1999-2019. NCHS data brief, 2020.

85. Mattson, C.L., Tanz, L.J., Quinn, K., Kariisa, M., Patel, P. and Davis, N.L. (2021) Morbidity and Mortality Weekly Report Trends and Geographic Patterns in Drug and Synthetic Opioid Overdose Deaths-United States, 2013-2019. Morbidity and Mortality Weekly Report, 70, 202-207. https://www.cdc.gov/nchs/data/nvsr/nvsr61/.

86. Stanley, T.H. (1992) The history and development of the fentanyl series. Journal of Pain and Symptom Management, 7, 3-7.

87. Raffa, R.B., Pergolizzi, J. V., LeQuang, J.A., Taylor, R., Colucci, S. and Annabi, M.H. (2018) The fentanyl family: A distinguished medical history tainted by abuse. Journal of Clinical Pharmacy and Therapeutics, 43, 154-158.

88. van Rijn, R.M., Whistler, J.L. and Waldhoer, M. (2010) Opioid-receptor-heteromer-specific trafficking and pharmacology. Current Opinion in Pharmacology, 10, 73-79.

89. Corder, G., Castro, D.C., Bruchas, M.R. and Scherrer, G. (2018) Endogenous and Exogenous Opioids in Pain HHS Public Access. Annu Rev Neurosci, 41, 453-473.

90. Stein, C. (2016) Opioid receptors. Annual Review of Medicine, 67, 433-451.

91. Wilde, M., Pichini, S., Pacifici, R., Tagliabracci, A., Busardò, F.P., Auwärter, V., et al. (2019) Metabolic Pathways and Potencies of New Fentanyl Analogs. Frontiers in Pharmacology, 10, 1-16.

92. Tabarra, I., Soares, S., Rosado, T., Gonçalves, J., Luís, Â., Malaca, S., et al. (2019) Novel synthetic opioids-toxicological aspects and analysis. Forensic Sciences Research, 4, 111-140. 
93. Kennedy, M.C. (2010) Post-mortem drug concentrations. Internal Medicine Journal, 40, 183-187.

94. Baselt, R. (2002) Fentanyl. Disposition of toxic drugs and chemicals in man. 6th ed., Biomedical Publications, Seal Beach, CA.

95. Peng, P. and Sandler, A. (1999) A Review of the Use of Fentanyl Analgesia in the Management of Acute Pain in Adults. Anesthesiology, 90, 576-599.

96. Strayer, K.E., Antonides, H.M., Juhascik, M.P., Daniulaityte, R. and Sizemore, I.E. (2018) LC-MS/MSbased method for the multiplex detection of 24 fentanyl analogues and metabolites in whole blood at Sub ng $\mathrm{mL}-1$ concentrations. ACS Omega, 3, 514-523.

97. Fogarty, M.F., Papsun, D.M. and Logan, B.K. (2018) Analysis of Fentanyl and 18 Novel Fentanyl Analogs and Metabolites by LC-MS-MS, and report of Fatalities Associated with Methoxyacetylfentanyl and Cyclopropylfentanyl. Journal of Analytical Toxicology, 42, 592-604.

98. Jannetto, P.J., Helander, A., Garg, U., Janis, G.C., Goldberger, B. and Ketha, H. (2019) The fentanyl epidemic and evolution of fentanyl analogs in the United States and the European Union. Clinical Chemistry, 65, 242-253.

99. Prekupec, M.P., Mansky, P.A. and Baumann, M.H. (2017) Misuse of novel synthetic opioids: a deadly new trend. Journal of Addiction Medicine, 11, 256-265.

100. Lamy, F.R., Daniulaityte, R., Barratt, M.J., Lokala, U., Sheth, A. and Carlson, R.G. (2020) Listed for sale: Analyzing data on fentanyl, fentanyl analogs and other novel synthetic opioids on one cryptomarket. Drug and Alcohol Dependence, 213, 108115. https://doi.org/10.1016/j.drugalcdep.2020.108115.

101. Wharton, R.E., Casbohm, J., Hoffmaster, R., Brewer, B.N., Finn, M.G. and Johnson, R.C. (2021) Detection of 30 Fentanyl Analogs by Commercial Immunoassay Kits. Journal of analytical toxicology, 45, 111-116.

102. Labroo, R.B., Paine, M.F., Thummel, K.E. and Kharasch, E.D. (1997) Fentanyl metabolism by human hepatic and intestinal cytochrome P450 3A4: implications for individual variablity in disposition, efficacy, and drug interactions. Drug Metabolism and Disposition, 25, 1072-1080.

103. Kharbouche, H., Augsburger, M., Cherix, D., Sporkert, F., Giroud, C., Wyss, C., et al. (2008) Codeine accumulation and elimination in larvae, pupae, and imago of the blowfly Lucilia sericata and effects on its development. International Journal of Legal Medicine, 122, 205-211.

104. American Academy of Forensic Science (AAFS) Standard Board (ASB). Standard 036. Standard Practices for Method Validation in Forensic Toxicology. First Edition. (2019) American Academy of Forensic Sciences, 2019. http://www. asbstandardsboard.org/wp- 
content/uploads/2019/11/036_Std_e1.pdf (6 December 2019).

105. Lebeau, M.A. (2020) ANSI/ASB Standard 036 for Method Validation in Forensic Toxicology Has Replaced SWGTOX's Version. Journal of Analytical Toxicology, 44, 414.

106. Magalhães, E.J., Queiroz, M.E.L.R. de, Penido, M.L. de O., Paiva, M.A.R., Teodoro, J.A.R., Augusti, R., et al. (2013) Determination of cocaine in postmortem human liver exposed to overdose.

Application of an innovative and efficient extraction/clean up procedure and gas chromatographymass spectrometry analysis. Journal of Chromatography A, 1309, 15-21.

http://dx.doi.org/10.1016/j.chroma.2013.08.039.

107. Matuszewski, B.K., Constanzer, M.L. and Chavez-Eng, C.M. (2003) Strategies for the assessment of matrix effect in quantitative bioanalytical methods based on HPLC-MS/MS. Analytical Chemistry, 75, 3019-3030.

108. Tracqui, A., Keyser-Tracqui, C., Kintz, P. and Ludes, B. (2004) Entomotoxicology for the forensic toxicologist: much ado about nothing? International Journal of Legal Medicine, 118, 194-196. http://link.springer.com/10.1007/s00414-004-0442-7.

109. Williams, Keith R. B.Sc.; Pounder, Derrick J. F.R.C.P.A., M.R.C.P. (1997) Site-to-Site Variability of Drug Concentrations in Skeletal Muscle. The American Journal of Forensic Medicine and Pathology, 1997.

110. Wallace, D.R. (2017) Evolution of Forensic Entomotoxicology. TOXICOLOGY AND FORENSIC MEDICINE, Special Ed, 1-4. http://dx.doi.org/10.17140/TFMOJ-SE-1-e001.

111. Gosselin, M., Wille, S.M.R., Ramı, M., Samyn, N., Boeck, G. De, Bourel, B., et al. (2011) Entomotoxicology, experimental set-up and interpretation for forensic toxicologists. 208, 1-9.

112. Maurer, H.H. (2005) Advances in analytical toxicology: the current role of liquid chromatography mass spectrometry in drug quantification in blood and oral fluid. Analytical and Bioanalytical Chemistry, 381, 110-118.

113. O'Donnell, J.K., Halpin, J., Mattson, C.L., Goldberger, B.A. and Gladden, R.M. (2017) Deaths Involving Fentanyl, Fentanyl Analogs, and U-47700 - 10 States, July-December 2016. MMWR. Morbidity and Mortality Weekly Report, 66, 1197-1202. http://www.cdc.gov/mmwr/volumes/66/wr/mm6643e1.htm.

114. Marchei, E., Pacifici, R., Mannocchi, G., Marinelli, E., Busardò, F.P. and Pichini, S. (2018) New synthetic opioids in biological and non-biological matrices: a review of current analytical methods. Trends in Analytical Chemistry, 102, 1-15.

115. McIntyre, I.M. (2012) Postmortem fentanyl concentrations: a review. Journal of Forensic Research, 
01. https://www.omicsonline.org/2157-7145/2157-7145-3-157.digital/2157-7145-3-157.html.

116. Andresen, H., Gullans, A., Veselinovic, M., Anders, S., Schmoldt, A., Iwersen-Bergmann, S., et al. (2012) Fentanyl: toxic or therapeutic? Postmortem and antemortem blood concentrations after transdermal fentanyl application. Journal of Analytical Toxicology, 36, 182-194.

117. McIntyre, I.M. (2014) Liver and peripheral blood concentration ratio (I/p) as a marker of postmortem drug redistribution: a literature review. Forensic Science, Medicine, and Pathology, 10, 91-96.

118. Pounder, D.J. and Osselton, M.D. (2015) Autopsy findings: postmortem drug sampling and redistribution. In Encyclopedia of Forensic and Legal Medicine: Second Edition, Volume 1. Elsevier Ltd., pp. 348-353. http://dx.doi.org/10.1016/B978-0-12-800034-2.00045-8.

119. Fields, M.D., Abate, M.A., Hu, L., Long, D.L., Blommel, M.L., Haikal, N.A., et al. (2015) Parent and Metabolite Opioid Drug Concentrations in Unintentional Deaths Involving Opioid and Benzodiazepine Combinations. Journal of Forensic Sciences, 60, 950-956.

120. Concheiro, M., Chesser, R., Pardi, J. and Cooper, G. (2018) Postmortem toxicology of new synthetic opioids. Frontiers in Pharmacology, 9, 1-18.

121. Crum, E.D., Bailey, K.M., Richards-Waugh, L.L., Clay, D.J., Gebhardt, M.A. and Kraner, J.C. (2013) Validation of blood and liver oxymorphone analysis using LC-MS-MS: Concentrations in 30 fatal overdoses. Journal of Analytical Toxicology, 37, 512-516.

122. McIntyre, I.M., Trochta, A., Gary, R.D., Wright, J. and Mena, O. (2016) An acute butyr-fentanyl fatality: A case report with postmortem concentrations. Journal of Analytical Toxicology, 40, 162166.

123. Miyaguchi, H., Kakuta, M., Iwata, Y.T., Matsuda, H., Tazawa, H., Kimura, H., et al. (2007) Development of a micropulverized extraction method for rapid toxicological analysis of methamphetamine in hair. Journal of Chromatography $A, 1163,43-48$.

124. Ahern, B.J., Soma, L.R., Rudy, J.A., Uboh, C.E. and Schaer, T.P. (2010) Pharmacokinetics of fentanyl administered transdermally and intravenously in sheep. American Journal of Veterinary Research, 71, 1127-1132.

125. Boom, M., Olofsen, E., Neukirchen, M., Fussen, R., Hay, J., Jan Groeneveld, G., et al. (2013) Fentanyl Utility Function. Anesthesiology, 119, 663-674.

126. Gerace, Enrico; Salomone, Alberto; Vincenti, M. (2018) Analytical Approaches in Fatal Intoxication Cases Involving New Synthetic Opioids. CURRENT PHARMACEUTICAL BIOTECHNOLOGY, 19, 113123. 
127. Larabi, I.A., Martin, M., Fabresse, N., Etting, I., Edel, Y., Pfau, G., et al. (2020) Hair testing for 3fluorofentanyl, furanylfentanyl, methoxyacetylfentanyl, carfentanil, acetylfentanyl and fentanyl by LC-MS/MS after unintentional overdose. Forensic Toxicology, 38, 277-286. https://doi.org/10.1007/s11419-019-00502-0.

128. Elliott, S.P. and Hernandez Lopez, E. (2018) A Series of Deaths Involving Carfentanil in the UK and Associated Post-mortem Blood Concentrations. Journal of analytical toxicology, 42, e41-e45.

129. Seither, J. and Reidy, L. (2017) Confirmation of carfentanil, U-47700 and other synthetic opioids in a human performance case by LC-MS-MS. Journal of Analytical Toxicology, 41, 493-497.

130. Papsun, D., Isenschmid, D. and Logan, B.K. (2017) Observed carfentanil concentrations in 355 blood specimens from forensic investigations. Journal of Analytical Toxicology, 41, 777-778.

131. Schuchat, A., Houry, D. and Guy, G.P. (2017) New data on opiod use and prescribing in the United States. Jama, 318, 425-426.

132. Wang, L. and Bernert, J.T. (2006) Analysis of 13 fentanils, including sufentanil and carfentanil, in human urine by liquid chromatography-atmospheric-pressure ionization-tandem mass spectrometry. Journal of Analytical Toxicology, 30, 335-341.

133. Poklis, J., Poklis, A., Wolf, C., Mainland, M., Hair, L., Devers, K., et al. (2015) Postmortem tissue distribution of acetyl fentanyl, fentanyl and their respective nor-metabolites analyzed by ultrahigh performance liquid chromatography with tandem mass spectrometry. Forensic Science International, 257, 435-441.

134. Yarema, M.C.M.. and Becker, C.E.M.D. (2005) Key Concepts in Postmortem Drug Redistribution. Clinical toxicology, 43, 235-41.

135. Chesser, R., Pardi, J., Concheiro, M. and Cooper, G. (2019) Distribution of synthetic opioids in postmortem blood, vitreous humor and brain. Forensic Science International, 305, 109999. https://doi.org/10.1016/j.forsciint.2019.109999.

136. Ioan, B.G., Manea, C., Hanganu, B., Statescu, L., Solovastru, L.G. and Manoilescu, I. (2017) The chemistry decomposition in human corpses. Revista de Chimie, 68, 1450-1454.

137. Sijens, P.E., Edens, M.A., Bakker, S.J.L. and Stolk, R.P. (2010) MRI-determined fat content of human liver, pancreas and kidney. World Journal of Gastroenterology, 16, 1993-1998.

138. Kotronen, A., Seppänen-Laakso, T., Westerbacka, J., Kiviluoto, T., Arola, J., Ruskeepää, A.L., et al. (2010) Comparison of lipid and fatty acid composition of the liver, subcutaneous and intraabdominal adipose tissue, and serum. Obesity, 18, 937-944. http://dx.doi.org/10.1038/oby.2009.326. 
139. Daniulaityte, R., Juhascik, M.P., Strayer, K.E., Sizemore, I.E., Zatreh, M., Nahhas, R.W., et al. (2019) Trends in fentanyl and fentanyl analogue-related overdose deaths - Montgomery County, Ohio, 2015-2017. Drug and Alcohol Dependence, 198, 116-120. https://doi.org/10.1016/j.drugalcdep.2019.01.045.

140. Seymour, C., Shaner, R.L., Feyereisen, M.C., Wharton, R.E., Kaplan, P., Hamelin, E.I., et al. (2019) Determination of fentanyl analog exposure using dried blood spots with LC-MS-MS. Journal of Analytical Toxicology, 43, 266-276.

141. Sofalvi, S., Schueler, H.E., Lavins, E.S., Kaspar, C.K., Brooker, I.T., Mazzola, C.D., et al. (2017) An LCMS-MS method for the analysis of carfentanil, 3-methylfentanyl, 2-furanyl fentanyl, acetyl fentanyl, fentanyl and norfentanyl in postmortem and impaired-driving cases. Journal of Analytical Toxicology, 41, 473-483.

142. Kahl, J.H., Gonyea, J., Humphrey, S.M., Hime, G.W. and Boland, D.M. (2018) Quantitative Analysis of Fentanyl and Six Fentanyl Analogs in Postmortem Specimens by UHPLC-MS-MS. Journal of Analytical Toxicology, 42, 570-580.

143. Seth, P., Rudd, R.A., Noonan, R.K. and Haegerich, T.M. (2018) Quantifying the Epidemic of Prescription Opioid Overdose Deaths. American Journal of Public Health, 108, 500-502. http://ajph.aphapublications.org/doi/10.2105/AJPH.2017.304265.

144. Ishak, N., Ahmad, A.H., Mohamad Noor, S.A. and Ahmad, A. (2019) Detection of heroin metabolites at different developmental stages of Lucilia cuprina (Diptera: Calliphoridae) reared in herointreated meat: a preliminary analysis. Egyptian Journal of Forensic Sciences, 9, 1-9.

145. Kinnear, B.J.F., Martin, M., Thomson, J.A. and Neufeld, G.J. (1968) Developmental Changes In The Late Larva Of Calliphora Stygia. Australian Journal of Biological Sciences, 1968.

146. Stubbings, G. and Bigwood, T. (2009) The development and validation of a multiclass liquid chromatography tandem mass spectrometry (LC-MS/MS) procedure for the determination of veterinary drug residues in animal tissue using a QuEChERS (QUick, Easy, CHeap, Effective, Rugged and Safe) approac. Analytica Chimica Acta, 637, 68-78.

147. Amorim Alves, E., Agonia, A.S., Cravo, S.M., Afonso, C.M., Duarte, A., Netto, P., et al. (2017) GC-MS method for the analysis of thirteen opioids, cocaine and cocaethylene in whole blood based on a modified Quechers extraction. Current Pharmaceutical Analysis, 13, 215-223.

148. Tartaglia, A., D’Ambrosio, F., Ramundo, P., Ferrone, V., Ricci, D. and Locatelli, M. (2020) Innovative approach to increase sensibility and selectivity in analytical chemistry: QuEChERS method. Reviews in Separation Sciences, 2, 19-34. 
149. Bugelli, V., Campobasso, C. Pietro, Verhoff, M.A. and Amendt, J. (2017) Effects of different storage and measuring methods on larval length values for the blow flies (Diptera: Calliphoridae) Lucilia sericata and Calliphora vicina. Science and Justice, 57, 159-164.

http://dx.doi.org/10.1016/j.scijus.2016.10.008.

150. Kotze, A.C. (1993) Cytochrome-p450 monooxygenases in larvae of insecticide-susceptible and insecticide-resistant strains of the australian sheep blowfly Lucilia-cuprina. Pesticide Biochemistry and Physiology, 46, 65-72.

151. Kotze, A.C. and Sales, N. (2001) Inheritance of diflubenzuron resistance and monooxygenase activities in a laboratory-selected strain of Lucilia cuprina (Diptera: Calliphoridae). Journal of Economic Entomology, 94, 1243-1248.

152. Wilson, J.A., Haack, N.A., Heath, A.C.G. and Clark, A.G. (2001) Resistance to diazinon in New Zealand populations of the European green blowfly Lucilia sericata. GEN. APPL. ENT., 30, 55-62.

153. Traylor MJ, Baek JM, Richards KE, Fusetto R, H. (2017) Recombinant expression and characterization of Lucilia cuprina CYP6G3: Activity and binding properties toward multiple pesticides. Insect biochemistry and molecular biology, 90, 14-22.

154. Bourel, B., Fleurisse, L., Hédouin, V., Cailliez, J.C., Creusy, C., Gosset, D., et al. (2001) Immunohistochemical contribution to the study of morphine metabolism in Calliphoridae larvae and implications in forensic entomotoxicology. Journal of forensic sciences, 46, 596-9. http://www.ncbi.nlm.nih.gov/pubmed/11372995.

155. Davidowitz, G. and Nijhout, H.F. (2004) The physiological basis of reaction norms: The interaction among growth rate, the duration of growth and body size. Integrative and Comparative Biology, 44, 443-449.

156. Grassberger, M. and Reiter, C. (2001) Effect of temperature on Lucilia sericata (Diptera: Calliphoridae) development with special reference to the isomegalen- and isomorphen-diagram. Forensic Science International, 120, 32-36.

157. Bhardwaj, T., Sharma, S., Dalal, J. and Verma, \& K. (2020) The implication of morphometrics and growth rate of dipteran flies in forensic entomotoxicology research: a review. The Science of Nature, 107, 50.

158. Goff, M.L., Brown, W.A., Hewadikaram, K.A. and Omori, A.I. (1991) Effect of heroin in decomposing tissues on the development rate of Boettcherisca peregrina (Diptera, Sarcophagidae) and implications of this effect on estimation of postmortem intervals using arthropod development patterns. Journal of forensic sciences, 36, 537-42. http://europepmc.org/abstract/MED/2066728. 
159. Bourel, B., Hédouin, V., Martin-Bouyer, L., Bécart, A., Tournel, G., Deveaux, M., et al. (1999) Effects of morphine in decomposing bodies on the development of Lucilia sericata (Diptera: Calliphoridae). Journal of forensic sciences, 44, 354-8. http://www.ncbi.nlm.nih.gov/pubmed/10097362.

160. Salimi, M., Rassi, Y., Ahmadi, B., Chatrabgoun, O., Jamshidi, R. and Rafizadeh, S. (2018) Effects of morphine on the biomass and development rate of Chrysomya albiceps (Diptera: Calliphoridae), a forensically important species. Tropical Biomedicine, 35, 560-570.

161. Wood, M., Laloup, M., Pien, K., Samyn, N., Morris, M., Maes, R.A.A., et al. (2003) Development of a rapid and sensitive method for the quantitation of benzodiazepines in Calliphora vicina larvae and puparia by LC-MS-MS. Journal of analytical toxicology, 27, 505-12. http://www.ncbi.nlm.nih.gov/pubmed/14607007.

162. Pien, K., Laloup, M., Pipeleers-Marichal, M., Grootaert, P., De Boeck, G., Samyn, N., et al. (2004) Toxicological data and growth characteristics of single post-feeding larvae and puparia of Calliphora vicina (Diptera: Calliphoridae) obtained from a controlled nordiazepam study. International Journal of Legal Medicine, 118, 190-193. http://link.springer.com/10.1007/s00414004-0441-8.

163. Miller, M.L. et al (1994) Isolation of Amitriptyline and Nortriptyline from Fly Puparia (Phoridae) and Beetle Exuvia (Dermestidae) Associated with Mummified Human Remains. Journal of Forensic Sciences, 39, 1305-1313.

164. Maddrell, S.H. and Gardiner, B.O. (1976) Excretion of alkaloids by malpighian tubules of insects. Journal of experimental biology, 64, 267-281. http://jeb.biologists.org/content/64/2/267.2.full.pdf.

165. Malejko, J., Deoniziak, K., Tomczuk, M., Długokencka, J. and Godlewska-Żyłkiewicz, B. (2020) Puparial Cases as Toxicological Indicators: Bioaccumulation of Cadmium and Thallium in the Forensically Important Blowfly Lucilia sericata. Frontiers in Chemistry, 8, 1-14.

166. Braga, M.V., Pinto, Z.T., Queiroz, M.M. de C., Matsumoto, N. and Blomquist, G.J. (2013) Cuticular hydrocarbons as a tool for the identification of insect species: Puparial cases from Sarcophagidae. Acta Tropica, 228, 479-485. https://www.ncbi.nlm.nih.gov/pmc/articles/PMC3624763/pdf/nihms412728.pdf.

167. Macri, A.M., Lam, S., Powers, R.H. and Marsico, A.L.M. (2020) Differentiation of Morphologically Similar Human Head Hairs from Two Demographically Similar Individuals Using Amino Acid Ratios,. Journal of Forensic Sciences, 65, 1745-1751.

168. American Academy of Forensic Science (AAFS) Standard Board (ASB) (2019) Standard Practices for 
Method Validation in Forensic Toxicology. American Academy of Forensic Sciences, 2019.

http://www.asbstandardsboard.org/wp-content/uploads/2019/11/036_Std_e1.pdf.

169. Ireland, S. and Turner, B. (2006) The effects of larval crowding and food type on the size and development of the blowfly, Calliphora vomitoria. Forensic Science International, 159, 175-181.

170. Clark, K., Evans, L. and Wall, R. (2006) Growth rates of the blowfly, Lucilia sericata, on different body tissues. Forensic Science International, 156, 145-149.

171. Bourel, B., Tournel, G., Hedouin, V., Deveaux, M., Goff, M.L. and Gosset, D. (2001) Morphine extraction in necrophagous insects remains for determining ante-mortem opiate intoxication. Forensic Science International, 120, 127-131.

172. Rivers, D.B. and Dahlem, G.A. (2014) The Science of Forensic Entomology. John Wiley \& Sons, Ltd. 


\section{Appendix A}

\section{Joseph cox}

1330 SW 104TH PATH APT 212, MIAMI FL 33174| 512-690-3135 | jcocwvu2021@gmail.com

\section{EDUCATION}

West Virginia University

2017-Present

PhD in Forensic Science

Focus: Forensic Toxicology

Dissertation Defense November 2021

Expected Graduation December 2021

Sam Houston State University

M.S. in Forensic Science

Capstone "Preservation of Forensically Relevant Insects without Refrigeration"

Sam Houston State University

B.S. in Forensic Chemistry

2010

WORK EXPERIENCE

West Virginia University

Research Assistant

August 2017- Present

- Directly responsible for the NIJ project: Extraction and quantitation of fentanyl and metabolites from complex biological matrices to support medicolegal death investigations

- Lead researcher for the Forensic Toxicology section of the Dr. Arroyo research group

- Trained personnel and oversees toxicology projects

- Responsible for instrument maintenance, inventory, training, and quality control

ExperTox

Lab Manager

July 2016- July 2017

- Developed, standardized, evaluated procedures and techniques for tests used in the analysis of specimens

- Recruited, hired, trained, and supervised lab technicians, lab analysts and other lab personnel

- Lead troubleshooting and root cause analysis

- Responsible for proficiency testing

- In charge of blood and hair drug testing 
- Directly supervised lab analysts and certifying scientists

- Responsible for development, implementation, and evaluation for all activities for the laboratory

- Lead scientist for research and development of new methods

- Instrument maintenance: ICP-MS, LC-MS/MS, GC-MS, EIA, and ELISA

Forensic Toxicologist

June 2014- November 2015

- Lead analyst and certifying scientist for esoteric testing, unknown's analysis, and heavy metal testing

- Instrumentation: ICP-MS, LC-MS/MS, GC-MS, EIA, ELISA, and headspace GC-MS

Harris County Institute of Forensic Sciences, Houston, TX

Intern

May 2013 - August

2013

- Developed a method to determine GHB in blood and urine using gas chromatography/tandem mass

- Compiled validation statistics, performed multiple extractions, loaded multiple runs, and gained knowledge of Mass Hunter Software

- Daily lab tasks included GC calibration, daily doctor's meetings, sample logging and data processing

- Presented research at 2014 AAFS meeting.

Sam Houston State University, Huntsville, TX

Intramural Supervisor

2014

- Lead team of intramural referees and game managers

- Organized and supervised daily activity of all sports offered

- Senior official for all sports

- CPR certified and responsible for safety of staff and participants

\section{PuBlications}

\section{First Author}

1. Journal of Analytical Toxicology

February 2021

"Quantitation and Validation of 34 Fentanyl Analogs from Liver Tissue Using a QuEChERS Extraction and LC-MS-MS Analysis" https://doi.org/10.1093/jat/bkab009

Joseph Cox, Kylea Mathison, Colby Ott, Joseph DelTondo, James Kraner Anthony P Decaprio, and Luis E. ArroyoMora

2. Journal of Analytical Toxicology

February 2020

"Quantitation of Fentanyl and Metabolites from Liver Tissue Using a Validated QuEChERS Extraction and LC-MSMS Analysis" https://doi.org/10.1093/jat/bkaa006

Joseph Cox, Alex Train, Avery Field, Colby Ott, Joseph DelTondo, James Kraner, Kristen Bailey, Myron Gebhardt, and Luis E. Arroyo-Mora 


\section{Contributing Author}

1. Journal of Cannabis Research

July 2021

"Study of $\triangle 9$-tetrahydrocannabinol (THC) and cannabidiol (CBD) extraction FROM dried oral fluid spots (DOFS) and LC-MS/MS detection" https://doi.org/10.1186/s42238-021-00088-8

Roberta Gorziza, Joseph Cox, Renata Pereira Limberger, Luis E. Arroyo-Mora

\section{Journal of Electroanalytical Chemistry}

September 2020

"Electrochemical detection of fentanyl with screen-printed carbon electrodes using square-wave adsorptive stripping voltammetry for forensic applications" https://doi.org/10.1016/j.jelechem.2020.114425

Colby E.Ott, Hugo Cunha-Silva, Sara L. Kuberski, Joseph Cox, M. Julia Arcos-Martínez, Luis E. Arroyo-Mora

\section{Forensic Chemistry}

June 2020

"Extraction of dried oral fluid spots (DOFS) for the identification of drugs of abuse using liquid chromatography tandem mass spectrometry (LC-MS/MS)" https://doi.org/10.1016/i.forc.2020.100254

RobertaGorziza, Joseph Cox, Renata Pereira Limberger, Luis E. Arroyo-Mora

\section{Forensic Chemistry}

June 2020

"Development, validation and evaluation of a quantitative method for the analysis of twenty-four new psychoactive substances in oral fluid by LC-MS/MS" https://doi.org/10.1016/i.forc.2020.100231

Travon Cooman, Heloa Santos, Joseph Cox, João Francisco Allochio Filho, Keyller Bastos Borges, Wanderson Romão, Luis E. Arroyo-Mora

\section{The American Journal on Addictions}

November 2015

"Altered methamphetamine place conditioning in mice vaccinated with a succinyl-methamphetamine-tetanustoxoid vaccine"

Colin N. Haile MD, PhD Therese A. Kosten PhD Xiaoyun Y. Shen PhD Patrick W. O'Malley MD Kevin J. Winoske BA Berma M. Kinsey PhD Yan Wu MD Zhen Huang Ernest D. Lykissa PhD Naga Naidu PhD Joseph A. Cox MS Reetakshi Arora PhD Thomas R. Kosten MD Frank M. Orson MD

\section{PRESENTATIONS}

\section{SOFT Platform Presentation}

Presentation at 51st Annual Scientific Meeting of the Society of Forensic Toxicology (SOFT) meeting in Nashville, Tn "Effects of Fentanyl on Blow Fly Development and Quantitation of Fentanyl from Insect Tissue via QuEChERS Extraction and LC-MS/MS Analysis"

Joseph Cox, Katherine Davis, Kylea Mathison, Rachel Mohr, Joseph DelTondo, Luis E. Arroyo-Mora 
Presentation at 51st Annual Scientific Meeting of the Society of Forensic Toxicology (SOFT) meeting in Nashville, Tn "QuEChERS Extraction and LC-MS/MS Quantitation of Fentanyl Analogs and Metabolites from Liver Tissue to Support Medicolegal Death Investigations"

Joseph Cox, Kylea Mathison, Joseph DelTondo, James Kraner, and Luis Arroyo-Mora

\section{Crossing Forensic Borders (CFB) Webinar \\ January 2021}

Webinar hosted by WVU but organized by Amsterdam Center for Forensic Science and Medicine (CLHC)

"Extraction and quantitative analysis of fentanyl analogs and metabolites from complex biological specimens"

Joseph Cox, Kylea Mathison, Colby Ott, Joseph DelTondo, James Kraner, Anthony P. DeCaprio, Luis E Arroyo-Mora

\section{First WVU Online Forensic Graduate Symposium}

May 2020

Shaping Forensic Science: Emerging Trends in Research in Morgantown, WV

"Extraction and Quantitation of Fentanyl Analogs and Metabolites from Complex Biological Matrices to support Medicolegal Death Investigations"

Joseph Cox, Kylea Mathison, James Kraner and Luis E. Arroyo

\section{4th Annual Spring Undergraduate Research Symposium (Online)}

April 2020

Presentation at 2019 4th Annual Spring Undergraduate Research Symposium in Morgantown, WV

"LC-MS/MS analysis of 30+ fentanyl analogs in human liver to support medicolegal death investigations"

Kylea Mathison, Joseph Cox, Colby Ott, Luis E. Arroyo

\section{AAFS Platform Presentation}

Presented at 71st Annual Scientific Meeting of the American Academy of Forensic Science (AAFS) in Anaheim, Ca

“Evaluation of Postmortem Liver Samples Using a Validated QuEChERS Extraction for Fentanyl and Metabolites with LC-MS/MS Analysis"

Joseph Cox, Colby Ott, Joseph DelTondo, James Kraner, Kristen Bailey, Myron Gebhardt, and Luis Arroyo

\section{AAFS Poster Presentation

Presented at 71st Annual Scientific Meeting of the American Academy of Forensic Science (AAFS) in Anaheim, Ca

“Comprehensive Analysis of 34 Fentanyl Analogs including Carfentanil from Liver Tissue Using

QuEChERS and LC-MS/MS Analysis" 
Kylea Mathison, Joseph Cox, Colby Ott, Joseph DelTondo, James Kraner, Kristen Bailey, Myron

Gebhardt, and Luis Arroyo

\section{SOFT Poster Presentation}

October 2019

Presented research projects at 2019 Society of Forensic Toxicology (SOFT) meeting in Minneapolis, Minnesota

“Evaluation of Sample Preparation Techniques Using Hot Surface Desolvation (HSID) Interface for LC-MS/MS for Fentanyl Analogs in Pupa Tissue" Booth Poster

Joseph Cox, Colby E. Ott, Jenna Gardner, Yiling Ke, Sabra Botch-Jones, Frank Kero, Luis Arroyo

\section{11th Annual Summer Undergraduate Research Symposium}

July 2019

Presentation at 2019 11th Annual Summer Undergraduate Research Symposium in Morgantown, WV

"Forensic Analysis of Gunshot Residues Using Analytical Electrochemistry and Gas Chromatography-Mass Spectrometry"

Quaterio A. Richardson, Colby E. Ott, Courtney Vander Pyl, Joseph A. Cox, Tatiana Trejos and Luis E. Arroyo

\section{MAAFS Platform Presentation}

\section{May 2019}

Presentation at 2019 Mid-Atlantic Association of Forensic Scientists (MAAFS) Annual Meeting in Morgantown, WV

"LC-MS/MS Quantitation of Fentanyl and Metabolites from Lucilia Sericata Larvae and Liver Tissue Using a Validated Modified QuEChERS Extraction"

Joseph Cox, Colby Ott, Avery Field, and Luis Arroyo

"Forensic Electrochemistry and Mass Spectrometry: The Perfect Match to Tackle Fentanyl and other Novel Psychoactive Substances"

Colby Ott, Hugo Cunha-Silva, Joseph Cox, M. Julia Arcos-Martínez, Luis Arroyo

\section{AAFS Poster Presentation}

February 2019

Presented at 71st Annual Scientific Meeting of the American Academy of Forensic Science (AAFS) in Baltimore, MD

"Quantitation of Fentanyl and Metabolites from Lucilia Sericata Larvae and Liver Tissue Using a Modified Quick, Easy, Cheap, Effective, Rugged, and Safe (QuEChERS) Extraction with Analysis by Liquid Chromatography/Tandem Mass Spectrometry (LC/MS/MS)"

Joseph Cox, Colby Ott, and Luis E. Arroyo

\section{AAFS Poster Presentation}


"Electrochemical Tools for the Rapid Detection of Opioids and Novel Psychoactive Substances (NPS) With Confirmatory Analysis by Liquid Chromatography/Triple Quadrupole/Mass Spectrometry (LC/QqQ/MS)"

Colby Ott, Hugo Cunha-Silva, Joseph Cox, Julia Arcos-Martinez, Luis E. Arroyo-Mora

13. Undergraduate Research Day at the Capitol (URDC)

February 2019

Presentation at 2019 URDC (Undergraduate Research Day at the Capitol) in Charleston, WV

"Evaluation of Extraction Techniques for Fentanyl and Metabolites in Liver Tissue for Forensic Purposes"

Avery J. Fields, Joseph Cox, Luis E Arroyo

14. SOFT Poster Presentation

October 2018

Presented research projects at 2018 Society of Forensic Toxicology (SOFT) meeting in Minneapolis, Minnesota

"Comparison of Solid Phase Extraction and a Modified QuEChERS Method For Quantifying Fentanyl in Liver Tissue"

Joseph Cox, Alex Train, Heloa Santos, Colby Ott and Luis E. Arroyo-Mora

"DART-MS/MS and LC-MS/MS Comparison for the Analysis of Stimulants in 108 Seized Dietary Supplements"

Maira Kerpel Dos Santos, Joseph Cox, Heloa Santos, Emily Gleco, J. Tyler Davidson, Glen P. Jackson, Elsa Hnuch, Renata Pereira Limberger and Luis E. Arroyo-Mora

\section{66th ASMS Conference on Mass Spectrometry and Allied Topics}

June 2018

Coauthor for ASMS poster for the Conference on Mass Spectrometry and Allied Topics in San Diego, Ca

"Potential applications to new psychoactive substances identification in oral fluid and Damiana leaf (Turnera diffusa) by DART-MS/MS and LC-MS/MS"

Heloa Santos; Tyler J. Davidson; Joseph Cox; Glen P. Jackson; Wanderson Romao; Luis E. Arroyo

\section{MAFS Poster Presentation}

September 2017

Presented research project at 2017 MAFS meeting

"Screening and Confirmation of Stimulants Drugs in Seized Dietary Supplements by DART-MS and Liquid Chromatography Triple Quadrupole Mass Spectrometry (LC-QQQ-MS)"

Maíra Kerpel dos Santos, Emily Gleco, Joseph Cox, Glen P. Jackson, Renata Pereira Limberger1, and Luis E. Arroyo

\section{AAFS Poster Presentation}

February 2016

Presented research project at 2016 AAFS meeting

"Determination of Synthetic Hallucinogens: 25I-, 25C-, and 25B-NBOMe by LC/MS/MS using D3-25I-NBOMe Internal Standard" 
Presented research projects at 2015 SOFT meeting

"Quantitative Analysis of Synthetic Hallucinogens: 25I-Nbome, 25C-Nbome, and 25B-Nbome in Blood and Urine by LC-MS/MS"

"Quantitative Determination of Oxytocin in Blood and Pharmaceuticals by LCMSMS"

19. AAFS Poster Presentation

February 2014

Presented summer research project at 2014 AAFS meeting

“Determination of GHB in Blood and Urine Using GC-MS/MS without Derivatization”

\section{TEACHING EXPERIENCE}

\section{West Virginia University}

Teaching Assistant- to Luis Arroyo in Toxicology and Seized Drug Analysis

Responsible for creating and employing lab procedures, creation of GTA student handbook and lab protocols, and instructed students on toxicology and seized drug analysis techniques including MassHunter software, UV-VIS instrumentation, LC-MS/MS (Agilent 6470), GC/MS, and coulometric testing.

\section{West Virginia University}

Teaching Assistant- to Professor O'Brien in Crime Scene Investigation I and II

Administered tests and quizzes, was responsible for lab procedures, creation of graduate student handbook, and instructed students on CSI techniques.

\section{Sam Houston State University}

Teaching Assistant- to Professor Haines in Introduction to Organic and Biochemistry

Fall 2012

Administered tests and quizzes, was responsible for lab safety procedures, and instructed students on proper lab techniques.

\section{ORgANIZATIONS}

\section{American Academy of Forensic Sciences}

Full Member

2013- present

Full Member in the Toxicology Section

Presented at 2014, 2016, 2019, and 2020 meetings

\section{Society of Forensic Toxicologists}

Presented at the 2015, 2018, and 2019 conferences 
AWARDS

National Institute of Justice's (NIJ) Graduate Research Fellowship

2019-2021

in Science, Technology, Engineering, and Mathematics

Project entitled: Extraction and quantitation of fentanyl and metabolites from complex biological matrices to support medicolegal death investigations

Award number: 2019-R2-CX-0037

CERTIFICATIONS

National Registry of Certified Chemists

2017-2023

Toxicology Chemist 